\title{
Role of Interference and Computational Complexity in Modern Wireless Networks: Analysis, Optimization, and Design
}

\author{
Salvatore Talarico
}

Follow this and additional works at: https://researchrepository.wvu.edu/etd

\section{Recommended Citation}

Talarico, Salvatore, "Role of Interference and Computational Complexity in Modern Wireless Networks: Analysis, Optimization, and Design" (2015). Graduate Theses, Dissertations, and Problem Reports. 6768. https://researchrepository.wvu.edu/etd/6768

This Dissertation is protected by copyright and/or related rights. It has been brought to you by the The Research Repository @ WVU with permission from the rights-holder(s). You are free to use this Dissertation in any way that is permitted by the copyright and related rights legislation that applies to your use. For other uses you must obtain permission from the rights-holder(s) directly, unless additional rights are indicated by a Creative Commons license in the record and/ or on the work itself. This Dissertation has been accepted for inclusion in WVU Graduate Theses, Dissertations, and Problem Reports collection by an authorized administrator of The Research Repository @ WVU. For more information, please contact researchrepository@mail.wvu.edu. 


\title{
Role of Interference and Computational Complexity in Modern Wireless Networks: Analysis, Optimization, and Design
}

\author{
Salvatore Talarico \\ Dissertation submitted to the \\ Statler College of Engineering and Mineral Resources \\ at West Virginia University \\ in partial fulfillment of the requirements \\ for the degree of \\ Doctor of Philosophy \\ in \\ Electrical Engineering \\ Matthew C. Valenti, Ph.D., Chair \\ Brian D. Woerner, Ph.D. \\ Natalia A. Schmid, Ph.D. \\ Daryl S. Reynolds, Ph.D. \\ Marcello R. Napolitano, Ph.D.
}

Lane Department of Computer Science and Electrical Engineering

\section{Morgantown, West Virginia}

2015

Keywords: Information Theory, Interference, Spatial Modeling, Cellular Network, Optimization, Cooperative Communication, Cloud-RAN, Computational Complexity 


\author{
Abstract \\ Analysis, Optimization, and Design \\ by \\ Salvatore Talarico \\ Doctor of Philosophy in Electrical Engineering \\ West Virginia University \\ Matthew C. Valenti, Ph.D., Chair
}

Role of Interference and Computational Complexity in Modern Wireless Networks:

Owing to the popularity of smartphones, the recent widespread adoption of wireless broadband has resulted in a tremendous growth in the volume of mobile data traffic, and this growth is projected to continue unabated. In order to meet the needs of future systems, several novel technologies have been proposed, including cooperative communications, cloud radio access networks (RANs) and very densely deployed small-cell networks. For these novel networks, both interference and the limited availability of computational resources play a very important role. Therefore, the accurate modeling and analysis of interference and computation is essential to the understanding of these networks, and an enabler for more efficient design.

This dissertation focuses on four aspects of modern wireless networks: (1) Modeling and analysis of interference in single-hop wireless networks, (2) Characterizing the tradeoffs between the communication performance of wireless transmission and the computational load on the systems used to process such transmissions, (3) The optimization of wireless multiple-access networks when using cost functions that are based on the analytical findings in this dissertation, and (4) The analysis and optimization of multi-hop networks, which may optionally employ forms of cooperative communication.

The study of interference in single-hop wireless networks proceeds by assuming that the random locations of the interferers are drawn from a point process and possibly constrained to a finite area. Both the information-bearing and interfering signals propagate over channels that are subject to path loss, shadowing, and fading. A flexible model for fading, based on the Nakagami distribution, is used, though specific examples are provided for Rayleigh fading. The analysis is broken down into multiple steps, involving subsequent averaging of the performance metrics over the fading, the shadowing, and the location of the interferers with the aim to distinguish the effect of these mechanisms that operate over different time scales. The analysis is extended to accommodate diversity reception, which is important for the understanding of cooperative systems that combine transmissions that originate from different locations. Furthermore, the role of spatial correlation is considered, which provides insight into how the performance in one location is related to the performance in another location.

While it is now generally understood how to communicate close to the fundamental limits implied by information theory, operating close to the fundamental performance bounds 
is costly in terms of the computational complexity required to receive the signal. This dissertation provides a framework for understanding the tradeoffs between communication performance and the imposed complexity based on how close a system operates to the performance bounds, and it allows to accurately estimate the required data processing resources of a network under a given performance constraint. The framework is applied to Cloud-RAN, which is a new cellular architecture that moves the bulk of the signal processing away from the base stations (BSs) and towards a centralized computing cloud. The analysis developed in this part of the dissertation helps to illuminate the benefits of pooling computing assets when decoding multiple uplink signals in the cloud. Building upon these results, new approaches for wireless resource allocation are proposed, which unlike previous approaches, are aware of the computing limitations of the network.

By leveraging the accurate expressions that characterize performance in the presence of interference and fading, a methodology is described for optimizing wireless multiple-access networks. The focus is on frequency hopping $(\mathrm{FH})$ systems, which are already widely used in military systems, and are becoming more common in commercial systems. The optimization determines the best combination of modulation parameters (such as the modulation index for continuous-phase frequency-shift keying), number of hopping channels, and code rate. In addition, it accounts for the adjacent-channel interference (ACI) and determines how much of the signal spectrum should lie within the operating band of each channel, and how much can be allowed to splatter into adjacent channels.

The last part of this dissertation contemplates networks that involve multi-hop communications. Building on the analytical framework developed in early parts of this dissertation, the performance of such networks is analyzed in the presence of interference and fading, and it is introduced a novel paradigm for a rapid performance assessment of routing protocols. Such networks may involve cooperative communications, and the particular cooperative protocol studied here allows the same packet to be transmitted simultaneously by multiple transmitters and diversity combined at the receiver. The dynamics of how the cooperative protocol evolves over time is described through an absorbing Markov chain, and the analysis is able to efficiently capture the interference that arises as packets are periodically injected into the network by a common source, the temporal correlation among these packets and their interdependence. 


\section{Acknowledgements}

I would like to thank all the people who contributed in some way to the work described in this dissertation. First of all, I would like to gratefully and sincerely thank my advisor and committee chair Dr. Matthew C. Valenti for welcoming me in his group, for his guidance, understanding, and patience during my "journey" at West Virginia University (WVU). His attention to details, enthusiastic attitude, and his hard work have inspired me from the very first moment I become one of his students, and I feel blessed I have had the opportunity to work for him. He has been a terrific and supportive mentor, and has made available to me many opportunities for both my professional and personal growth, for which I will always be grateful.

I would like to thank Dr. Natalia A. Schmid, Dr. Daryl S. Reynolds, Dr. Brian D. Woerner and Dr. Marcello R. Napolitano for being in my committee, and for all their comments, questions, and feedback, that refined this work. I have been very blessed to take classes and work for some of them, and I have tremendously benefit from their knowledge and experience.

This work would have never been possible without the support of the external collaborators. I would like to thank in primis Dr. Don Torrieri, from the Army Research Lab (ARL), for giving me the opportunity to work for him and for his invaluable help for the research presented in Chaps. 2-4 and Chaps. 7-8. I would like to thank Dr. Peter Rost and Dr. Andreas Maeder from Nokia Networks for their help in Chaps. 5-6 and Dr. Thomas R. Halford from WPL Inc. for his support in Chap. 9.

I would like to thank all the students of the wireless communication research lab (WCRL) for making this journey more pleasant. A special thank goes to Terry Ferrett for inducting me to the usage of the cluster computing resources available for the WCRL'eers, which has been very useful and has speeded up considerably the completion of this work. Another thank goes to Marwan Alkhweldi, for his friendship, and his unconditioned support in all 
the difficult times.

I also wanted to thank WVU, for giving me the opportunity to meet a lot of good and bright friends. Among all, I would like to thank Francesco Nicolo and Syvale Lee, for their continuous help and support, since the very beginning I have moved to the USA. Another special thank goes to Cesare Ciaccio, Zhengjun Wang, Peng Zheng, Tuhua Zhong, and all the other friends from the WVU International soccer team, the WVU Evansdale Roundtable, and the Keglers Wandererz's team for the recreational and fun time, and the good discussions I have had with them.

Lastly but certainly not less important, I would like to thank my parents Giovanni and Teresa Bitonti as well as my sister Giovanna who have always believed in me, and supported all my decisions. They have the capability to always cheer me up and to make each day a little better. A very special thank goes to Betsy V. Neyra, who has been the most caring, positive, supportive, patient, and the best girlfriend I could ever have. This dissertation would have never been possible without her spiritual support, and this is why I dedicate it to her. 


\section{Contents}

\begin{tabular}{ll}
\hline Acknowledgements & iv
\end{tabular}

\begin{tabular}{|l|l}
\hline List of Figures & $\mathrm{x}$
\end{tabular}

List of Tables $\quad$ xiv

\begin{tabular}{ll}
\hline List of Abbreviations & XV
\end{tabular}

\begin{tabular}{lll}
\hline Notation & $\mathrm{xx}$
\end{tabular}

\begin{tabular}{lll}
\hline & Introduction & 1
\end{tabular}

1.1 Modeling Interference in Wireless Networks . . . . . . . . . . . . . . . . . . . 2

1.1.1 Statistical Interference Models . . . . . . . . . . . . . . . . . . 5

1.1 .2 Models Describing the Effects of Interferences . . . . . . . . . . . . . 8

1.2 Techniques to Analyze the Network Performance. . . . . . . . . . . . . . . . 10

1.3 Computational Complexity in Wireless Networks . . . . . . . . . . . . . . . 12

1.4 Scope, Outline and Contributions . . . . . . . . . . . . . . . . . . 13

\begin{tabular}{|lll}
2 & Channel Outage Analysis in Wireless Networks & 16
\end{tabular}

2.1 Network Model . . . . . . . . . . . . . . . . . . . . . . . 17

2.2 Conditional Outage Probability . . . . . . . . . . . . . . . . . . . . . . . . . 18

$2.3 \quad$ Spatially Averaged Outage Probability . . . . . . . . . . . . . . . . . . 20

2.3 .1 Binomial Point Processes . . . . . . . . . . . . . . . . . . . . . . . 20

2.3 .2 Poisson Point Processes . . . . . . . . . . . . . . . . 26

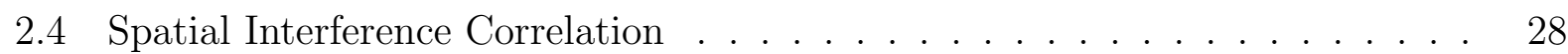

2.4 .1 Special Cases . . . . . . . . . . . . . . . . . . . . . . 29

2.5 Numerical Results . . . . . . . . . . . . . . . . . . . . . . . . . . . . . . . . . 31

2.6 Chapter Summary . . . . . . . . . . . . . . . . . . . . . . 36

\begin{tabular}{|lll}
3 & Cellular Networks: Modeling and Analysis & 38
\end{tabular}

3.1 Modeling and Analysis for Cellular Networks . . . . . . . . . . . . . . . . . . 39

3.2 Network Model . . . . . . . . . . . . . . . . . . . . . . . . . . 40

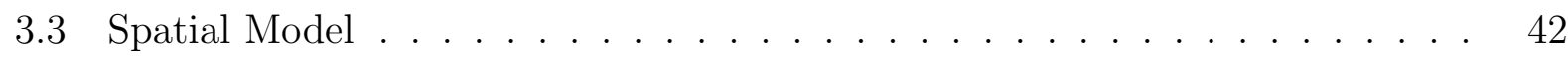

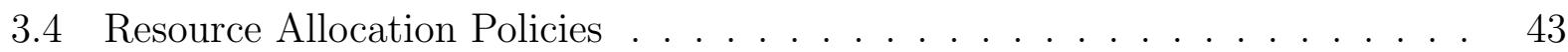

3.4 .1 Power Control . . . . . . . . . . . . . . . . . . . . . . . 43 
3.4 .2 Rate Control . . . . . . . . . . . . . . . . . . . . . 45

3.4 .3 Fixed-Rate Policies . . . . . . . . . . . . . . . . . . . . . . . 47

3.4 .4 Variable-Rate Policies . . . . . . . . . . . . . . . . . . . . . . 48

3.5 Cell Reselection . . . . . . . . . . . . . . . . . . . . . . . . . . . . . . . . . 49

3.6 Performance Analysis . . . . . . . . . . . . . . . . . . . . . . . . . . . . 49

$3.6 .1 \quad$ Outage-Constrained Fixed-Rate Policy $\ldots \ldots \ldots \ldots$. . . . . . . . 51

3.6 .2 Outage-Constrained Variable-Rate Policy . . . . . . . . . . . . . . . 51

3.6 .3 Policy Comparison $\ldots \ldots \ldots \ldots \ldots \ldots$

3.6 .4 Spreading factor $\ldots \ldots \ldots \ldots \ldots \ldots \ldots$

3.6 .5 Base-station Exclusion Zone $\ldots \ldots \ldots \ldots . \ldots \ldots$

3.6 .6 Cell Association . . . . . . . . . . . . . . . . . . . . . . . 54

3.7 Chapter Summary . . . . . . . . . . . . . . . . . . . 56

4 Diversity Combining: Outage Analysis and Applications $\quad 57$

4.1 Network Model . . . . . . . . . . . . . . . . . . . . . . . . . 58

4.2 Conditional Outage Probability $\ldots \ldots \ldots \ldots \ldots$

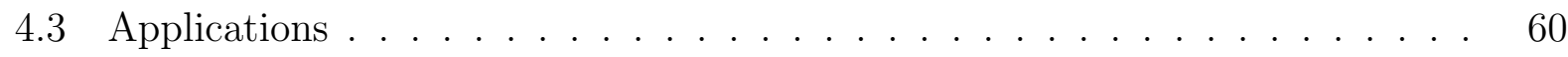

$4.3 .1 \quad$ Multi-Cell Downlink Cooperation . . . . . . . . . . . . . . 60

$4.3 .2 \quad$ MBSFN Networks. . . . . . . . . . . . . . . . . . 66

4.4 Chapter Summary $\ldots \ldots \ldots \ldots \ldots \ldots$

5 The Role of Complexity in Cellular Networks 72

5.1 Cloud-RAN: A Brief Introduction . . . . . . . . . . . . . . . . . . 73

5.2 Complexity model $\ldots \ldots \ldots \ldots \ldots \ldots \ldots \ldots$

5.2 .1 Link Adaptation $\ldots \ldots \ldots \ldots \ldots$

5.2 .2 Complexity Model $\ldots \ldots \ldots \ldots \ldots$. . . . . . . . . . 77

5.3 Performance Metrics $\ldots \ldots \ldots \ldots \ldots$. . . . . . . . . . . . . . 80

5.3 .1 Computational outage probability $\ldots \ldots \ldots \ldots \ldots$

5.3 .2 Outage complexity $\ldots \ldots \ldots \ldots \ldots \ldots$. . . . . . . . . . 80

5.3 .3 Computational gain and diversity $\ldots \ldots \ldots \ldots \ldots$

8.3 .4 Complexity-Rate Tradeoff $\ldots \ldots \ldots \ldots$. . . . . . . . . . . . . . 82

5.3 .5 Average Achievable Rate . . . . . . . . . . . . . . . . . . . . . 82

5.4 A Framework for Complexity Analysis $\ldots \ldots \ldots \ldots$

5.4 .1 Full Power Control $\ldots \ldots \ldots \ldots \ldots \ldots$

5.4 .2 Effect of Path Loss and Power Control . . . . . . . . . . . . . 85

5.4 .3 Centralized Processing . . . . . . . . . . . . . . . . . . . 86

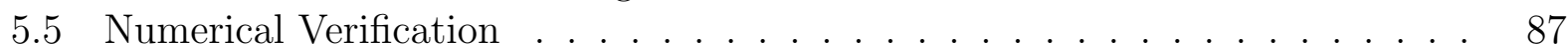

5.6 Results . . . . . . . . . . . . . . . . . . . . . . . . . . . . 90

5.6 .1 Computational Gain . . . . . . . . . . . . . . . . . . . 90

5.6 .2 Computational Diversity $\ldots \ldots \ldots \ldots \ldots$

$5.6 .3 \quad$ Complexity-Rate Tradeoff $\ldots \ldots \ldots$. . . . . . . . . . . . . . . . 92

5.7 Chapter Summary $\ldots \ldots \ldots \ldots \ldots$ 
\begin{tabular}{|lll}
\hline & Resource Management in Cloud-RANS & 95
\end{tabular}

$6.1 \quad$ Heuristic Approach to MCS Selection . . . . . . . . . . . . . . . . . 96

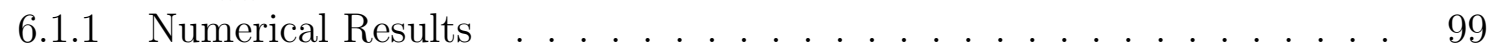

$6.2 \quad$ Optimal MCS Scheduling Policies . . . . . . . . . . . . . . . . . . . . . . 104

$6.2 .1 \quad$ Max-Rate Optimization Problem . . . . . . . . . . . . . . . 105

6.2 .2 Application to Limited Number of Rates and Multiple Users . . . . . 107

$6.2 .3 \quad$ Complexity Cut-Off $\ldots \ldots \ldots \ldots \ldots \ldots \ldots \ldots$

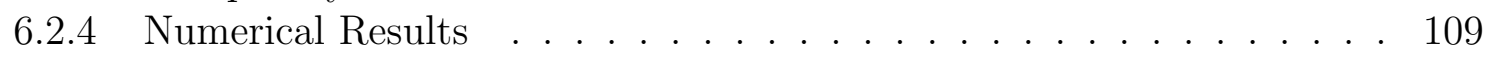

6.3 Chapter Summary . . . . . . . . . . . . . . . . . . . . . . . . . . . . 113

7 Frequency-Hopping: Optimization in Ad Hoc Networks 115

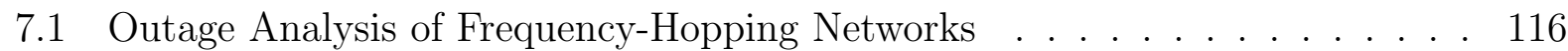

7.1 .1 Network Model . . . . . . . . . . . . . . . . . . . . . . 117

7.1 .2 Conditional Outage Probability . . . . . . . . . . . . . . . 120

$7.1 .3 \quad$ Spatially Averaged Outage Probability . . . . . . . . . . . . . 120

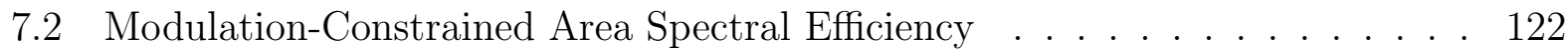

7.3 Optimization and Rate Allocation Policies _ . . . . . . . . . . . . . . . . 125

$7.3 .1 \quad$ Fixed Rate Optimization . . . . . . . . . . . . . . . . . . 126

7.3 .2 Rate Adaptation $\ldots \ldots \ldots \ldots \ldots \ldots \ldots$

7.4 Chapter Summary . . . . . . . . . . . . . . . . . . . . . . . . 134

8 Geographic Routing Protocols in Ad Hoc Networks 135

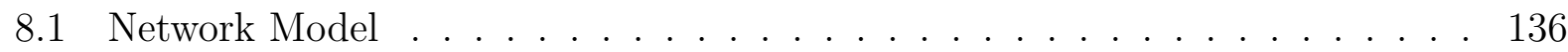

8.2 Network Simulation . . . . . . . . . . . . . . . . . . . . . . . . . . . 136

8.3 Interference Correlation $\ldots \ldots \ldots \ldots$

8.4 Routing Protocols . . . . . . . . . . . . . . . . . . . . . . . . . . . . . . 138

8.5 Implementation of Path Selection . . . . . . . . . . . . . . . . . . 140

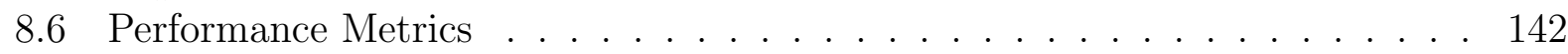

8.7 Performance Analysis . . . . . . . . . . . . . . . . . . . . . . . . . . . . 143

8.8 Chapter Summary . . . . . . . . . . . . . . . . . . . . . . . . . . . . 148

$\begin{array}{lll}9 & \text { Stochastic Modeling of Cooperative Multi-hop Wireless Networks } & 149\end{array}$

9.1 Markov Process and Multi-hop Wireless Networks . . . . . . . . . . . . 150

9.2 Application: Barrage Relay Networks _ . . . . . . . . . . . . . . . . 151

9.2 .1 Barrage Relay Network . . . . . . . . . . . . . . . . . . . . 152

9.2 .2 Controlled Barrage Regions . . . . . . . . . . . . . . . . . 153

9.2 .3 Network Model . . . . . . . . . . . . . . . . . . . . . . 155

9.2 .4 Controlled Barrage Region as a Markov Process . . . . . . . . . . 155

9.2 .5 Inter-CBR Interference $\ldots \ldots \ldots \ldots \ldots \ldots$

9.2 .6 Intra-CBR Interference . . . . . . . . . . . . . . . . . . . . 164

9.3 Chapter Summary . . . . . . . . . . . . . . . . . . . . . . . . . . . . . 169

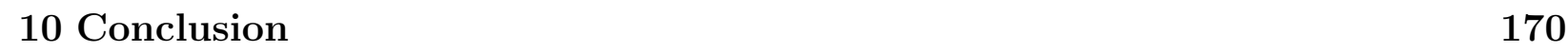

10.1 Summary and Publications . . . . . . . . . . . . . . . . . . 171

10.2 Future Work . . . . . . . . . . . . . . . . . . . . . 175 


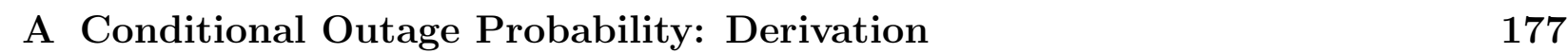

\begin{tabular}{lll}
\hline B & Asymptotic Outage Analysis & 179
\end{tabular}

2.1 Direct Approach $\ldots \ldots \ldots \ldots \ldots$. . . . . . . . . . . . . . . . . 179

2.2 Stochastic Geometry Approach . . . . . . . . . . . . . . . . . . . . 182

\begin{tabular}{ll|}
\hline C Correlation Coefficient in a BPP: Derivation & 183 \\
\hline
\end{tabular}

$3.1 \quad$ Spatially Averaged First Moment . . . . . . . . . . . . . . . . . . . . . . . . 183

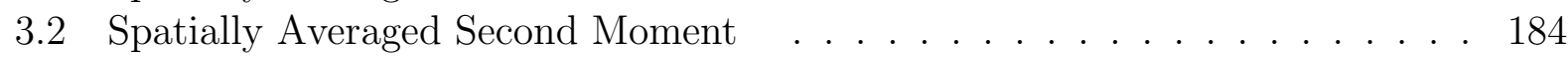

3.3 Spatially Averaged Joint First Moment . . . . . . . . . . . . . . . . . . . . 184

D Conditional Outage Probability for Diversity Combining: Derivation 186

E $\quad$ Expectation and Variance of the Decoding Complexity: Derivation 188

5.1 Expectation of the Decoding Complexity . . . . . . . . . . . . . 188

5.2 Variance of the Decoding Complexity . . . . . . . . . . . . . . . . . . . 190

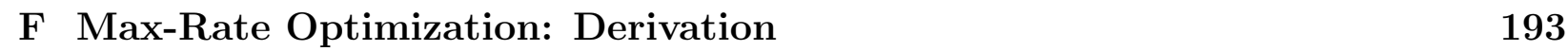

\begin{tabular}{ll}
\hline References & 195
\end{tabular}

\begin{tabular}{lr}
\hline List of Publications & 207
\end{tabular} 


\section{List of Figures}

$1.1 \quad$ Vulnerability circle capture model. . . . . . . . . . . . . . . . . . . . . 5

2.1 Typical network topology. The reference transmitter is located at the center. The two reference receivers are represented by the two red stars on the circumference of radius $r_{0} . \quad \ldots \ldots \ldots \ldots \ldots$

2.2 Conditional outage probability $\epsilon_{j}(\boldsymbol{\Omega})$ as a function of SNR. Analytical curves are solid while dots represent simulated values. Top curve: all channels Rayleigh. Middle curve: all channels $m_{i, j}=4$. Bottom curve: $m_{0, j}=4$ for the source, and $m_{i, j}=1$ for the interferers. The network topology is shown in the inset. The receiver is represented by the star at the center of the radius- 4 circle, the desired transmitter is the big red dot, while the 50 interferers are shown as blue dots. . . . . . . . . . . . . . . . . . 32

2.3 Spatially averaged outage probability for a BPP as a function of the number of interferers $M$ for two values of $p$. For each $p$, curves are shown for the case of no shadowing, and for shadowing with two values of $\sigma_{s} . \ldots 33$

$2.4 \quad$ Spatially averaged outage probability with interfering devices placed according to a PPP plotted as a function of the effective spatial density of interfering devices $\lambda p$. Curves are shown for three finite network radii $\left(r_{\text {out }}=[1,2,4]\right)$ and for the asymptotic case $\left(r_{\text {out }} \rightarrow \infty\right)$. The fading is Rayleigh, and there is no shadowing. Dots indicate the results of Monte Carlo simulation while the curves are computed from the theoretical expressions. . . . . . . . . . . . . 34

2.5 Coverage probability $\bar{\epsilon}_{j}=1-\epsilon_{j}$ as function of the network density $\lambda$. Results are shown for the case that the interferers are drawn from a BPP (solid lines) and from a PPP (dashed lines). From highest to lowest coverage probability, the curves correspond to the arbitrary shape (blue lines), the triangle (red lines), and the circle (green lines). $\ldots \ldots \ldots \ldots . \ldots . \ldots . \ldots 34$

2.6 Spatially averaged correlation coefficient as function of $\theta / \pi$. Results are shown for the case that the interferers are drawn from a BPP (solid lines) and from a PPP (dashed lines) and for three values of $\lambda p . \quad \ldots \ldots \ldots . . \ldots 35$

$3.1 \quad$ Illustration of intracell (all the UEs within the light red area) and intercell (all UEs within the orange area) interference. BSs are represented by large circles, and UEs are represented by black dots with the exception of the reference UE which is a green star. Cell boundaries are represented by thick blue lines. . . 41 
3.2 Example network topologies. BSs are represented by large circles, and cell boundaries are represented by thick lines. Left subfigure: Actual BS locations from a current cellular deployment. Right subfigure: Simulated BS locations using a BS exclusion zone $r_{\mathrm{ex}_{\mathrm{bs}}}=0.25 . \ldots \ldots \ldots$. . . . . . . . . . 43

3.3 Outage probability of eight randomly selected uplinks (dashed lines) along

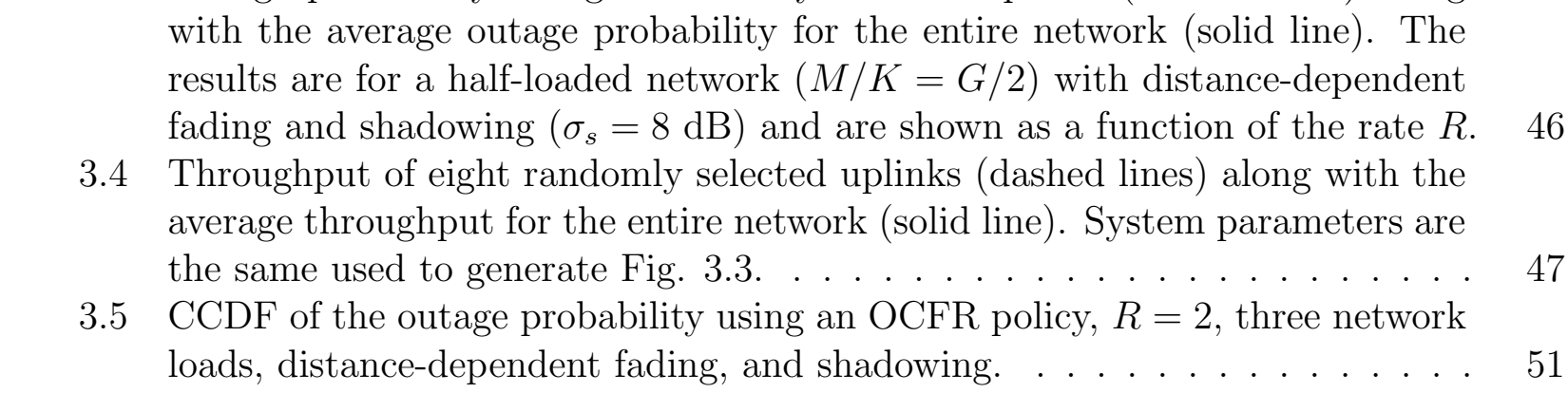

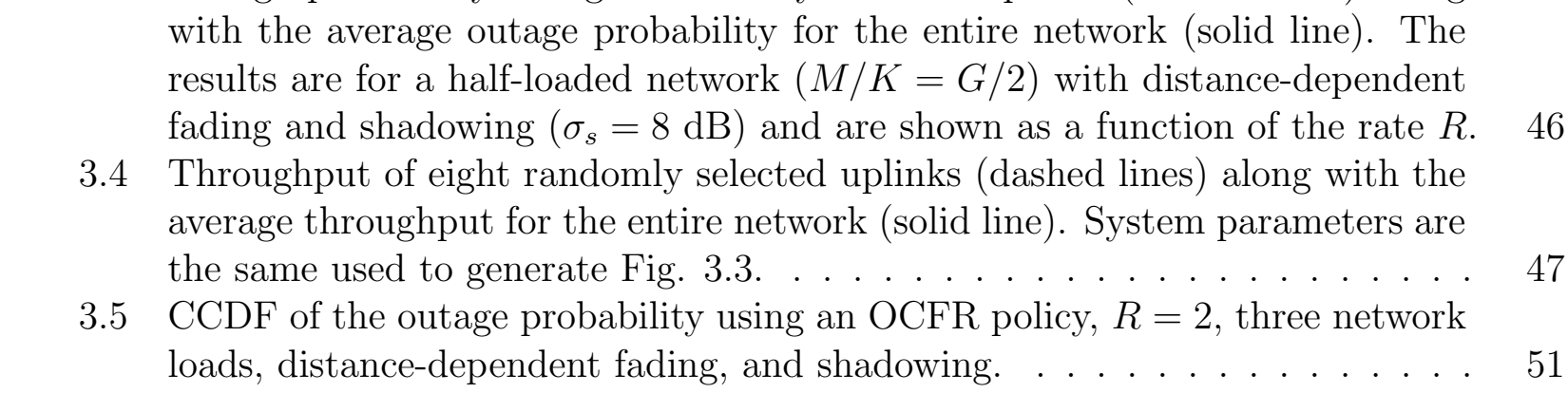

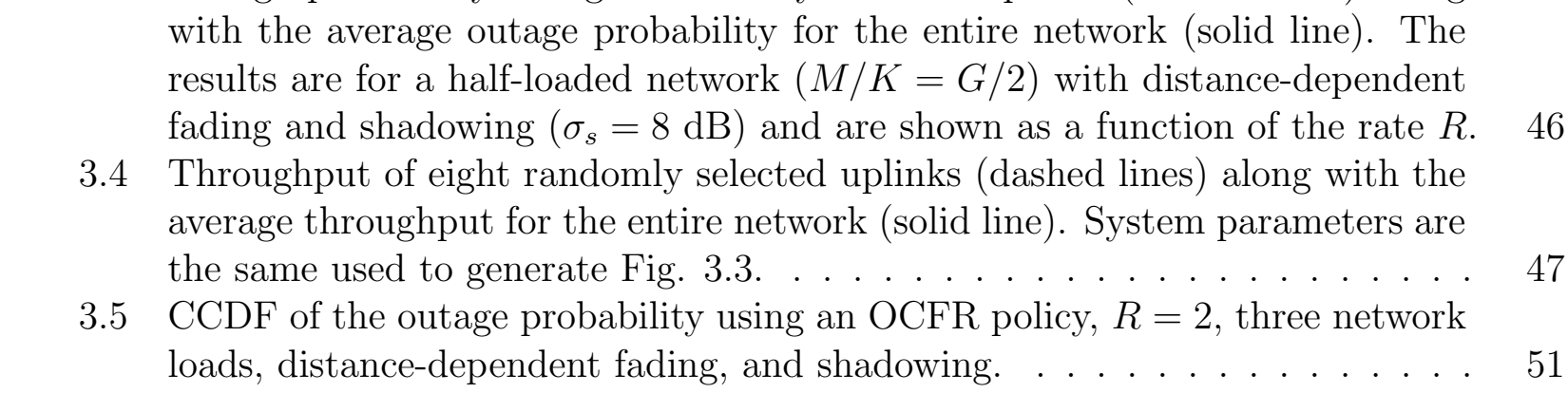

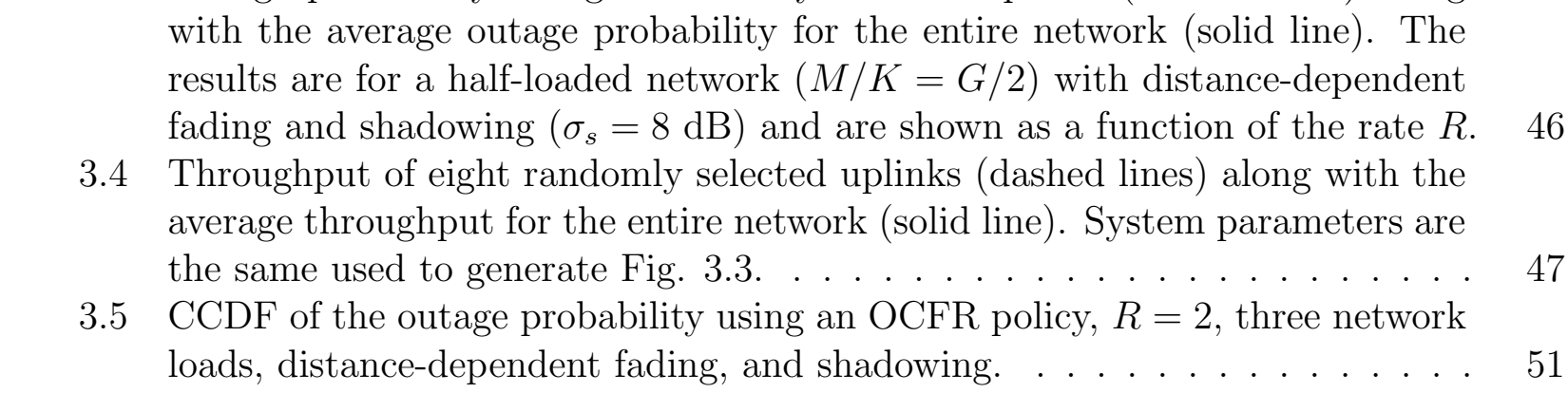

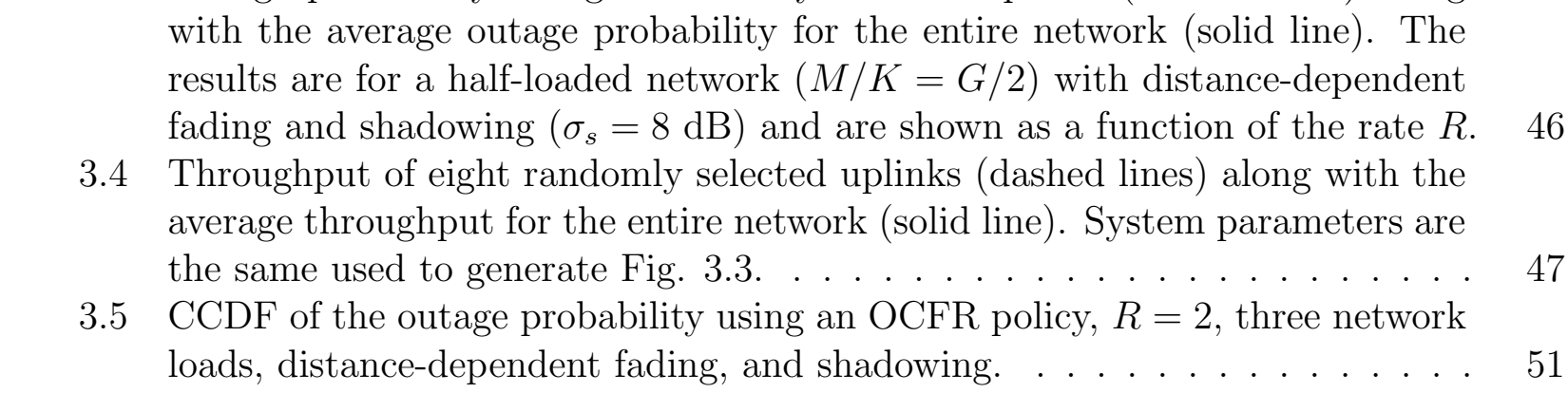

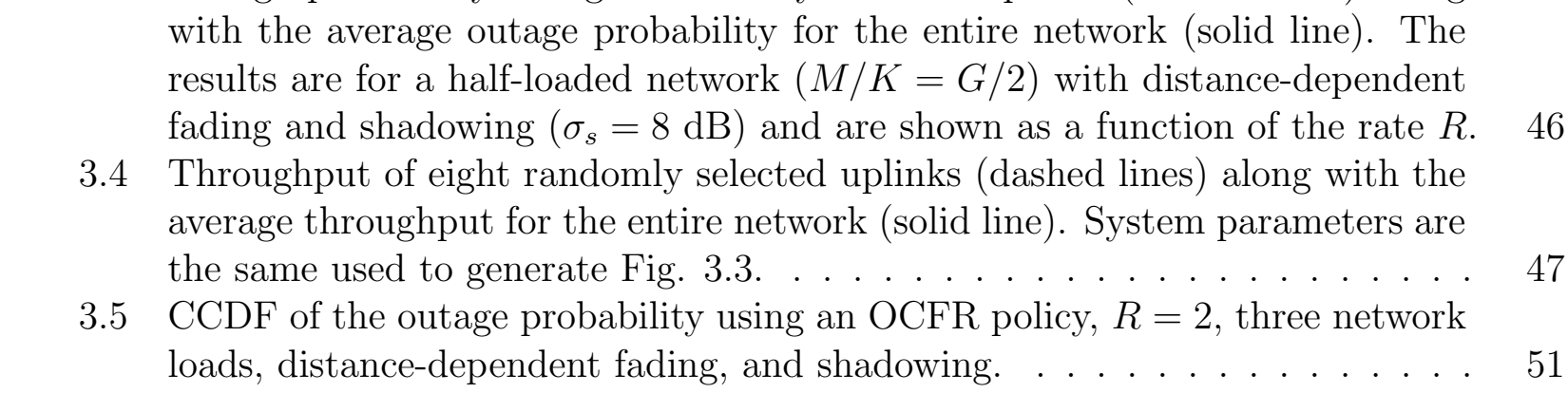

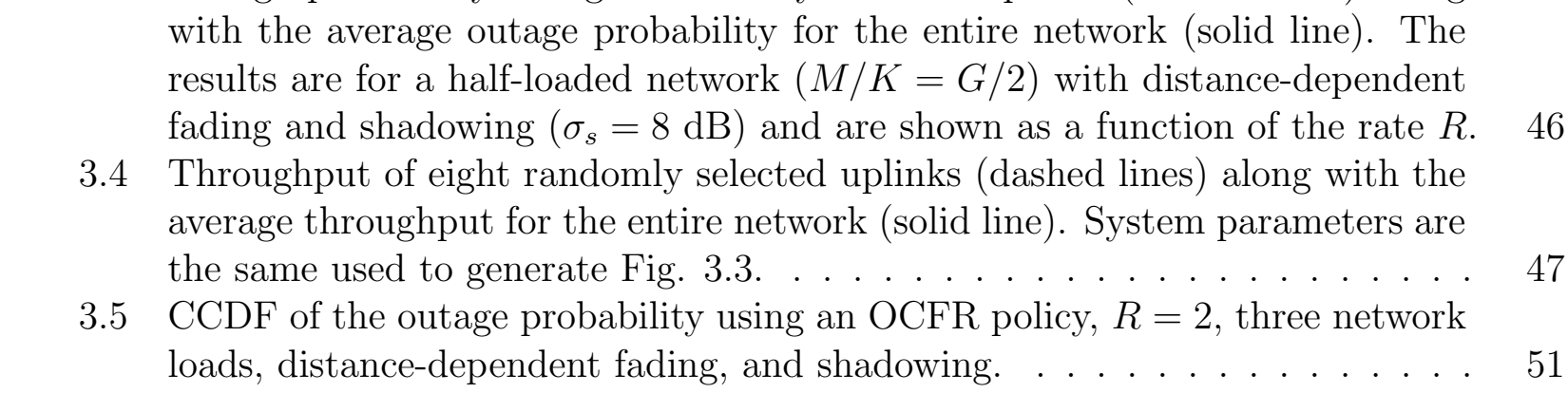

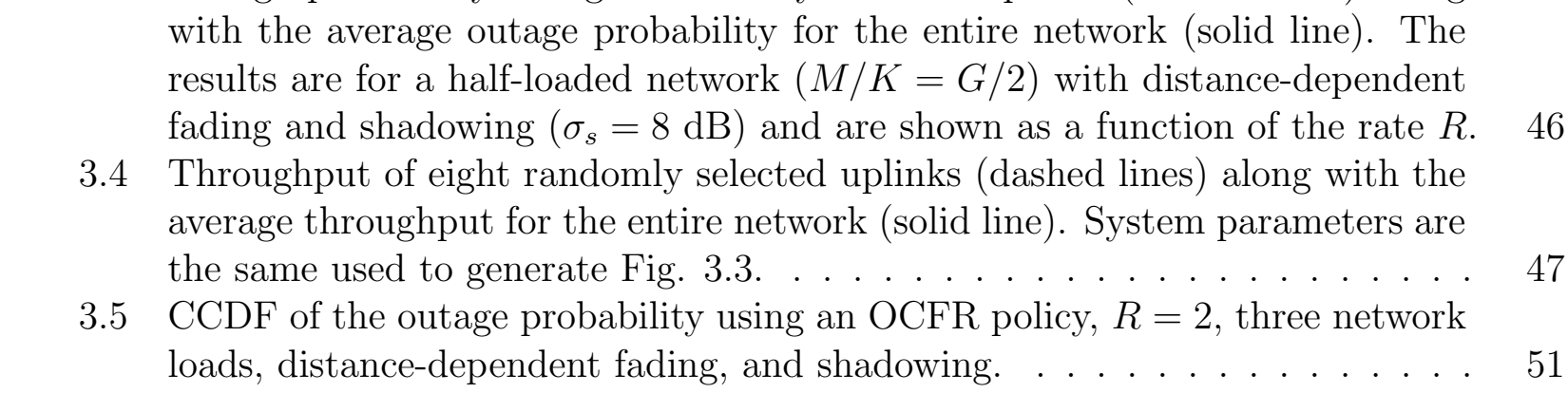

3.6 Average rate of the OCVR policy as function of the load $M / K$ for both Rayleigh and distance-dependent fading, and both shadowed $\left(\sigma_{s}=8 \mathrm{~dB}\right)$ and

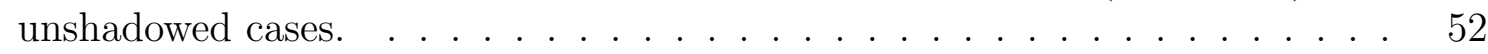

3.7 CCDF of the rate for fully-loaded system $(M / K=G)$ under the OCVR policy in Rayleigh and distance-dependent fading, and both shadowed $\left(\sigma_{s}=8 \mathrm{~dB}\right)$ and unshadowed cases. . . . . . . . . . . . . . . . . . 52

3.8 ASE for the four network policies as function of the load $M / K$ for distancedependent fading and both shadowed $\left(\sigma_{s}=8 \mathrm{~dB}\right)$ and unshadowed cases.

3.9 ASE as function of effective spreading factor $G_{e}$ for two values of system load, distance-dependent fading, and shadowing with $\sigma_{s}=8 \mathrm{~dB}$. . . . . . . . . . . 54

$3.10 \mathrm{ASE}$ as a function of the BS exclusion-zone radius $r_{\mathrm{ex}_{\mathrm{bs}}}$ for four policies and

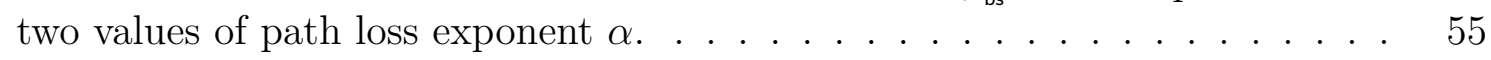

3.11 ASE as a function of the maximum reselection distance $r_{\max }$. . . . . . . 55

4.1 Illustration of the cell association: $\mathrm{UE} Y_{1}$ is only served by BS $X_{1}$, since $X_{1}$ is located at effective distance lower than $r_{\mathrm{ci}}$; UE $Y_{2}$ is instead served by a plurality of BSs within a maximum effective distance $r_{\max }$, since there is no BSs at an effective distance lower than $r_{\mathrm{ci}} . \ldots \ldots \ldots \ldots 6$. . . . . . . . 61

4.2 ASE as a function of $K / M$ with ERP and ETP policy, for both a shadowed $\left(\sigma_{s}=8 \mathrm{~dB}\right)$ and unshadowed environment. $\ldots \ldots \ldots \ldots 4$

4.3 CCDF of $R$ for ERP and ETP policy for a half loaded system $(K / M=8)$ in Shadowing $\left(\sigma_{s}=8 \mathrm{~dB}\right) . \ldots \ldots \ldots \ldots \ldots$

4.4 Close-up of an example network topology. The BSs locations are given by the large filled circles, the Voronoi tessellation shows the radio cell boundaries, and MBSFN areas are illustrated with different colors. The white areas are the portion of the network for which the outage probability is above a typical

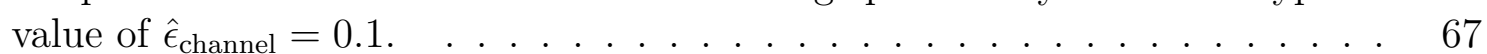

4.5 ABOT as a function of the threshold $\hat{\epsilon}_{\text {channel }}$ for both a shadowed $\left(\sigma_{s}=8 \mathrm{~dB}\right)$ and unshadowed environment with $R=0.5$. . . . . . . . . . . . . . 70

4.6 ABOT as a function of the minimum separation among BSs when $R=0.1$, $\lambda=0.1$ and $d_{\text {sfn }}=6$. 
5.1 Illustration of the Cloud-RAN architecture.

5.2 Complexity as a function of the SNR obtained both through simulations (blue) and based on (5.3) (black curve), when the maximum number of iterations used to decode a CB is limited to eight. . . . . . . . . . . . . . . . 79

5.3 Outage complexity to ensure per-cell outage constraint $\hat{\epsilon}=0.1$ as function of the number of BSs, whose signals are centrally processed. Solid lines are evaluated analytically, while dots are obtained through simulations using one million trials. The notches on the right side of each sub-figure show the behavior as $N_{\text {cloud }} \rightarrow \infty$. . . . . . . . . . . . . . . . . . . . 89

5.4 Complexity gain for $\gamma_{\mathrm{ud}}=0 \mathrm{~dB}, \alpha=2, s=0.1$. The notches on the right side

of the first sub-figure show the behavior as $N_{\text {cloud }} \rightarrow \infty$. . . . . . . . . . 91

5.5 Computational diversity as function of the target computational outage probability.

5.6 CRT as a function of $N_{\mathrm{c}}$. The notches on the right side of the figure show the behavior as $N_{\text {cloud }} \rightarrow \infty . \ldots \ldots \ldots \ldots \ldots \ldots$

6.1 Code block error rate as a function of SNR for MCS 10 and MCS 11 after 2, \begin{tabular}{|c|c|}
\hline 3 , and 8 decoder iterations. The arrow shows where the CBLER of MCS 10 \\
\hline \hline
\end{tabular} after 2 iterations is the same as that of MCS 11 after 8 iterations. . . . . . . 97

6.2 Raw throughput and computational effort as a function of the SNR for two MCS selection schemes: computationally aware selection (CAS) and max-rate selection (MRS). $\ldots \ldots \ldots \ldots \ldots$

6.3 Outage probability as a function of average SNR in the presence of Rayleigh fading, both with and without a complexity constraint. . . . . . . . . . . 100

6.4 Effective throughput as a function of average SNR in the presence of Rayleigh fading, both with and without a complexity constraint. . . . . . . . . . . . 101

6.5 Base station locations. The cloud group consists of the highlighted cells in the center of the diagram. . . . . . . . . . . . . . . . . 102

6.6 Sum throughput as function of the per-BS complexity constraint when $N_{\text {cloud }}=$ 8, with local processing (LP) and with cloud processing (CP). Two MCSselection schemes are considered: computationally aware selection (CAS) and max-rate selection (MRS). . . . . . . . . . . . . . . 103

6.7 Sum throughput with cloud processing as function of the density of UEs when $N_{\text {cloud }}=8$. Two MCS-selection schemes are considered: computationally aware selection (CAS) and max-rate selection (MRS). 
6.10 Sum-rate as a function of the user-density $\lambda$. The system is designed such that it suffers from $\epsilon_{\text {comp }}=10 \%, 0.1 \%$ computational outage in the case of $\lambda=0.5 \mathrm{UEs} / \mathrm{km}^{2}$. The curves are indicated by solid, and dash-dotted lines, respectively.

$7.1 \quad$ Illustration of the bandwidth usage in a FH spread spectrum system. . . . . 117

7.2 Power spectral density of MSK as function of the normalized frequency. Dashed lines delineate starting from the left the 95 and 99-percent power bandwidth. 119

7.3 The symmetric-information rate $C(h, \gamma)$ of noncoherent binary CPFSK with modulation indices $h=[0.2,0.4,0.6,1]$. The markers were found through Monte Carlo integration while the curves are a polynomial fit. . . . . . . . . 123

7.4 Bandwidth efficiency $B_{\text {eff }}$ as function of the modulation index $h$ for four values

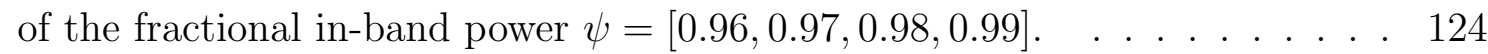

7.5 CDF of the code rate when the network is optimized. Three curves are provided: a) dense network $(M=50)$; b) moderate dense network $(M=25)$; c) sparse network $(M=5)$.

8.1 Average path reliability for both phases of each routing protocol as a function of the distance between source and destination. . . . . . . . . . . . . . . . 144

8.2 Normalized ASE of each routing protocol as a function of the distance between source and destination. . . . . . . . . . . . . . . . 145

8.3 Average conditional delay and normalized ASE of each routing protocol as a function of the maximum number of retransmissions during the message-

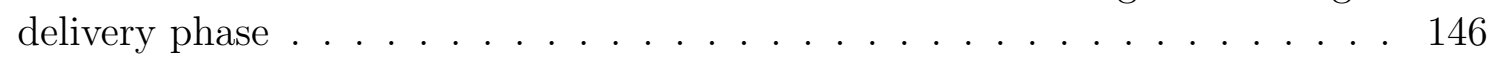

8.4 Normalized ASE of each routing protocol as a function of the effective spreading factor. . . . . . . . . . . . . . . . . . . . 147

8.5 Normalized ASE for each routing protocol as a function of the contention density with the relay density as a parameter. . . . . . . . . . . . . . 148

9.1 Example of Barrage relay networs (BRNs), composed of multiple controlled barrage regions (CBRs). Each CBR is composed a four-node network. . . . . 153

9.2 Markov chain for a CBR composed of four nodes $(N=2)$ and for the duration of three time slots. Transient states are in white, while the CBR success absorbing state is in green and the CBR outage absorbing state is in red. Each of the two absorbing states is the union of several CBR states. . . . . . 156

9.3 CBR outage probability as a function of the SNR when CCI is neglected. The solid lines are obtained analytically while the markers are obtained from Monte Carlo simulation. The network topology is a four node line network shown in the inset, and $\alpha=3.5$.

\begin{tabular}{|c|c|}
\hline & The solid lines are obtained analytically while the markers are obtained from \\
\hline & "Monte Carlo simulation. The network topology is a four node line network \\
\hline & shown in the inset, and $\alpha=3.5 . \ldots \ldots \ldots \ldots \ldots$ \\
\hline 9.4 & CBR outage probability for the $k^{t h}$ CBR as function of the number of itera- \\
\hline & tions used, when only the inter-CBR interference is considered. Set of curves \\
\hline & at the top: $S N R=0 \mathrm{~dB}$. Set of curves at the bottom: $S N R=10 \mathrm{~dB}$. Each set \\
\hline & of curves is obtained using three values of $\alpha . \ldots \ldots \ldots$ \\
\hline 9.5 & CBR outage probability for the $k^{t h} \mathrm{CBR}$ as function of the number of radio \\
\hline & frames considered, when only the intra-CBR interference is taken into accoun \\
\hline
\end{tabular}




\section{List of Tables}

1.1 Common spatial point processes. . . . . . . . . . . . . . . . 6

7.1 Optimal parameter values when ACI due to spectral splatter is ignored, and

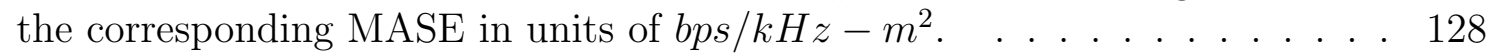

7.2 Optimal parameter values when ACI due to spectral splatter is considered,

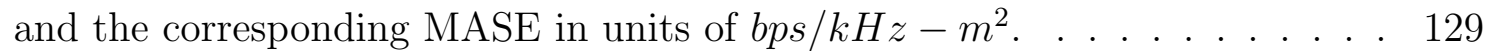

7.3 Optimal parameter values when ACI due to spectral splatter is considered, and the corresponding MASE in units of $b p s / k H z-m^{2}$. . . . . . . . . . 133

$9.1 \quad$ Optimization results when inter-CBR interference is considered. . . . . . . . 163

$9.2 \quad$ Optimization results when intra-CBR interference is considered.. . . . . . . 168 


\section{List of Abbreviations}

ABOT area below an outage threshold

ACI adjacent-channel interference

AODV ad hoc on-demand distance-vector

ARQ automatic repeat request

ASE area spectral efficiency

AWGN additive white Gaussian noise

BBU baseband unit

BPP binomial point process

BRN Barrage relay network

BS base station

CAS computationally aware selection

CB code block

CBLER code block error rate

CBR controlled barrage region

CCDF complementary cumulative distribution function

CCI co-channel interference

CDF cumulative distribution function 
CDMA code division multiple access

CF characteristic function

CoMP coordinated multi-point

CP centralized processing

CPP cluster process process

CPFSK continuous-phase frequency-shift keying

C-RAN cloud/centralized radio access network

CRC cyclic redundancy check

CRT complexity-rate tradeoff

CSI channel state information

CSMA carrier sense multiple access

CSMA/CA carrier sense multiple access with collision avoidance

CTS clear-to-send

DDO denial due to overload

DS direct-sequence

DS-CDMA direct-sequence code division multiplex-access

DU data unit

ERP equal received power

ETP equal transmit power

FDMA frequency division multiple access

FEC forward error correction

FFT fast Fourier transform 
FH frequency-hopping

FHMA frequency-hopping multiple access

FLOPS floating-point operations per second

FT Fourier transform

GF greedy forwarding

HARQ hybrid automatic repeat request

HCPP hard core point process

ISI inter-symbol interference

IT information technology

LDPC low-density parity-check

LOS line-of-sight

LP local processing

LT Laplace transform

LTE Long Term Evolution

MAC medium access control

MANET mobile ad hoc network

MASE modulation-constrained area spectral efficiency

MBMS multimedia broadcast multicast service

MBSFN multicast-broadcast single-frequency network

MCC multi-cell cooperation

MCE multi-cell/multicast coordination entity

MCS modulation and coding scheme 
MIMO multiple-input and multiple-output

MLSE maximal-likelihood sequence estimation

MP maximum progress

MRC maximal-ratio combining

MRS max-rate selection

MSK minimum-shift keying

MTFR maximal-throughput fixed rate

MTVR maximal-throughput variable-rate

OCFR outage-constrained fixed rate

OCVR outage-constrained variable-rate

OFDMA orthogonal frequency-division multiple access

PCP Poisson cluster process

PDF probability density function

PDP power delay profile

PGFL probability generating functional

PPP Poisson point process

PMF probability mass function

RAN radio access network

RB resource block

RDO reselection due to overload

$\mathbf{R E}$ resource element

$\mathbf{R R H}$ remote radio head 
RTS request-to-send

SCC scheduling with complexity cut-off

SC-FDMA single-carrier frequency division multiple access

SFN single-frequency network

SINR signal-to-interference-and-noise ratio

SNR signal-to-noise ratio

SWF scheduling with water-filling

TB transport block

TBLER transport block error rate

TC transmission capacity

TDMA time division multiple access

UCP uniform cluster process

UE user equipment

VM virtual machine

VTE vehicular test environment 


\section{Notation}

The following notation and symbols are used throughout this dissertation.

$\|\cdot\| \quad:$ Euclidian norm

$|\cdot|$ : Cardinality of a set

$|\mathcal{A}| \quad:$ Network area

$\alpha \quad$ : Path loss exponent

$B \quad$ : Spectral band in $\mathrm{Hz}$

$\beta \quad$ : Signal-to-interference and noise ratio (SINR) threshold

c : Speed of light

C : Channel capacity

$\mathcal{C} \quad$ : Decoding complexity

$c_{f} \quad:$ Chip factor

$\mathcal{C}_{\max }:$ Maximum computational resources allocated to a base station

$C_{k} \quad:$ Number of code blocks for the $k^{\text {th }}$ MCS

$\overline{\mathcal{D}} \quad$ : Averaged conditional delay

D : Duty factor

$\operatorname{diag}(\cdot) \quad$ : Diagonal matrix

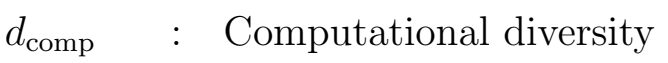

$D_{k} \quad$ : Number of information bits the $k^{\text {th }}$ MCS

$d_{\text {net }} \quad$ : Length of the side of a square area

$d_{0} \quad:$ Reference distance

$\delta(x) \quad$ : Dirac delta function

$\Delta \gamma \quad$ : Signal-to-noise ratio margin

$\epsilon_{\text {all }} \quad:$ Overall outage, which includes both channel and computational outage 
$\epsilon_{\text {comp }} \quad:$ Computational outage

$\epsilon_{j} \quad:$ Channel outage probability at the $j^{\text {th }}$ receiver

$\hat{\epsilon}_{\text {channel }}:$ Channel outage constraint

$\mathbb{E}_{X}[\cdot] \quad$ : Expectation operator respect to the random variable $x$

$\eta \quad$ : Spectral efficiency

$f_{\mathrm{fpc}} \quad:$ Fractional power-control factor

$f_{p} \quad:$ Fraction of the power dedicated to synchronization and channel estimation

$f_{x}(x) \quad$ : Probability density function $(\mathrm{PDF})$ of a random variable $x$

$F_{x}(x)$ : Cumulative Distribution Function (CDF) of a random variable $x$

G : Processing gain

$\gamma \quad:$ Instantaneous signal-to-interference and noise ratio (SINR)

$g_{\text {comp }} \quad$ : Computational gain

$g_{i, j} \quad: \quad$ Power gain between for the link between the $i^{\text {th }}$ transmitter and the $j^{\text {th }}$ receiver

$h \quad$ : Modulation index for a CPFSK modulation

$\overline{\mathcal{H}} \quad$ : Averaged number of hops

$K \quad$ : Number of UE within the network

$L \quad$ : Number of sides in a regular polygon

$L_{\max } \quad$ : Maximum number of turbo iterations

$\mathcal{L} \quad:$ Number of contiguous frequency channels

$\lambda \quad$ : Density of interferers

$M \quad$ : Number of interferers

$m_{i, j} \quad:$ Nakagami-m factor for the link between the $i^{\text {th }}$ transmitter and the $j^{\text {th }}$ receiver

$\mathcal{N} \quad:$ Noise power

$\mu \quad$ : Service probability

$N_{\text {cloud }} \quad$ : Number of jointly processed base stations

$N_{\mathrm{tr}} \quad$ : Maximum number of retransmissions allowed

$\Omega_{i, j} \quad:$ Normalized received power between the $i^{\text {th }}$ transmitter and the $j^{\text {th }}$ receiver

$p \quad:$ Probability of occurrence of a given event

$P_{i} \quad:$ Transmit power from the $i^{\text {th }}$ transmitter

$p_{x}[x] \quad$ : Probability mass function (PMF) of a random variable $x$

$\psi \quad$ : Fractional in-band power

$R \quad$ : Code rate 
$\overline{\mathcal{R}} \quad$ : Averaged path reliability or probability of end-to-end success

$r_{i, j} \quad:$ Distance between the $i^{\text {th }}$ transmitter and the $j^{\text {th }}$ receiver

$\rho[x, y] \quad$ : Correlation coefficient between random variable $x$ and $y$

$r_{\mathrm{ex}} \quad:$ Exclusion radius

$r_{\text {in }} \quad:$ Radius of a circular exclusion zone

$r_{\max }:$ Maximum connection distance

$r_{\text {out }}:$ Radius of a circular network

$r_{\mathrm{t}} \quad:$ Transmission range

$s \quad:$ Compensation factor for fractional power control

$S_{\text {mbsfn }}:$ Number of MBSFN areas

$S_{\text {sector }}:$ Number of sectors for a BS

$\overline{\mathcal{S}}_{\text {ase }} \quad:$ Averaged area spectral efficiency

$\sigma_{s} \quad$ : Standard deviation for log-normal shadowing

T : Throughput

$T_{c} \quad$ : Chip period

$T_{\mathrm{ECP}} \quad$ : Extended cyclic prefix

$t_{\text {comp }} \quad:$ Complexity-rate tradeoff

$\operatorname{tr}(\cdot) \quad$ : Trace operator

$u(x) \quad$ : Step function

$\xi_{i, j} \quad: \quad$ Log-normal shadowing coefficient between the $i^{\text {th }}$ transmitter and the $j^{\text {th }}$ receiver

Bold case letters denote vectors. 


\section{Chapter 1}

\section{Introduction}

As mobile data demand continues to increase exponentially, wireless networks need to prepare for $1000 X$ traffic growth over the next decade [1]. In order to meet the needs of future systems, novel technologies have been proposed including very densely deployed smallcell networks, Cloud-radio access networks (RANs), and cooperative communication protocols and strategies. Future networks [2] are expected to be densely deployed, with a high degree of cooperation among nodes, and empowered by the use of virtualization and cloud computing, through the use of centralized cloud platforms, which will be used to coordinate cooperative transmissions and be able to jointly process signals from multiple base stations $(\overline{\mathrm{BS}} \mathrm{s})$. In these novel networks, since both radio spectrum and computational resources allocated are finite, both the effect of the interference and the computational resources available will play a very important role. Therefore, analysis, modeling and estimation of them is very important for a better understanding of these networks and for their better and more efficient design.

This dissertation focuses primarily on four aspects: 1) It models and analyzes the effect of the interference, and introduces a novel analytical framework to compute the outage probability for wireless networks with interferers located according with a point process when the signal is subject to fading. This framework is applied to a variety of cases of study in order to prove its wide applicability and its benefits in modeling both conventional and cooperative networks; 2) It provides a better understanding of the interplay between available computing resources and the required communications performance by building a framework that estimates the required data processing resources under a given performance 
constraint for a Cloud-RAN. It also focuses on the resource management through the design of computationally aware schedulers, which improve the overall performance by accounting for the penalty in the quality of communications when the computational resources available for processing the communication signals is limited; 3) It describes a novel methodology to optimize multiple-access networks, including frequency-hopping (FH) systems, which will be more widely employed in future networks with the aim to further mitigate the effect of frequency-selective fading and prevent the near-far problem. The optimization procedure accounts for the presence of fading and interference effects, including co-channel interference (CI $)$ and adjacent-channel interference (ACI); 4) It provides a more efficient method to stochastically model and study the performance of cooperative multi-hop wireless networks, in presence of interference.

In order to provide to the reader some basic background and fundamentals, the remainder of this chapter gives a brief overview on modeling interference in wireless networks, a summary of the existing methodologies used to analyze the effect of interference, and a brief discussion on computational complexity in wireless networks. The chapter concludes by providing an outline of this dissertation, together with a brief summary of the main contributions.

\subsection{Modeling Interference in Wireless Networks}

A wireless communication network can be seen as a group of nodes, which can act as transmitters or receivers, and they are located in a given arena. At a given time, some of the nodes transmit simultaneously, in order to communicate with one or multiple receivers (in cooperative systems each receiver can be served by a plurality of transmitters). The signal propagates from each transmitter to all receivers through a given wireless link, which may be jammed by the signals from the other transmitters.

The first step to model a wireless network is to create an interference model [3]. An interference model can be viewed as the combination of the following components [4]:

1. Propagation model: This describes the effects of radio propagation on the signal as it travels through the transmission media (e.g., over the air). The three main propagation 
effects considered when modeling wireless channels are [5]:

(a) Path loss: This models the attenuation suffered by signal while traveling from the transmit antenna to the receiver antenna. A common model is $P_{r} / P_{t} \propto r^{-\alpha}$, where $P_{r}$ and $P_{t}$ are the average transmit and receive power levels, respectively, $r$ is the transmitter-receiver separation distance, and $\alpha$ is the path loss exponent.

(b) Large-scale fading: This characterizes the obstructions from large objects which cause the average received power level to vary, for the same transmitter-receiver separation distance. The large-scale fading is often called shadowing.

(c) Small-scale fading: In a typical wireless communication environment, propagation occurs through multiple paths between transmitter and receiver, and several replicas of the transmitted signal reach the receive antenna. These replicas combine with each other in a constructive or destructive way, resulting in a received signal with rapid envelope fluctuation. The most common statistical distributions used to model this phenomena include the Rayleigh, Nakagami-m and Ricean distributions.

In a typical wireless environment, all three propagation effects described above are present, such that the average power of the received signal decreases as the transmitterreceiver separation distance increases, combined with fluctuations due to small scale and large scale fading. However, depending on the propagation environment or the scenario of application, some of these effects can be neglected.

2. Spatial model: This specifies how terminals, including the desired transmitters, reference receivers, and interfering transmitters, are distributed over the network area. The model varies from a purely random distribution to an arbitrary terminal placement.

3. Traffic model: This describes the transmitter activity (when and which terminals access the radio channel), and it is mainly dictated by the medium access control (MAC) technique adopted in the network. The MAC techniques can be classified as random access (e.g., CSMA and Aloha) or scheduled access (e.g., TDMA, CDMA and FDMA). 
In an interference model the components listed above can be either a deterministic or a random process based on the scenario considered.

One of the key aspects that must be considered when modeling interference in wireless communication is how interference effects the reception of a given desired signal. In this case two models can be adopted:

1. Collision channel model [6]: This model assumes that if two or more messages are transmitted at the same time, all messages are lost due to collision, regardless of their transmit power levels.

2. Capture channel model [7]: This model, which is more realistic, assumes that successful reception may occur if the received signal is sufficiently stronger than the interfering signals. Two different versions of the capture channel model can be found in the literature, which are:

(a) Vulnerability circle capture model [8]: According to this model, a message is correctly received if its received power is larger than the individual received power of any other message by a factor $\beta_{v}$. Under the assumption that the path loss channel model is deterministic and all nodes transmit with the same power level, the above condition is equivalent to state that only transmitters within a vulnerability circle of radius $r_{v}=\beta_{v}^{\frac{1}{n}} r$ and centered at the receiver cause interference to the signal from a link transmitter located at distance $r$ from the receiver ${ }^{1}$, as illustrated in Fig. 1.1.

(b) Power capture model: This model states more realistically that a message is successfully decoded if the received power from the $i^{\text {th }}$ transmitter $P_{i}$ is at least $\beta$ times higher than the aggregated received power from all other transmitters summed with the noise power $\mathcal{N}$. In other words, the signal-to-interference-andnoise ratio (SINR) of the desired message needs to satisfy a threshold $\beta$ :

$$
\gamma=\frac{P_{i}}{\mathcal{N}+\sum_{j \neq i} P_{j}} \geq \beta
$$

\footnotetext{
${ }^{1}$ Note that the vulnerability circle capture model reduces to the collision channel model if $\beta_{v} \rightarrow \infty$.
} 


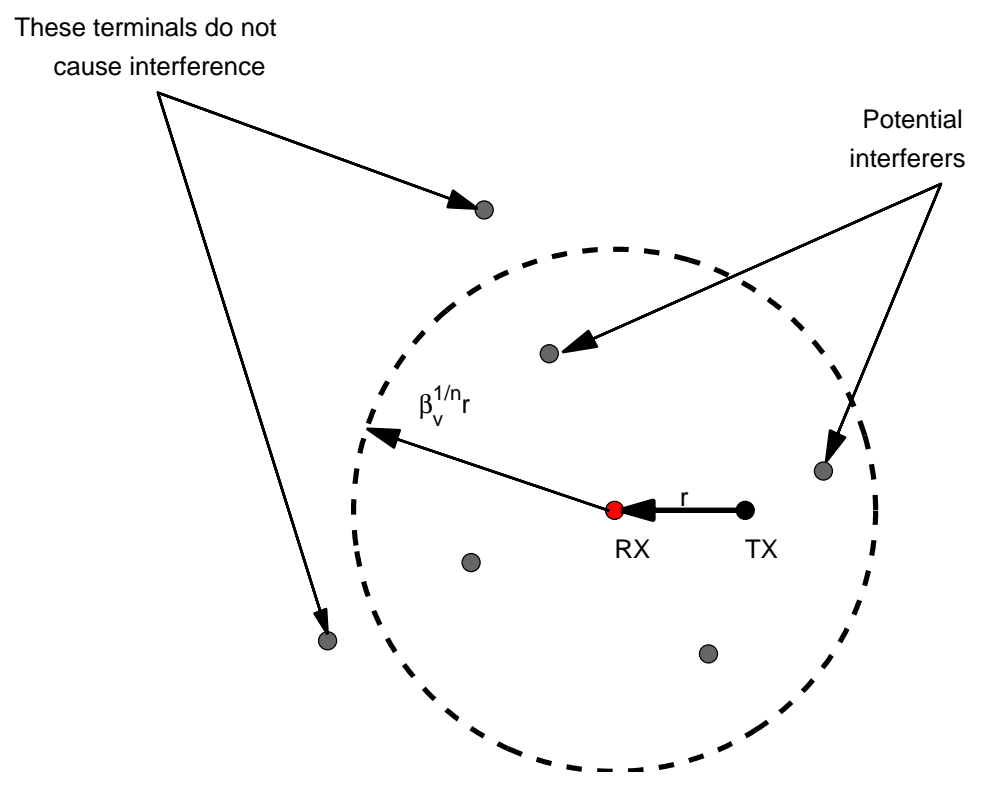

Figure 1.1: Vulnerability circle capture model.

According to how the interference is described and used, the interference models used in the literature for a wireless network can be classified into three large groups:

- statistical interference models;

- models that describe the effects of interference;

- graph-based interference models.

\subsubsection{Statistical Interference Models}

Statistical interference models are preferred when the randomness of the propagation channel gains and the randomness of user activity are considered. These models assume that the statistical characteristics of the interference depend on the statistics of the individual interfering signals, such as the propagation effects, the interferer locations and the user activity. In these models, the location of interferers relative to the receiver (or receivers) plays an important role, since these distances determine the average power levels of the interference signals. Two major classes of statistical interference models can be identified, according to the nature (stochastic or arbitrary) of interferer location: 


\begin{tabular}{|l|l|l|}
\hline Point Process & Key Properties & Practical Example \\
\hline Poisson (PPP) & $\begin{array}{l}\text { Independency between node } \\
\text { locations }\end{array}$ & $\begin{array}{l}\text { Ad hoc networks with random } \\
\text { channel access }\end{array}$ \\
\hline Hard core (HCPP) & $\begin{array}{l}\text { Minimum distance between } \\
\text { nodes }\end{array}$ & $\begin{array}{l}\text { Carrier sensing wireless net- } \\
\text { works with collision avoidance }\end{array}$ \\
\hline Cluster (CPP) & $\begin{array}{l}\text { Clusters of nodes, with inde- } \\
\text { pendence between cluster lo- } \\
\text { cations }\end{array}$ & $\begin{array}{l}\text { Sensor networks, and urban } \\
\text { networks with dense hotspots }\end{array}$ \\
\hline Binomial (BPP) & $\begin{array}{l}\text { Independency between node } \\
\text { locations, which are fixed } \\
\text { number in a given area }\end{array}$ & $\begin{array}{l}\text { A known number of relays or } \\
\text { mobile users deployed at ran- } \\
\text { dom in a cell of known size }\end{array}$ \\
\hline
\end{tabular}

Table 1.1: Common spatial point processes.

1. For the first kind of statistical interference model, the key source of randomness in the interference is the random nature of interferer locations. For this type of statistical interference model, the locations of the interferers are modeled according to a random point process. In the following, the most common point processes are described below, and their main characteristics are summarized in Table 1.1:

(a) The Poisson point process $(\overline{P P P})$ has been by far the most popular spatial model due to its analytical tractability. It can be used to model a practical situation where transmitters and/or receivers are located or moved around randomly over a large area. Indicate with $\lambda$ the intensity of the process, which is the average number of points per unit area. In a $\mathrm{PPP}$, the number of points within a given area $\mathcal{A}$ is Poisson distributed with density $\lambda$ and the average number of points is $\lambda|\mathcal{A}|$. The probability that there are $n$ points in $\mathcal{A}$ is equal to $(\lambda|\mathcal{A}|)^{n} \frac{e^{-\lambda|\mathcal{A}|}}{n !}$. Each point is placed independently and uniformly in the area $\mathcal{A}$.

(b) Even if a $\mathrm{PPP}$ can be used to model a wide range of scenarios, there are some real-world situations that requires a different model. When the channel access is not purely random (e.g., carrier sense multiple access with collision avoidance (CSMA/CA ), a more regular structure for the interference locations is required and in this case a Matérn hard core point process ( 
9 11. A HCPP is a repulsive point process, where all its points are placed such a way they are at least at distance $r_{e x}$ from each other [12]. A Matérn type of HCPP, which is obtained transforming a PPP into a HCPP by applying a thinning operation. Among the different types of Matérn $\mathrm{HCPP}_{3}$, the most used are:

- Matérn HCPP type I: Starting from a $\mathrm{PPP}$ type I is constructed by deleting all the points from the $\mathrm{PPP}$ that co-exist within a distance less than the hard core parameter $r_{e x}$. For this point process its spatial intensity $\lambda_{r_{e x}}$ is a function of the spatial intensity $\lambda$ of the initial $\mathrm{PPP}[13$ :

$$
\lambda_{r_{e x}}=\lambda e^{-\pi \lambda r_{e x}^{2}}
$$

- Matérn HCPP type II: In the type II Matérn HCPP each point of the PPP has a random mark, which is an independent uniform random variable. A point is deleted if only if there is another point of the PPP that lies within distance $r_{e x}$ with a smaller mark. The spatial intensity $\lambda_{r_{e x}}$ of the Matérn HCPP can be obtained by the Slivnyak-Mecke's theorem [14] as function of the spatial intensity $\lambda$ of the initial $\mathrm{PPP}$.

$$
\lambda_{r_{e x}}=\frac{1-e^{-\pi \lambda r_{e x}^{2}}}{\pi r_{e x}^{2}} .
$$

(c) In a binomial point process (BPP), similar to a $\mathrm{PPP}$, the distribution of the given number of points is independently and identically distributed, but unlike a PPP the number of points is fixed. In the case the size of the network is fixed and the number of relays or mobile users is known, a $\mathrm{BPP}$ is more realistic [15.

(d) Another common branch of point processes is the cluster process process (CPP), which has been frequently used in the field of communication to model urban networks with dense hotspots or in sensors networks [16].

One of the most used $\mathrm{CPP}$ is a Poisson cluster process ( $(\overline{P C P})$, which is obtained by placing points using a $\overline{\mathrm{PPP}}$ with intensity $\lambda$. Each of these points, called an immigrant [17], generates a cluster, which is itself a finite point process. Given the immigrants, the clusters are independent and identically distributed. 
Another commonly used $\mathrm{CPP}$ is the uniform cluster process (UCP), where the number of relays or mobile users placed inside the network are fixed. In a $\overline{\mathrm{UCP}}$, as $\mathrm{a} \mathrm{HCPP}$, the points are located in such a way that they are $r_{e x}$ a part each other, and for each point this region, where no other points can lie, is called exclusion region. In particular a $\overline{\mathrm{UCP}}$ can be obtained placing the points in the network arena in sequence and the location of each new point is re-assigned as many times as necessary until it does not fall within any exclusion regions of the existing points.

When a statistical interference model is adopted in random networks, stochastic geometry [18 21], which is a rich branch of applied probability, is a valid and recently widely used tool. Stochastic geometry was initially adopted for applications in biology, astronomy and material science, but nowadays it is extensively used in the context of communication networks. Stochastic geometry is particularly efficient and relevant to large-scale networks, when the statistical interference model is ergodic. In this case, stochastic geometry allows (by averaging over all possible topologies) to capture the key dependencies of the network performance characteristics (connectivity, stability, capacity, etc.) as functions of a relatively small number of parameters: typically they are the densities of the point processes and the parameters of the protocols involved.

2. Another branch of statistical interference models is composed of the interference models in arbitrary networks. In these models, the locations of the interferers is not random, but rather are fixed, and typically the propagation effects are the key source of randomness of interference. The problem of statistically characterizing interference for these models is based on deriving the statistics of the aggregate interference. In addition to the assumption that interfering signals sum incoherently, often it is also assumed that all interfering signals suffer from the same type of fading.

\subsubsection{Models Describing the Effects of Interferences}

An alternative approach in modeling wireless networks is to adopt models that describe the effects of interference, rather than modeling interference itself. Basically, these models 
focus on a specific aspect of the behavior of the network that is affected by interference. The two most popular interference models of this class are:

1. Protocol interference model [22]: This model is based on the vulnerability circle capture model and it defines a condition for successful communication over a given link. In particular, it is assumed a simple deterministic path loss channel model, and that all terminals transmit with the same power level $P_{0}$. Under these assumptions, it is possible to successfully receive a message from the $i^{\text {th }}$ transmitter if:

$$
\frac{P_{i}}{P_{j}} \geq \beta_{v} \Rightarrow \frac{P_{0}\left\|X_{i}-X_{R(i)}\right\|^{-\alpha}}{P_{0}\left\|X_{j}-X_{R(i)}\right\|^{-\alpha}} \geq \beta_{v} \Rightarrow\left\|X_{i}-X_{R(i)}\right\| \geq \beta_{v}^{-1 / \alpha}\left\|X_{j}-X_{R(i)}\right\|
$$

where $X_{i}$ denotes the $i^{\text {th }}$ terminal and its position, $X_{R(i)}$ is the intended receiver of transmissions from the $i^{\text {th }}$ terminal $X_{i}$, and $\left\|X_{i}-X_{R(i)}\right\|$ is the Euclidian distance between terminals $X_{i}$ and $X_{R(i)}$. Equation (1.4) indicates that successful transmissions require disjointed circular exclusion regions around receivers $X_{R(i)}$.

2. Physical interference model [22]: This model is based on the power capture model and, as with the protocol interference model, a simple deterministic path loss channel model is assumed. Furthermore it is assumed that all terminals transmit with the same power level $P_{0}$. According to the physical interference model a transmission from terminal $X_{i}$ is successfully received by terminal $X_{R(i)}$ if the SINR at $X_{R(i)}$ is equal to or larger than a given threshold $\beta$ :

$$
\gamma=\frac{P_{0}\left\|X_{i}-X_{R(i)}\right\|^{-\alpha}}{\mathcal{N}+\sum_{j \neq i} P_{0}\left\|X_{j}-X_{R(i)}\right\|^{-\alpha}} \geq \beta
$$

This interference model can then be generalized assuming that the aggregate interference follows a Gaussian distribution. In this way the highest data rate $R_{i}$ over the link $\left(X_{i}, X_{R(i)}\right)$ is function of the $\gamma$ according to the Shannon's capacity formula for additive white Gaussian noise (AWGN) channel. In this case the generalized physical interference model is written as 23]:

$$
R_{i}=B \log _{2}\left(1+\frac{P_{i}\left\|X_{i}-X_{R(i)}\right\|^{-\alpha}}{N_{0} B+\sum_{j \neq i} P_{j}\left\|X_{j}-X_{R(i)}\right\|^{-\alpha}}\right)
$$

where $N_{0} / 2$ is the noise spectral density, $B$ is the channel bandwidth. 
3. Graph-based Interference Models [24]: Wireless multi-hop networks are often modeled by employing graph theory, which allows to provide a representation of the network and the interconnections among nodes. A graph is a representation of a set of objects, where some pairs of objects can be connected each other by links. The interconnected objects are represented by mathematical abstractions called vertices, and the links that connect some pairs of vertices are called edges. Graphs have several features that make them appropriate for modeling wireless networks, and in particular their connectivity and interference. Graph-based interference models are particularly useful in the context of resource allocation or topology control [25, 26]. The connectivity of a wireless network can be modeled using a connectivity graph $G_{C}=\left(V, E_{C}\right)$, where $V$ is the set of vertices that represent the terminals of the network, and $E$ is the set of edges connecting any two vertices, which represent the communication links between the respective terminals. When the focus is on the interference, an interference graph $G_{\mathcal{I}}=\left(V_{\mathcal{I}}, E_{\mathcal{I}}\right)$ is used: if the intent is to model the interference between terminals, $V_{\mathcal{I}}$ is the set of terminals, while $E_{\mathcal{I}}$ is the set of edges, which model the interference between terminals; if the intent is to model the interference between links, $V_{\mathcal{I}}$ is the set of communication links, while $E_{\mathcal{I}}$ is the set of edges, which represent the interference between two links.

\subsection{Techniques to Analyze the Network Performance}

In the remainder of this dissertation a physical interference model is adopted, when the dynamics of the channel (e.g., fading and shadowing) and the random locations of the interferers are taken into account. In this case, $\gamma$ becomes a random variable and the cumulative distribution function (CDF) of the SINR is used as a metric for characterizing the performance of wireless networks. In the literature, there are six different techniques [27, 28], which are used to evaluate the CDF of the SINR once an interference model with a distance dependent power-law is adopted and interferers are drawn according to a specific point process: 
1. Rayleigh fading assumption: since it is not always possible to find a closed-form expression for the probability density function $(\underline{\mathrm{PDF}})$ of the aggregate interference [20] and in order to make the analysis tractable, it is common to assume Rayleigh fading. Under this assumption, it is not still possible to determine the interference statistic (the CDF of the aggregate interference), but the exact CDF of the SINR can be obtained, which has the form:

$$
F_{\gamma}(\beta)=P[\gamma \leq \beta]=1-\left.\exp \{-\mathcal{N} a \beta\} \mathcal{L}_{\mathcal{I}}(s)\right|_{s=a \beta}
$$

where $\mathcal{L}_{\mathcal{I}}(s)$ is the Laplace transform $(\overline{\mathrm{LT}})$ of the $\mathrm{PDF}$ of the aggregate interference $\mathcal{I}$ and $a$ is a constant. The key is to find the $\mathrm{LT}$ of the $\mathrm{PDF}$ of $\mathcal{I}$, which thanks to the probability generating functional ( $(\mathrm{PGFL})$ is easy to compute for many cases involving a $\mathrm{PPP}$ (e.g [14]) or other simple point processes such as a BPP (e.g [29]).

2. Dominant interferers assumption: when deterministic channel gains are assumed, this technique is used. Under this approach only a subset of interferers is considered, and in particular only the contribution from the interferers into a vulnerability region or $n$ nearest interferers are taken into account. Under a high path loss exponent (e.g., $\alpha=4$ ), the contribution from the further interferers is negligible and therefore this approach leads to a tight lower bound for the CDF of the SINR.

3. Approximated $\overline{P D F}$ of the aggregate interference: in this approach, simulations are used to empirically fit the $\mathrm{PDF}$ of $\mathcal{I}$ to a known distribution, such as a Gaussian or shifted log-normal.

4. Usage of Plancherel-Parseval theorem: an alternative approach to the previously ones is to use the Plancherel-Parseval theorem. This theorem states that if $f_{1}(t)$ and $f_{2}(t)$ are square integrable complex functions, then

$$
\int_{\mathbb{R}} f_{1}(t) f_{2}^{*}(t) \mathrm{d} t=\int_{\mathbb{R}} \mathcal{F}_{1}(\omega) \mathcal{F}_{2}^{*}(\omega) \mathrm{d} \omega
$$

where $f^{*}(t)$ is the conjugate of $f(t), \mathcal{F}_{1}(\omega)$ is the Fourier transform $(\overline{\mathrm{FT}})$ of $f_{1}(t)$, and $\mathcal{F}_{2}(\omega)$ is the $\mathrm{FT}$ of $f_{2}(t)$. Since the $\mathrm{FT}$ of a $\mathrm{PDF}$ is equivalent to the characteristic 
function of that $\mathrm{PDF}$, it is possible to state that $\mathcal{F}(\omega)=\left.\mathcal{L}(s)\right|_{s=i \omega}$. This last property allows the inverse $\mathrm{LT}$ to be replaced by an integral.

5. Laplace numerical inversion: this last technique makes use of numerical methods to invert the $\mathrm{LT}$ of the $\mathrm{PDF}$ of the aggregate interference.

6. Usage of Gil-Pelaez inversion theorem: in [28], the authors obtained exact and approximated closed-form expressions for the CDF of the SINR by inverting the characteristic function (CF) of the aggregate interference with the aid of the Gil-Pelaez inversion formula. This theorem states that

$$
F_{\mathcal{I}}(x)=\frac{1}{2}-\frac{1}{\pi} \int_{0}^{\infty} \frac{\operatorname{Im}\left[\exp (-i t x) \gamma_{\mathcal{I}}(t)\right]}{t} \mathrm{~d} t
$$

where $\gamma_{\mathcal{I}}(x)$ is the $\mathrm{CF}$ of the aggregated interference.

\subsection{Computational Complexity in Wireless Networks}

Wireless networks are designed to deliver information in real time, and therefore they are subject to very tight hard real-time timing and protocol constraints, which need to be regularly satisfied to guarantee their proper operation. One of the features that influences the reliability and the performance of a network is the computational complexity required to perform the processing of a signal. If, at a given time, a network requires more computational resources than those available, or it requires more time to complete a given operation than the one allowed, it is subject to an outage, and the information cannot be decoded. Therefore, based on the likelihood of this event, the performance of the network can be greatly impacted.

In modern wireless networks, the computational resources required over time mainly depends on the computational complexity of the channel decoder, which usually performs the most computational intense operations that increase as the system works closer to channel capacity. The computational resources are usually distributed in the network, and each device has its own computational capacity to perform channel decoding. However, novel architecture, such as Cloud-RAN [30] , use cloud-computing platform to perform centralized processing of multiple signals from a pool of $\mathrm{BS} s$, which enables the flexible usage of 
processing resources through virtualization [31, 32. Since these type of architectures are designed with the aim to reduce deployment costs throughout a parsimonious provisioning of the computational resources, they are particularly vulnerable to the detrimental effect of computational complexity. For this reason in the past years, an increasing interest has grown among researcher groups to better design these systems being aware of the computational penalties that characterize them.

\subsection{Scope, Outline and Contributions}

The focus of this dissertation is on the effect of interference and computational complexity in wireless communication systems. In particular, this work aims to build an alternative approach to the one listed in Sec. 1.2, which is able to discard some of common assumptions previously used and enables the more accurate modeling of both conventional and cooperative wireless systems and provide insights on how to optimize them. Focus is also given on estimating the data processing requirement for cellular networks that use centralized computing platforms, such as a Cloud-[RAN, to perform joint baseband processing on signals from a group of $\mathrm{BS}$ : The last part of this dissertation focuses then on $\mathrm{FH}$ systems and cooperative protocols and how to model and optimize them when accounting for the effect of interference.

The discussion starts in Chap. 2, which builds an alterative analytical framework to evaluate the outage probability of a network subject to fading and with the location of the interferers distributed according to a point process. In the literature $3,14,20,21,27,33,35$ a common assumption is to assume an infinite network, both by having an infinite area, and by having an infinite number of interferers. While this assumption simplifies the analysis, it is important to bear in mind that no wireless network is actually infinite. Furthermore, another drawback of the related literature is that the SINR variable combines the effects of fading and interferer location, and outage probabilities are computed with respect to both of these contributions. While certainly the long-term (c.f., ergodic) outage probability depends on both fading and location, it is important to realize that these mechanisms operate over time scales that are significantly different. Whereas the fading may change from one 
transmission to the next, the location of the active interferers will generally remain constant over the course of many transmissions. In Chap. 2 both assumptions are relaxed by using an approach which breaks the analysis into discrete steps that consider the fading and the random topology separately for a finite network. Using the aforementioned analytical framework, Chap. 2 continues by quantifying the effect of the spatial interference correlation in finite networks. In order to prove the wide applicability of the framework introduced in Chap. 2, Chap. 3 applies it to model and analyze the performance of direct-sequence code division multiplex-access (DS-CDMA) cellular uplinks. The model and the analysis, as will be more evident later, are very general and can handle a very large variety of MAC protocols and resource allocation policies.

Chap. 4 extends the analysis introduced in Chap. 2 in order to account for the case when the receiving device performs diversity combining. In this case, the analysis is applied to two cases of study: DS-CDMA downlink multi-cell cooperation (MCC) and an Long Term Evolution (LTE) transmission mode, called multicast-broadcast single-frequency network (MBSFN), which will be introduced in detail in this chapter. In both cases, an accurate model is build and a performance analysis is performed with the aim to emphasizing how cooperative communications should be used in these networks to maximize their benefits.

Chap. 5 focuses on the Cloud-RAN architecture [30]. While most of the recent research on Cloud-RAN has focused on the RAN functional split, the applicability of joint processing (e.g. [32]), system performance and implementation options (e.g. [31, 36]), and fronthaul requirements (e.g. [37]), this chapter provides a better understanding of the interplay between available computing resources and the required communications performance. In particular, Chap. 5 builds an analytical framework to estimate the required data processing resources under a given performance constraint, which allows to highlights the tradeoff between the throughput and the computational complexity that characterizes these type of networks, that is investigated in more details in Chap. 6. Chap. 6 provides insights on how the communications performance are effected by the computational resources available. Furthermore, it provides how to optimally allocate the user rate under the assumption of limited computational resources in a centralized cloud platform and presents three computationally aware schedulers. 
Chap. 7 focuses then on FH systems in general, and in particular, considers ad hoc networks that use a non-coherent continuous-phase frequency-shift keying (CPFSK) modulation. This modulation format depends on the modulation index, the number of frequency channels in which the overall bandwidth is divided and the fractional in-band power, which is the fraction of the signal power that lies within the band of each frequency channel. These parameters are often chosen arbitrarily. However, in this chapter it is shown under two different rate-allocation policies that optimally selecting them together with the rate, it is possible to achieve a significant improvement in the performance of the system.

Chap. 8 introduces a new methodology to model and analyze the performance of multihop routing protocols in ad hoc networks which builds on the analytical framework introduced in Chap. 2. This chapter accounts for the effect of the interference and their spatial correlation and evaluates the performance of two representative geographic routing protocols in order to gain perspective about their advantages and disadvantages.

Chap. 9 introduces an efficient method to stochastically model and study the performance of cooperative multi-hop networks when accounting for CCI originated by multiple packets that simultaneously propagate along the network. The dynamics of how a cooperative protocol evolves over time is described through an absorbing Markov chain, which uses the analysis developed in Chap. 4 to evaluate the transmission probability of each relay. This methodology differs from the ones existing in the literature, since the end-to-end probability of success of a cooperative network is not evaluated by starting from a single set of channel realizations (as it is usually done) and then averaging over them, but through the channel outage framework developed along this dissertation. As already stated, this framework provides the channel outage probability already averaged over the fading and in this context it drastically reduces the computational effort to model and analyze a cooperative multi-hop network. As an example of its applicability, this framework is used in this chapter to analyze the performance of a Barrage relay network (BRN) and perform an optimization of such a system over a set of parameters, which includes the code rate, and the number of relays used.

Finally, this dissertation concludes in Chap. 10, which provides some suggestions for future work. 


\section{Chapter 2}

\section{Channel Outage Analysis in Wireless Networks}

Outage probability is a useful and popular metric for characterizing the performance of wireless networks. Its average over the locations of the interferers, which is called the spatial average, is able to capture in a single quantity the dynamics of both the channel (e.g., fading and shadowing) and the random locations of the sources of interference. After introducing the network model, this chapter describes a new analytical framework for computing the spatially averaged outage probability of a network with interferers located according to a point process and signals subject to fading. The analysis begins by fixing the network geometry, and the outage probability in the presence of fading is computed conditioned on the network geometry, which describes the location of the interferers and the shadowing of the interfering signals. The spatially averaged outage probability is then obtained by averaging the conditional outage probability with respect to the network geometry for several different distributions for the interferer locations.

While a significant amount of research has been devoted to modeling and quantifying the effect of interference in wireless networks, only recently there has been some work 3844 that focuses on the analysis and quantification of spatial interference correlation. Interference correlation arises because a common set of potential interferers are observed through similarly attenuated channels, even if the fading is uncorrelated in space and time, and it produces a significant diversity loss. Using the aforementioned analytical framework, the 
chapter proceeds by analytically characterizing this effect, and semi-closed form expressions are derived for some special cases. The last part of the chapter presents numerical results to highlight the correctness of the analysis and draw some conclusions.

\section{$2.1 \quad$ Network Model}

Consider a network comprising $M+2$ devices that include a reference receiver $Y_{j}$, a source or reference transmitter $X_{0}$, and $M$ interfering devices $X_{1}, \ldots, X_{M}$. The interferers are located within an arbitrary region $\mathcal{A}$, which has area $|\mathcal{A}|$. The number of interferers within $\mathcal{A}$ could be fixed (as in a $\overline{\mathrm{BPP}}$ ) or random (as in a $\overline{\mathrm{PPP}}$ ). Let $r_{i, j}$ denote the distance from $X_{i}$ and the receiver $Y_{j}$, and let $\boldsymbol{r}=\left\{r_{1, j}, \ldots, r_{M, j}\right\}$ represent the set of distances to the interferers, which corresponds to a specific network topology.

While the interfering devices can be located in any arbitrary region, it is assumed they are located within the arbitrary region $\mathcal{A}$, but outside a circular region with radius $r_{\text {in }}$, which is centered at the receiver. A nonzero $r_{\text {in }}$ may be used to model an exclusion or guard zone 45,46 . As mentioned in the previous chapter, an exclusion zone is a region where no other transmitter is allowed to be located. On the other hand, another transmitter might be within a guard zone, but this transmitter is suppressed by the MAC protocol. A guard zone can be realized by having the receiver send a short clear-to-send (CTS) packet in response to a request-to-send ( $\overline{\mathrm{RTS}})$ packet sent by the transmitter. Under a carrier sense multiple access (CSMA) protocol, devices within distance $r_{\text {in }}$ from the receiver that overhear the CTS will suppress their transmissions.

Device $X_{i}$ transmits a signal whose average received power in the absence of fading and shadowing is $P_{i}$ at a reference distance $d_{0}$. At the reference receiver, $X_{i}$ 's power is

$$
\rho_{i, j}=P_{i} g_{i, j} 10^{\xi_{i, j} / 10} f(r)
$$

where $g_{i, j}$ is the power gain due to fading, $\xi_{i, j}$ is a shadowing coefficient, and $f(r)$ is a path loss function. Each $g_{i, j}=a_{i, j}^{2}$, where $a_{i, j}$ has a Nakagami distribution with parameter $m_{i, j}$, and $\mathbb{E}\left[g_{i, j}\right]=1$. In Rayleigh fading, $m_{i, j}=1$ and $g_{i, j}$ is exponential. In the presence of log-normal shadowing, the $\left\{\xi_{i, j}\right\}$ are independent and identically distributed zero-mean 
Gaussian with standard deviation $\sigma_{\mathrm{s}} \mathrm{dB}$. In the absence of shadowing, $\xi_{i, j}=0$. For $r \geq d_{0}$, the path loss function is expressed as the attenuation power law

$$
f(r)=\left(\frac{r}{d_{0}}\right)^{-\alpha}
$$

where $\alpha>2$ is the attenuation power-law exponent, and $d_{0}$ is sufficiently large that the signals are in the far field.

The instantaneous SINR at the reference receiver $Y_{j}$ is

$$
\gamma_{j}=\frac{\rho_{0, j}}{\mathcal{N}+\sum_{i=1}^{M} I_{i} \rho_{i, j}}
$$

where $\mathcal{N}$ is the noise power and $I_{i}$ is a Bernoulli variable with $\mathbb{P}\left[I_{i}=1\right]=p_{i}$, where the activity probability $p_{i}$ can be used to model voice-activity factors or controlled silence.

Substituting (2.1) and 2.2) into (2.3), the SINR is

$$
\gamma_{j}=\frac{g_{0, j} \Omega_{0, j}}{\mathrm{SNR}^{-1}+\sum_{i=1}^{M} I_{i} g_{i, j} \Omega_{i, j}}
$$

where SNR $=d_{0}^{\alpha} P_{0} / \mathcal{N}$ is the signal-to-noise ratio (SNR) when the transmitter is at unit distance and fading and shadowing are absent, and

$$
\Omega_{i, j}= \begin{cases}10^{\xi_{0, j} / 10} r_{0, j}^{-\alpha} & i=0 \\ \frac{P_{i}}{P_{0}} 10^{\xi_{i, j} / 10} r_{i, j}^{-\alpha} & i \geq 1\end{cases}
$$

is the normalized received power due to $X_{i}$.

\subsection{Conditional Outage Probability}

Let $\beta$ denote the minimum SINR required for reliable reception and $\Omega=\left\{\Omega_{0, j}, \ldots, \Omega_{M, j}\right\}$ represent the set of normalized received powers. An outage occurs when the SINR falls below $\beta$. When conditioned on $\Omega$, the outage probability is

$$
\epsilon_{j}(\Omega)=\mathbb{P}\left[\gamma_{j} \leq \beta \mid \Omega\right]
$$


Because it is conditioned on $\Omega$, the outage probability depends on the locations of the devices and the shadowing factors, which have dynamics over timescales that are much slower than the fading.

Defining a variable

$$
\mathrm{Z}_{j}=\mathrm{S}_{j}-\sum_{i=1}^{M} \mathrm{Y}_{i, j}
$$

where $\mathbf{S}_{j}=\beta^{-1} g_{0, j} \Omega_{0, j}$ and $\mathbf{Y}_{i, j}=I_{i} g_{i, j} \Omega_{i, j}$, the outage probability, conditioned on $\boldsymbol{\Omega}$, may be expressed as

$$
\epsilon_{j}(\Omega)=\mathbb{P}\left[\mathrm{Z}_{j} \leq \mathrm{SNR}^{-1} \mid \Omega\right]=F_{\mathrm{Z}_{j}}\left(\mathrm{SNR}^{-1} \mid \Omega\right)
$$

which is the $\mathrm{CDF}$ of $\mathrm{Z}_{j}$ conditioned on $\Omega$ and evaluated at $\mathrm{SNR}^{-1}$.

Theorem 2.2.1 The conditional outage probability is

$$
\begin{aligned}
\epsilon_{j}(\Omega)= & 1-\exp \left(-\frac{\beta m_{0, j}}{\Omega_{0, j} \mathrm{SNR}}\right) \sum_{\mu=0}^{m_{0, j}-1}\left(\frac{\beta m_{0, j}}{\Omega_{0, j} \mathrm{SNR}}\right)^{\mu} \sum_{t=0}^{\mu} \frac{\mathrm{SNR}^{t}}{(\mu-t) !} \sum_{\substack{\ell_{i} \geq 0 \\
\sum_{i=1}^{M} \ell_{i}=t}} \\
& \prod_{i=1}^{M}\left[\left(1-p_{i}\right) \delta_{\ell_{i}}+\frac{p_{i} \Gamma\left(\ell_{i}+m_{i, j}\right)}{\ell_{i} ! \Gamma\left(m_{i, j}\right)}\left(\frac{\Omega_{i, j}}{m_{i, j}}\right)^{\ell_{i}}\left(\frac{\beta m_{0, j} \Omega_{i, j}}{m_{i, j} \Omega_{0, j}}+1\right)^{-\left(m_{i, j}+\ell_{i}\right)}\right] .
\end{aligned}
$$

Proof: The details of the derivation are given in Appendix A.

When all transmissions are subject to Rayleigh fading, the $\left\{g_{i, j}\right\}$ are i.i.d. unit-mean exponential random variables, and by simply substituting $m_{0, j}=m_{i, j}=1$ (2.9), the outage probability conditioned on $\Omega$ is

$$
\epsilon_{j}(\Omega)=1-\exp \left(-\frac{\beta}{\Omega_{0, j} \mathrm{SNR}}\right) \prod_{i=1}^{M}\left(\frac{\Omega_{0, j}+\beta\left(1-p_{i}\right) \Omega_{i, j}}{\Omega_{0, j}+\beta \Omega_{i, j}}\right) .
$$

The above expression is obtained in [47] working iteratively. Alternatively, when the distances are normalized such that the reference transmitter is at unit distance from the receiver, shadowing is neglected and under the assumption that $p_{i}=p$ and $P_{i}=P_{0}$ for all $i \in\{1, \ldots, M\}, 2.10$ can be found from [20] by substituting that reference's (2.3) into its (1.4). 


\subsection{Spatially Averaged Outage Probability}

The spatially averaged outage probability is found by taking the expectation of $\epsilon(\boldsymbol{\Omega})$ with respect to the network geometry and shadowing factors. Let $\epsilon_{j}[M]$ denote the spatially averaged outage probability of a network with a fixed number of interferers, which is found by taking the expectation of $\epsilon_{j}(\Omega)$ with respect to $\Omega$ under the condition that there are $M$ interferers; i.e.,

$$
\epsilon_{j}[M]=\mathbb{E}_{\boldsymbol{\Omega}}\left[\epsilon_{j}(\boldsymbol{\Omega}) \mid M\right]=\int \epsilon_{j}(\boldsymbol{\Omega}) f_{\boldsymbol{\Omega}}(\boldsymbol{\Omega} \mid M) \mathrm{d} \boldsymbol{\Omega}
$$

where $f_{\Omega}(\Omega \mid M)$ is the PDF of $\Omega$ when there are $M$ interferers.

When the number of interferers is random, then the overall spatially averaged outage probability, which we denote $\epsilon_{j}$, can be found by taking the expectation of $\epsilon_{j}[M]$ with respect to the number of interferers in the region. In particular,

$$
\epsilon_{j}=\mathbb{E}_{M}\left[\epsilon_{j}[M]\right]=\sum_{m=0}^{\infty} p_{M}[m] \epsilon_{j}[m]
$$

where $p_{M}[m]$ is the probability mass function ( $(\overline{\mathrm{PMF}})$ of $M$.

\subsubsection{Binomial Point Processes}

Assume that the $M$ interferers are independently and uniformly distributed (i.u.d.) over $\mathcal{A}$. Thus, the interferers are drawn from a $\overline{\mathrm{BPP}}$ of intensity $\lambda=M /|\mathcal{A}|[21]$. Because the $\left\{\Omega_{i, j}\right\}$ are independent,

$$
f_{\Omega}(\Omega \mid M)=\prod_{i=1}^{M} f_{\Omega}\left(\Omega_{i, j}\right)
$$

where $f_{\Omega}\left(\Omega_{i, j}\right)$ is the $\mathrm{PDF}$ of $\Omega_{i, j}$.

Substituting (2.9) and (2.13) into (2.11) and using the fact that the $\left\{\Omega_{i, j}\right\}$ are identically distributed, the spatially averaged outage probability can be found as follows

$$
\begin{aligned}
\epsilon_{j}[M]= & 1-\exp \left(-\frac{\beta m_{0, j}}{\Omega_{0, j} \mathrm{SNR}}\right) \sum_{\mu=0}^{m_{0, j}-1}\left(\frac{\beta m_{0, j}}{\Omega_{0, j} \mathrm{SNR}}\right)^{\mu} \sum_{t=0}^{\mu} \frac{\mathrm{SNR}^{t}}{(\mu-t) !} \\
& \sum_{\substack{\ell_{i} \geq 0 \\
\sum_{i=1}^{M} \ell_{i}=t}} \prod_{i=1}^{M}\left[\left(1-p_{i}\right) \delta_{\ell_{i}}+\frac{p_{i} \Gamma\left(\ell_{i}+m_{i, j}\right)}{\ell_{i} ! \Gamma\left(m_{i, j}\right)} \digamma\left(i, \ell_{i}\right)\right]
\end{aligned}
$$


where

$$
\digamma\left(i, \ell_{i}\right)=\int\left(\frac{\Omega_{i, j}}{m_{i, j}}\right)^{\ell_{i}}\left(\frac{\beta m_{0, j} \Omega_{i, j}}{m_{i, j} \Omega_{0, j}}+1\right)^{-\left(m_{i, j}+\ell_{i}\right)} f_{\Omega}\left(\Omega_{i}, j\right) \mathrm{d} \Omega_{i, j} .
$$

If an unshadowed scenario is assumed, the normalized received power is equal to $\Omega_{0, j}=$ $r_{0, j}^{-\alpha}$ for the reference transmitter and $\Omega_{i, j}=\frac{P_{i}}{P_{0}} r_{i, j}^{-\alpha}$ for each of the $i^{\text {th }}$ interferers. Since the only source of randomness in $\left\{\Omega_{i, j}\right\}$ is $\left\{r_{i, j}\right\}$, which is the set of distances between the reference receiver and the interferers, 2.15) can be simplified as follows:

$$
\digamma\left(i, \ell_{i}\right)=\mathcal{P}\left(i, \ell_{i}\right) \int r^{m_{i, j}^{\alpha}}\left[\mathcal{H}\left(i, \ell_{i}\right) r^{\alpha}+1\right]^{-\left(m_{i, j}+\ell_{i}\right)} f_{r}(r) \mathrm{d} r
$$

where $\mathcal{H}\left(i, \ell_{i}\right)=\frac{m_{i, j} P_{0}}{\beta m_{0, j} P_{i, j} r_{0, j}^{\alpha}}, \mathcal{P}\left(i, \ell_{i}\right)=\mathcal{H}\left(i, \ell_{i}\right)^{m_{i, j}} \frac{1}{\left(r_{0, j}^{\alpha} m_{0, j} \beta\right)^{\ell_{i}}}$ and $f_{r}(r)$ is the PDF of the identically distributed $\left\{r_{i, j}\right\}$.

\section{L-sided Regular Polygon Network}

Assume that the reference receiver is located at the origin and that $\mathcal{A}$ is an L-sided regular polygon inscribed in a circle of radius $r_{\text {out }}$. The polygon is centered at the origin, and a circular exclusion zone of radius $r_{\text {in }}$ is removed from the center of the polygon to ensure that all interferers are at least distance $r_{\text {in }}$ away. The area of this region is $|\mathcal{A}|=$ $\frac{1}{2} L r_{\text {out }}^{2} \sin \left(\frac{2 \pi}{L}\right)-\pi r_{\text {in }}^{2}$. Let $r_{\mathrm{c}}=r_{\text {out }} \sin (\pi(L-2) /(2 L))$ be the distance from the origin to any corner of the polygon. From [48], the identically distributed $\left\{r_{i, j}\right\}$ have the PDF

$$
f_{r}(r)= \begin{cases}\frac{2 \pi r}{|\mathcal{A}|} & \text { for } r_{\text {in }} \leq r \leq r_{\mathrm{c}} \\ \frac{2 \pi r}{|\mathcal{A}|}-\frac{2 L r}{|\mathcal{A}|} \arccos \left(\frac{r_{\mathrm{c}}}{r}\right) & \text { for } r_{\mathrm{c}} \leq r \leq r_{\text {out }}\end{cases}
$$

and zero elsewhere.

When (2.17) is substituted into 2.16), the integral is

$$
\int r^{m_{i, j} \alpha}\left[\mathcal{H}\left(i, \ell_{i}\right) r^{\alpha}+1\right]^{-\left(m_{i, j}+\ell_{i}\right)} f_{r}(r) \mathrm{d} r=T_{1}\left(i, \ell_{i}\right)-T_{2}\left(i, \ell_{i}\right)
$$

where

$$
\begin{aligned}
T_{1}\left(i, \ell_{i}\right) & =\frac{2 \pi}{|\mathcal{A}|} \int_{r_{\text {in }}}^{r_{\text {out }}} r^{m_{i, j} \alpha+1}\left[\mathcal{H}\left(i, \ell_{i}\right) r^{\alpha}+1\right]^{-\left(m_{i, j}+\ell_{i}\right)} \mathrm{d} r \\
T_{2}\left(i, \ell_{i}\right) & =\frac{2 L}{|\mathcal{A}|} \int_{r_{\mathrm{c}}}^{r_{\text {out }}} r^{m_{i, j} \alpha+1}\left[\mathcal{H}\left(i, \ell_{i}\right) r^{\alpha}+1\right]^{-\left(m_{i, j}+\ell_{i}\right)} \arccos \left(\frac{r_{\mathrm{c}}}{r}\right) \mathrm{d} r .
\end{aligned}
$$


The first integral is

$$
T_{1}\left(i, \ell_{i}\right)=\frac{2 \pi}{|\mathcal{A}|}\left[\Psi_{1}\left(i, \ell_{i}, r_{\text {out }}\right)-\Psi_{1}\left(i, \ell_{i}, r_{\text {in }}\right)\right]
$$

where

$$
\Psi_{k}\left(i, \ell_{i}, y\right)=\int_{0}^{y} r^{m_{i, j} \alpha+k}\left[\mathcal{H}\left(i, \ell_{i}\right) r^{\alpha}+1\right]^{-\left(m_{i, j}+\ell_{i}\right)} \mathrm{d} r
$$

By performing the change of variable $r=y \nu^{\frac{1}{\alpha}}$,

$$
\begin{aligned}
\Psi_{k}\left(i, \ell_{i}, y\right) & =\frac{y^{m_{i, j} \alpha+k+1}}{\alpha} \int_{0}^{1} \nu^{\left(m_{i, j} \alpha+k+1-\alpha\right) / \alpha}\left[\mathcal{H}\left(i, \ell_{i}\right) y^{\alpha} \nu+1\right]^{-\left(m_{i, j}+\ell_{i}\right)} \mathrm{d} \nu \\
& =\left(\frac{y^{m_{i, j} \alpha+k+1}}{m_{i, j} \alpha+k+1}\right){ }_{2} F_{1}\left(\left[m_{i, j}+\ell_{i}, \frac{m_{i, j} \alpha+k+1}{\alpha}\right] ; \frac{m_{i, j} \alpha+k+1}{\alpha}+1 ;-\mathcal{H}\left(i, \ell_{i}\right) y^{\alpha}\right)(2.2
\end{aligned}
$$

where ${ }_{2} F_{1}$ is the Gauss hypergeometric function,

$$
{ }_{2} F_{1}([a, b] ; c ; x)=\frac{\Gamma(c)}{\Gamma(b) \Gamma(c-b)} \int_{0}^{1} \nu^{b-1}(1-\nu)^{c-b-1}(1-x \nu)^{-a} \mathrm{~d} \nu
$$

and since $c=b+1, \Gamma(c) /(\Gamma(b) \Gamma(c-b))=b$. As the hypergeometric function only converges for $|x|<1$, it follows that convergence requires $\frac{1}{\mathcal{H}_{i}}>y$, which is satisfied by the following set of sufficient conditions: (1) $\beta>1$ (or $0 \mathrm{~dB}$ ), which is true for most modulation/coding schemes of interest;(2) $P_{i} \leq P_{0}$, which is always true under all power control policies; (3) $y \leq 1$, which is true when the network is normalized to $r_{\text {out }}=1$; and (4) $m_{0, j} \geq m_{i, j}$, which is true when the reference transmitter is more line-of-sight (을 $)$ than the interferers. Note also that (2.24) does not converge when $c$ is a non-positive integer. Furthermore, it is important to note that while the hypergeometric function is itself an integral, it is widely known and is implemented as a single function call in most mathematical programming languages, including Matlab.

The above analysis agrees with [49], which does not include an exclusion zone $\left(r_{\text {in }}=0\right)$, and uses numerical integration to evaluate (2.20). Alternatively, the second integral can be found by substituting the Taylor series expansion of arccos given by [50]

$$
\arccos (x)=\frac{\pi}{2}-\sum_{n=0}^{\infty} \frac{(2 n) ! x^{2 n+1}}{4^{n}(n !)^{2}(2 n+1)}
$$

into 2.20$)$, resulting in

$$
T_{2}\left(i, \ell_{i}\right)=\frac{2 L}{|\mathcal{A}|}\left[\mathcal{K}\left(i, \ell_{i}, r_{\text {out }}\right)-\mathcal{K}\left(i, \ell_{i}, r_{\mathrm{c}}\right)\right]
$$


where

$$
\mathcal{K}\left(i, \ell_{i}, y\right)=\frac{\pi}{2} \Psi_{1}\left(i, \ell_{i}, y\right)-\sum_{n=0}^{\infty} \frac{(2 n) ! r_{\mathrm{c}}^{2 n+1}}{4^{n}(n !)^{2}(2 n+1)} \Psi_{-2 n}\left(i, \ell_{i}, y\right)
$$

By substituting (2.18) into (2.16) with $T_{1}\left(i, \ell_{i}\right)$ given by (2.21) and $T_{2}\left(i, \ell_{i}\right)$ given by (2.26), it yields

$$
\digamma\left(i, \ell_{i}\right)=\frac{2 \mathcal{P}\left(i, \ell_{i}\right)}{|\mathcal{A}|}\left[\pi \Psi_{1}\left(i, \ell_{i}, r_{\text {out }}\right)+L \mathcal{K}\left(i, \ell_{i}, r_{\mathrm{c}}\right)-\pi \Psi_{1}\left(i, \ell_{i}, r_{\text {in }}\right)-L \mathcal{K}\left(i, \ell_{i}, r_{\text {out }}\right)\right]
$$

Finally by substituting $(2.28)$ into 2.14$)$ it is possible to obtain a closed form expression for the spatially averaged outage probability.

If all transmissions are subject to Rayleigh fading $\left(m_{0, j}=m_{i, j}=1\right)$, and under the assumption that $p_{i}=p$ and $P_{i}=P_{0}$ for all $i \in\{1, \ldots, M\}$, the spatially averaged outage probability is 51

$$
\epsilon[M]=1-\exp \left(-\frac{\beta r_{0, j}^{\alpha}}{\mathrm{SNR}}\right)\left[1+\frac{p}{|\mathcal{A}|} \Theta\left(r_{\text {in }}, r_{\mathrm{c}}, r_{\text {out }}\right)\right]^{M}
$$

where

$$
\begin{aligned}
\Theta(x, y, z) & =\pi[\Phi(z)-\Phi(x)]-\frac{2 L}{\beta r_{0_{j}}^{\alpha}}[K(z)-K(y)]-\mathcal{C}(z) \\
\Phi(x) & =\frac{2}{\beta r_{0_{j}}^{\alpha}} \Psi_{1}(x)-x^{2} \\
\mathcal{C}(x) & =x^{2}\left[\frac{1}{2} L \sin \left(\frac{2 \pi}{L}\right)-\pi\right] \\
\Psi_{k}(x) & =\Psi_{k}\left(i, \ell_{i}, x\right) \\
\mathcal{K}(x) & =\mathcal{K}\left(i, \ell_{i}, x\right) .
\end{aligned}
$$

\section{Annular Network}

Assume that the reference receiver is located at the origin and that the interferers are in an annular region with inner radius $r_{\text {in }}$ and outer radius $r_{\text {out }}$. Clearly, this shape is the limiting case of the polygon as $L \rightarrow \infty$. In this case, $r_{\mathrm{c}} \rightarrow r_{\text {out }}$, and substituting 2.28 into 
(2.14),

$$
\begin{aligned}
\epsilon_{j}[M]= & 1-\exp \left(-\frac{\beta m_{0, j}}{\Omega_{0, j} \mathrm{SNR}}\right) \sum_{\mu=0}^{m_{0, j}-1}\left(\frac{\beta m_{0, j}}{\Omega_{0, j} \mathrm{SNR}}\right)^{\mu} \sum_{t=0}^{\mu} \frac{\mathrm{SNR}^{t}}{(\mu-t) !} \sum_{\substack{\ell_{i} \geq 0 \\
\sum_{i=1}^{M} \ell_{i}=t}} \\
& \prod_{i=1}^{M}\left\{\left(1-p_{i}\right) \delta_{\ell_{i}}+\frac{2 \pi p_{i} \Gamma\left(\ell_{i}+m_{i, j}\right) \mathcal{P}\left(i, \ell_{i}\right)}{\ell_{i} ! \Gamma\left(m_{i, j}\right)|\mathcal{A}|}\left[\Psi_{1}\left(i, \ell_{i}, r_{\text {out }}\right)-\Psi_{1}\left(i, \ell_{i}, r_{\text {in }}\right)\right]\right\}
\end{aligned}
$$

where $|\mathcal{A}|=\pi\left(r_{\text {out }}^{2}-r_{\text {in }}^{2}\right)$.

If all transmissions are subject to Rayleigh fading and under the assumption that $p_{i}=p$ and $P_{i}=P_{0}$ for all $i \in\{1, \ldots, M\}$, since $r_{\mathrm{c}} \rightarrow r_{\text {out }}$ and $\mathcal{C}\left(r_{\text {out }}\right) \rightarrow 0,(2.29)$ becomes [51

$$
\epsilon_{j}[M]=1-e^{-\beta r_{0_{j}}^{\alpha} / \mathrm{SNR}}\left(1+\frac{p \pi}{|\mathcal{A}|}\left[\Phi\left(r_{\text {out }}\right)-\Phi\left(r_{\text {in }}\right)\right]\right)^{M} .
$$

To compute the spatially averaged outage probability in the presence of shadowing, the PDFs $f_{\Omega_{0, j}}\left(\omega_{0, j}\right), \ldots, f_{\Omega_{M, j}}\left(\omega_{M, j}\right)$ used in 2.14$)$ need to be derived to account for the shadowing distribution. First consider the normalized power of the desired signal from the reference transmitter $X_{0}$. Because the distance $r_{0, j}$ between the reference transmitter and receiver is deterministic, $\Omega_{0, j}=10^{\xi_{0, j} / 10} r_{0, j}^{-\alpha}$ is a log-normal variable with $\overline{\mathrm{PDF}}$

$$
f_{\Omega_{0, j}}(\omega)=\frac{10\left(2 \pi \sigma_{\mathrm{s}}^{2}\right)^{-\frac{1}{2}}}{\ln (10) \omega} \exp \left\{-\frac{10^{2} \log _{10}^{2}\left(r_{0}^{\alpha} \omega\right)}{2 \sigma_{\mathrm{s}}^{2}}\right\}
$$

for $0 \leq \omega \leq \infty$, and zero elsewhere.

For $i \geq 1$, corresponding to the devices drawn from the $\mathrm{BPP}$, the $\mathrm{PDF}$ is

$$
f_{\Omega_{i, j}}(\omega)=\omega^{-\frac{2+\alpha}{\alpha}} \frac{\left[\zeta\left(c_{i} \omega r_{\text {out }}^{\alpha}\right)-\zeta\left(c_{i} \omega r_{\text {in }}^{\alpha}\right)\right]}{\alpha c_{i}^{-2 / \alpha}\left(r_{\text {out }}^{2}-r_{\text {in }}^{2}\right)}
$$

for $0 \leq \omega \leq \infty$, and zero elsewhere, where $c_{i}=\left(P_{0} / P_{i}\right)$ and

$$
\zeta(z)=\operatorname{erf}\left(\frac{\sigma_{\mathrm{s}}^{2} \ln ^{2}(10)-50 \alpha \ln (z)}{5 \sqrt{2} \alpha \sigma_{\mathrm{s}} \ln (10)}\right) e^{\frac{\sigma_{\mathrm{s}}^{2} \ln ^{2}(10)}{50 \alpha^{2}}} .
$$

By substituting 2.9) and 2.13) into 2.11) , using (2.37) and 2.38) and after few algebraic manipulations, the spatially averaged outage probability is found to be

$$
\begin{aligned}
\epsilon_{j}[M]= & 1-\sum_{s=0}^{m_{0, j}-1} \sum_{t=0}^{s} \frac{\mathrm{SNR}^{t}}{(s-t) !} \sum_{\substack{\ell_{i} \geq 0 \\
\sum_{i=0}^{M} \ell_{i}=t}}\left\{\int_{0}^{\infty} \exp \left\{-\frac{\beta m_{0, j}}{y \mathrm{SNR}}\right\}\left(\frac{\beta m_{0, j}}{y \mathrm{SNR}}\right)^{s}\right. \\
& \left.\times \prod_{i=1}^{M}\left[\left(1-p_{i}\right) \delta_{\ell_{i}}+\int_{0}^{\infty} \kappa\left(\omega, \frac{\beta m_{0, j}}{y}\right) \varphi(\omega) d \omega\right] f_{\Omega_{0, j}}(y) \mathrm{d} y\right\}
\end{aligned}
$$


where

$$
\begin{aligned}
\varphi(\omega) & =\zeta\left(c_{i} \omega r_{\text {out }}^{\alpha}\right)-\zeta\left(c_{i} \omega r_{\text {in }}^{\alpha}\right) \\
\kappa(x, t) & =\frac{p_{i} \Gamma\left(\ell_{i}+m_{i, j}\right) x^{-\left(\frac{2+\alpha}{\alpha}\right)}\left(\frac{x}{m_{i, j}}\right)^{\ell_{i}}}{\alpha c_{i}^{2 / \alpha}\left(r_{\text {out }}^{2}-r_{\text {in }}^{2}\right)\left(\ell_{i} !\right) \Gamma\left(m_{i, j}\right)\left(\frac{x t}{m_{i, j}}+1\right)^{\left(m_{i, j}+\ell_{i}\right)}} .
\end{aligned}
$$

In order to compute the outage probability, the integral inside the product in 2.40 can be evaluated numerically by Simpson's method, which provides a good tradeoff between accuracy and speed, while the second integral can be evaluated through Monte Carlo integration.

\section{Arbitrary Area}

Assume that the reference receiver is located at the origin and that the interferers are in an arbitrarily shaped area. In this case, the integral in $(2.16)$ cannot generally be evaluated in closed form for an unshadowed scenario and the analysis doesn't reduce to closed form expression for a shadowed scenario as well. However, for the unshadowed scenario the integral in (2.16) is just a one-dimensional integral and as such, it can easily be evaluated numerically for each term of the multinomial summation in (2.14).

In Rayleigh fading, for an unshadowed scenario and under the assumption that $p_{i}=p$ and $P_{i}=P_{0}$ for all $i \in\{1, \ldots, M\}$, the spatially averaged outage probability can be found by substituting (2.10) and 2.13) into (2.11) and using the fact that the $\left\{r_{i, j}\right\}$ are identically distributed:

$$
\epsilon_{j}[M]=1-\exp \left(-\frac{\beta r_{0, j}^{\alpha}}{\mathrm{SNR}}\right)\left[1-p+p \int f_{r}(r) \frac{r^{\alpha}}{\beta r_{0_{j}}^{\alpha}+r^{\alpha}} d r\right]^{M}
$$

It follows that in this case computing the outage probability for a $\mathrm{BPP}$ boils down to evaluating a single one-dimensional integral.

In particular, the integral can be expressed as

$$
\int f_{r}(r) \frac{r^{\alpha}}{\beta r_{0_{j}}^{\alpha}+r^{\alpha}} d r=\mathbb{E}_{r}\left[\frac{r^{\alpha}}{\beta r_{0_{j}}^{\alpha}+r^{\alpha}}\right] .
$$

The right side of (2.44) suggests a simple numerical approach to solving the integral: Draw a large number of points $\left\{r_{i, j}\right\}$ distributed according to $f_{r}(r)$, and for each one of the points 
evaluate the function $g(r)=\left(1+\beta r_{0_{j}}^{\alpha} r^{-\alpha}\right)^{-1}$. The integral is then well approximated by the average of the $\left\{g\left(r_{i, j}\right)\right\}$. This is an alternative to [49], which requires $f_{r}(r)$ to be determined and substituted into the integral before it is numerically integrated. When the process is homogeneous, the $\left\{r_{i, j}\right\}$ can be selected by placing the shape inside a box, randomly selecting a point with uniform probability in the box and if the point falls within the shape, including it in the set of $\left\{r_{i, j}\right\}$, then repeating the process until $\left\{r_{i, j}\right\}$ is sufficiently large. This approach is a Monte Carlo simulation, but it is a simple simulation of the location of a single interferer. Importantly, it is not a simulation that requires the fading coefficients to be realized, and it does not require all $M$ interferers to be placed. Alternatively, the $\left\{r_{i, j}\right\}$ could be selected by overlaying the shape with a fine grid of equally spaced points.

\subsubsection{Poisson Point Processes}

Suppose that the interferers are drawn from a $\mathrm{PPP}$ with intensity $\lambda$ on the plane $\mathbb{R}^{2}$. Let $\epsilon_{j}(\mathcal{A})$ denote the outage probability considering only those interferers located within region $\mathcal{A}$. The number of interferers $M$ within region $\mathcal{A}$ is Poisson with mean $\mathbb{E}[M]=\lambda|\mathcal{A}|$. It follows that the $\mathrm{PMF}$ of the number of interferers within $\mathcal{A}$ is given by 21

$$
p_{M}[m]=\frac{(\lambda|\mathcal{A}|)^{m}}{m !} \exp (-\lambda|\mathcal{A}|), \text { for } m \geq 0 .
$$

The outage probability can then be found for any arbitrary $\mathcal{A}$ and for both a shadowed

and an unshadowed scenario by substituting (2.45) and the appropriate $\epsilon_{j}[m]$ into 2.12 . For the general case that $m_{0, j}$ may be any positive integer, the computation of 2.12 requires the summation to be truncated. However, in the specific case of Rayleigh fading $\left(m_{i, j}=1\right.$ for all $i$ ) and no shadowing, and under the assumption that the reference receiver is located at the center of the network, the summation may be found in closed form.

\section{L-sided Regular Polygon Network}

Assuming Rayleigh fading and that both $P_{i}=P_{0}$ and $p_{i}=p$ are common for all $i$, substituting 2.45 and 2.29 into 2.12) gives

$$
\epsilon_{j}(\mathcal{A})=1-\exp \left(-\lambda|\mathcal{A}|-\frac{\beta r_{0, j}^{\alpha}}{\mathrm{SNR}}\right) \sum_{m=0}^{\infty} \frac{1}{m !}\left[\lambda|\mathcal{A}|+\lambda p \Theta\left(r_{\text {in }}, r_{\mathrm{c}}, r_{\text {out }}\right)\right]^{m} .
$$


By using the power-series representation of the exponential function given by 50

$$
\sum_{m=0}^{\infty} \frac{a^{m}}{m !}\left(1-c \cdot b^{m}\right)=e^{a}-c \cdot e^{a \cdot b}
$$

into $(2.46)$, the spatially averaged outage probability is found to be

$$
\epsilon_{j}(\mathcal{A})=1-\exp \left\{-\frac{\beta r_{0}^{\alpha}}{\mathrm{SNR}}+\lambda p \Theta\left(r_{\text {in }}, r_{\mathrm{c}}, r_{\text {out }}\right)\right\}
$$

\section{Annular Network}

If $\mathcal{A}$ is a disk of radius $r_{\text {out }}$, the outage probability can be found from 2.48 with $r_{\mathrm{c}}=r_{\text {out }}$,

$$
\epsilon_{j}(\mathcal{A})=1-\exp \left\{-\frac{\beta r_{0, j}^{\alpha}}{\mathrm{SNR}}+\pi \lambda p\left[\Phi\left(r_{\text {out }}\right)-\Phi\left(r_{\text {in }}\right)\right]\right\} .
$$

Theorem 2.3.1 When $\mathcal{A}$ includes the interferers on the entire plane $\mathbb{R}^{2}$, the outage probability is

$$
\epsilon_{j}=1-\exp \left\{-\frac{\beta r_{0, j}^{2}}{\mathrm{SNR}}-\frac{2 \pi^{2} \lambda p}{\alpha} \beta^{\frac{2}{\alpha}} r_{0, j}^{2} \csc \left(\frac{2 \pi}{\alpha}\right)\right\}
$$

Proof: The details of the derivation are given in Appendix B using both probability theory, and tools from stochastic geometry [21].

In the absence of noise $(S N R=\infty)$, 2.50) coincides with equation (3.4) of [14 and equation (61) of [52]. The utility of $(2.49)$ is that it generalizes those earlier two equations, as it permits a non-zero exclusion zone, a finite network area, and a finite SNR.

\section{Arbitrary Area}

Assume that the interferers are in an arbitrarily shaped area. Similar to the case when the interferers are distributed according to a $\mathrm{BPP}$, generally the spatially averaged outage probability cannot be evaluated in closed form, but it can be numerically evaluated.

In Rayleigh fading, when the distances are normalized such that the reference transmitter is at unit distance from the receiver and under the assumption that $p_{i}=p$ and $P_{i}=P_{0}$ for all $i \in\{1, \ldots, M\}$, the spatially averaged outage probability can be found by substituting 
2.44 and 2.45 into 2.12 and using the power-series representation of the exponential function:

$$
\epsilon_{j}=1-\exp \left\{-\frac{\beta r_{0, j}^{\alpha}}{\mathrm{SNR}}-p M+p M \int f_{r}(r) \frac{r^{\alpha}}{\beta r_{0, j}^{\alpha}+r^{\alpha}} d r\right\} .
$$

It follows that in this case computing the outage probability for a $\mathrm{PPP}$ reduces to evaluating a single one-dimensional integral, which can be solved either numerically or by a simple Monte Carlo simulation as described in Sec. 2.3.1.

\subsection{Spatial Interference Correlation}

The spatial interference correlation describes how the outages at two different points in space are correlated. Interference correlation arises because a common set of potential interferers are observed through similarly attenuated channels, even if the fading is decorrelated in space and time. The spatial interference correlation can be used to analyze and design multi-hop networks, and compression transmission strategies when cooperation is employed.

The spatial interference correlation is quantified by evaluating the correlation coefficient of the outage probability at two different receivers $\left(Y_{1}\right.$ and $\left.Y_{2}\right)$, which is

$$
\rho\left[\epsilon_{1}(\boldsymbol{\Omega}), \epsilon_{2}(\boldsymbol{\Omega})\right]=\frac{\mathbb{E}_{M, \boldsymbol{\Omega}}\left[\epsilon_{1}(\boldsymbol{\Omega}) \epsilon_{2}(\boldsymbol{\Omega})\right]-\mathbb{E}_{M, \boldsymbol{\Omega}}^{2}\left[\epsilon_{j}(\boldsymbol{\Omega})\right]}{\mathbb{E}_{M, \boldsymbol{\Omega}}\left[\epsilon_{j}^{2}(\boldsymbol{\Omega})\right]-\mathbb{E}_{M, \boldsymbol{\Omega}}^{2}\left[\epsilon_{j}(\boldsymbol{\Omega})\right]}
$$

where $E_{M, \Omega}[\cdot]$ is the expectation operator, evaluated over the number of interferers and their normalized powers, and either $j=1$ or $j=2$ could be used as the two receivers are stochastically equivalent (they experience the same average outage probability).

Similar to the analytical framework described in Sec. 2.2 and Sec. 2.3, the expectations in 2.52 can be first evaluated over the spatial distribution of the interferers, and then over their number. Specifically, the spatially averaged first moment of the outage probability over $\Omega$ conditioned over the number of interferers $M$ can be evaluated by $(2.11)$. The spatially averaged second moment of the outage probability over $\Omega$ conditioned over the number of interferers $M$ is

$$
\mathbb{E}_{\boldsymbol{\Omega}}\left[\epsilon_{j}^{2}(\boldsymbol{\Omega}) \mid M\right]=\int \epsilon_{j}^{2}(\boldsymbol{\Omega}) f_{\boldsymbol{\Omega}}(\boldsymbol{\Omega} \mid M) \mathrm{d} \boldsymbol{\Omega}
$$




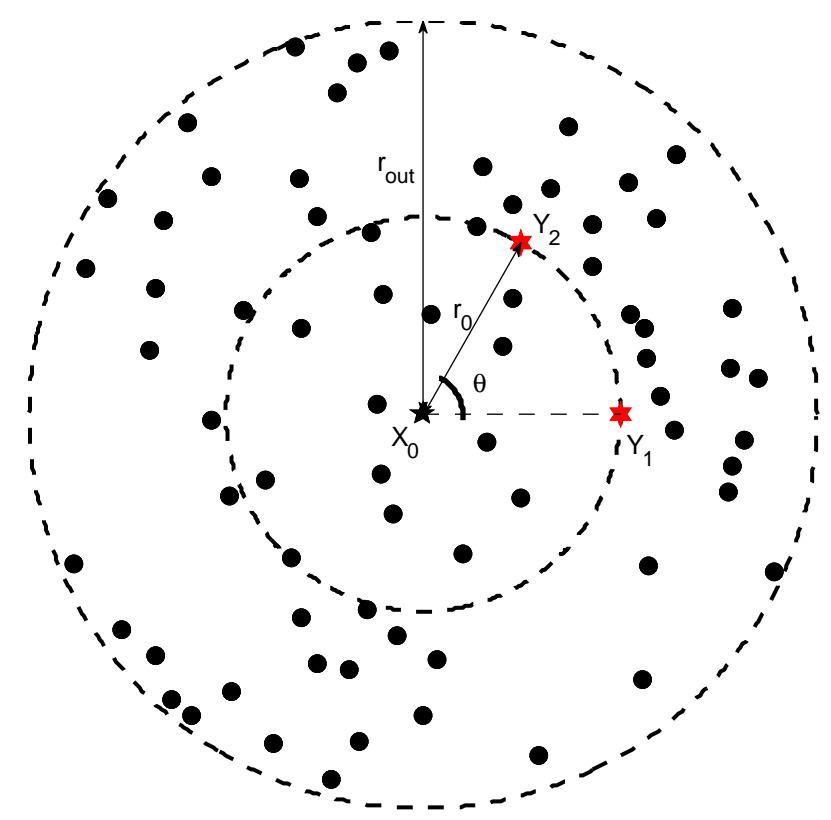

Figure 2.1: Typical network topology. The reference transmitter is located at the center. The two reference receivers are represented by the two red stars on the circumference of radius $r_{0}$.

The spatially averaged first joint moment of the outage probability over $\Omega$ conditioned over the number of interferers $M$ is

$$
\mathbb{E}_{\boldsymbol{\Omega}}\left[\epsilon_{1}(\boldsymbol{\Omega}) \epsilon_{2}(\boldsymbol{\Omega}) \mid M\right]=\int \epsilon_{1}[\boldsymbol{\Omega}] \epsilon_{2}[\boldsymbol{\Omega}] f_{\boldsymbol{\Omega}}(\boldsymbol{\Omega}) \mathrm{d} \boldsymbol{\Omega}
$$

When the number of interferers is random, the overall spatially averaged first, second and first joint moment of the outage probability can be obtained by averaging with respect to $M$. In particular, it is possible to use the following equality

$$
\mathbb{E}_{M, \boldsymbol{\Omega}}\left[\epsilon_{i}^{x}(\boldsymbol{\Omega}) \epsilon_{j}^{y}(\boldsymbol{\Omega})\right]=\sum_{m=0}^{\infty} p_{M}[m] E_{\boldsymbol{\Omega}}\left[\epsilon_{i}^{x}(\boldsymbol{\Omega}) \epsilon_{j}^{y}(\boldsymbol{\Omega}) \mid m\right] .
$$

\subsubsection{Special Cases}

Assume the reference transmitter is located at the center of a circular network of radius $r_{\text {out }}$. Assume the receiver $Y_{1}$ and $Y_{2}$ are located on a circle of radius $r_{0}$ within the network $\left(r_{0,1}=r_{0,2}=r_{0}\right)$ and the vectors connecting their locations to the origin form an angle $\theta$, as depicted in Fig. 2.1. For simplicity of exposition, assume a Rayleigh-faded channel (for 
Nakagami fading, a similar derivation can be performed), an unshadowed scenario. As in the previous sections, assume $P_{i}=P_{0}$ and $p_{i}=p_{0}$ for all $i$.

\section{Binomial Point Process}

Assume that the $M$ interferers are independently and uniformly distributed (i.u.d.) over $\mathcal{A}$. Thus, the interferers are drawn from a $\mathrm{BPP}$ of intensity $\lambda=M /|\mathcal{A}|$.

Theorem 2.4.1 The spatially averaged first moment of the outage probability is

$$
\mathbb{E}_{\boldsymbol{\Omega}}\left[\epsilon_{j}(\boldsymbol{\Omega}) \mid M\right]=1-\exp \left(\frac{\beta r_{0}^{\alpha}}{\mathrm{SNR}}\right)\left\{1-p+\frac{2 p}{|\mathcal{A}|}\left[\frac{\pi}{\beta r_{0}^{\alpha}} \Psi_{1}\left(r_{\text {out }}-r_{0}\right)+\mathcal{Z}_{1}\left(r_{\text {out }}, r_{0}\right)\right]\right\}^{M}
$$

where the function $\mathcal{Z}_{i}\left(r_{\text {out }}, r_{0}\right)$ is given by $\left.C .3\right)$.

Proof: The details of the derivation are given in Appendix $\square$.

Theorem 2.4.2 The spatially averaged second moment of the outage probability is

$$
\mathbb{E}_{\boldsymbol{\Omega}}\left[\epsilon_{j}^{2}(\boldsymbol{\Omega}) \mid M\right]=2 E_{\boldsymbol{\Omega}}\left[\epsilon_{j}(\boldsymbol{\Omega}) \mid M\right]-1+\exp \left(-\frac{2 \beta r_{0}^{\alpha}}{\mathrm{SNR}}\right) \mathcal{S}^{M}\left(r_{\text {out }}, r_{0}\right)
$$

where the function $\mathcal{S}\left(r_{\text {out }}, r_{0}\right)$ is given by $\left.\overline{C .6}\right)$.

Proof: The details of the derivation are given in Appendix $C$.

Theorem 2.4.3 The spatially averaged joint first moment of the outage probability is

$$
\mathbb{E}_{\boldsymbol{\Omega}}\left[\epsilon_{1}(\boldsymbol{\Omega}) \epsilon_{2}(\boldsymbol{\Omega}) \mid M\right]=2 E_{\boldsymbol{\Omega}}\left[\epsilon_{j}(\boldsymbol{\Omega}) \mid M\right]-1+\exp \left(-\frac{2 \beta r_{0}^{\alpha}}{\mathrm{SNR}}\right) \mathcal{W}^{M}\left(r_{\text {out }}, r_{0}, \theta\right)
$$

where the function $\mathcal{W}\left(r_{\text {out }}, r_{0}, \theta\right)$ is given by C.11).

Proof: The details of the derivation are given in Appendix $\square$.

Finally, by substituting 2.56), 2.57) and (2.58) in (2.52), the correlation coefficient can be evaluated.

\section{Poisson Point Process}

Suppose that the interferers are drawn from a $\mathrm{PPP}$ with intensity $\lambda$ within an circular region of radius $r_{\text {out }}$. Set $x=0$ and $y=1$ in (2.55), and substitute (2.45), and (2.56) within 
it. By applying the power-series representation of the exponential function, the spatially averaged first moment of the outage probability is

$$
\mathbb{E}_{M, \Omega}\left[\epsilon_{i}(\Omega)\right]=1-\exp \left\{-\frac{\beta r_{0}^{\alpha}}{\operatorname{SNR}}-2 \lambda p\left[\frac{|\mathcal{A}|}{2}-\frac{\pi}{\beta r_{0}^{\alpha}} \Psi_{1}\left(r_{\text {out }}-r_{0}\right)+\mathcal{Z}_{1}\left(r_{\text {out }}, r_{0}\right)\right]\right\} .
$$

Set $x=0$ and $y=2$ in (2.55), and substitute (2.45) and 2.57) within it. By applying again the power-series representation of the exponential function, the spatially averaged second moment of the outage probability is

$$
\mathbb{E}_{M, \Omega}\left[\epsilon_{i}^{2}(\boldsymbol{\Omega})\right]=2 \mathbb{E}_{M, \Omega}\left[\epsilon_{i}(\boldsymbol{\Omega})\right]-1+\exp \left\{-\frac{2 \beta r_{0}^{\alpha}}{\mathrm{SNR}}-\lambda|\mathcal{A}|\left[1+\mathcal{S}\left(r_{\text {out }}, r_{0}\right)\right]\right\}
$$

Set $x=1$ and $y=1$ in (2.55), and substitute 2.45) and 2.58 within it. By using the power-series representation of the exponential function, the spatially averaged first joint moment of the outage probability is

$$
\mathbb{E}_{M, \boldsymbol{\Omega}}\left[\epsilon_{1}(\boldsymbol{\Omega}) \epsilon_{2}(\boldsymbol{\Omega})\right]=2 E_{M, \boldsymbol{\Omega}}\left[\epsilon_{i}(\boldsymbol{\Omega})\right]-1+\exp \left\{-\frac{2 \beta r_{0}^{\alpha}}{\mathrm{SNR}}-\lambda|\mathcal{A}|\left[1+\mathcal{W}\left(r_{\text {out }}, r_{0}, \theta\right)\right]\right\}
$$

Finally, by substituting (2.59), 2.60 and 2.61) in 2.52), the correlation coefficient can be evaluated.

\subsection{Numerical Results}

In this section, numerical results are provided and, unless otherwise stated, it is assumed that the network consists of a source transmitter placed at unit distance from the receiver (i.e., $r_{0, j}=1$ ) and $\mathrm{M}$ interferers placed independently according to a given spatial distribution in an annular region with inner dimension $r_{\text {in }}=0.25$ and outer dimension $r_{\text {out }}=4$. Furthermore $\Omega$ is evaluated by assuming a path loss exponent $\alpha=3$, a common transmit power $P_{i}=P_{0}$, and the absence of shadowing. The activity probability is common for all the transmitters $p_{i}=p$, and the SINR threshold is set to $\beta=3.7 \mathrm{~dB}$.

Fig. 2.2 shows the conditional outage probability $\epsilon(\Omega)$ when the common activity probability is $p=1 / 200$ and fifty interferers are independently placed according to a $\mathrm{BPP}$ in an annular region as shown in the inset as function of SNR for three cases: 1) all channels are Rayleigh faded; 2) Nakagami fading with all channels $m_{i, j}=4$, which represents the 


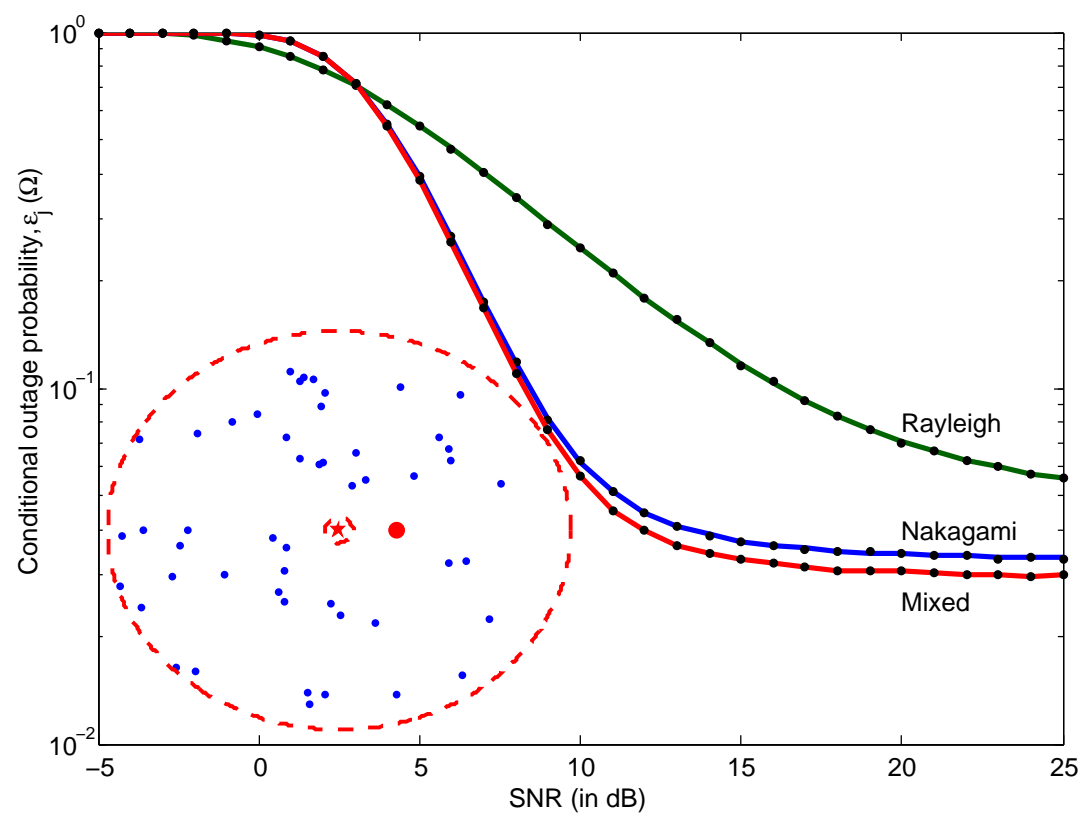

Figure 2.2: Conditional outage probability $\epsilon_{j}(\boldsymbol{\Omega})$ as a function of SNR. Analytical curves are solid while dots represent simulated values. Top curve: all channels Rayleigh. Middle curve: all channels $m_{i, j}=4$. Bottom curve: $m_{0, j}=4$ for the source, and $m_{i, j}=1$ for the interferers. The network topology is shown in the inset. The receiver is represented by the star at the center of the radius- 4 circle, the desired transmitter is the big red dot, while the 50 interferers are shown as blue dots.

situation where the source and interferer are equally visible to the receiver; 3) Mixed fading with $m_{0, j}=4$ for the source and $m_{i, j}=1$ for the interferers, which represents a more typical situation where the interferers are not in the LOS, The three curves are obtained by using 2.9). Also shown are curves generated by simulation, which involve randomly generating the Nakagami-distributed $\left\{g_{i, j}\right\}$ and selecting the interferers from a uniform distribution. The analytical and simulation results coincide, which is to be expected because $(2.9)$ is exact. Any discrepancy between the curves can be attributed to the finite number of Monte Carlo trials (one million trials were executed per SNR point).

The spatially averaged outage probability for a $\mathrm{BPP}$ is shown as a function of $M$ for two values of $p$ in Fig. 2.3. A mixed-fading model is assumed, i.e. $m_{0, j}=4$ and $m_{i, j}=1$ for $i \geq 1$, and SNR $=10 \mathrm{~dB}$ is fixed. For each value of $p$, three curves are shown, one without shadowing and two with shadowing. To model log-normal shadowing, each $\xi_{i, j}$ is drawn from a zero-mean Gaussian distribution with standard deviation $\sigma_{s}$ that varies among the set $\sigma_{s}=\{4,8\}$ dB. From Fig. 2.3, it is observed that the outage probability degrades 


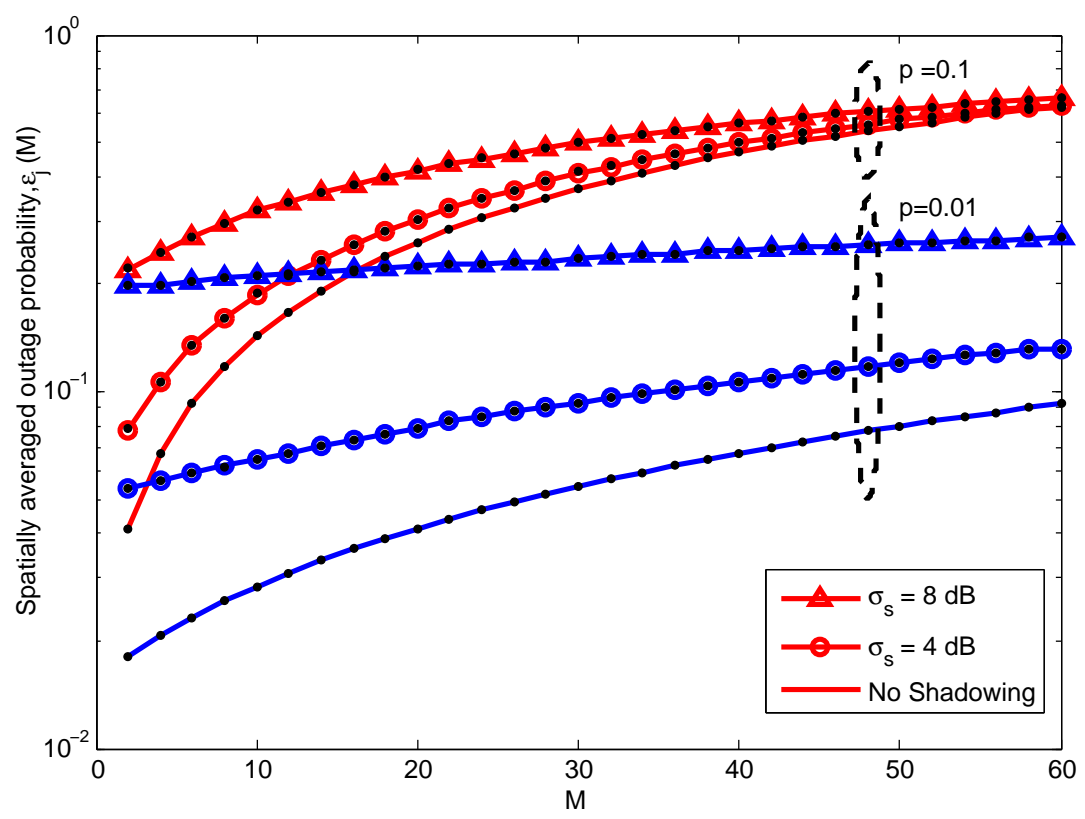

Figure 2.3: Spatially averaged outage probability for a BPP as a function of the number of interferers $M$ for two values of $p$. For each $p$, curves are shown for the case of no shadowing, and for shadowing with two values of $\sigma_{s}$.

with increasing $M$ and decreasing $p$, and that shadowing increases the average $\epsilon(M)$ with increasing $\sigma_{s}$ for the given parameters.

Fig. 2.4 shows the spatially averaged outage probabilities in unshadowed Rayleigh fading as a function of $\lambda p$, when the interfering devices are placed according to a $\mathrm{PPP}$ with intensity $\lambda$ in a circular region of radius $r_{\text {out }}\left(r_{\text {in }}=0\right)$ and SNR $=10 \mathrm{~dB}$. Four network radii are considered: $r_{\text {out }}=\{1,2,4, \infty\}$. The outage probabilities of the three networks with finite radius were computed using (2.49), while the outage probability of the infinite network (shown by the dashed curve) was computed using (2.50). In addition, simulation results (shown in this figure by dots) are shown for the three finite networks, which coincide with the theoretical curves. For the simulations, one million trials were executed per $\lambda$ point. Any discrepancy between the theoretical result and the simulation is due to the finite number of trials. Fig. 2.4 exhibits the effect of the finite spatial extent of a network and shows that the outage probability increases with increasing $\lambda p$ and/or increasing $r_{\text {out }}$.

The performances are compared when the reference receiver is at the center of the three different shapes, which are shown in the inset of Fig. 2.5. The shapes are a disk of radius $r_{\text {out }}=2$, a triangle ( $L=3$ polygon), and an arbitrary shape, which happens to look like a 


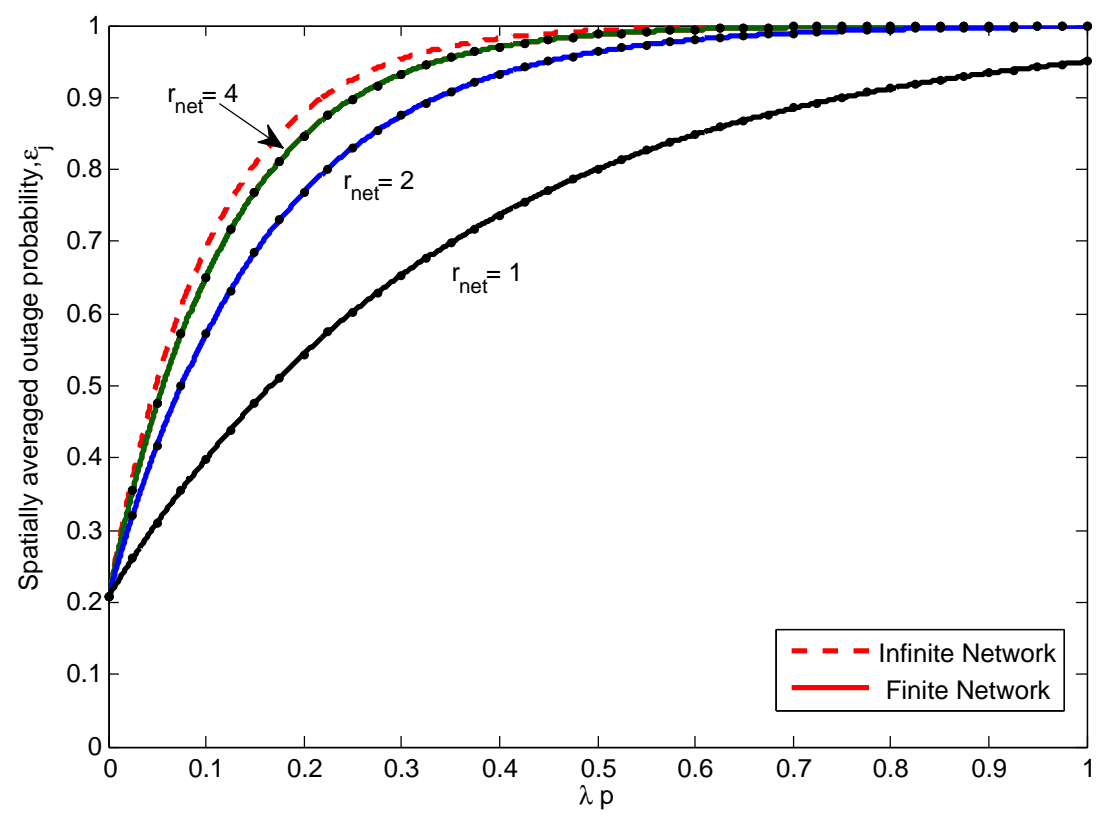

Figure 2.4: Spatially averaged outage probability with interfering devices placed according to a PPP plotted as a function of the effective spatial density of interfering devices $\lambda p$. Curves are shown for three finite network radii $\left(r_{\text {out }}=[1,2,4]\right)$ and for the asymptotic case $\left(r_{\text {out }} \rightarrow \infty\right)$. The fading is Rayleigh, and there is no shadowing. Dots indicate the results of Monte Carlo simulation while the curves are computed from the theoretical expressions.

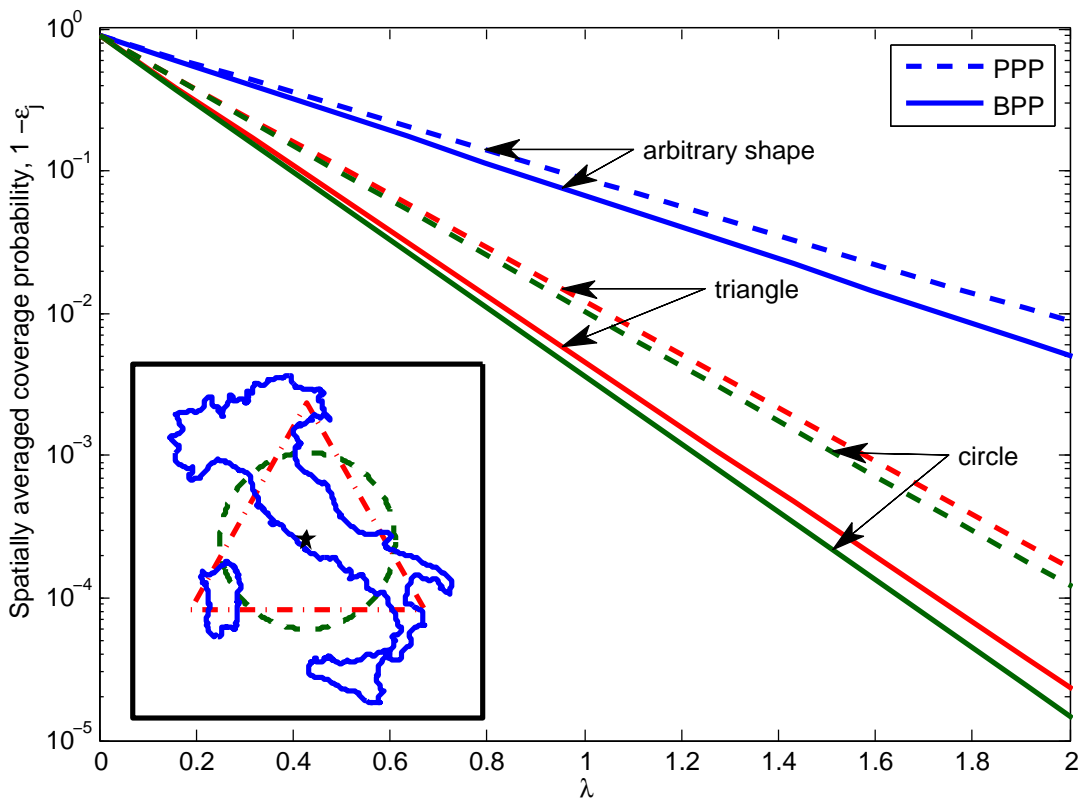

Figure 2.5: Coverage probability $\overline{\epsilon_{j}}=1-\epsilon_{j}$ as function of the network density $\lambda$. Results are shown for the case that the interferers are drawn from a BPP (solid lines) and from a PPP (dashed lines). From highest to lowest coverage probability, the curves correspond to the arbitrary shape (blue lines), the triangle (red lines), and the circle (green lines). 


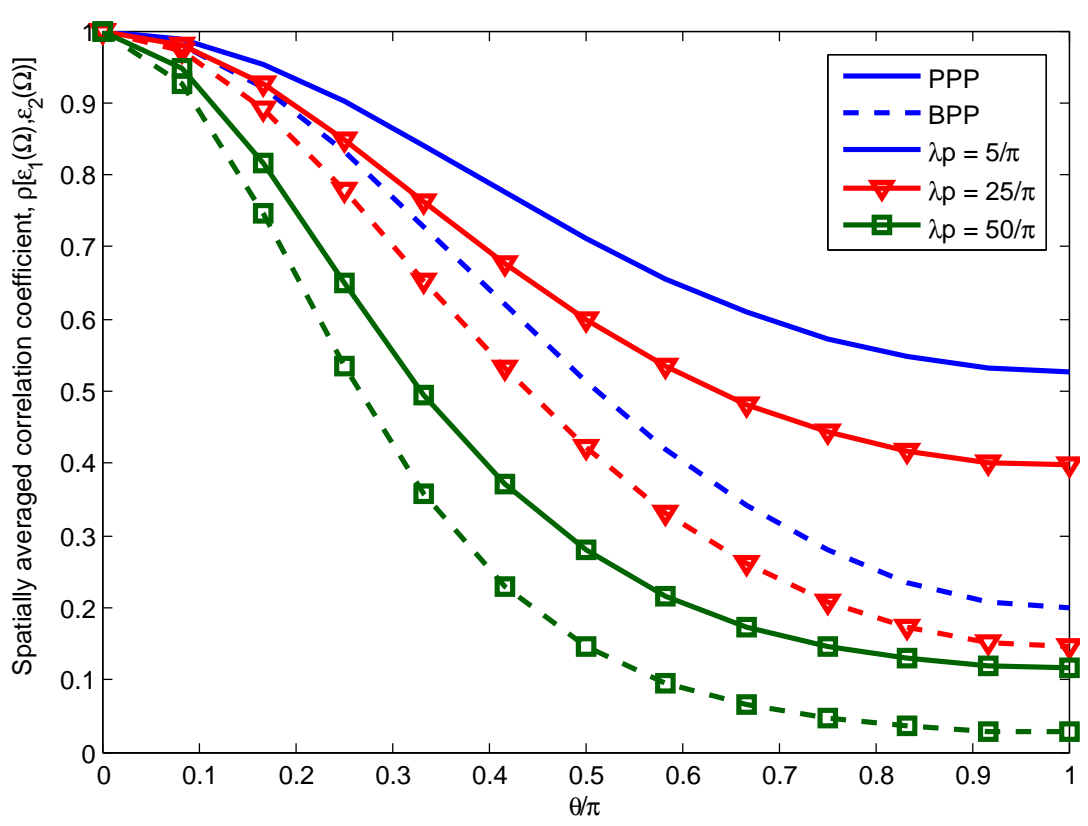

Figure 2.6: Spatially averaged correlation coefficient as function of $\theta / \pi$. Results are shown for the case that the interferers are drawn from a BPP (solid lines) and from a PPP (dashed lines) and for three values of $\lambda p$.

map of Italy including the islands of Sicily and Sardinia (emphasizing that $\mathcal{A}$ need not be contiguous). In all cases, the shapes are scaled so that they have the same area, $|\mathcal{A}|=4 \pi$, and there is no exclusion zone around the receiver; i.e., $r_{\text {in }}=0$. The spatially averaged outage probability for each network was computed for interferer densities $\lambda \leq 2$, path loss exponent $\alpha=3.2$, threshold $\beta=0 \mathrm{~dB}$, and average SNR $=10 \mathrm{~dB}$. Results are shown for both the $\mathrm{BPP}$ and the $\mathrm{PPP}$ cases. For the triangle, the series in 2.27 is truncated at the $25^{\text {th }}$ term. For the arbitrary region, the integral in $(2.43)$ and $(2.51$ is evaluated using a fine grid of 125, 640 equally spaced points. The spatially averaged coverage probabilities, defined as $\bar{\epsilon}_{j}=1-\epsilon_{j}$ are shown in Fig. 2.5. As can be seen, the disk-shaped network has the worst spatially averaged coverage probability, while those that depart from a circle have better performance. The spatially averaged coverage probability of the $\mathrm{PPP}$ is better than that of the BPP. The irregularly shaped network has a very high spatially averaged coverage probability, suggesting that the common assumption of a disk shaped network is overly pessimistic for realistic networks with highly irregular shapes.

Finally, the spatially averaged correlation coefficient of the outage probability as a function of the angle $\theta$ formed between two receivers and the reference transmitter is shown in 
Fig. 2.6. Results are shown in unshadowed Rayleigh fading for both the BPP and PPP cases and for three values of $\lambda p$. The network is circular with radius $r_{\text {out }}=1$, and both receivers are at distance $r_{0}=0.25$ from the reference transmitter located at the center. Fig. 2.6 shows that the outage probability is more correlated in a sparse network. This is because in a sparse network, it is more likely that the same interferer will dominate at both receiver locations. This is in contrast with a dense network, where the dominant interferer at the first receiver location is usually different than the dominant interferer at the other location. This is because in a sparse network, it is more likely that the same interferer will dominate at both receiver locations. This is in contrast with a dense network, where the dominant interferer at the first receiver location is usually different than the dominant interferer at the other location. Furthermore, Fig. 2.6 shows that a $\mathrm{PPP}$ experiences a higher correlation than a $\mathrm{BPP}$, which might seam counterintuitive since a $\mathrm{PPP}$ adds an additional degree of diversity due to the stochastic nature of the number of interference involved in the transmission. However, even if the values of the outage probability might vary more under a PPP between one snapshot to another, this type of process can lead to snapshots of the network in which the interferers are sparser than those obtained with a $\mathrm{BPP}$, and this scenario as explained above is characterized by a higher correlation.

\subsection{Chapter Summary}

While the standard way to find the spatially averaged outage probability is to use the LT and PGFL, as outlined in [27], it is possible to obtain the same result in a more direct manner. This chapter introduced a new analytical framework for computing the spatially averaged outage probability of a network with interferers located according to a point process and signals subject to fading. The starting point is to find the outage probability conditioned on the network topology and shadowing factors. Next, the number of interferers is fixed and the outage probability is averaged with respect their random positions and eventually shadowing. The result is the outage probability for a BPP. Then, the outage probability for $\mathrm{a} \mathrm{BPP}$ is marginalized with respect to the number of interferers to get the outage probability for a $\mathrm{PPP}$, which generalizes the case when the network extends over the entire plane. In 
fact, by allowing the network to be infinite, the classic result of Baccelli et al. 14, 52 is obtained.

The main benefit of this approach is that it breaks the analysis into discrete steps that consider the fading and the random topology separately, in contrast with lumping them together as done by using tools from stochastic geometry [21]. This is useful, since the two effects have a different timescales, and, in particular, fading changes more rapidly than the locations of the interferers. Another benefit is that this approach provides a way to deal with spatial distributions that are not mathematically tractable by using a Monte Carlo simulation, without the need to average over the fading. Furthermore, the spatially averaged outage probability in Rayleigh fading reduces to a single one-dimensional integral for both a $\mathrm{BPP}$ and a $\mathrm{PPP}$, which when closed form expression cannot be found, it can be simply evaluated either numerically or by a Monte Carlo simulation of the location of a single interferer.

Finally, this chapter concludes by applying the aforementioned analytical framework in order to quantify the effect of the spatial interference correlation and semi-closed form expressions are derived. Despite the fact that a direct approach is used, a key difference with the previous work is that the interference correlation is quantified analytically for finite networks, instead of relying on the common assumption of an infinite network. 


\section{Chapter 3}

\section{Cellular Networks: Modeling and Analysis}

This chapter applies the analytical framework developed in Chap. 2 to accurately model and analyze cellular networks, by capturing the effect of the interference and the interdependence among some of the system parameters. This chapter provides a performance analysis of the uplink of a DS-CDMA cellular network under different resource allocation policies. While the focus of this chapter is on the uplink, a similar model and analysis can be also applied to the downlink [53]. Furthermore, as will be more evident in this chapter, the methodology described here is very general, and can be adapted to study the performance of a large variety of MAC protocols and resource allocation policies.

The methodology introduced here [54,55] is able to discharge some of the unrealistic assumptions that are often made in literature, such as: 1) infinite network both in extent

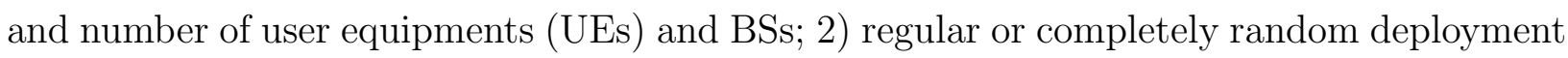
of the network, which poorly mimics a real deployment; 3) circular or regular shaped radio cells where the spatial distribution of both $\overline{\mathrm{BSS}}$ and $\mathrm{UE} ;$ are independent; 4) identical fading conditions for each link; 5) path loss model characterized by a fixed path-loss exponent; 6) absence of shadowing; 7) neglect of thermal noise. 


\subsection{Modeling and Analysis for Cellular Networks}

A cellular network is a wireless network that is distributed over land areas called cells, each served by at least one fixed-location transceiver, known as a BS. A sector is defined as the range of angles from which a directional sector antenna can receive signals. A UE within the sector of a sector antenna is said to be covered by the sector antenna. Cells may be divided into sectors by using several directional sector antennas or arrays, each covering disjoint angles, at the BSs.

The classical analysis of the cellular downlinks (e.g., [56]) and uplinks (e.g., [57 59]) in a cellular network entails a number of questionable assumptions, including the existence of a lattice or regular grid of $\overline{\mathrm{BS}}$ s and the modeling of intercell interference at a $\mathrm{BS}$ as a fixed fraction of the total interference. Although conceptually simple and locally tractable, the grid assumption is a poor model for actual BS deployments, which cannot assume a regular grid structure due to a variety of regulatory and physical constraints. The intercell-interference assumption is untenable because the fractional proportion of intercell interference varies substantially with the $\mathrm{UE}$ and $\mathrm{BS}$ locations, the shadowing, and the fading.

More recent analyses of cellular networks (e.g., [60 66]) locate the पUEs and/or the [BS; according to a two-dimensional $\mathrm{PPP}$ over a network that extends infinitely on the Euclidian plane, thereby allowing the use of analytical tools from stochastic geometry. Although the two principal limitations of the classical approach are eliminated, the PPP approach is still unrealistic because it does not permit a minimum separation between $\mathrm{BS}$; or between UEs. However, both the minimum separations are characteristic of actual macro-cellular deployments. Other problems with the PPP model are that no network has an infinite area and that any realization of a $\mathrm{PPP}$ could have an implausibly large number of $\overline{\mathrm{UE}} ; \mathrm{s}$ or $\mathrm{BS} ;$ placed within a finite area.

When tools from stochastic geometry are used in order to considerably simplify the tractability of the problem, the adoption of a simple point process to model the spatial distribution of both $\mathrm{BS}$ and $\mathrm{UE} s$ is not the only unrealistic assumption. In this case, in fact, it is often assumed [65] that the radio cells are circular, and the spatial distribution of the UEs and $\overline{\mathrm{BS}} \mathrm{s}$ are independent. This last assumption may lead to snapshots of the network where 
there are several overlapping cells, which is not realistic for macro-cellular deployments, while however might well model an heterogeneous cellular network. For the aim of tractability, often the effect of thermal noise and shadowing are also neglected. Furthermore, it is often assumed identical fading conditions for each link and the analysis is constrained to the use of a standard distance-dependent path loss model with a single slope or more recently with the dual-slope [67]. While these two assumptions are widely used to design, model and analyze cellular networks, they do not capture adequately the dependency of the fading and path loss on the link distance, which is extremely important to properly model and analyze densely deployed and millimeter wave cellular networks [68, 69].

This chapter introduces a methodology based on the analysis built in Chap. 2, which relaxes all the aforementioned assumptions to more realistically model cellular networks.

\subsection{Network Model}

Practical sector antennas have patterns with sidelobes that extend into adjacent sectors, but the performance degradation due to overlapping sectors is significant only for a small percentage of UE locations. Three ideal sector antennas and sectors per BS, each covering $2 \pi / 3$ radians, are assumed in this section. The UE antennas are assumed to be omnidirectional. The UEs in the directions covered by a sector antenna can cause intracell or intercell multiple-access interference from a UE to its associated sector antenna, as illustrated in Fig. 3.1. Thus, the number of interfering signals on the uplink is reduced approximately by a factor equal to the number of sectors.

In this section, assume the network comprises $K \overline{\mathrm{BS}}$ s and cells, $S_{\text {sector }}=3 K$ sectors $\left\{Y_{1}, \ldots, Y_{S_{\text {sector }}}\right\}$, and $M$ UE: $\left\{X_{1}, \ldots, X_{M}\right\}$. The $\mathrm{BS}$ and $\mathrm{UE}$ are confined to a finite arena of area $|\mathcal{A}|$. The sector boundary angles are the same for all $\overline{\mathrm{BSS}} \mathrm{s}$. Let $\mathcal{Y}_{j}$ denote the set of UE covered by sector antenna $Y_{j}$ and $\mathcal{M}_{j}=\left|\mathcal{Y}_{j}\right|$ be the number of UE served by $Y_{j}$. A पE $X_{i} \in \mathcal{Y}_{j}$ will be associated with $Y_{j}$ if the $\overline{\mathrm{UE}}$ s signal is received at $Y_{j}$ with a higher average power than at any other sector antenna in the network. Let $\mathcal{X}_{j} \subset \mathcal{Y}_{j}$ denote the set of $\mathrm{UE} ;$ associated with sector antenna $Y_{j}$. Furthermore, let $\mathrm{g}(i)$ be a function that returns the index of the sector serving $X_{i}$ so that $X_{i} \in \mathcal{Y}_{j}$ if $\mathrm{g}(i)=j$. If $X_{i}$ cannot connect to any 


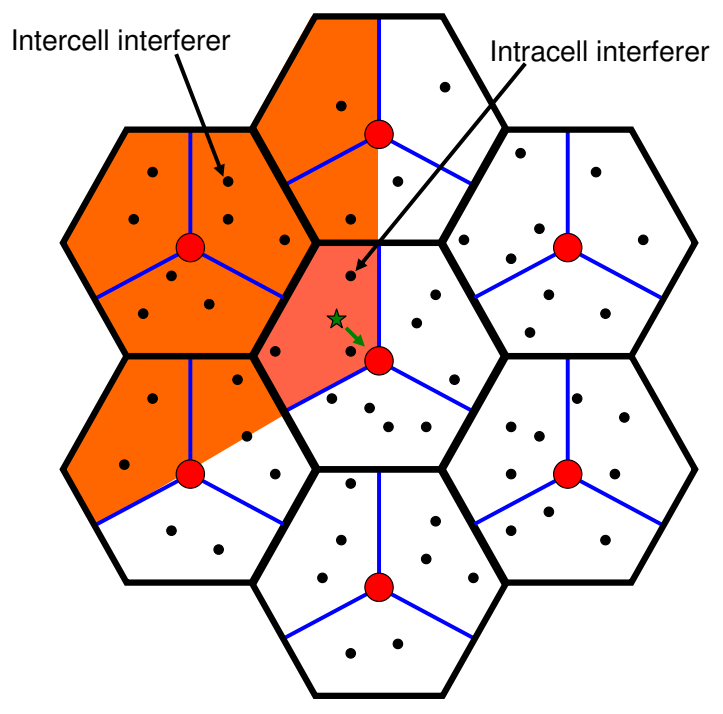

Figure 3.1: Illustration of intracell (all the UEs within the light red area) and intercell (all UEs within the orange area) interference. BSs are represented by large circles, and UEs are represented by black dots with the exception of the reference UE which is a green star. Cell boundaries are represented by thick blue lines.

BS, which is possible when a cell runs out of available channels, then $\mathrm{g}(i)=0$.

The power of $Y_{j}$ received at $X_{i}$ depends on the fading and path loss models and as in the previous chapter it is assumed that path loss has a power-law dependence on distance and is perturbed by shadowing. The sector antenna $Y_{\mathrm{g}(i)}$ that serves $\mathrm{UE} X_{i}$ is selected to be the one with index

$$
\mathrm{g}(i)=\underset{j}{\operatorname{argmax}}\left\{10^{\xi_{i, j} / 10} r_{i, j}^{-\alpha}\right\}
$$

which is the sector antenna with minimum path loss to $X_{i}$. In the presence of shadowing, a UE may actually be associated with a sector antenna that is more distant than the closest one, if the shadowing conditions are sufficiently better.

Assume the use of orthogonal DS-CDMA, although the approach used here is general enough to be able to model other types of access, such as orthogonal frequency-division multiple access (QFDMA). Let $X_{o} \in \mathcal{X}_{j}$ denote a reference UE that transmits a desired signal to $Y_{j}$. Both intracell and intercell interference are received from other UEs within the covered angle of the sector, but interference from UE in extraneous sectors is negligible, since duplexing is assumed to prevent it. The varying propagation delays from the interfering 
UE cause their interference signals to be asynchronous with respect to the desired signal. The power of $X_{i}, i \neq o$, received at $Y_{j}$ is nonzero only if $X_{i} \in \mathcal{Y}_{j}$ and in this case it is effected by the processing gain $G$. The power before despreading from multiple-access interferers is in fact reduced after despreading by the effective processing gain $G_{e}=G / c_{f}\left(\tau_{o}\right)$, where $c_{f}\left(\tau_{o}\right)$ is function of the chip waveform and the timing offset $\tau_{o}$ of the interference spreading sequence relative to that of the desired or reference signal. If $\tau_{o}$ is assumed to have a uniform distribution over $\left[0, T_{c}\right]$ then the expected value of $c_{f}\left(\tau_{o}\right)$ is the chip factor $c_{f}$. Henceforth, it is assumed that $G / c_{f}\left(\tau_{o}\right)$ is a constant equal to $G / c_{f}$ at each receiver in the network, and the normalized received power becomes

$$
\Omega_{i, j}= \begin{cases}10^{\xi_{i, j} / 10} r_{i, j}^{-\alpha} & i=o \\ \frac{h P_{i}}{G P_{0}} 10^{\xi_{i, j} / 10} r_{i, j}^{-\alpha} & i: X_{i} \in \mathcal{Y}_{j} \backslash X_{o} \\ 0 & i: X_{i} \notin \mathcal{Y}_{j}\end{cases}
$$

Assuming the channel gains remain fixed for the duration of a time interval, but vary independently from interval to interval (block fading), by substituting (3.2) in (2.9), it is possible to evaluate the conditional outage probability for each uplink, given a certain topology of the network.

\subsection{Spatial Model}

In order to model more realistically the spatial distribution of both $\mathrm{BS} ;$ and UE; in a cellular network a uniform clustering model [46] is adopted. The uniform clustering model is a constrained spatial model, that requires a minimum separation between interferers. An exclusion zone of radius $r_{\mathrm{ex}_{\mathrm{bs}}}$ surrounds each $\overline{\mathrm{BS}}$, and no other $\mathrm{BS}$ s are allowed within this zone. Similarly, an exclusion zone of radius $r_{\mathrm{ex}_{\mathrm{mb}}}$ surrounds each UE where no other UEs are allowed. The minimum separation between $\overline{\mathrm{UE}} \mathrm{s}$ is generally much smaller than the minimum separation between the $\mathrm{BS}$ s. BS exclusion zones are primarily determined by economic considerations and the terrain, whereas UE exclusion zones are determined by the need to avoid physical collision. The value of $r_{\text {ex }}$ could be empirically determined by examining the locations of $\overline{\mathrm{BS}} ;$ in a real network. The value of $r_{\text {ex }}$ could be selected to 

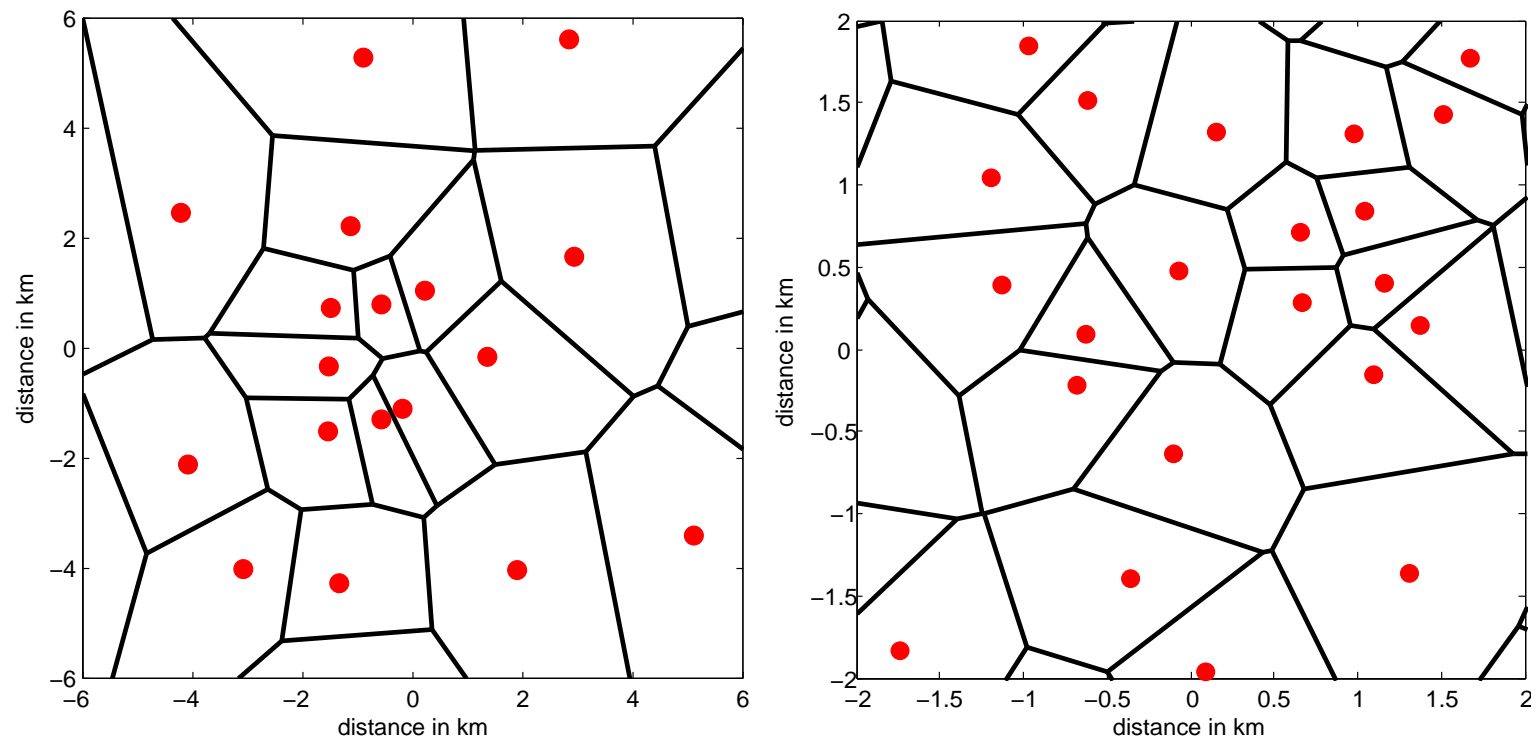

Figure 3.2: Example network topologies. BSs are represented by large circles, and cell boundaries are represented by thick lines. Left subfigure: Actual BS locations from a current cellular deployment. Right subfigure: Simulated BS locations using a BS exclusion zone $r_{\text {ex }}=0.25$.

provide the best statistical fit. This procedure is similar to determining values for fading and shadowing parameters by statistically fitting actual data.

Example network topologies are shown in Fig. 3.2. The left subfigure shows the locations of actual BS; in a small city with hilly terrain. The BS locations are given by the large filled circles, and the Voronoi cells are indicated in the figure. The minimum BS separation is observed to be about $0.43 \mathrm{Km}$. The right subfigure shows a portion of a randomly generated network with a $\mathrm{BS}$ exclusion radius $r_{\text {ex }}=0.25 \mathrm{Km}$. The topological similarity of the two subfigures supports the use of the uniform-clustering model.

\subsection{Resource Allocation Policies}

\subsubsection{Power Control}

A typical power-allocation policy for DS-CDMA networks is to select the transmit power $\left\{P_{i}\right\}$ for all UE in $\mathcal{X}_{j}$ such that, after compensation for shadowing and power-law attenuation, each UE s transmission is received at sector antenna $Y_{j}$ with the same power $P_{0}$, which 
is called full power control. In general, a $\mathrm{UE}$ in $\mathcal{X}_{j}$ can transmits with power $P_{i}$ that satisfies

$$
P_{i} 10^{\xi_{i, j} / 10} r_{i, j}^{-\alpha s}=P_{0}, \quad X_{i} \in \mathcal{X}_{j}
$$

where $s, 0 \geq s \leq 1$, is the compensation factor for fractional power control, which is set to $s=1$ when full power control is adopted.

To accomplish the full power-control policy, sector-antenna receivers estimate the average received powers of their associated UE: Feedback of these estimates enables the associated UE: to change their transmitted powers so that all received powers are approximately equal [70].

For a reference $\mathrm{UE} X_{o}$, the interference at sector antenna $Y_{j}$ is from the UE; in the set $\mathcal{Y}_{j} \backslash X_{o}$. This set can be partitioned into two subsets. The first subset $\mathcal{X}_{j} \backslash X_{o}$ comprises the intracell interferers, which are the other UE; in the same cell and sector as the reference UE. The second subset $\mathcal{Y}_{j} \backslash \mathcal{X}_{j}$ comprises the intercell interferers, which are the UE by sector antenna $S_{j}$ but associated with a cell sector other than $\mathcal{X}_{j}$.

Considering intracell interference, all of the UE within the sector transmit with power given by $(3.3)$. Since $P_{0}$ and $P_{i}$ are obtained from $(3.3)$, the middle line of $(3.2)$ gives the normalized mean received power of the intracell interferers:

$$
\Omega_{i, j}=\frac{h}{G} 10^{\xi_{o, j} / 10} r_{o, j}^{-\alpha}, \quad X_{i} \in \mathcal{X}_{j} \backslash X_{o} .
$$

Although the number of $\mathrm{UE} ; \mathcal{M}_{j}$ in the cell sector must be known to compute the outage probability, the locations of these $\mathrm{UE}$ in the cell are irrelevant to the computation of the $\Omega_{i, j}$ of the intracell interferers.

Considering intercell interference, the set $\mathcal{Y}_{j} \backslash \mathcal{X}_{j}$ can be further partitioned into sets $\mathcal{Y}_{j} \cap \mathcal{X}_{k}, k \neq j$, containing the $\overline{\mathrm{UE}}$ s covered by sector antenna $Y_{j}$ but associated with some other sector antenna $Y_{k}$. For those $\mathrm{UE}$ in $\mathcal{Y}_{j} \cap \mathcal{X}_{k}$, power control implies that

$$
P_{i} 10^{\xi_{i, k} / 10} r_{i, k}^{-\alpha}=P_{0}, \quad X_{i} \in \mathcal{X}_{k} \cap \mathcal{Y}_{j}, \quad k \neq j .
$$

Substituting (3.5), and (3.3) with $i=o$, into 3.2 yields

$$
\begin{aligned}
\Omega_{i, j} & =\frac{h}{G} 10^{\xi_{i, j}^{\prime} / 10}\left(\frac{r_{i, j} r_{o, j}}{r_{i, k}}\right)^{-\alpha} \\
\xi_{i, j}^{\prime} & =\xi_{i, j}+\xi_{o, j}-\xi_{i, k}, \quad X_{i} \in \mathcal{X}_{k} \cap Y_{j}, \quad k \neq j
\end{aligned}
$$


for $\mathcal{Y}_{j} \backslash \mathcal{X}_{j}$, which gives the normalized mean intercell interference power at the reference sector antenna due to interference from $\mathrm{UE} i$ of sector $k=\mathrm{g}(i)$.

\subsubsection{Rate Control}

In addition to controlling the transmitted power, the rate $R_{i}$ of each uplink needs to be selected. Due to the irregular network geometry, which results in cell sectors of variable areas and numbers of $\overline{\mathrm{UE}}$, the amount of received interference can vary dramatically from one sector antenna to another. With a fixed rate, or equivalently, a fixed SINR threshold $\beta$ for each sector, the result is a highly variable outage probability. An alternative to using a fixed rate for the entire network is to adapt the rate of each uplink to satisfy an outage constraint or maximize the throughput of each uplink 71, 72].

To illustrate the influence of rate on performance, consider the following example, where the following scenario is used also in the remaining of this section, if not otherwise stated. The network has $K=50 \widehat{\mathrm{BS}}$ s and $M=400 \mathrm{UE}$ s placed in a circular network of radius $r_{\text {out }}=2$. The $\mathrm{BS}$ exclusion zones have radius $r_{\mathrm{ex}_{\mathrm{bs}}}=0.25$, and the UE exclusion zones have radius $r_{\mathrm{ex}_{\mathrm{mb}}}=0.01$. The spreading factor is $G=16$, and under the assumption of rectangular chip waveforms, $h=2 / 3[73,74$. Since $M / K=G / 2$, the network is characterized as being half loaded. The $\mathrm{SNR}$ is $\mathrm{SNR}=10 \mathrm{~dB}$, the activity factor is $p_{i}=1$, the path loss exponent is $\alpha=3^{1}$, and lognormal shadowing is assumed with standard deviation $\sigma_{s}=8 \mathrm{~dB}$. A distance-dependent fading model is assumed, where a signal originating the $\mathrm{UE} X_{i}$ arrives at the $\mathrm{BS} Y_{j}$ with a Nakagami fading parameter $m_{i, j}$ that depends on the distance between the UEs. In particular,

$$
m_{i, j}= \begin{cases}3 & \text { if } r_{i, j} \leq r_{\mathrm{los}} / 2 \\ 2 & \text { if } r_{\mathrm{los}} / 2<r_{i, j} \leq r_{\mathrm{los}} \\ 1 & \text { if } r_{i, j}>r_{\mathrm{los}}\end{cases}
$$

where $r_{\text {los }}$ is the LOS radius. The distance-dependent-fading model characterizes the situations where $\mathrm{UE} ;$ close to the $\mathrm{BS}$ are in $\mathrm{LOS}$, but $\mathrm{UE} ;$ farther away are not.

Fig. 3.3 shows the outage probability as a function of rate. Assuming the use of a

\footnotetext{
${ }^{1}$ According to the analysis, note that the value of $\alpha$ doesn't not need to be fixed for all the links and can be provided based on a distance-dependent model.
} 


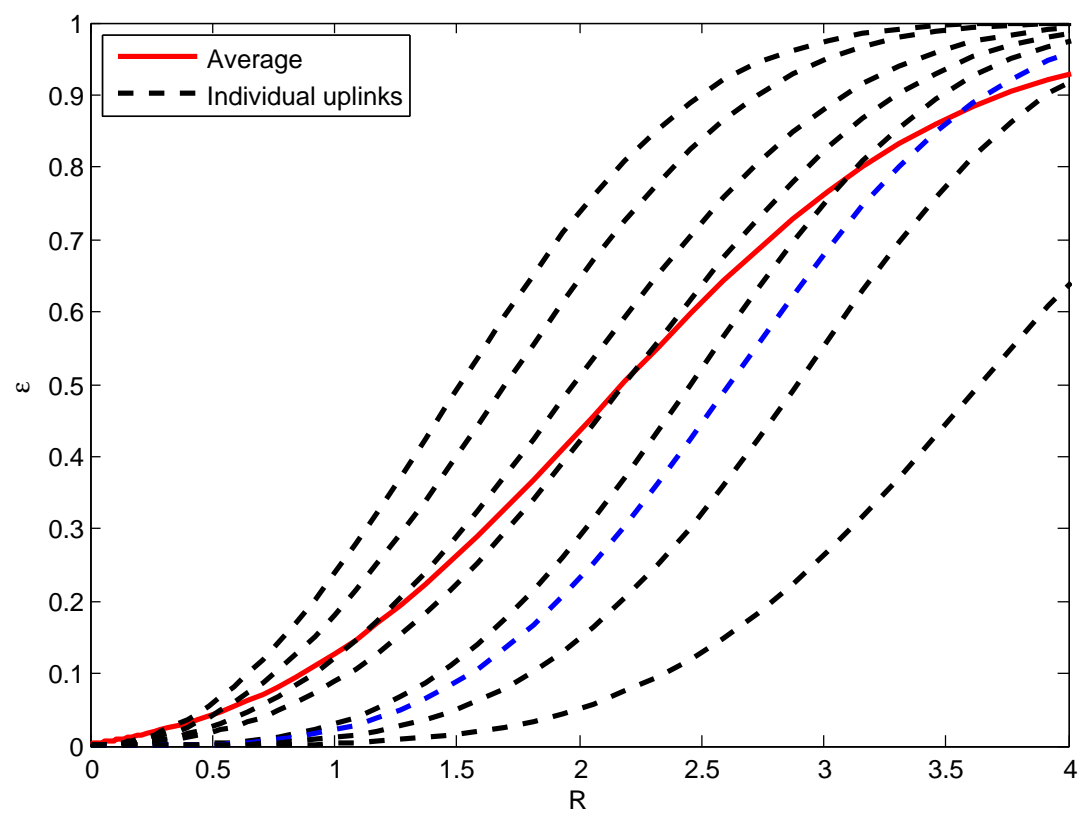

Figure 3.3: Outage probability of eight randomly selected uplinks (dashed lines) along with the average outage probability for the entire network (solid line). The results are for a halfloaded network $(M / K=G / 2)$ with distance-dependent fading and shadowing $\left(\sigma_{s}=8 \mathrm{~dB}\right)$ and are shown as a function of the rate $R$.

capacity-approaching code, two-dimensional signaling over an AWGN channel, and Gaussian interference, the SINR threshold corresponding to rate $R$ is $\beta=2^{R}-1$. The dashed lines in Fig. 3.3 were generated by selecting eight random uplinks and computing the outage probability for each using this threshold. Despite the power control, there is considerable variability in the outage probability. The outage probabilities $\left\{\epsilon_{i}\right\}$ were computed for all $M$ uplinks in the system, and the average outage probability,

$$
\mathbb{E}[\epsilon]=\frac{1}{M} \sum_{i=1}^{M} \epsilon_{i}
$$

is displayed as a solid line in the figure.

Fig. 3.4 shows the throughput as a function of rate, where the throughput of the $i^{\text {th }}$ uplink is found as

$$
\mathrm{T}_{i}=R_{i}\left(1-\epsilon_{i}\right)
$$

and represents the rate of successful transmissions. The parameters are the same as those used to produce Fig. 3.3 , and again the SINR threshold corresponding to rate $R$ is $\beta=2^{R}-1$. The plot shows the throughput for the same eight uplinks whose outages were shown in Fig. 


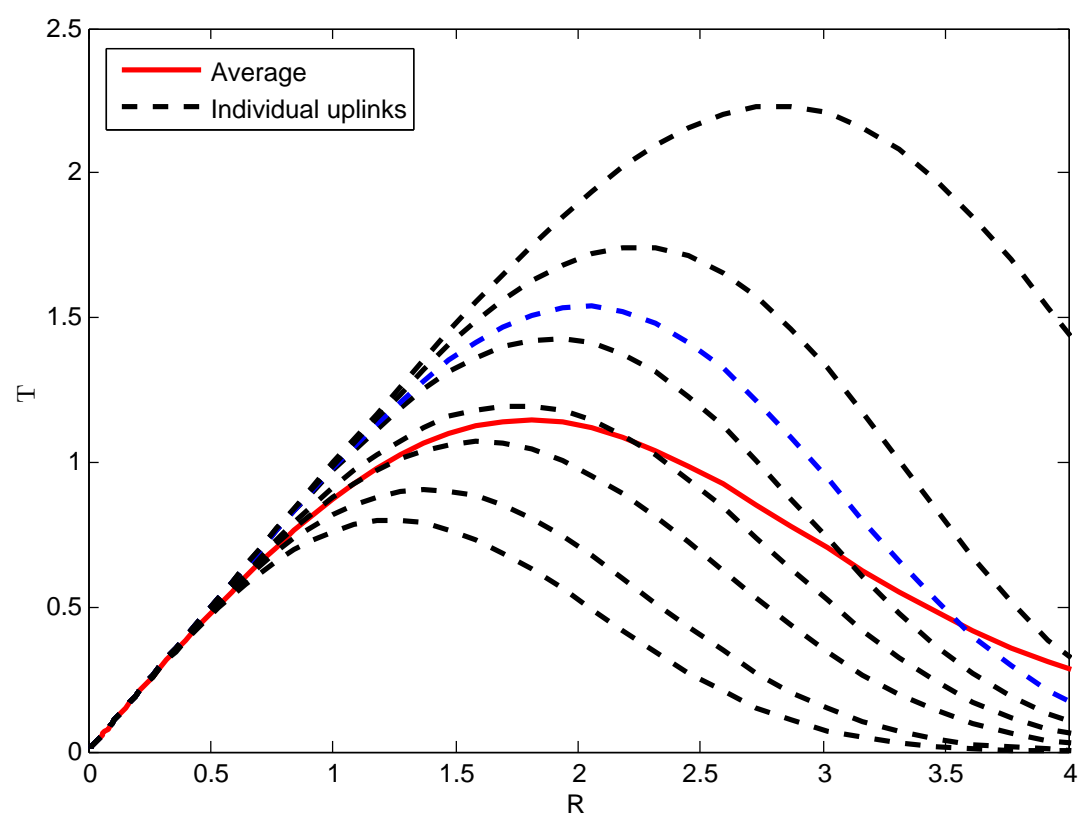

Figure 3.4: Throughput of eight randomly selected uplinks (dashed lines) along with the average throughput for the entire network (solid line). System parameters are the same used to generate Fig. 3.3 .

3.3 , as well as the average throughput

$$
\mathbb{E}[\mathrm{T}]=\frac{1}{M} \sum_{i=1}^{M} R_{i}\left(1-\epsilon_{i}\right) .
$$

\subsubsection{Fixed-Rate Policies}

A fixed-rate policy requires that all uplinks in the system must use the same rate; i.e., $R_{i}=R$ for all uplinks. On the one hand, the rate could be selected to maximize the average throughput. With respect to the example shown in Fig. 3.4, this corresponds to selecting the $R$ that maximizes the solid curve, which occurs at $R=1.81$. However, at the rate that maximizes throughput, the corresponding outage probability could be unacceptably high. When $R=1.81$ in the example, the corresponding average outage probability is $\mathbb{E}[\epsilon]=0.37$, which is too high for many applications. As an alternative to maximizing throughput, the rate $R$ could be selected to satisfy an outage constraint $\hat{\epsilon}_{\text {channel }}$ so that $\mathbb{E}[\epsilon] \leq \hat{\epsilon}_{\text {channel }}$. For instance, setting $R=0.84$ in the example satisfies an average outage constraint $\hat{\epsilon}_{\text {channel }}=0.1$ with equality. To distinguish between the two fixed-rate policies, the first policy is called maximal-throughput fixed rate (MTFR) and the second policy is called outage-constrained 
fixed rate (OCFR).

\subsubsection{Variable-Rate Policies}

If $R$ is selected to satisfy an average outage constraint, the outage probability of the individual uplinks will vary around this average. Furthermore, selecting $R$ to maximize the average throughput does not generally maximize the throughput of the individual uplinks. These issues can be alleviated by selecting the rates $R_{i}$ independently for the different uplinks. The rates could be selected to require all uplinks to satisfy the outage constraint $\hat{\epsilon}_{\text {channel }}$; i.e., $\epsilon_{i} \leq \hat{\epsilon}_{\text {channel }}$ for all $i$. This is called the outage-constrained variable-rate (OCVR policy. Alternatively, the selection could be made to maximize the throughput of each uplink; i.e., $R_{i}=\arg \max T_{i}$ for each uplink, where the maximization is over all possible rates. This is called the maximal-throughput variable-rate (MTVR) policy. Both policies can be implemented by having the BS track the outage probabilities or throughputs of each uplink and feeding back rate-control commands to ensure that the target performance is achieved. The outage probability can be easily found by encoding the data with a cyclic redundancy check (CRC) code and declaring an outage when a check fails.

It is much simpler to adapt the chip rate, code rate, or information rate than to implement modulators and demodulators that can be adapted. Adapting the chip rate is generally undesirable because that would require a change in bandwidth. Thus, for both variablerate policies, it is assumed that the code rate is adapted by maintaining the duration of channel symbols while varying the number of information bits per symbol. The spreading factor $G$ and symbol rate are held constant, so there is no change in bandwidth. However, a major drawback with rate control is that the rates required to maintain a specified outage probability varies significantly among the UE in the network. This variation results in low throughput for some $\overline{\mathrm{UE}}$, particularly those located at the edges of the cells, while other UEs have a high throughput. Unequal throughputs may not be acceptable when a UE may be stuck or parked near a cell edge for a long time, and an interior UE may have more allocated throughput than it needs.

Due to the use of power control, the common intercell interference, and the lack of 
intracell interference, the average received SINR for all UEs in a sector will generally be identical. When all uplinks have the same Nakagami-m factor, then the conditional outage probabilities of all uplinks in the same sector will be identical. Thus, under rate control, all links in the same sector are generally assigned the same rate. However, in the case that the Nakagami-m factor is not the same for all links, such as in the distance-dependent fading model, the outage probability will vary over the links, and different rates will be assigned to the uplinks of the cell sector.

\subsection{Cell Reselection}

Because the number of orthogonal spreading sequences available to the cell is limited to $G$, the number of served $\mathrm{UE} ;$ is limited to $\mathcal{M}_{j} \leq G$. If there are $\mathcal{M}_{j}>G$ UE for which $\mathrm{g}(i)=j$ according to 3.1 , then some of these UEs will either be refused service by $X_{i}$ or given service at a lower rate (through the use of an additional time multiplexing). In the following, two policies are considered for handling this situation. With the first policy, which is called denial due to overload (DDO), the $\mathcal{M}_{j}-G$ UE; whose path losses from the BS are greatest are denied service, in which case they do not appear in the set $\mathcal{Y}_{j}$ for any $j$, and their rates are set to $R_{i}=0$. With the second policy, which is called reselection due to overload (RDO), each of these $\mathcal{M}_{j}-G \overline{\mathrm{UE}}$ in an overloaded cell attempts to connect to the $\mathrm{BS}$ with the next-highest path loss out to a maximum reassociation distance $r_{\max }$. If no available cell is found within distance $r_{\max }$, the rate is set to $R_{i}=0$, and the UE is denied service. Note that the denial policy is the same as the reselection policy with $r_{\max }=0$.

\subsection{Performance Analysis}

Let $\mathbb{E}_{\boldsymbol{\Omega}}[R]$ represent the mean value of the variable $R$, which can be found by numerically averaging the values of $R$ obtained using the procedure described above. While $\mathbb{E}_{\boldsymbol{\Omega}}[R]$ is a useful metric, it does not account for the loss in throughput due to the inability to successfully decode during an outage, and it does not account for the spatial density of transmissions. These considerations are taken into account by the area spectral efficiency (ASE), which can 
be expressed as 52,75

$$
\overline{\mathcal{S}}_{\text {ase }}=\lambda\left(1-\hat{\epsilon}_{\text {channel }}\right) \mathbb{E}_{\boldsymbol{\Omega}}[R]
$$

where $\lambda=K /|\mathcal{A}|$ is the density of transmissions in the network. ASE can be interpreted as the spatial intensity of transmissions; i.e., the rate of successful data transmission per unit area. The ASE is obtained by using a Monte Carlo approach as follows:

1. Draw a realization of the network is obtained by placing $K \overline{\mathrm{BSS}}$ and $M$ UE $;$ within a finite area $\mathcal{A}$ according to the uniform-clustering model with minimum $\mathrm{BS}$ separation $r_{\text {exbs }}$ and minimum UE separation $r_{\text {ex }}$.

2. Compute the path loss from each $\overline{\mathrm{BS}}$ to each UE by applying randomly generated shadowing factors, if shadowing is present.

3. Determine the set of UE associated with each cell sector, applying the reselection policy if one is used.

4. At each sector antenna, the power-control policy is applied to determine the power the antenna receives from each $\mathrm{UE}$ that it serves.

5. In each cell sector, the rate-control policy is applied to determine the rate and SINR threshold.

6. For each uplink, the outage probability is computed by applying the rate-control policy and using 3.2 in $(2.9),(3.4)$, and 3.6 .

7. Equation (3.8) is applied to calculate the average outage probability for the network realization. Equation (3.9) is applied to calculate the throughputs according to the resource-allocation policy, and then (3.10) is applied to compute the average throughput for the network realization.

8. Finally, multiplying by the density $\lambda$ and averaging over all simulated network realizations, it is computed the average rate $\mathbb{E}_{\boldsymbol{\Omega}}[R]$, the average outage probability $\mathbb{E}_{\boldsymbol{\Omega}}[\epsilon]$ and the average ASE $\overline{\mathcal{S}}_{\text {ase }}$. 


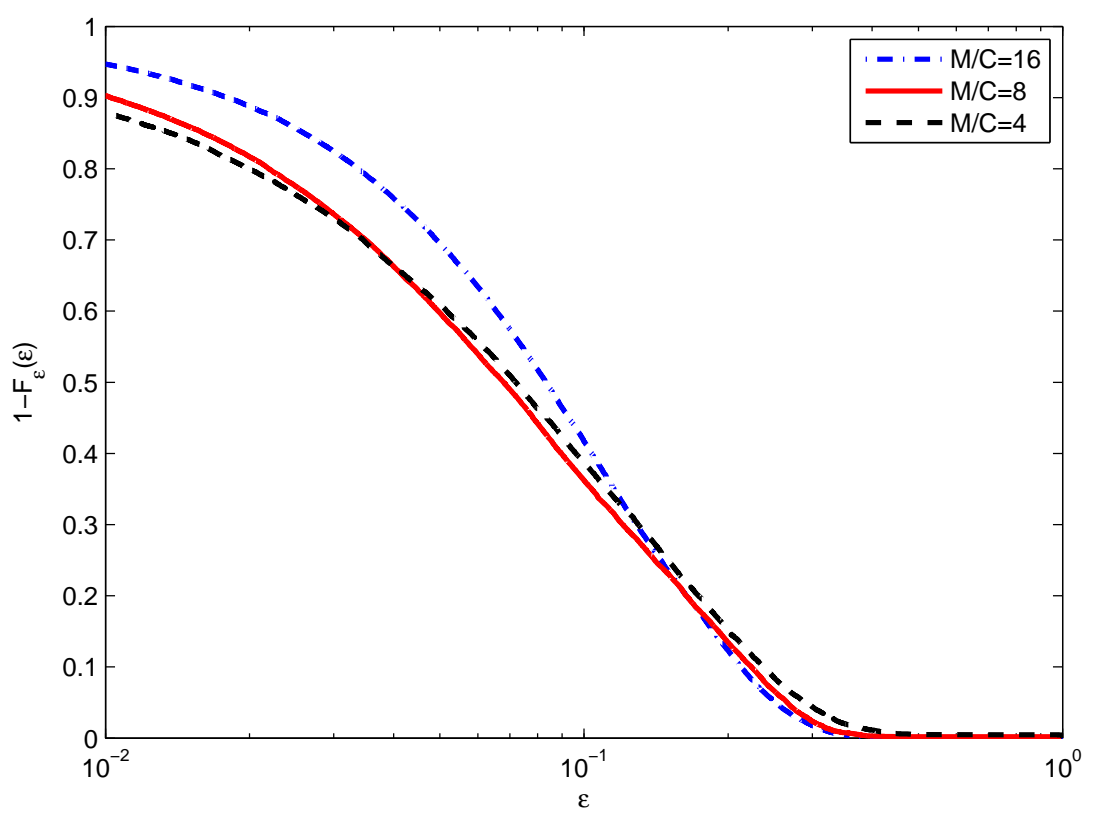

Figure 3.5: CCDF of the outage probability using an OCFR policy, $R=2$, three network loads, distance-dependent fading, and shadowing.

In the following, the Monte Carlo method is performed over $\Upsilon=1,000$ network topologies. Two fading models are considered: Rayleigh fading, where $m_{i, j}=1$ for all $i$, and distance-dependent fading given by (3.7), where $r_{\text {los }}=r_{\text {ex }}$. Both unshadowed and shadowed $\left(\sigma_{s}=8 \mathrm{~dB}\right)$ environments are considered. For what concerns the cell reselection policy, DDO is used $\left(r_{\max }=0\right)$, if not otherwise stated.

\subsubsection{Outage-Constrained Fixed-Rate Policy}

As illustrated in Fig. 3.3, using an OCFR policy results in a high variability of outage probabilities. Fig. 3.5illustrates the variability of $\epsilon$ with respect to all uplinks and simulation trials under a OCFR policy by plotting its complementary cumulative distribution function (CCDF) with $R=2$, three network loads, distance-dependent fading, and shadowing.

\subsubsection{Outage-Constrained Variable-Rate Policy}

With the OCVR policy, the rate $R_{i}$ (equivalently, $\beta_{i}$ ) of each uplink is selected such that the outage probability does not exceed $\hat{\epsilon}_{\text {channel }}=0.1$. While the outage probability is fixed, the rates of the uplinks will be variable. In Fig. 3.6, the average rate is shown as a function 


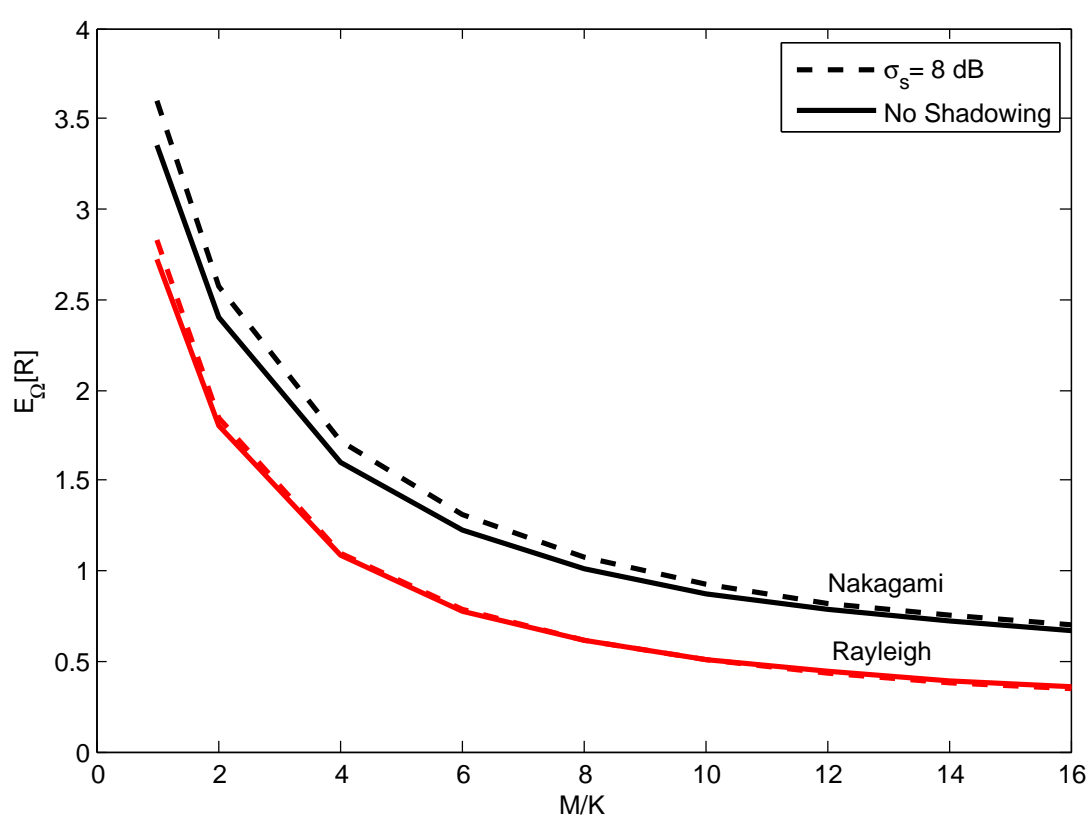

Figure 3.6: Average rate of the OCVR policy as function of the load $M / K$ for both Rayleigh and distance-dependent fading, and both shadowed $\left(\sigma_{s}=8 \mathrm{~dB}\right)$ and unshadowed cases.

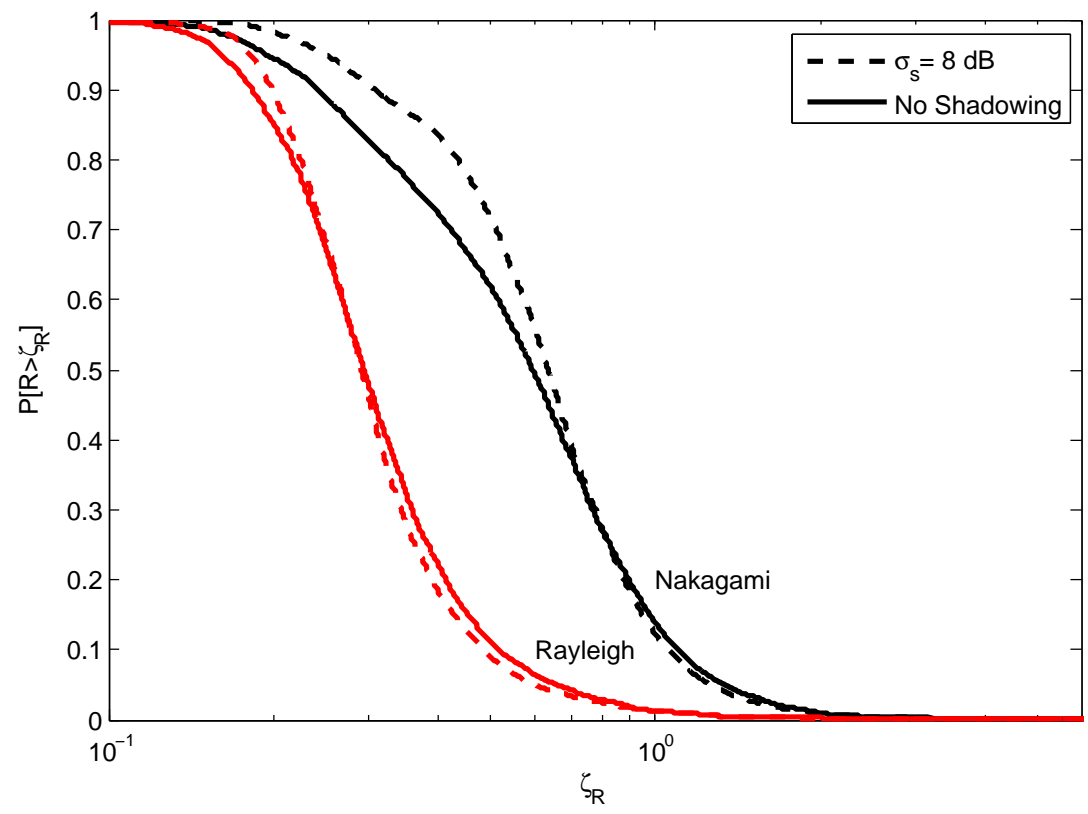

Figure 3.7: CCDF of the rate for fully-loaded system $(M / K=G)$ under the OCVR policy in Rayleigh and distance-dependent fading, and both shadowed $\left(\sigma_{s}=8 \mathrm{~dB}\right)$ and unshadowed cases. 


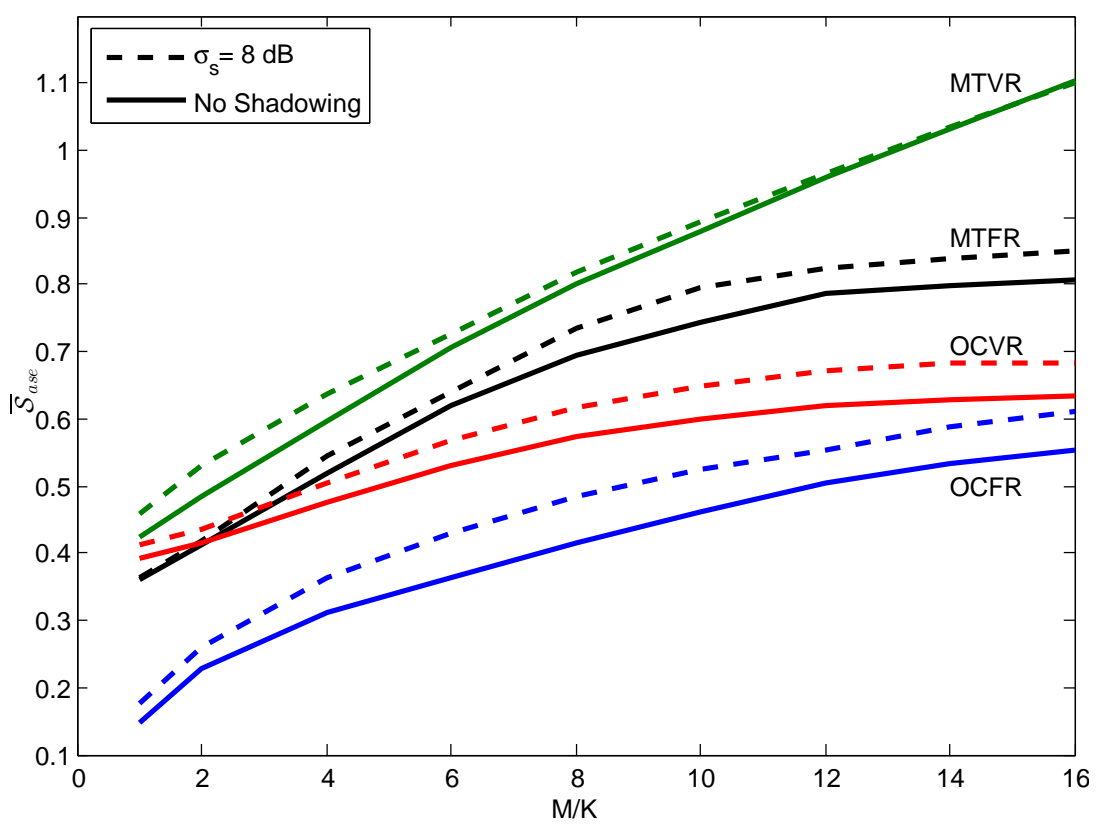

Figure 3.8: ASE for the four network policies as function of the load $M / K$ for distancedependent fading and both shadowed $\left(\sigma_{s}=8 \mathrm{~dB}\right)$ and unshadowed cases.

of the load $M / K$. In Fig. 3.7, the variability of $R_{i}$ is illustrated by showing the CCDF of the rate for a fully-loaded system $(M / K=G=16)$ in both Rayleigh fading and distancedependent fading, and both with and without shadowing. The fairness of the system can be determined from this figure, which shows the percentage of uplinks that meet a particular rate requirement.

\subsubsection{Policy Comparison}

Fig. 3.8 shows the average $\mathrm{ASE} \overline{\mathcal{S}}_{\text {ase }}$ of the four network policies in distance-dependent fading, both with and without shadowing, as a function of the load $M / K$. While the ASEs of the MTFR and MTVR policies are potentially superior to those of the OCFR and OCVR policies, this advantage comes at the cost of a variable and high value of $\epsilon$, which is generally too large for most applications. The OCVR policy has a higher average ASE than the OCFR policy. 


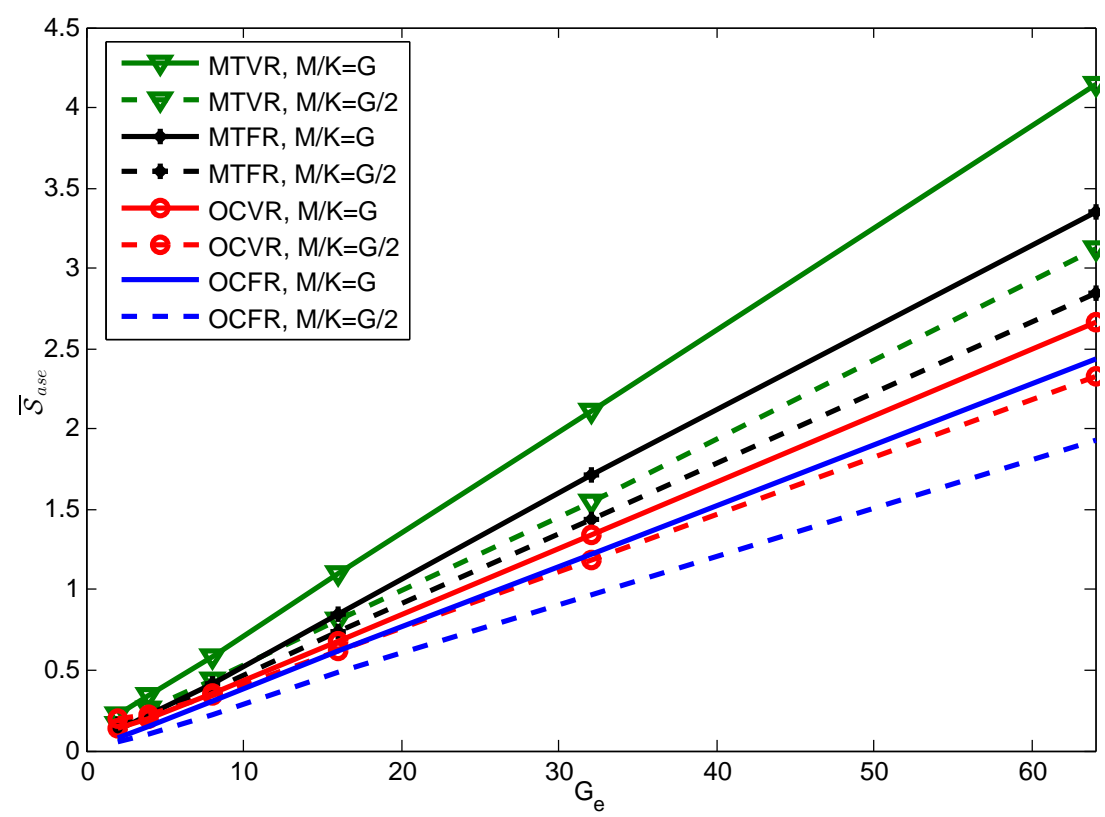

Figure 3.9: ASE as function of effective spreading factor $G_{e}$ for two values of system load, distance-dependent fading, and shadowing with $\sigma_{s}=8 \mathrm{~dB}$.

\subsubsection{Spreading factor}

Fig. 3.9 shows the ASE $\overline{\mathcal{S}}_{\text {ase }}$ as a function of the effective spreading factor $G_{e}$ for the shadowed distance-dependent-fading channel. Two loads are shown for each of the four policies. An increase in $G_{e}$ is beneficial for all policies, but the MTVR policy benefits the most.

\subsubsection{Base-station Exclusion Zone}

Fig. 3.10 shows the ASE $\overline{\mathcal{S}}_{\text {ase }}$ for each of the four policies as a function of the BS exclusion-zone radius $r_{\text {ex }_{\text {bs }}}$ for $M / K=G / 2$ and two values of path loss exponent $\alpha$. The distance-dependent fading model is used, and shadowing is applied with $\sigma_{s}=8 \mathrm{~dB}$. The two policies that constrain the outage probability are more sensitive to the value of $r_{\text {ex }_{\mathrm{bs}}}$ than the two policies that maximize throughput. An increase in $\alpha$ increases $\overline{\mathcal{S}}_{\text {ase }}$ for all four policies.

\subsubsection{Cell Association}

In the previously shown results of this section, UE; were denied service if they were in an overloaded sector. By allowing $\mathrm{UE} ;$ to reselect $\mathrm{BS} ;$ out to a distance of $r_{\max }$, the probability 


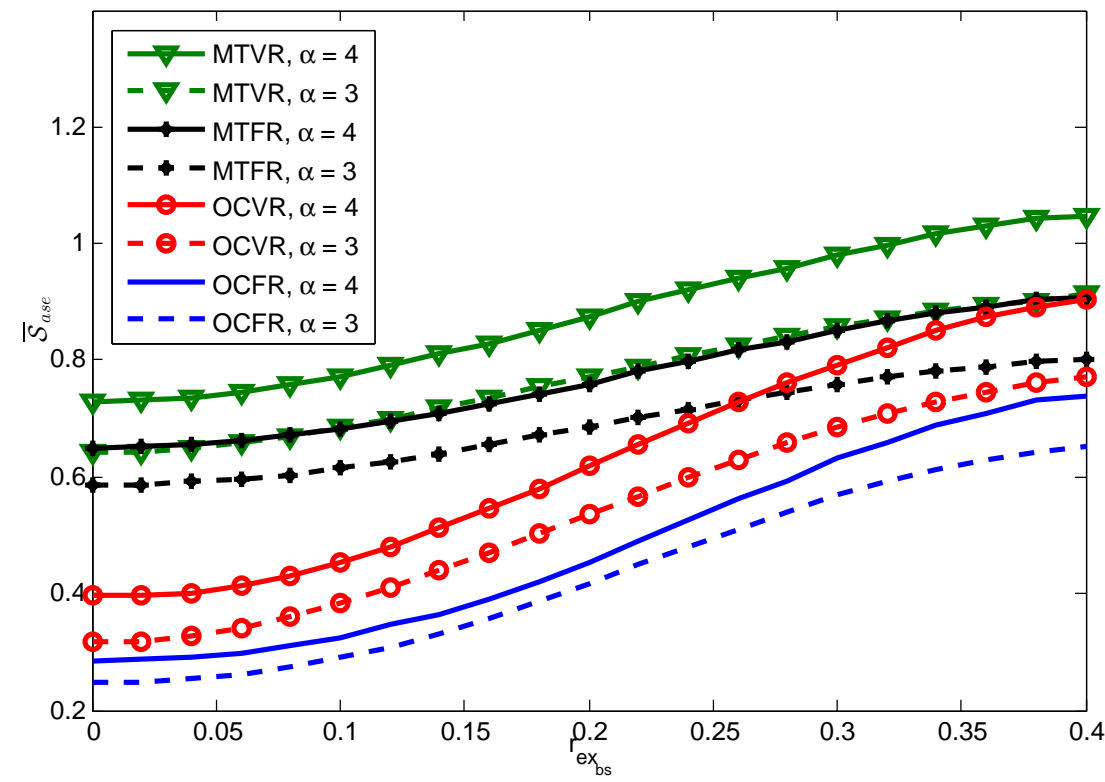

Figure 3.10: ASE as a function of the BS exclusion-zone radius $r_{\mathrm{ex}_{\mathrm{bs}}}$ for four policies and two values of path loss exponent $\alpha$.

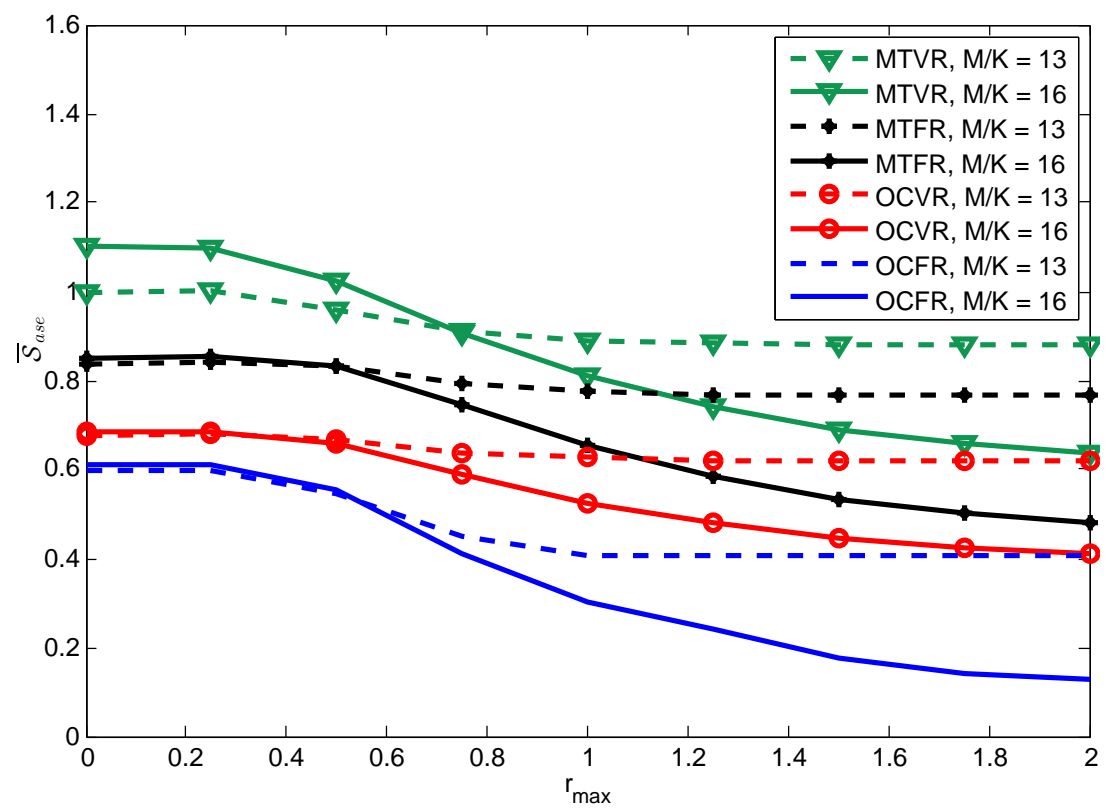

Figure 3.11: ASE as a function of the maximum reselection distance $r_{\max }$. 
that they are denied service due to overload can be reduced. By setting $r_{\max }$ sufficiently large, the probability that a $\mathrm{UE}$ is denied service due to overload can be made arbitrarily small. The tradeoff for keeping these UEs connected is that $\overline{\mathcal{S}}_{\text {ase }}$ decreases because UEs must transmit at a higher power, thereby causing more interference. This tradeoff is illustrated in Fig. 3.11, which shows the ASE as a function of $r_{\max }$ for all four policies and two system loads. In general, $\overline{\mathcal{S}}_{\text {ase }}$ decreases with increasing $r_{\max }$, and the decrease is more pronounced for the more heavily loaded system.

\subsection{Chapter Summary}

This chapter presented a new approach for modeling and characterizing the statistical performance of a conventional cellular network based on the analytical framework provided in Chap. 2. The proposed analytical approach provides a way to compare various resource allocation and cell association techniques and study a cellular network in a more accurate way, by discharging some of the unfeasible assumptions usually made. In this chapter, the performance analysis for the uplink of a DS-CDMA system is provided in terms of ASE, and several different resource allocations are considered. The performance analysis has emphasized the key effect of the minimum separation among base stations and the spreading factor. 


\section{Chapter 4}

\section{Diversity Combining: Outage Analysis and Applications}

Spatial diversity can be achieved through cooperative transmissions among UE an appropriate combining of the signals at the destination. One technique, which is used in many modern wireless communication systems, is diversity combining [76], where the receiver combines multiple received signals in order to improve the quality of the decoding.

Nowadays, diversity combining is used in several wireless networks and transmission modes, and while this diversity technique is widely known, the fundamental limits of the networks that use it remain largely unknown due to the lack of an accurate and yet tractable network model. This chapter develops an analytical framework to evaluate the channel outage probability conditioned on the network geometry in a Nakagami faded channel when signals arriving over different paths are diversity combined at the receiver in the presence of noise and interference. The analysis is a generalization of the framework described in Chap. 2, and is applied to study two different cooperative cellular networks, which are both modeled using a constrained spatial model: DS-CDMA multi-cell cooperation (MCC) downlink and an multicast-broadcast single-frequency network (MBSFN) network. For both applications, an accurate model is built and a performance analysis is performed, emphasizing how cooperative communications should be used to maximize their benefits. In particular, insights are provided on how the cell edge should be defined and how inter-symbol interference (ISI) effects performance. 


\subsection{Network Model}

Consider a network comprising $M$ transmitters $\left\{X_{1}, \ldots, X_{M}\right\}$ and a reference receiver $Y_{j}$. Let $\mathcal{X}_{j}$ be the set of transmitters, which in a tightly synchronized manner transmit a reference signal to the receiver, and let $\mathcal{N}_{j}=\left|\mathcal{X}_{j}\right|$ denote their number. It is assumed that the receiver at $\mathrm{UE} Y_{j}$ can combine all the resolvable multipath components from the set of $\mathcal{X}_{j}$. This is a valid assumption if at the receiver is used an appropriate equalizer (for instance, for a DS-CDMA system, a rake receiver can be equipped at the UE). Furthermore, let $\mathcal{G}_{j}$ be the set of the indexes of the transmitters serving $Y_{j}$. In this case, the instantaneous SINR $\gamma_{j}$ at the receiving $\mathrm{UE} X_{j}$ is the sum of the SINR from each of the $N_{j}$ serving transmitters 74,77]:

$$
\gamma_{j}=\sum_{k \in \mathcal{G}_{j}} \gamma_{k, j}
$$

where $\gamma_{k, j}$ is the SINR of the signal received from transmitter $X_{k}$ (i.e., the SINR in the $k^{t h}$ branch) at the $\mathrm{UE} Y_{j}$, and by using the same notation as in Chap. 2 it can be written as

$$
\gamma_{k, j}=\frac{\rho_{k, j}}{\mathcal{N}+\sum_{i \notin \mathcal{G}_{j}} I_{i} \rho_{i, j}} .
$$

A commonly accepted approach to analyzing diversity combining is to assume that the interference realizations in the different branches are the same; i.e., the interference is fullycorrelated among the branches (cf., 78 84). While strictly speaking, this condition is not always met, the assumption makes the analysis manageable. Furthermore, as a worst-case scenario, the analysis makes it possible to obtain an upper bound on outage probability, which happens to be tight [85, 86]. Under this assumption, the instantaneous SINR at UE $Y_{j}$ is

$$
\gamma_{j}=\frac{N_{j} \sum_{i \in \mathcal{G}_{j}} g_{i, j} \Omega_{i, j}}{\mathrm{SNR}^{-1}+\sum_{i \notin \mathcal{G}_{j}} I_{i} g_{i, j} \Omega_{i, j}}
$$

where SNR $=d_{0}^{\alpha} P_{0} / \mathcal{N}$ and

$$
\Omega_{i, j}= \begin{cases}10^{\xi_{i, j} / 10} r_{i, j}^{-\alpha} & i \in \mathcal{G}_{j} \\ \frac{P_{i, j}}{P_{0}} 10^{\xi_{i, j} / 10} r_{i, j}^{-\alpha} & i \notin \mathcal{G}_{j}\end{cases}
$$




\subsection{Conditional Outage Probability}

Similar to Sec. 2.2, conditioning on $\boldsymbol{\Omega}$, the outage probability of UE $Y_{j}$ is

$$
\epsilon_{j}(\Omega)=P\left[\gamma_{j} \leq \beta \mid \Omega\right]
$$

By defining

$$
\begin{array}{r}
\mathrm{S}_{j}=\sum_{i \in \mathcal{G}_{j}} \beta^{-1} g_{i, j} \Omega_{i, j} \quad, \quad Y_{i, j}=I_{i} g_{i, j} \Omega_{i, j} \\
\mathrm{Z}_{j}=\mathrm{S}_{j}-\sum_{i \notin \mathcal{G}_{j}} Y_{i, j}
\end{array}
$$

the conditional outage probability may be expressed as

$$
\epsilon_{j}(\Omega)=P\left[\mathrm{Z}_{j} \leq \mathrm{SNR}^{-1} \mid \Omega\right]=F_{\mathrm{Z}_{j}}\left(\mathrm{SNR}^{-1} \mid \Omega\right)
$$

which is the $\mathrm{CDF}$ of $\mathrm{Z}_{j}$ conditioned on $\boldsymbol{\Omega}_{j}$ and evaluated at $\mathrm{SNR}^{-1}$.

Theorem 4.2.1 The conditional outage probability is 87]

$$
\begin{aligned}
\epsilon_{j}(\boldsymbol{\Omega})= & \sum_{k \in \mathcal{G}_{j}} \sum_{n=1}^{m_{k, j}} \Xi_{N_{j}}\left(k, n,\left\{m_{q, j}\right\}_{\forall q \in \mathcal{G}_{j}},\left\{\frac{\Omega_{q, j}}{\beta m_{q, j}}\right\}_{\forall q \in \mathcal{G}_{j}}\right) \\
& \left\{1-\exp \left(-\frac{\beta m_{k, j}}{\Omega_{k, j} \mathrm{SNR}}\right) \sum_{\mu=0}^{n-1}\left(\frac{\beta m_{k, j}}{\Omega_{k, j} \mathrm{SNR}}\right)^{\mu} \sum_{t=0}^{\mu} \frac{\mathrm{SNR}^{t}}{(\mu-t) !} \sum_{\substack{\ell_{i} \geq 0 \\
\sum_{i=0}^{M-\mathcal{N}_{j}} \ell_{i}=t}}\right. \\
& \left.\prod_{i \notin \mathcal{G}_{j}}\left[\left(1-p_{i}\right) \delta_{\ell_{i}}+\frac{p_{i} \Gamma\left(\ell_{i}+m_{i, j}\right)}{\ell_{i} ! \Gamma\left(m_{i, j}\right)}\left(\frac{\Omega_{i, j}}{m_{i, j}}\right)^{\ell_{i}}\left(\frac{\beta m_{k, j}}{\Omega_{k, j}} \frac{\Omega_{i, j}}{m_{i, j}}+1\right)^{-\left(m_{i, j}+\ell_{i}\right)}\right]\right\}
\end{aligned}
$$

where the function $\Xi_{L}\left(k, n,\left\{r_{q}\right\}_{q=1}^{L},\left\{\eta_{q}\right\}_{q=1}^{L}\right)$ is given by (D.2).

Proof: The details of the derivation are given in Appendix $D$.

Notice that (4.9) reduces to 2.9$)$ when there is only one transmitter $\left(\mathcal{N}_{j}=1\right)$. Furthermore, following the approach described in Chap. 2 and similar analytical steps, it is possible to evaluate the spatially averaged outage probability for diversity combining receivers. 


\subsection{Applications}

In this section, the previously developed analytical framework is applied to two cases of study. First, it is used to analyze the performance of an DS-CDMA] handover) for different resource allocation policies, and to provide insight regarding how the cell edge should be defined for this type of network in order to maximize the throughput. Then, it is applied to study an LTE broadcasting transmission mode, called MBSFN, and point out the tradeoff between the number of cooperative transmissions and ISI].

\subsubsection{Multi-Cell Downlink Cooperation}

One of the main factors limiting the throughput in cellular networks is intercell interference. A promising approach for mitigating such interference is $\mathrm{MCC}$, which involves the joint processing of signals transmitted to multiple $\mathrm{BS}$; and/or received by them $77,88,89$. In practice, multi-cell cooperation is made possible by the presence of an infrastructure of backhaul links connecting individual BS; to a central processor or to one another [90], which allow $\mathrm{BS}$ s to exchange data and channel state information (CSI). For instance, the LTE-A cellular standard can implement such backhaul links through the X2 interface [91]. Thereby, cells can be grouped into finite clusters and $\overline{\mathrm{BS}}$; in a same cluster cooperate to decouple the assigned UE; 92 94].

In this sub-section, the downlink signals use orthogonalDS-CDMA sequences with a common spreading factor $G$. A rake receiver is used at each $\mathrm{UE}$, and the signals from the serving BSs are assumed to be separated in time by at least a chip period, denoted by $T_{c}$. Because the sequences transmitted by a particular BS are orthogonal, the only source of intracell interference is due to the unresolvable multipath components and the corresponding loss of orthogonality. However, if the ratio of the maximum-power and minimum-power unresolvable multipath components is sufficiently small, then the unresolvable multipath components will have negligible effect. For this reason, the intracell interference is neglected and it is assumed that the intercell interference is the only source of interference. An asynchronous quadriphase direct-sequence systems is considered. Assume the networks is composed of $M$ BSs $\left\{X_{i}, \ldots, X_{M}\right\}$ and $K \widehat{\mathrm{UE}}\left\{Y_{i}, \ldots, Y_{K}\right\}$ placed in an area $\mathcal{A}$. In this case the normalized 


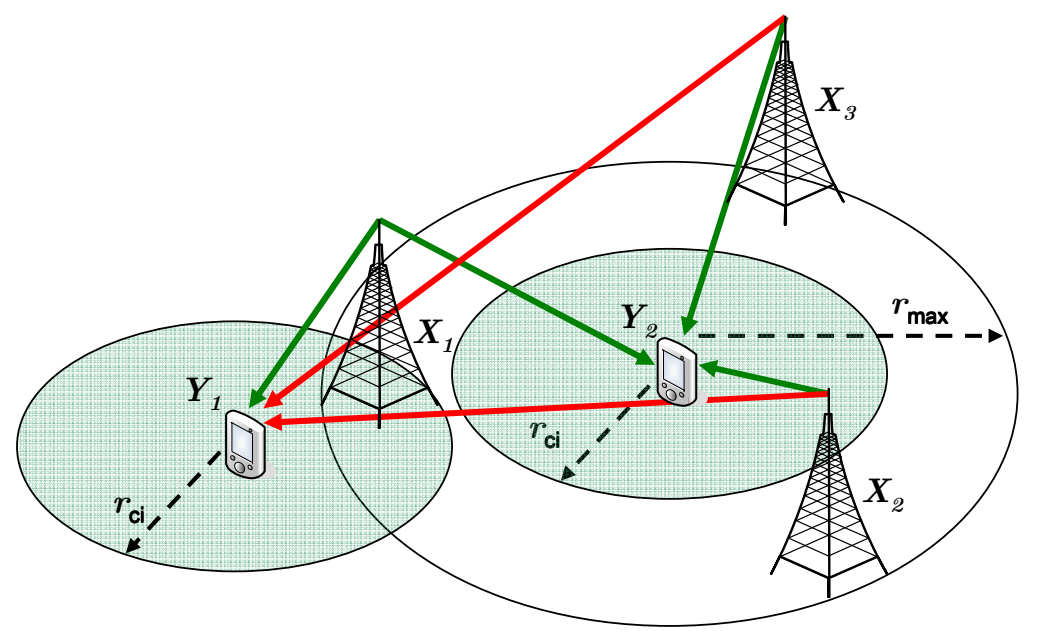

Figure 4.1: Illustration of the cell association: UE $Y_{1}$ is only served by BS $X_{1}$, since $X_{1}$ is located at effective distance lower than $r_{\mathrm{ci}}$; UE $Y_{2}$ is instead served by a plurality of BSs within a maximum effective distance $r_{\max }$, since there is no BSs at an effective distance lower than $r_{\mathrm{ci}}$.

received power at the $\mathrm{UE} Y_{j}$, when the $\mathrm{BS} X_{i}$ transmits, is

$$
\Omega_{i, j}= \begin{cases}10^{\xi_{i, j} / 10} r_{i, j}^{-\alpha} & i \in \mathcal{G}_{j} \\ \frac{h P_{i, j}}{G P_{0}} 10^{\xi_{i, j} / 10} r_{i, j}^{-\alpha} & i \notin \mathcal{G}_{j}\end{cases}
$$

and the conditioned outage probability can be evaluated by substituting (4.10) in 4.9).

\section{Cell Association}

Let define the effective distance as

$$
\tilde{r}_{i, j}=10^{-\xi_{i, j} /(10 \alpha)} r_{i, j}
$$

which is the distance perturbed by the shadowing. In the absence of shadowing, $r_{i, j}=\tilde{r}_{i, j}$. Let $r_{\mathrm{ci}}$ represent the radius of the cell interior. Any UE that is within an effective distance $r_{\mathrm{ci}}$ of a $\mathrm{BS}$ will be served by just that $\overline{\mathrm{BS}}$, and $\mathrm{UE}$ beyond $r_{\mathrm{ci}}$ will attempt to connect to a plurality of $\overline{\mathrm{BSS}}$; out to some maximum distance $r_{\max }$, where $r_{\mathrm{ci}} \leq r_{\max }$, as illustrated by Fig. 4.1. It follows that $\mathrm{UE} Y_{j}$ is served by those $\mathrm{BS} X_{i}$ whose indices are in the set

$$
\mathcal{G}_{j}= \begin{cases}\underset{i}{\operatorname{argmin}}\left\{\tilde{r}_{i, j}\right\} & \text { if } \min _{i}\left(\tilde{r}_{i, j}\right) \leq r_{\mathrm{ci}} \\ \left\{i: \tilde{r}_{i, j}<r_{\text {max }}\right\} & \text { if } \min _{i}\left(\tilde{r}_{i, j}\right)>r_{\mathrm{ci}}\end{cases}
$$


Let $\mathcal{Y}_{i}$ be the set of $\mathrm{UE}$; connected to $\mathrm{BS} X_{i}$, and $K_{i}=\left|\mathcal{Y}_{i}\right|$ be the number of $\mathrm{UE}$ s served by $X_{i}$. Because there are only $G$ orthogonal spreading sequences per cell, the number of $\overline{\mathrm{UE}}$ connected to $X_{i}$ is limited to $K_{i} \leq G$. In the following, if there are $K_{i}>G$ UEs a DDO policy is adopted, which is described in Sec. (3.5). In particular, in this case it is assumed that the first $G$ UE whose effective distances are closest to $X_{i}$ are served, while the others are refused service and marked as having zero rate.

\section{Power allocation}

It is assumed that the $\overline{\mathrm{BS}}$ s transmit with a common power $P_{0}$ such that

$$
\frac{1}{1-f_{p}} \sum_{j: Y_{j} \in \mathcal{Y}_{i}} P_{i, j}=P_{0}
$$

for each $\mathrm{BS}$, where $f_{p}$ is the fraction of the $\mathrm{BS}$ power reserved for pilot signals needed for synchronization and channel estimation.

$\mathrm{BS} X_{i}$ allocates the power transmitted to $\mathrm{UE} Y_{j}$ according to the fractional power-control policy

$$
P_{i, j}=P_{0}\left(1-f_{p}\right)\left[\frac{1-f_{\mathrm{fpc}}}{K_{i}}+f_{\mathrm{fpc}} \tilde{r}_{i, j}^{\alpha}\left(\sum_{j: Y_{j} \in \mathcal{Y}_{i}} \tilde{r}_{i, j}^{\alpha}\right)^{-1}\right]
$$

where $0 \leq f_{\mathrm{fpc}} \leq 1$ is the fractional power-control factor. In this subsection, the two extreme situations are considered: $f_{\mathrm{fpc}}=0$, which corresponds to an equal transmit power (ETP) policy, and $f_{\mathrm{fpc}}=1$, which corresponds to an equal received power (ERP) policy. In particular, when $f_{\mathrm{fpc}}=0, P_{0}$ is allocated with an equal-share policy, which involves $\mathrm{BS}$ $X_{i}, i \in \mathcal{G}_{j}$, transmitting to $\mathrm{UE} Y_{j}$ with the same power, while when $f_{\mathrm{fpc}}=1$ every $\mathrm{UE}$ connected to a particular $\mathrm{BS} X_{i}$ has the same received power.

\section{Rate allocation}

Under the given power-allocation policy, the rate allocated to each user is selected to satisfy an outage probability. In particular, the threshold $\beta_{j}$ of $\mathrm{UE} Y_{j}$ is selected such that the outage probability of $\mathrm{UE} Y_{j}$ satisfies the constraint $\epsilon_{j} \leq \hat{\epsilon}_{\text {channel }}$. Since the SINR threshold $\beta_{j}$ is related to the code rate $R_{j}$, this corresponds to perform link adaptation. 
In the following, it is assumed a continuum of possible rates, and the Shannon capacity for complex discrete-time AWGN channels is used to related this two entities: $R_{j}=\log _{2}\left(1+\beta_{j}\right)$. This assumption is fairly accurate for systems that use capacity-approaching codes and a large number of code rates and modulation schemes, such as modern cellular systems, which use turbo codes with a large number of available modulation and coding schemes.

\section{Performance Analysis}

Under outage constraint $\hat{\epsilon}_{\text {channel }}$, the performance of a given network realization is largely determined by the set of achieved rates $\left\{R_{j}\right\}$ of the users in the network. Similar to Chap. 3. a random spatial model is adopted, and in particular both $\mathrm{BS} ; \mathrm{s}$ and $\mathrm{UE} ;$ are distributed according with a $\overline{\mathrm{UCP}}$ with exclusion zone of radius $r_{\mathrm{ex}}$ for the $\overline{\mathrm{BS}}$ s, and exclusion zone of radius $r_{\mathrm{ex}_{\mathrm{mb}}}$ for the $\mathrm{UE}$, within a circular arena of radius $r_{\mathrm{out}}$. The distance-dependent fading model given by (3.7) is used, and it is assumed that $r_{\text {los }}=r_{\text {ex }_{\text {bs }}}$.

The statistics of $R$ can be found as follows:

1. Draw a realization of the network by placing $M[\overline{\mathrm{BS}}$ s and $K \overline{\mathrm{UE}}$ s within the disk of radius $r_{\text {out }}$ according to a $\mathrm{UCP}$.

2. Compute the path loss from each $\overline{\mathrm{BS}}$ to $\mathrm{UE}$, applying randomly generated shadowing factors if shadowing is present.

3. Determine the set of UE associated with each $\overline{\mathrm{BS}}$, applying the cell association policy.

4. At each $\mathrm{BS}$, apply the power-allocation policy to determine the power it transmits to each UE that it serves.

5. By setting the outage equal to the outage constraint, invert 4.9), once (4.10) is substituted within it, to determine the SINR threshold for each UE in the cell. By applying the Shannon capacity for complex discrete-time AWGN channels, find the rate of the UE

6. Repeat this process for a large number of network realizations, all with the same values of $\left\{K, M, r_{\text {out }}, r_{\mathrm{ex}_{\mathrm{bs}}}, r_{\mathrm{ex}_{\mathrm{mb}}}, \sigma_{s}\right\}$. 


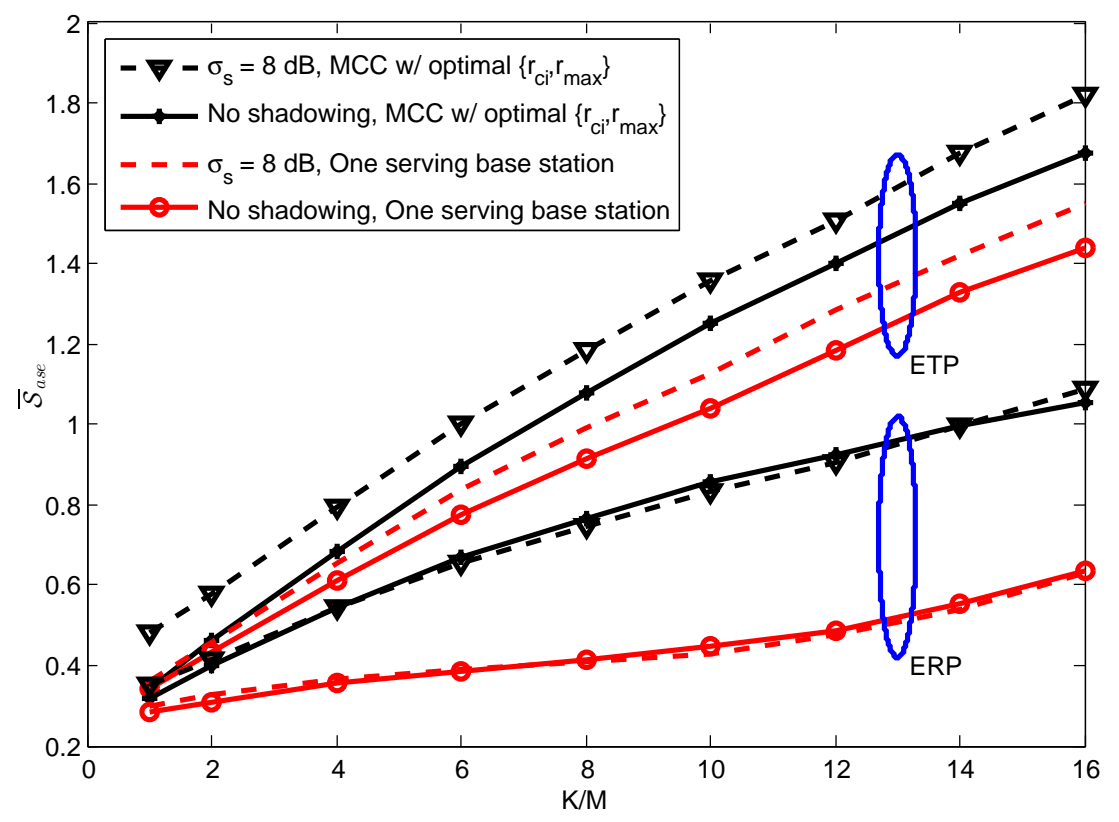

Figure 4.2: ASE as a function of $K / M$ with ERP and ETP policy, for both a shadowed $\left(\sigma_{s}=8 \mathrm{~dB}\right)$ and unshadowed environment.

The mean of the variable $R$ is then found by numerically averaging the values of $R$ obtained using the procedure described in the previous paragraph. Since $\mathbb{E}_{\boldsymbol{\Omega}}[R]$ does not account for the loss in throughput due to the inability to successfully decode during an outage, and it does not account for the spatial density of transmissions, the ASE $\overline{\mathcal{S}}_{\text {ase }}$ is used as a performance metric, which is given by (3.11).

In this subsection, it is assumed a network with $M=50$ BS of radius $r_{\text {out }}=2$ with $\mathrm{BS}$ exclusion zones of radius $r_{\text {ex }}=0.25$. A variable number $K$ of $\mathrm{UE} ;$ are placed within the network using exclusion zones of radius $r_{\mathrm{ex}_{\mathrm{mb}}}=0.01$. The outage constraint is set to $\hat{\epsilon}_{\text {channel }}=0.1$ and $\operatorname{SNR}=10 \mathrm{~dB}$.

Fig. 4.2 shows the ASE as a function of the ratio $K / M$, of both ERP and ETP policies in an unshadowed environment as well as in the presence of shadowing. Results are shown for an MCC system using the value of $\left\{r_{\mathrm{ci}}, r_{\max }\right\}$ that maximizes ASE, as well as a conventional system that limits each UE to being served by no more than one $\overline{B S}$, The figure shows the advantage of using $\mathrm{MCC}$ and the superior ASE of the ETP policy. The figure shows that shadowing actually improves the performance, especially when MCC is used. This is because shadowing can sometimes cause the effective distance between a $\mathrm{UE}$ and its serving $\overline{\mathrm{BS}} \mathrm{s}(\mathrm{s})$ to 


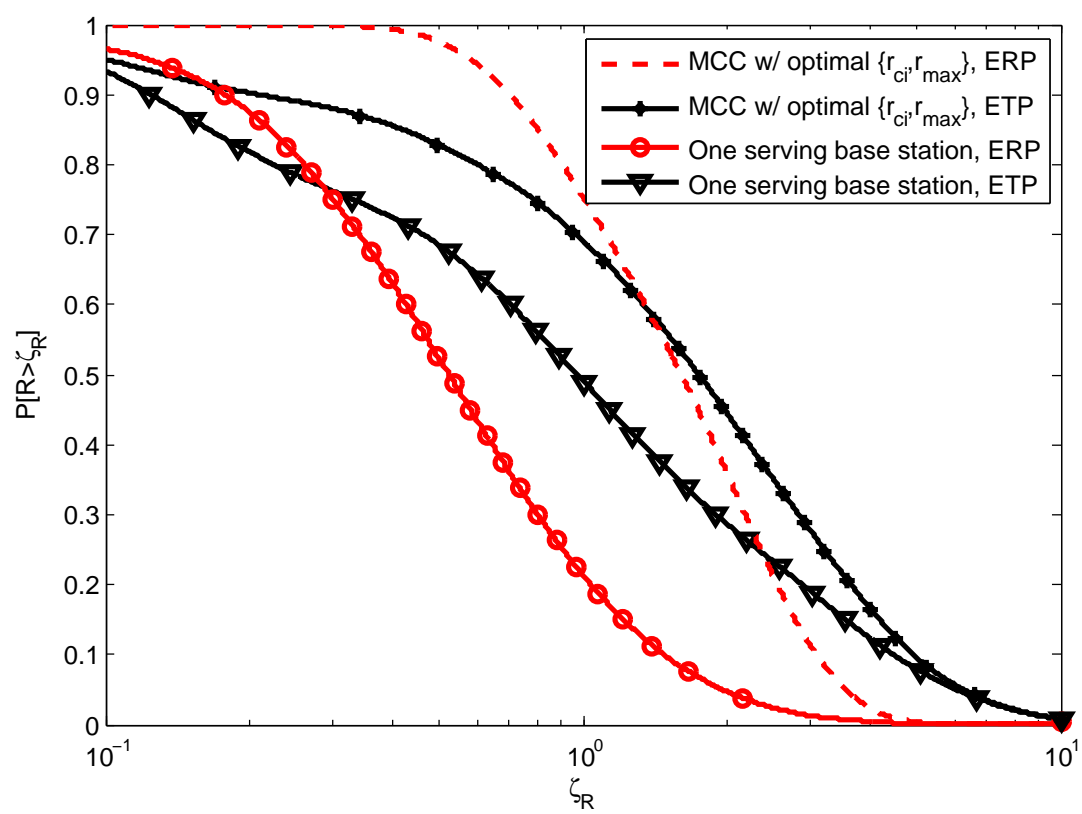

Figure 4.3: $\quad$ CCDF of $R$ for ERP and ETP policy for a half loaded system $(K / M=8)$ in Shadowing $\left(\sigma_{s}=8 \mathrm{~dB}\right)$.

decrease, while its effective distance to the interfering $\overline{\mathrm{BS}}$; is increased. When this situation occurs, the rate can be significantly increased. While shadowing does not induce extremely favorable conditions very often, when it does the improvement in rate is significant enough to cause the average to increase.

The fairness of a particular power allocation policy can be further visualized by plotting the $\overline{\mathrm{CCDF}}$ of $R$, which is the probability that $R$ exceeds a threshold $\zeta_{R}$; i.e., $P\left[R>\zeta_{R}\right]$. Fig. 4.3 shows the CCDF of $R$ in presence of shadowing $\left(\sigma_{s}=8 \mathrm{~dB}\right)$, using the ETP and ERP policies when the system is half loaded $(K / M=8)$. The CCDF curves for the ERP policy are steeper than they are for the ETP policy, indicating less variability in the provided rates. The lower variability in rate corresponds to improved fairness. For instance, all users are provided rates of at least $\zeta_{R}=0.1$ under the ERP policy under $\mathrm{MCC}$. However, when the ETP policy is used, $95 \%$ of the users are provided rates of at least $\zeta_{R}=0.1$. This implies that a significant fraction of the users are provided with lower rates when the ETP policy is used. 


\subsubsection{MBSFN Networks}

MBSFN is a transmission mode defined in the LTE standard [91], and in particular for the multimedia broadcast multicast service (MBMS) [95]. MBSFN is designed to send multicast or broadcast data as a multicell transmission over a synchronized single-frequency network (SFN). A group of those cells that are targeted to receive the same data is called an $M B S F N$ area. An MBSFN area can consist of multiple cells, and a single cell can also be part of multiple, up to eight, MBSFN areas. MBSFN enables the efficient delivery of applications such as mobile TV, radio broadcasting, file delivery and emergency alerts without the need of additional expensive licensed spectrum and without requiring new infrastructure and end-user devices. The transmissions from the different $\overline{\mathrm{BS}} \mathrm{s}$ in an MBSFN area are tightly synchronized so as to avoid [SI and the MBSFN transmission appear to a UE as a transmission from a single large cell over a time-dispersive channel (each BS transmission appears as a separate multipath component). The usage of MBSFN transmission obviously requires not only time synchronization among the cells participating in an MBSFN area, but also usage of the same set of radio resources in each of the cells for a particular service. This coordination is done by the multi-cell/multicast coordination entity (MCE), which is a logical node in the radio-access network handling allocation of radio resources and transmission parameters across the cells in the MBSFN area. MBSFN transmission provides several benefits:

1. Increased received signal strength, especially at the border between cells within the same MBSFN area;

2. Reduced interference, since the signal received from neighboring cells within an MBSFN area appears as a useful information, and not as interference;

3. Additional diversity against fading, since the transmission takes place on separated geographically locations, making the overall channel appear as highly time-dispersive.

Despite the literature on MBSFN networks (i.e, 96 99]), which uses a classical approach to model cellular networks, following from Chap. 3 a more accurate spatial model is used here. In particular, an MBSFN network is synthesized by clustering macrocells formed by 


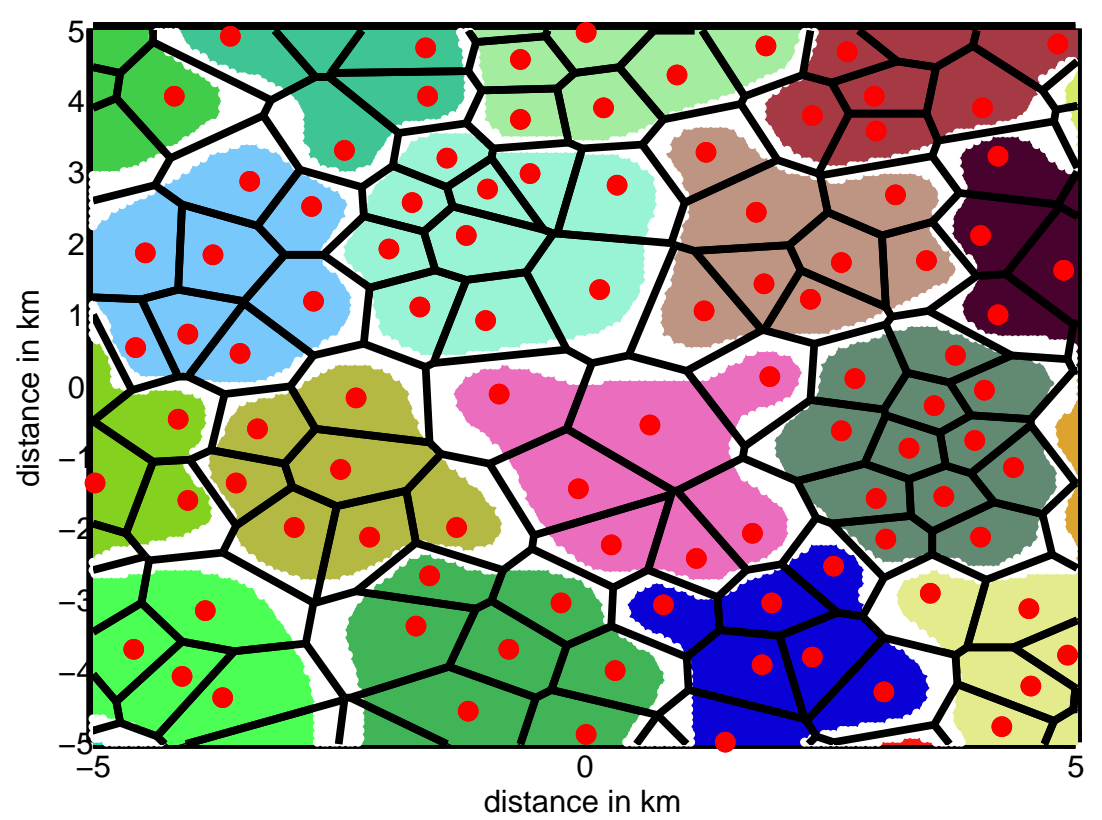

Figure 4.4: Close-up of an example network topology. The BSs locations are given by the large filled circles, the Voronoi tessellation shows the radio cell boundaries, and MBSFN areas are illustrated with different colors. The white areas are the portion of the network for which the outage probability is above a typical value of $\hat{\epsilon}_{\text {channel }}=0.1$.

drawing $\overline{\mathrm{BSS}}$, from a $\mathrm{UCP}$ in order to form the MBSFN areas [100], as shown in Fig. 4.4 . $M$ BSs are deployed according with the constrained spatial model within a square area $|\mathcal{A}|$ with sides of length $d_{\text {net }}$. The network comprises $S_{\text {mbsfn }}$ MBSFN areas, which are formed as follows. Inside the network arena, $\left\{Z_{1}, \ldots, Z_{S_{\mathrm{mbsfn}}}\right\}$ points are picked according to a regular hexagonal grid and equally separated by $d_{\mathrm{sfn}}$. The $z^{\text {th }}$ MBSFN area is then formed by the radio cells of all $\overline{\mathrm{BS}} ;$ that are closer to the location of $Z_{z}$. To facilitate the analysis, the coverage area of the network is discretized into a large number of points, and the variable $Y_{j}$ is used here to indicate the location of the $j^{\text {th }}$ point within the network. Let $\mathcal{G}_{j, z}$ denote the set of the indexes of the $\mathrm{BS}$; that belong to the $z^{\text {th }}$ MBSFN area and serving location $Y_{j}$. Furthermore, let $\mathcal{Z}_{j}$ denote the index of the MBSFN area that covers the location $Y_{j}$ and $\mathcal{N}_{j}=\left|\mathcal{G}_{j, \mathcal{Z}_{j}}\right|$ be the number of $\overline{\mathrm{BS}}$; that belongs to that area.

In a MBSFN OFDMA network, the transmissions are tightly synchronized and it is possible to combine the signals that are sent by all BS $X_{i}, i \in \mathcal{G}_{j, \mathcal{Z}_{j}}$ and received at the UE, located at position $Y_{j}$, if the signals arrive within the extended cyclic prefix, which is fixed to be equal to $T_{E C P}=16.7 \mu s[101]$. The signal from $\mathrm{BS} X_{i}, i \in \mathcal{G}_{j, \mathcal{Z}_{j}}$ to the UE at location 
$Y_{j}$ is then included in the maximal-ratio combining (MRC) combined signal passed to the demodulator, if $\left\|X_{i}-Y_{j}\right\|<d_{\max }$, where $d_{\max }=c / T_{E C P}=5 \mathrm{Km}$, where $c=3 \cdot 10^{8} \mathrm{~m} / \mathrm{s}$ and corresponds to the speed of light, otherwise it results in ISI. The set $\mathcal{G}_{j, \mathcal{Z}_{j}}$ contains the indices of those $\mathrm{BS}$ s that are closer to $Z_{\mathcal{Z}_{j}}$ and they are located such that their signals arrive at $Y_{j}$ within the extended cyclic prefix. The set $\mathcal{G}_{j, \mathcal{Z}_{j}}$ is then selected such that $i \in \mathcal{G}_{j, \mathcal{Z}_{j}}$ if $\left\|Z_{\mathcal{Z}_{j}}-X_{i}\right\|<\left\|Z_{s}-X_{i}\right\|, \forall s \neq \mathcal{Z}_{j}$ and also if $\left\|X_{i}-Y_{j}\right\|<d_{\max }$.

Assume block fading for the duration of a MBSFN subframe and log-normal shadowing, which is characterized by the normalized autocorrelation function $\mathcal{R}(\Delta x)$, where $\Delta x$ is the change in distance. The normalized autocorrelation function can be described with sufficient accuracy by an exponential function as 102

$$
\mathcal{R}(\Delta x)=\exp \left\{-\frac{\|\Delta x\|}{d_{\text {corr }}} \ln 2\right\}
$$

with the decorrelation length $d_{\text {corr }}$, which is dependent on the environment. For the vehicular test environment (VTE), [103] proposes $d_{\text {corr }}=20 \mathrm{~m}$. This correlation works satisfactorily for distances up to approximately $500 \mathrm{~m}$. Furthermore, the distance-dependent fading model given by (3.7) is used, with $r_{\text {los }}=r_{\text {exbs }}$.

In this case the normalized received power at the location $Y_{j}$, when the $\mathrm{BS} X_{i}$ transmits, is

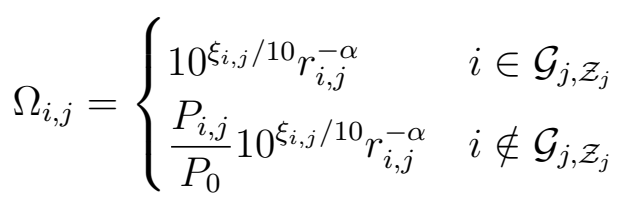

and the conditioned outage probability can be evaluated by substituting (4.16) in 4.9).

\section{Performance Analysis}

In order to analyze the network performance, the area below an outage threshold (ABOT) is used, which is defined as the fraction of the network realization $t$ that provides an outage probability (averaged over the fading) that meets a threshold

$$
\mathcal{A}_{\text {bot }}^{(t)}=P\left[\epsilon_{j}<\hat{\epsilon}_{\text {channel }}\right]
$$

The ABOT is an indicator of the percentage of the network that will successfully receive the broadcast. In Fig. 4.4 it is shown a portion of an example network, where the area in 
white is the portion of the network for which the outage probability is above $\hat{\epsilon}_{\text {channel }}=0.1$ with $\beta=0 \mathrm{~dB}, \mathrm{SNR}=10 \mathrm{~dB}$ and $\alpha=3.5$, which as expected corresponds to the edge of the MBSFN areas, that are here illustrated with different colors and obtained by fixing $d_{\text {sfn }}=3$. The $\mathrm{BS}$ exclusion radius is fixed to $r_{\text {ex } \mathrm{bs}}=0.5, M=400 \underline{\mathrm{BS}}$ s are deployed into a square network arena with side of length $d_{\text {net }}=20 \mathrm{Km}$. The BS locations are given by the large filled circles and the Voronoi tessellation shows the radio cell boundaries that occur in the absence of shadowing.

After computing $\mathcal{A}_{\text {bot }}^{(t)}$ for $\Upsilon$ network topologies, its spatial average can be computed as following

$$
\overline{\mathcal{A}}_{\mathrm{bot}}=\frac{1}{\Upsilon} \sum_{t=1}^{\Upsilon} \mathcal{A}_{\mathrm{bot}}^{(t)}
$$

where in the following $\Upsilon=1,000$.

In order to determine the performance of a typical network the following approach is used here. Draw a realization of the network by placing $\mathrm{BS}$ s according to a uniform clustering model with density $\lambda=M /|\mathcal{A}|$. Group the BS; into MBSFN areas. Compute the path loss from each BS to a very large number of locations, selected such that they form an extremely dense grid that covers the entire network arena, applying randomly generated correlated shadowing factors if shadowing is present. Determine the outage probabilities by using (4.9) parameterized by the SINR threshold for all locations. For each of the previously selected locations within the network, determine whether or not it satisfies the outage constraint. Notice, that in order to exclude edge effects, the performance is evaluated only in the center $10 \times 10 \mathrm{Km}^{2}$ portion of the network. As in the previous subsection, the SINR threshold is related to the code rate $R$ through the Shannon capacity for complex discrete-time AWGN channels.

Fig. 4.5 shows the $\mathrm{ABOT}$ as function of the outage threshold parameterized for three values of $d_{\text {sfn }}$ when $\lambda=0.5$ and $R=0.5$. Fig. 4.5 shows that an increase in the size of the MBSFN areas results in an improvement in performance, but only until a certain value. After a certain value of $d_{\text {sfn }}$, the [SI starts to increase and the regions at the edge of the MBSFN areas don't get any more benefit by increasing them furthermore. Fig. 4.6 shows the area below an outage threshold as function of the minimum separation among $\overline{\mathrm{BS}}$; when 


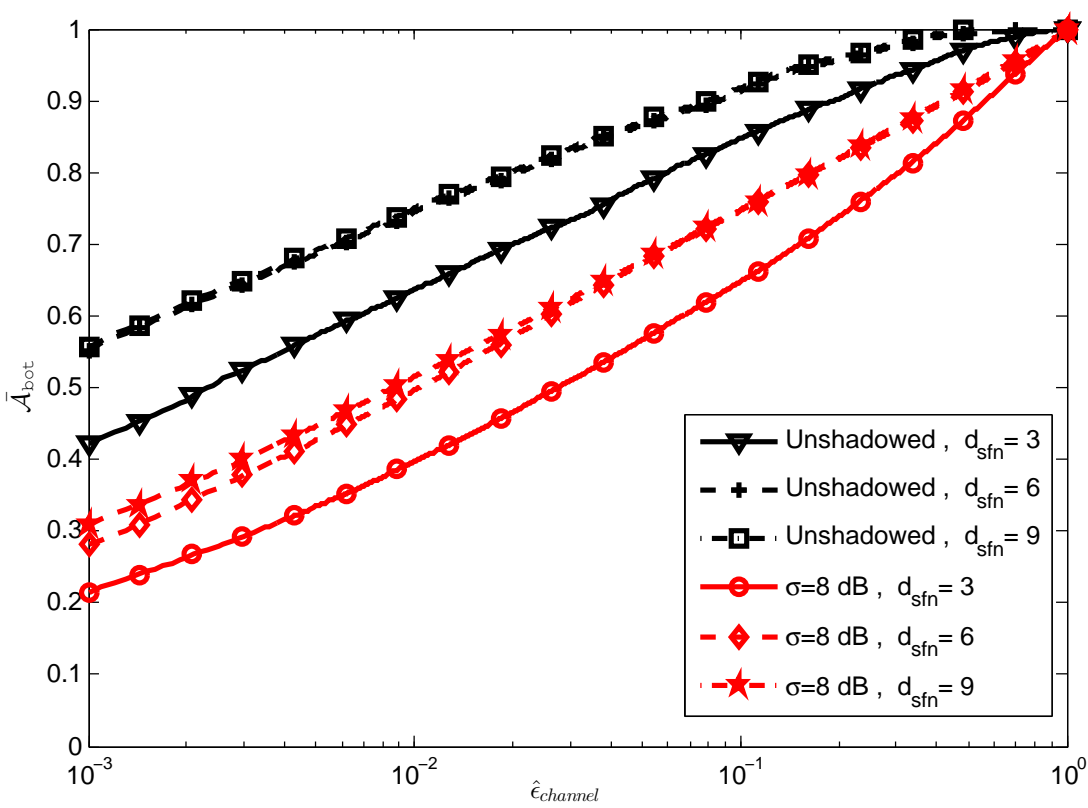

Figure 4.5: ABOT as a function of the threshold $\hat{\epsilon}_{\text {channel }}$ for both a shadowed $\left(\sigma_{s}=8 \mathrm{~dB}\right)$ and unshadowed environment with $R=0.5$.

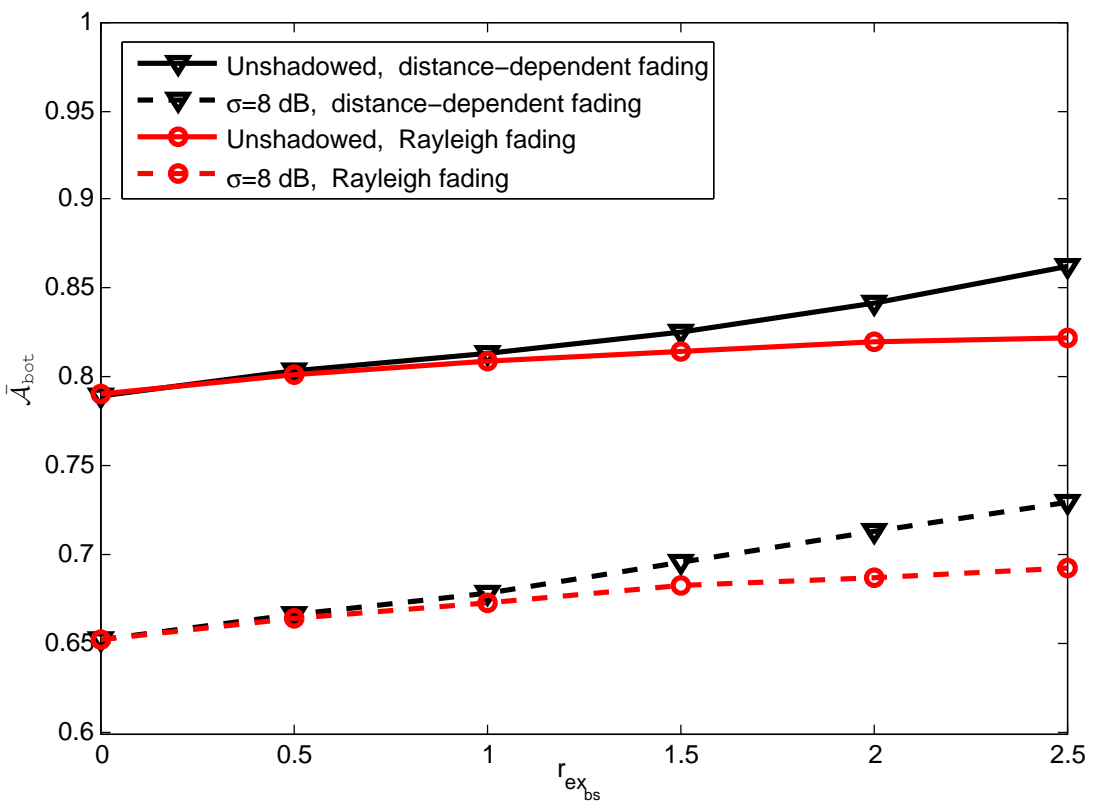

Figure 4.6: ABOT as a function of the minimum separation among BSs when $R=0.1$, $\lambda=0.1$ and $d_{\mathrm{sfn}}=6$. 
$R=0.1, \lambda=0.1$ and $d_{\mathrm{sfn}}=6$. The curves are shown for both a shadowed $\left(\sigma_{s}=8 \mathrm{~dB}\right)$ and an unshadowed scenario and for both Rayleigh fading and a distance-depending fading. Fig. 4.6 shows that shadowing is always highly detrimental, the fraction of network that succeeds to meet an outage constraint increases when BSs have a higher minimum separation, and this effect is more prominent under a distance-depending fading.

\subsection{Chapter Summary}

This chapter presented a new approach for modeling and analyzing the performance of cooperative networks that employ diversity combining. The chapter illustrates the performance benefits that arise due to spatial diversity. The analysis is characterized by a new closed-form expression for the conditional outage probability, when signals arriving over different paths are combined at the receiver in the presence of noise and interference, where the conditioning is with respect to the network geometry. The channel model accounts for path loss, shadowing, and Nakagami fading, and the Nakagami fading parameters do not need to be identical for all links.

This chapter continues by applying this accurate analysis to two cases of study. First, it models and analyzes the performance of a DS-CDMA MCC downlink and provides insight regarding how the cell edge should be defined. The results show that, as expected, there is an improvement in performance when MCC is used, rather than a conventional cellular network, but the network needs a proper optimization. Finally, this chapter focuses on accurately and efficiently modeling an MBSFN network, by combining the new closed form expression for the outage probability, derived at the beginning of this chapter, and a constrained random spatial model, which is used to synthesize more realistically such a network. The results show that an increase in the size of an MBSFN areas leads to an improvement in performance only until a certain dimension (and number of cooperative $\mathrm{BS}$ ) is reached, since the ISI starts to become more prevalent and their effect more detrimental. 


\section{Chapter 5}

\section{The Role of Complexity in Cellular Networks}

Compared with today's networks, future mobile networks will need to cope with a dramatic increase in the density and demand of users, and they will be characterized by tighter hard real-time timing and protocol constraints. These constraints are described by mobile communications standards such as 3GPP LTE [101] and must not be violated. In order to fulfill these constraints, a pre-defined amount of computational resources must be provided such that downlink and uplink processing can be performed within a given time interval, and this must be done in an efficient and very cost effective way.

Recently the concept of Cloud-RAN has been introduced [30], which enables computational load balancing across multiple $\mathrm{BS}$ s to avoid peak-provisioning. For this novel architecture, one of the goals is to dimension the computational assets of the network to exploit the computational diversity present in a pool of resources, while still guaranteeing the communication service requirements of each individual cell. The utilization of processing resources should be maximized for a given service guarantee. These goals require a framework that enables the prediction of the required data processing resources under a given performance constraint.

This chapter, after an introductory section on the Cloud- $[\mathrm{RAN}$ architecture, considers its computational requirements with the goal of illuminating the benefits of pooling computational resources. A new analytical framework is introduced to model, analyze the perfor- 
mance, and determine the data processing requirements. The model is used to quantify the computational load associated with the centralized processing of uplink signals in the presence of block Rayleigh fading, distance-dependent path loss, and fractional power control. Several new performance metrics are defined, including computational outage probability, outage complexity, computational gain, computational diversity, and the complexity-rate tradeoff. The validity of the analytical framework is confirmed by comparing it numerically with a simulator compliant with the 3GPP LTE standard. Using the developed metrics, it is shown that centralizing the computing resources provides a higher net throughput per computational resource as compared to local processing.

\subsection{Cloud-RAN: A Brief Introduction}

Traditional cellular network are composed of stand-alone $\overline{\mathrm{BS}}$ s, which perform both radio and baseband processing functionality locally at or near the cell site. In this architecture, most of the hardware is placed in a base station cabinet located right below the antenna. Starting from the third generation cellular networks $(3 \mathrm{G})$, the two groups of functionalities have been physically separated. The radio functionalities are performed by a remote radio head ( $(\mathrm{RRH})$, which is installed on top of the tower, close to the antenna, in order to reduce the cable loss. The baseband functionalities are performed at a baseband unit ( $\overline{\mathrm{BBU}})$ or data unit (DU), which can be placed further away from the $\overline{R R H}^{1}$, and it is connected to it through optical fiber or microwave connection. This architecture has the advantage that the $\mathrm{BBU}$ can be conveniently placed enabling a faster and less expensive maintenance and upgrade of the system. However, due to the enormous increase in the number of users and data shared, the current cellular architecture is reaching the limit of sustaining the amount of data traffic on the network. Especially in densely populated areas, it is no longer acceptable and sustainable to only increase the number of $\overline{\mathrm{BS}}$ s in order to provide smooth network accesses to users. The two main reasons are: i) economical since nowadays the deployment

\footnotetext{
${ }^{1}$ Note that the maximum distance at which the BBU can be placed depends on the latency of the medium through which the signal is propagated. For instance for optical fiber, a reasonable maximum distance is $\approx 40 \mathrm{Km}$ when a repeater is not required. In this case, the medium produces a propagation delay of about $0.1 \mathrm{~ms}$.
} 


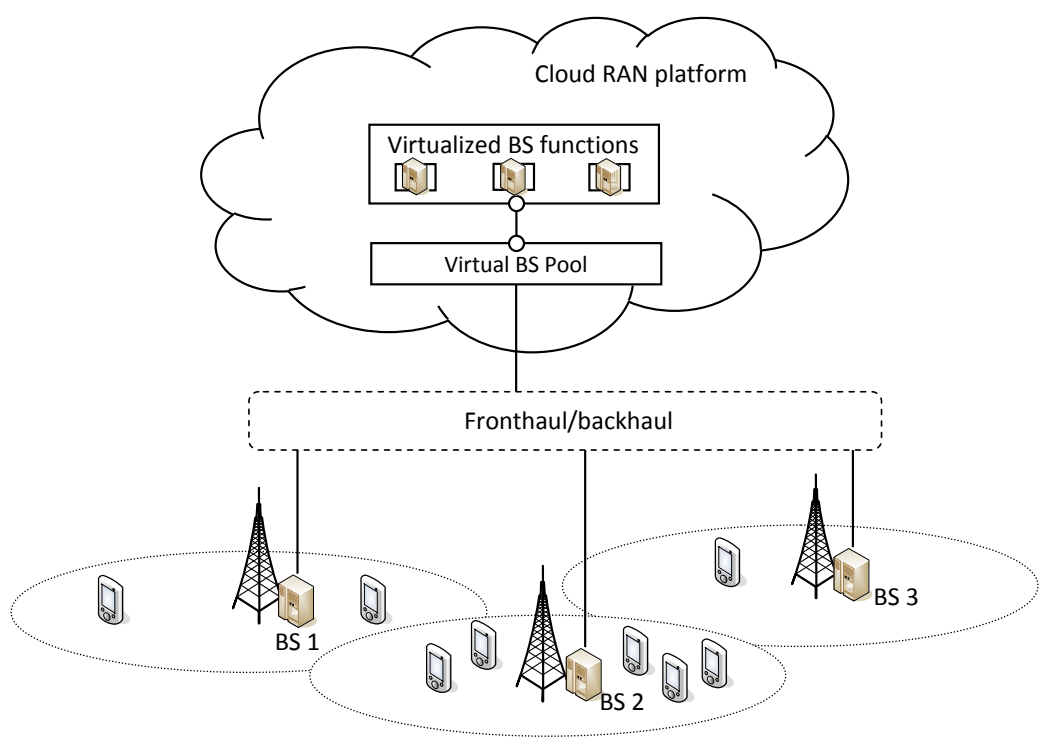

Figure 5.1: Illustration of the Cloud-RAN architecture.

of a new $\mathrm{BS}$ is very cost effective; and ii) due to the increment of the interference among $\mathrm{BS}$, since the likelihood that the same radio resources are used increases.

In 2010 a new architecture has been proposed [30, 32, 104], which have been named CRAN, where the letter $\mathrm{C}$ can be interpreted as: Cloud, Centralized, Cooperative radio, Collaborative or Clean ${ }^{2}$. The Cloud- $\underline{\mathrm{RAN}}$ is based on two tenets: centralization and virtualization of BS baseband processing. In a Cloud-RAN network, the radio protocol stack is executed by different physical entities. For a given group of cells, the lower parts of the protocol stack are executed at the $\underline{\mathrm{RRH}}$; , while the upper parts are executed at a central entity, called the Cloud-RAN platform. The $\overline{\mathrm{RRH}}$; use dedicated hardware, while the CloudRAN is typically implemented with commodity general-purpose hardware. A Cloud-RAN enables advanced interference coordination strategies while leveraging the elastic provisioning of resources provided by the cloud. It is particularly well suited for dense deployments, such as within a large building where the $\mathrm{RRH}$; are connected via fiber and many cells are underutilized. The Cloud-RAN concept is illustrated Fig. 5.1. A Cloud- $\mathrm{RAN}$ system consists of multiple $\mathrm{RRH}$, which perform only simple processing of the input signal, including

\footnotetext{
${ }^{2}$ The $\mathrm{C}$ is often interpreted as Centralized when the aim is to describe a system where the baseband processing for a group of cells is done at a central processing system. It is interpreted as Cloud when the processing is done on a pool of computing assets, made possible trough the use of virtualization. Note that in the following the two terms are interchangeable.
} 
sampling and optionally performing a fast Fourier transform (FFT) operation, a fronthaul or backhaul connection, and a central data center. The data center manages a virtual BS pool composed of a scalable number of virtual machines $(\underline{\mathrm{VM}} \mathrm{s})$, each representing one or more $\overline{\mathrm{BS}} \leqslant$ and performing the majority of the baseband processing. Based on the actual traffic and resulting computational demand, each VM is provisioned with resources that can be elastically adjusted.

The major benefits of Cloud-[RAN have been unveiled in [30]. Since a Cloud-[RAN enables computational load balancing across multiple $\overline{\mathrm{BS}}$; , it allows a more efficient use of $\mathrm{BBU}$ and reduces the cost of $\overline{\mathrm{BSS}}$ s deployment and operation. The required baseband processing capacity of the pool is expected to be smaller than the sum of capacities of single $\mathrm{BS} s$. In particular, Bhaumik et al. have predicted in 105 that Cloud-RAN can save up to $22 \%$ of the data processing resources, by using the OpenAir [106] LTE implementation and realworld measurements. Cloud-RAN is also expected to lead to save power consumption by about $67 \%-80 \%$ compared to traditional RAN architecture. Due to the centralized nature of this architecture, it provides increased flexibility in network upgrades and adaptability to non-uniform traffic, which is particular useful in areas where the traffic demand changes abruptly during the day. Furthermore, advanced features of LTE-A, such as coordinated multi-point (CoMP) and interference mitigation, can be efficiently supported by the CloudRAN, which is essential especially for small-cell deployments.

A Cloud- $\mathrm{RAN}$ architecture, which is characterized as mentioned above by several remarkable benefits, has although several technical challenges that need to be addressed. One of the main limitations of this architecture is the backhaul, which needs to be sufficiently fast and with low latency, in order to meet very hard real-time timing. Furthermore, the computational load required to perform uplink or dowlink processing cannot exceed the available resources, otherwise the signal cannot be properly decoded in time. This last limitation is strictly dependent on the computational complexity of the decoder, and its role and effect is subject of study of this chapter. 


\subsection{Complexity model}

Consider a cloud group containing $N_{\text {cloud }}$ BSs whose signals are jointly processed in a virtual $\mathrm{BS}$ pool. Let $\bar{\gamma}=\mathbb{E}[\gamma]$ indicate the average SINR, In the following, it is assumed that the channel gain remains fixed for the duration of one transport block (TB), but varies independently from $\mathrm{TB}$ to TB, which corresponds to a block-fading model. For each $\mathrm{TB}$, the channel is conditionally subject to AWGN Under the assumption of infinite block-length, the capacity for a given instantaneous SINR is given by $\log _{2}(1+\gamma)$, which provides an upper bound on rate. In practice, the rate is selected for a given $\gamma$ from a set of modulation and coding schemes (MCS $)$ in such a way that a constraint on outage probability is satisfied. Because no finite-length code satisfies the capacity limit with equality, the rate is selected to be below the corresponding capacity limit according to some margin trough link adaptation.

\subsubsection{Link Adaptation}

LTE features adaptive modulation and coding using turbo codes and hybrid automatic repeat request (HARQ). The BS (called an eNodeB) commands the UE to transmit using one of $N_{R}=27$ different $\mathrm{MCS}$, with $R_{k}$ denoting the rate of $k^{\text {th }} \mathrm{MCS}, k \in\left\{1, \ldots, N_{R}\right\}$. Each MCS is identified by an MCS index, $I_{\mathrm{mcs}}=\{0, \ldots, 26\}$, and is characterized by a different combination of code rate and modulation format [107, 108]. Three kinds of modulation are used: QPSK $\left(0 \leq I_{\mathrm{mcs}} \leq 10\right)$, 16-QAM $\left(11 \leq I_{\mathrm{mcs}} \leq 20\right)$, and 64-QAM $\left(21 \leq I_{\mathrm{mcs}} \leq 26\right)$. When a TB is larger than 6144 bits, it is segmented into $C_{k}$ code blocks ( $\mathrm{CB}$ ), each of which conveys $D_{k}$ information bits. As advocated later, the selection of the MCS should take into account the complexity of the turbo decoder, and in particular, the number of expected iterations required to meet the outage constraint is the key factor in making the selection. Suppose that the turbo decoder is run with a certain number of maximum iterations $L_{\max }$. Let $\gamma_{k}^{R}$ indicate the minimum SINR for which the $k^{\text {th }}$ MCS satisfies the outage constraint after the $L_{\text {max }}$-th iteration, on average. The value of $\gamma_{k}^{R}$ for each MCS can be obtained as follows. Simulations are used to obtain code block error rate (CBLER) curves for each possible MCS and for an arbitrarily large number of maximum iterations. For the $k^{\text {th }}$ MCS, $\gamma_{k}^{R}$ is selected to be the value of SNR for which the transport block error rate (TBLER) 
satisfies a particular constraint for the channel outage $\hat{\epsilon}_{\text {channel }}$ (a transport block error occurs when any of the code blocks in a transport block fails).

It is well known that the complexity of a turbo decoder depends on how much the channel SINR exceeds the capacity limit. If a turbo code of rate $R_{k}$ is transmitted over a channel whose capacity is sufficiently higher than $R_{k}$, then typically only a few iterations are required. Thus, to manage complexity, an SINR margin $\Delta \gamma$ may be used. With such a margin, the SINR must be at least $\Delta \gamma$ above $\gamma_{k}^{R}$ in order to select the $k^{\text {th }}$ MCS, The rate selection for channel SINR $\gamma$ and SINR margin $\Delta \gamma$ can be represented by the function

$$
\mathcal{R}(\gamma, \Delta \gamma)= \begin{cases}R_{1} & \text { if } \frac{\gamma}{\Delta \gamma} \leq \gamma_{1}^{R} \\ R_{k} & \text { if } \gamma_{k}^{R}<\frac{\gamma}{\Delta \gamma} \leq \gamma_{k+1}^{R} \\ R_{N_{R}} & \text { if } \gamma_{N_{R}}^{R}<\frac{\gamma}{\Delta \gamma}\end{cases}
$$

\subsubsection{Complexity Model}

The computational effort required to decode a turbo code is linear in the number of iterations and in the number of (information) bits. Thus a reasonable metric for computational effort is the bit-iteration. For a given SINR $\gamma$, let the random variable $L_{r}(\gamma, \Delta \gamma)$ be the number of iterations required to decode a particular $\mathbb{C B}$, and $\mathbb{E}\left[L_{r}(\gamma, \Delta \gamma)\right]$ be the average number of iterations required to decode a $\mathrm{CB}$. The expected decoding complexity (averaged over the number of iterations required per $[\mathrm{CB}$ ) expressed in bit-iterations per channel use (pcu) can be evaluated as

$$
\mathcal{C}(\gamma, \Delta \gamma)=\frac{D_{k} C_{k} \mathbb{E}\left[L_{r}(\gamma, \Delta \gamma)\right]}{S_{\mathrm{re}}}
$$

where $S_{\mathrm{re}}$ is the number of channel uses or resource elements $(\mathrm{RE} s)$ required to convey the TB. The expected number of decoding iterations can be found using the same set of simulation curves used to determine the MCS thresholds $\gamma_{k}^{R}$. For a given rate $\mathcal{R}(\gamma, \Delta \gamma)$, the simulation results will show the CBLER for each iteration up to the $L_{\text {max }}$-th iteration. These CBLER curves can be interpreted as the PMF of the number of iterations at $\gamma$, and from the PMF coefficients, the average number of iterations is easily computed.

To enable a deeper analysis of complexity issues, it is essential to build an accurate yet wieldy complexity model. The groundwork for such a model is provided by [109], which 
accurately predicts the power consumption of a decoder for a given code rate, coding scheme, channel, and decoder type. According to [109, Eq. (4) and (9)], the expected decoding complexity under a given instantaneous SINR $\gamma$ and SINR margin $\Delta \gamma$ can be modeled as

$$
\begin{aligned}
\mathcal{C}(\gamma, \Delta \gamma) & =\frac{\mathcal{R}(\gamma, \Delta \gamma)}{\log _{2}(\zeta-1)} \log _{2}\left[\frac{-\log _{10}\left(\hat{\epsilon}_{\text {channel }}\right)}{K^{\prime}\left[\log _{2}(1+\gamma)-\mathcal{R}(\gamma, \Delta \gamma)\right]^{2}} \frac{\zeta-2}{\zeta}+\frac{2}{\zeta}\right] \\
& \approx \frac{\mathcal{R}(\gamma, \Delta \gamma)}{\log _{2}(\zeta-1)} \log _{2}\left[\frac{-\log _{10}\left(\hat{\epsilon}_{\text {channel }}\right)}{K^{\prime}\left[\log _{2}(1+\gamma)-\mathcal{R}(\gamma, \Delta \gamma)\right]^{2}} \frac{\zeta-2}{\zeta}\right] \\
& =\frac{\mathcal{R}(\gamma, \Delta \gamma)}{\log _{2}(\zeta-1)}\left[\log _{2}\left(\frac{\zeta-2}{K\left(\hat{\epsilon}_{\text {channel }}\right) \zeta}\right)-2 \log _{2}\left[\log _{2}(1+\gamma)-\mathcal{R}(\gamma, \Delta \gamma)\right]\right]
\end{aligned}
$$

where $\zeta$ is a parameter of the model related to the connectivity of the decoder when represented as a graph,

$$
K\left(\hat{\epsilon}_{\text {channel }}\right)=-\frac{K^{\prime}}{\log _{10}\left(\hat{\epsilon}_{\text {channel }}\right)},
$$

$K^{\prime}$ is a parameter of the model, and $\hat{\epsilon}_{\text {channel }}$ is a constraint on the channel outage probability. For the sake of simplicity $K\left(\hat{\epsilon}_{\text {channel }}\right)$ is denoted by $K$ in the remainder of this dissertation.

When evaluating (5.3), the function $\mathcal{R}(\gamma, \Delta \gamma)$ is defined by (5.1). The relationship between each threshold $\gamma_{k}^{R}$ and the corresponding rate $R_{k}$ in 5.1 can be modeled as

$$
R_{k}=\log _{2}\left(1+\frac{\gamma_{k}^{R}}{\nu}\right)
$$

where $\nu$ is a parameter that models the gap between the capacity at $\gamma_{k}^{R}$ and the SINR for the actual code to meet the performance objective at rate $R_{k}$. The values of $\left(\zeta, K^{\prime}, \nu\right)$ could be found for each MCS by statistically fitting the empirically observed complexity to the complexity predicted by the model. However, it is more convenient to have values for these parameters that are common to all the MCS5.

\section{Example}

As an example, let consider an LTE system that uses normal cyclic prefix and a $10 \mathrm{MHz}$ bandwidth corresponding to 50 resource blocks $(\underline{\mathrm{RB}})$. However, since up to $5 \underline{\mathrm{RB}}$ s must be reserved for the physical uplink control channel and the number of $\mathrm{RB}$; must be a multiple of 2, 3, or 5 [107, 108, the transmitted signal occupies 45 RB;. Since there are 12 informationbearing single-carrier frequency division multiple access (SC-FDMA symbols per sub-frame 


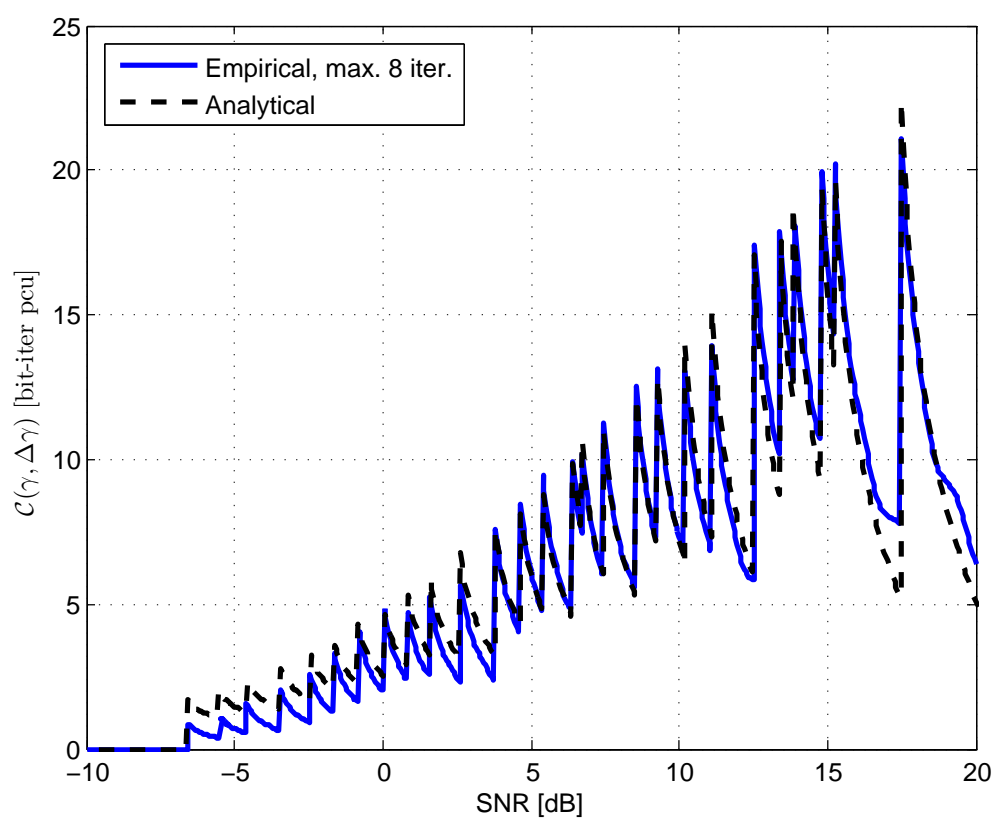

Figure 5.2: Complexity as a function of the SNR obtained both through simulations (blue) and based on (5.3) (black curve), when the maximum number of iterations used to decode a $\mathrm{CB}$ is limited to eight.

and 12 sub-carriers per $\mathrm{RB}$, it follows that $S_{\text {re }}=45 \times 12 \times 12=6480$. The MCS selection is implemented according to (5.1) with threshold found for $L_{\max }=8$ iterations, an outage constraint $\hat{\epsilon}_{\text {channel }}=0.1$, and no excess margin $(\Delta \gamma=0 \mathrm{~dB})$.

For this settings, the complexity computed empirically using $(5.2)$ is shown in Fig. 5.2 by the blue curve, while the black curve shows the complexity predicted by the complexity model described above. For the complexity model, the values of $\left(\zeta, K^{\prime}, \nu\right)$ have been found in order to have the best fit with the empirical complexity, and they are found to be: $\zeta=6, K^{\prime}=0.2$, and $\nu=0.2 \mathrm{~dB}$. As can be seen, these two curves are quite similar ${ }^{3}$, demonstrating the effectiveness of the model. Fig. 5.2 further shows a highly volatile behavior, which results from the link-adaptation process. When the system operates at the same MCS (rate), a decoder requires fewer iterations as the SNR increases. However when link adaptation is performed, the system requires more complexity each time it moves towards a higher MCS, since it operates closer to capacity, which is the cause of the peaky behavior in Fig. 5.2 .

\footnotetext{
${ }^{3}$ Note that a more accurate match could be achieved by determining a different set of $\left(\zeta, K^{\prime}, \nu\right)$ for each MCS, but this significantly complicates the notation.
} 


\subsection{Performance Metrics}

This section provides the definition for several performance metrics, which are used throughout this chapter to evaluate the performance of a Cloud-RAN system and highlight its benefits over a distributed implementation.

\subsubsection{Computational outage probability}

Fig. 5.2 shows that the required computational complexity to decode a TB may differ significantly depending on the SINR, Furthermore, when the system is distributed, each BS will be provisioned with a limited amount of computational resources $\mathcal{C}_{\max }$, while in a centralized system the virtual BS pool will be equipped with a total limited amount of computational resources, which scales linearly with the number of $\overline{\mathrm{BS}}$;. If the total required computational effort exceeds the computational resources before a decoding deadline is reached, a computational outage will occur. From the operator's perspective, a computational outage is no different than a channel outage, because in both cases the information is not made available to the end user.

More formally, the computational outage probability is defined by [110]

$$
\epsilon_{\text {comp }}\left(N_{\text {cloud }}\right)=\mathbb{P}\left[\sum_{i=1}^{N_{\text {cloud }}} \mathcal{C}\left(\gamma_{i}, \Delta \gamma\right)>N_{\text {cloud }} \mathcal{C}_{\max }\right]
$$

When a computational outage occurs, the centralized processor is unable to finish the decoding of at least one uplink user. However, when this condition occurs it is still possible that some of the uplink transport blocks can be decoded, even though at least one of the blocks has failed.

\subsubsection{Outage complexity}

The outage complexity $\mathcal{C}_{\text {outage }}\left(\hat{\epsilon}_{\text {comp }}, N_{\text {cloud }}\right)$ is the minimum amount of computational resources required to satisfy an outage constraint on the per-cell computational outage probability $\hat{\epsilon}_{\text {comp }}\left(N_{\text {cloud }}\right)$. More formally, the outage complexity is defined as

$$
\mathcal{C}_{\text {outage }}\left(\hat{\epsilon}, N_{\text {cloud }}\right)=\arg \min _{\mathcal{C}_{\max }}\left\{\mathbb{P}\left[\sum_{i=1}^{N_{\text {cloud }}} \mathcal{C}\left(\gamma_{i}, \Delta \gamma\right)>N_{\text {cloud }} \mathcal{C}_{\text {max }}\right] \leq \hat{\epsilon}_{\text {comp }}\left(N_{\text {cloud }}\right)\right\} .
$$


The constraint of the computational outage probability of the system $\hat{\epsilon}_{\text {comp }}\left(N_{\text {cloud }}\right)$ is normalized to a per-cell basis. Assuming independent processing with a non-priority-based scheduler, the constraint for a centralized system is related to the constraint for a distributed system with value $\hat{\epsilon}_{\text {comp }}(1)=\hat{\epsilon}_{\text {comp }}$ as follows

$$
\hat{\epsilon}_{\text {comp }}\left(N_{\text {cloud }}\right)=1-\left(1-\hat{\epsilon}_{\text {comp }}\right)^{N_{\text {cloud }}} .
$$

\subsubsection{Computational gain and diversity}

Using the previous definition of computational outage, the centralization gain of a cloudbased mobile network is determined. In particular, the impact on the performance by centralizing communication-processing resources can be measured in two ways: 1) by considering the reduction of the required computational resources; 2) by examining the improved outage behavior.

The computational gain quantifies the reduction of computational resources made possible by the centralization of processing. When $N_{\text {cloud }} \overline{\mathrm{BS}} \leqslant$ are jointly processed, the computational gain is

$$
g_{\text {comp }}\left(N_{\text {cloud }}\right)=\frac{N_{\text {cloud }} \mathcal{C}_{\text {outage }}\left(\hat{\epsilon}_{\text {comp }}, 1\right)}{\mathcal{C}_{\text {outage }}\left(\hat{\epsilon}_{\text {comp }}, N_{\text {cloud }}\right)}
$$

The asymptotic computation gain is found by letting the number of $\overline{\mathrm{BS}}$; go to infinity,

$$
g_{\text {comp }}^{\infty}=\lim _{N_{\text {cloud }} \rightarrow \infty} g_{\text {comp }}\left(N_{\text {cloud }}\right) .
$$

An alternative metric is the computational diversity, which quantifies the rate of computational improvement as more $\overline{\mathrm{BS}}$ s are jointly processed. This is found by determining the rate at which the computational outage probability is improved as a function of $N_{\text {cloud }}$. In particular, the computational diversity is

$$
d_{\text {comp }}\left(N_{\text {cloud }}\right)=-\frac{\partial \log _{10} \epsilon_{\text {comp }}\left(N_{\text {cloud }}\right)}{\partial N_{\text {cloud }}} .
$$

This is the downward slope of the curve that relates computational outage probability to the number of jointly processed $\mathrm{BS}$. The larger this negative slope, the faster the maximum computational gain (5.10) is achieved. In a mobile network, the computational diversity 
provides an indication for the required number of centralized BSs per data-center. When more $\mathrm{BS} ;$ must be centralized, the backhaul requirements will increase accordingly. Since the slope of the computational diversity has its maximum magnitude when $N_{\text {cloud }}=1$, in the following the derivative of (5.11) is evaluated at $N_{\text {cloud }}=1$ and it is indicated by $d_{\text {comp }}$, dropping the dependency on $N_{\text {cloud }}$.

\subsubsection{Complexity-Rate Tradeoff}

Focusing only on outage complexity ignores the influence of rate. An alternative metric should consider the relationship between the rate and the SINR margin $\Delta \gamma$. As the margin $\Delta \gamma$ increases, the complexity decreases, but so does the rate since the system operates farther away from capacity. Therefore, the complexity-rate tradeoff (CRT) can be quantified, and it measures how much additional complexity is required in order to further improve the rate. In particular, the complexity-rate tradeoff can be evaluated as

$$
t_{\text {comp }}\left(N_{\text {cloud }}\right)=\lim _{\Delta \gamma \rightarrow 0} \frac{\partial r(\gamma, \Delta \gamma)}{\partial \Delta \gamma}\left[\frac{\partial \mathcal{C}_{\text {outage }}\left(\hat{\epsilon}_{\text {cloud }}, N_{\text {cloud }}\right)}{\partial \Delta \gamma}\right]^{-1}
$$

\subsubsection{Average Achievable Rate}

Given a fixed average SINR $\bar{\gamma}$, and a fixed SINR margin $\Delta \gamma$, the rate associated to a given user depends upon the MCS selected based on the quality of channel. Due to the random effect of fading, the code rate selected per user is aleatory. One of the conventional ways to measure system performance involves evaluating the average code rate. Given the distribution of the instantaneous $\operatorname{SINR} \gamma$, the expected code rate can be computed as follows

$$
\begin{aligned}
\mathbb{E}\{R\} & =\sum_{k=1}^{N_{R}} \mathbb{P}\left\{\gamma_{k}^{R} \leq \gamma<\gamma_{k+1}^{R}\right\} \mathcal{R}\left(\gamma_{k}^{R}, \Delta \gamma\right) \\
& =\sum_{k=1}^{N_{R}}\left[F_{\gamma}\left(\gamma_{k+1}^{R}\right)-F_{\gamma}\left(\gamma_{k}^{R}\right)\right] \mathcal{R}\left(\gamma_{k}^{R}, \Delta \gamma\right)
\end{aligned}
$$

where $F_{\gamma}(\gamma)$ is the $\mathrm{CDF}$ of the instantaneous $\mathrm{SINR} \gamma$. 


\subsection{A Framework for Complexity Analysis}

In order to evaluate the previously introduced performance metrics, an analytical framework is developed [111 in this section to quantify the expected outage complexity in a centralized network. In the following, the analysis is first built under the assumption of a fixed average SINR $\bar{\gamma}$, which is true when the uplink is fully power controlled. Subsequently, the analysis is expanded to take into account the path loss and the fractional power control policy when a user is distributed uniformly within a circle of unit radius centered at its serving $\mathrm{BS}$, Finally based on these derivations, the analytical framework is expanded furthermore to be able to quantify the expected outage complexity for a Cloud-RAN composed of $N_{\text {cloud }} \overline{\mathrm{BS}}$ s.

\subsubsection{Full Power Control}

Consider a single-user with a fixed average $\mathrm{SNR} \bar{\gamma}$, and that this user is processed locally (i.e., $N_{\mathrm{c}}=1$ ). The instantaneous value of $\gamma$ is a random variable, which, for the sake of tractability, we assume is an exponential random variable corresponding to the widely accepted Rayleigh fading model ${ }^{4}$. The $\mathrm{PDF}$ of such a $\gamma$ is given by:

$$
f_{\gamma}(\gamma)=\frac{1}{\bar{\gamma}} \exp \left(-\frac{\gamma}{\bar{\gamma}}\right), \gamma \geq 0
$$

\section{Expected complexity}

Theorem 5.4.1 The expected value of the decoding complexity conditioned on the average SNR $\bar{\gamma}$ can be evaluated by

$$
\mathbb{E}_{\gamma \mid \bar{\gamma}}\left\{\mathcal{C}_{i}\right\} \approx \sum_{k=1}^{N_{R}} \frac{\mathcal{R}\left(\gamma_{k}^{R}, \Delta \gamma\right)}{\log _{2}(\zeta-1)}\left[\mathcal{I}_{1}\left(\gamma_{k+1}^{R}, \gamma_{k}^{R}\right)+\mathcal{I}_{2}\left(\gamma_{k+1}^{R}, \gamma_{k}^{R}\right)\right]
$$

where the functions $\mathcal{I}_{1}(\cdot, \cdot)$ and $\mathcal{I}_{2}(\cdot, \cdot)$ are given respectively by (E.3) and E.13).

Proof: The details of the derivation are given in Appendix 5.1.

\footnotetext{
${ }^{4}$ Note that other distributions for $\gamma$ may be used, in order to more accurately model the effects of interference, shadowing, and signal propagation, but this comes at the cost of reduced tractability. For instance the results from Cap. 2 could be used, but such distributions will require a simulation or numerical integration to compute the performance metrics.
} 


\section{Variance of the complexity}

Theorem 5.4.2 The variance of the computational complexity conditioned on the average SNR $\bar{\gamma}$ can be evaluated by

$$
\operatorname{Var}_{\gamma \mid \bar{\gamma}}\left\{\mathcal{C}_{i}\right\} \approx \sum_{k=1}^{N_{R}}\left[\frac{2 \mathcal{R}\left(\gamma_{k}^{R}, \Delta \gamma\right)}{\log _{2}(\zeta-1)}\right]^{2} \mathcal{I}_{4}\left(\gamma_{k+1}^{R}, \gamma_{k}^{R}\right)-\mathbb{E}_{\gamma \mid \bar{\gamma}}\left\{\mathcal{C}_{i}\right\}^{2}
$$

where $E_{\gamma \mid \bar{\gamma}}\left\{\mathcal{C}_{i}\right\}$ is evaluated using (5.15) and $\mathcal{I}_{4}(\cdot, \cdot)$ is given by (E.31).

Proof: The details of the derivation are given in the Appendix 5.2.

\section{Outage complexity}

In the case of an isolated user processed locally, the outage complexity in a distributed system is the value $\mathcal{C}_{\text {outage }}$ that satisfies the following expression

$$
\mathbb{P}\left\{\mathcal{C}_{i} \geq \mathcal{C}_{\text {outage }}\left(\hat{\epsilon}_{\text {comp }}, 1\right)\right\} \leq \hat{\epsilon}_{\text {comp }}
$$

which can be evaluated once the CDF of the complexity is known.

The CDF of the complexity can be written as follows

$$
F_{\mathcal{C}}\left(\mathcal{C}_{\text {thr }}\right)=\mathbb{P}\left\{\mathcal{C}_{i} \leq \mathcal{C}_{\text {thr }}\right\}
$$

where $\mathcal{C}_{\text {thr }}$ is a complexity threshold chosen based on the quality of the channel and the computational resources available. By using the notion that the complexity monotonically decreases in $\gamma$ in the interval $\left[\gamma_{k}^{R} ; \gamma_{k+1}^{R}\right], 5.18$ can be replaced by

$$
F_{\mathcal{C}}\left(\mathcal{C}_{\text {thr }}\right)=\frac{1}{\mathbb{P}\left\{\gamma>\gamma_{1}^{R}\right\}} \sum_{k=1}^{N_{R}} \mathbb{P}\left\{\gamma_{k, \text { min }} \leq \gamma<\gamma_{k+1}^{R}\right\}
$$

where

$$
\begin{aligned}
\gamma_{k, \min } & =\max \left[\gamma_{k}^{R}, \min \left(\gamma_{k+1}^{R}, 2^{\Delta R+r(\gamma, \Delta \gamma)}-1\right)\right] \\
\Delta R & =2^{-\mathcal{C}_{\mathrm{thr}} \log _{2}(\zeta-1) /[2 r(\gamma, \Delta \gamma)]} \sqrt{\frac{\zeta-2}{K \zeta}}
\end{aligned}
$$

Finally, (5.19) can be written as follows

$$
F_{\mathcal{C}}\left(\mathcal{C}_{\text {thr }}\right)=\frac{1}{1-F_{\gamma}\left(\gamma_{1}^{R}\right)} \sum_{k=1}^{N_{R}}\left[F_{\gamma}\left(\gamma_{k+1}^{R}\right)-F_{\gamma}\left(\gamma_{k, \text { min }}\right)\right]
$$

where $F_{\gamma}(\cdot)$ is the $\mathrm{CDF}$ of $\gamma$ with $\mathrm{PDF}$ given by (5.14). 


\subsubsection{Effect of Path Loss and Power Control}

This subsection considers the case that the user is subject to a fractional power control policy and is located randomly within the cell. For the sake of tractability ${ }^{5}$, assume a circular cell of unit radius and that the user is placed according to a uniform distribution. Indicate with $Y_{i}$ both the $i^{\text {th }} \mathrm{BS}$ and its location. Each $\mathrm{BS} Y_{i}$ serves only one user, which is located at $X_{i}$ and is $\left\|Y_{i}-X_{i}\right\|$ away from it.

Under a fractional power control policy and using a distance dependent attenuation power law, as in Cap. 2, $X_{i}$ will transmit using power

$$
P_{i}=P_{0}\left\|Y_{i}-X_{i}\right\|^{\alpha s}
$$

The average SNR received by $Y_{i}$ for the $i^{t h} \mathrm{UE}$ is given by

$$
\bar{\gamma}_{i}=\bar{\gamma}_{\mathrm{ud}}\left\|Y_{i}-X_{i}\right\|^{-\alpha(1-s)}
$$

where $\bar{\gamma}_{\text {ud }}$ is the average reference $\underline{\text { SNR, }}$, measured assuming unit-distance transmission.

In this case, it is not possible to determine a closed-form expression or a suitable approximation for both the expected and the variance of the complexity. However, knowing that the $\mathrm{PDF}$ of $r_{i}=\left\|X_{i}-Y_{i}\right\|$ is

$$
f_{r_{i}}(\omega)= \begin{cases}2 \omega & , 0 \leq \omega \leq 1 \\ 0 & , \text { otherwise }\end{cases}
$$

the expected complexity $\mathbb{E}\left\{\mathcal{C}_{i}\right\}$ and variance $\operatorname{Var}\left\{\mathcal{C}_{i}\right\}$ can be obtained by performing a numerical integration over $r$ of (5.15) for the expected complexity and $(5.16)$ for the complexity variance.

However, under this scenario, the CDF of $\gamma$ averaged over the spatial distribution can be evaluated in closed form. By removing the conditioning over the spatial distribution, it follows that

$$
F_{\gamma}(\gamma)=\int F_{\gamma}(\gamma \mid \omega) f_{r_{i}}(\omega) \mathrm{d} \omega
$$

\footnotetext{
${ }^{5} \mathrm{~A}$ more complicated model for the placement of the user may be used, such as one that accounts for the actual shape of the Voronoi region. While more accurate, this comes at the cost of tractability and will typically require simulation or numerical integration.
} 
where $F_{\gamma}(\gamma \mid \omega)=1-\exp \left(-\frac{\gamma}{\gamma}\right), \gamma \geq 0$ and $\bar{\gamma}$ is given by 5.24. Substituting 5.25 into (5.26) yields

$$
\begin{aligned}
F_{\gamma}(\gamma) & =\int_{0}^{1}\left[1-\exp \left(-\frac{\gamma}{\gamma_{\mathrm{ud}}} \omega^{\alpha(1-s)}\right)\right] 2 \omega \mathrm{d} \omega \\
& =1-2 \int_{0}^{1} \exp \left(-\frac{\gamma}{\gamma_{\mathrm{ud}}} \omega^{\alpha(1-s)}\right) \omega \mathrm{d} \omega .
\end{aligned}
$$

After few algebraic manipulations and using the change of variable $t=\frac{\gamma}{\gamma_{\mathrm{ud}}} \omega^{\alpha(1-s)}$, it yields

$$
F_{\gamma}(\gamma)=1-\frac{2\left(\frac{\gamma}{\gamma_{\mathrm{ud}}}\right)^{-2 /[\alpha(1-s)]}\left[\Gamma\left(\frac{2}{\eta(1-s)}, \frac{\gamma}{\gamma_{\mathrm{ud}}}\right)-\Gamma\left(\frac{2}{\alpha(1-s)}, 0\right)\right]}{\alpha(1-s)}
$$

where the function $\Gamma(z, x)$ is a gamma incomplete function, which is defined as 112

$$
\Gamma(z, x)=\int_{x}^{\infty} t^{z-1} e^{-t} \mathrm{~d} t .
$$

Since the $\mathrm{CDF}$ of $\gamma$ is equal to (5.28), the outage complexity can be determined analytically. In particular, even if 5.20 cannot be expressed in closed form, well-known algorithms such as bisection, Newton, or gradient-search algorithm can be applied. Furthermore, the average achievable rate can be determined by substituting (5.28) into 5.13.

\subsubsection{Centralized Processing}

Using the previously derived expressions for the expectation and the variance of the complexity, in this section the analytical framework is expanded in order to be able to compute through an approximation the maximum decoding complexity when $N_{\text {cloud }} \overline{\mathrm{BS}}$; are centralized. More specifically, the central limit theorem (Lindeberg/Levy) is used, which yields

$$
\mathcal{C}_{\text {outage }}\left(\hat{\epsilon}_{\text {comp }}, N_{\text {cloud }}\right)=\sqrt{\frac{\operatorname{Var}\left\{\mathcal{C}_{i}\right\}}{N_{\text {cloud }}}} \underbrace{\sqrt{2} Q^{-1}\left(2\left(1-\hat{\epsilon}_{\text {comp }}\right)^{N_{\text {cloud }}}-1\right)}_{\text {inv. norm. CDF }}+\mathbb{E}\left\{\mathcal{C}_{i}\right\}
$$

where $Q^{-1}(\cdot)$ is the inverse error function, which has the following series representation 112

$$
Q^{-1}(x)=\sum_{k=0}^{\infty} \frac{v_{k}}{2 k+1}\left(\frac{\sqrt{\pi}}{2} x\right)^{2 k+1}
$$

where $v_{0}=1$ and

$$
v_{k}=\sum_{m=0}^{k-1} \frac{v_{m} v_{k-1-m}}{(m+1)(2 m+1)}
$$




\subsection{Numerical Verification}

In order to evaluate the validity of the analytical framework derived in Sec. 5.4, in this section numerical results are provided based on the 3GPP LTE standard and a 3GPP LTE decoder. The numerical results are compared against those obtained through the previously derived framework. A typical value for an LTE network of $\hat{\epsilon}_{\text {channel }}=0.1$ is chosen for the channel outage constraint.

Assume all the available resources per cell are allocated to a single user. Furthermore, a full-buffer scenario is assumed: each of the $N_{\text {cloud }} \overline{B S}$; , whose signals are jointly processed at a central cloud-processor, always serves a user over the complete bandwidth. However, 3GPP LTE allows for multi-user opportunistic scheduling such that one TB may carry information from more than one user. When resources are shared among multiple users more diversity and therefore a lower outage complexity per cell can be achieved compared to the case when all the resources are monopolized by a single user. Therefore, this assumption represents a worst-case scenario regarding computational complexity, since it does not allow for any computational diversity within a given cell.

A block Rayleigh fading channel is considered, although in practice a system experiences more channel diversity due to multi-path propagation, and the computational complexity varies from user to user and from $\mathrm{CB}$ to $\mathrm{CB}$. This scenario, in an extreme case, leads the users to experience a sufficient channel diversity for a close-to-AWGN channel, which results in a lower computational gain and diversity.

The outage complexity $\mathcal{C}_{\text {outage }}\left(\hat{\epsilon}_{\text {cloud }}, N_{\text {cloud }}\right)$ is obtained through a simulation campaign. During each trial, $\gamma_{i}$ is drawn from an exponential distribution when we do not consider the path loss, or from a distribution whose CDF is given by (5.28) when the path loss is taken into consideration. The MCS scheme for a given $\gamma_{i}$ is determined according to the value of $\Delta \gamma$ chosen. Each of the $C_{k} \mathrm{CB}$; in the TB is marked as being in a channel outage with probability $\epsilon_{\mathrm{cb}}=1-\left(1-\hat{\epsilon}_{\text {channel }}\right)^{1 / C_{k}}$, which can be precomputed. If any of the $\mathrm{CB}$ are in outage, then the entire TB is declared to be in an outage. If the $r^{\text {th }} \mathrm{CB}$ is in an outage, then $L_{r}(\gamma, \Delta \gamma)=L_{\max }$, which is the maximum number of attempted iterations. Otherwise, $L_{r}(\gamma, \Delta \gamma)$ is determined by drawing a random variable distributed according to the PDF of 
$L_{r}(\gamma, \Delta \gamma)$, which can be precomputed by tracking the error-rate as a function of the number of iterations. Once the decoding complexity is computed, finally the outage complexity is evaluated by (5.7). The outage complexity is obtained by using one million trials when evaluated through simulations.

Fig. 5.3 shows the outage complexity to ensure per-cell outage constraint $\hat{\epsilon}_{\text {comp }}=0.1$ as function of the number of BSs, whose signals are centrally processed. Results in Fig. 5.3(a) consider a scenario characterized by a Rayleigh fading channel with $\bar{\gamma}=10 \mathrm{~dB}$, while in Fig. 5.3(b) a distance dependent path loss model is taken into account, as described in Sec. 5.4.2. For both models, three values of $\Delta \gamma$ are considered. For Fig. 5.3(b), the path loss exponent is fixed to $\alpha=2$, the average SNR at unit distance is set to $\gamma_{\mathrm{ud}}=0 \mathrm{~dB}$, and the compensation factor is $s=0.1$, which is the value reported in [113] that maximizes the sum throughput. Higher values of $s$ would improve fairness at the cost of throughput. In Fig. 5.3, the solid lines are evaluated analytically as derived in Section 5.4.1 and 5.4.2, while the dots are obtained through simulations using the methodology described above. The notches on the right side of each sub-figure show the behavior as $N_{\text {cloud }} \rightarrow \infty$. Fig. 5.3 shows a good agreement for both models between the simulated and the analytical results, emphasizing the validity of the proposed analytical framework.

In Section 5.2.2, the complexity is assumed to be deterministic for a fixed SNR, while in an actual implementation this would be a random variable with expectation $\mathcal{C}(\gamma, \Delta \gamma)$. This additional source of randomness is not considered in the analytical framework, and it is one of the reason for the slight mismatch between the empirical and analytical results. Another reason for the slight mismatch is that a lower-bound on the complexity is not applied: an actual decoder always performs at least one iteration which determines the minimum required complexity. If $\mathcal{C}(\gamma, \Delta \gamma)$ is a random variable, the computational variance would increase. This would consequently cause an increment of the computational gain for a Cloud-RAN, Furthermore, if the lower bound is applied, it causes a slight increment of the expected complexity, even if the variance might decrease, which lead also in this case to a higher computational gain. 


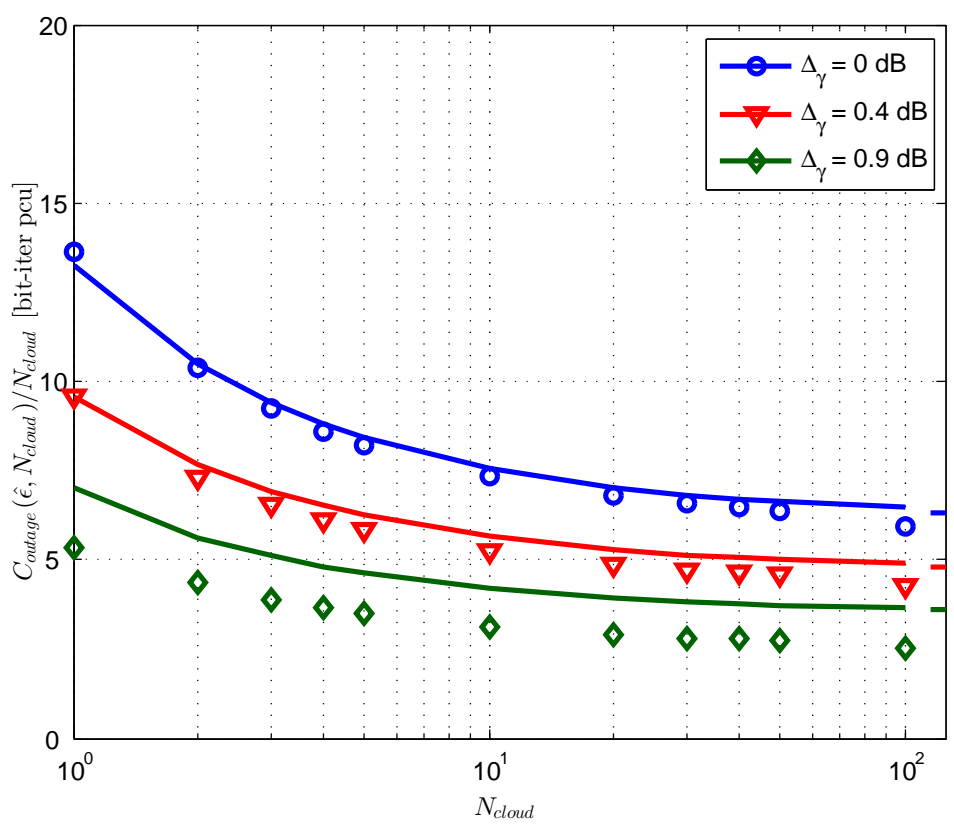

(a) Rayleigh fading

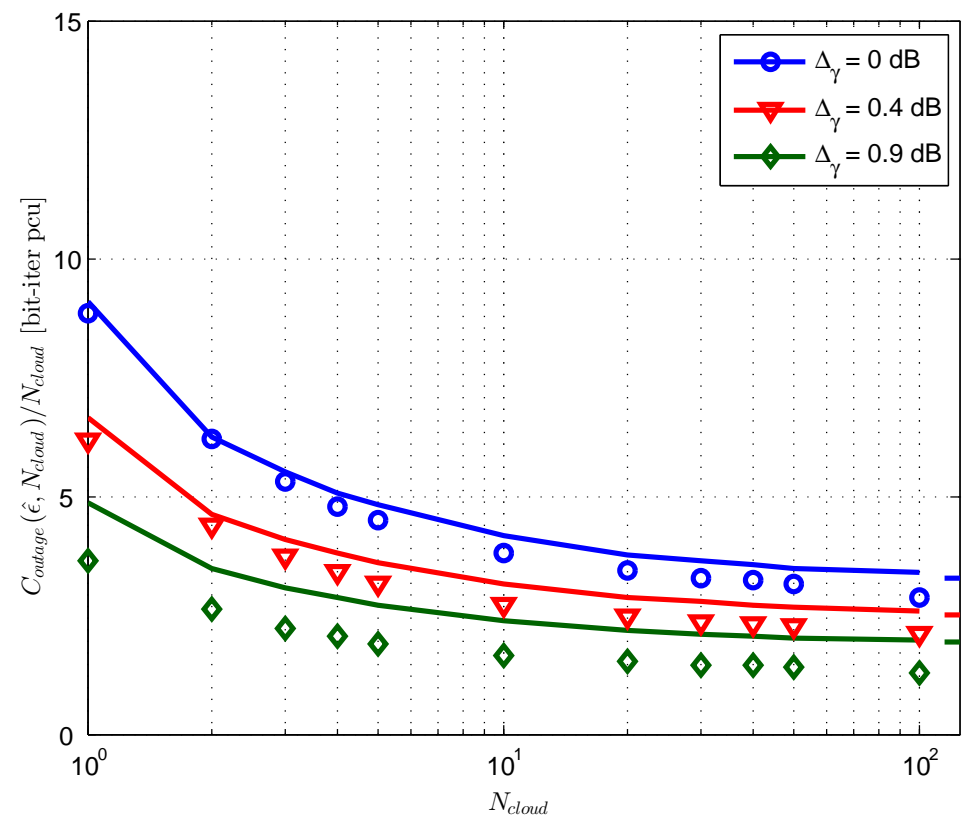

(b) Distance dependent path loss model

Figure 5.3: Outage complexity to ensure per-cell outage constraint $\hat{\epsilon}=0.1$ as function of the number of BSs, whose signals are centrally processed. Solid lines are evaluated analytically, while dots are obtained through simulations using one million trials. The notches on the right side of each sub-figure show the behavior as $N_{\text {cloud }} \rightarrow \infty$. 


\subsection{Results}

In this section, the analytical framework developed in Section 5.4 is applied to perform an evaluation of a centralized system using some of the metrics introduced in Section 5.3 . In the following the path loss exponent is fixed to $\alpha=2$, the compensation factor is $s=0.1$, the per-cell constraint on the computational outage is set to $\hat{\epsilon}_{\text {comp }}=0.1$, and the average SNR at unit distance is $\gamma_{\mathrm{ud}}=0 \mathrm{~dB}$.

\subsubsection{Computational Gain}

At first, the computational gain $g_{\text {comp }}$ and its dependence on the number of centralized BSs and on the SNR margin $\Delta \gamma$ is considered. Fig. 5.4(a) shows the computational gain as function of the number of centralized $\overline{\mathrm{BSS}}$ s. Fig. 5.4(b) shows the computational gain as function of the target computational outage probability.

Fig. 5.4(a) shows that the computational gain increases sharply for small values of $N_{\text {cloud }}$, before it becomes flat for larger values of $N_{\text {cloud }}$. Furthermore, it shows a high computational gain compared to a distributed system, emphasizing the benefit of a centralized solution. The notches on the right side of the sub-figure show the behavior as $N_{\text {cloud }} \rightarrow \infty$.

Fig. 5.4(b) shows that the computational gain increases as the target computational outage probability decreases, since more computational resources are required to make sure that also peak-requirements are satisfied. This is a consequence of the fact that the complexity scales with $-\log _{10}\left(\hat{\epsilon}_{\mathrm{comp}}\right)$ in (5.3). In this case, centralization provides more benefits as diversity effects can be exploited to load balance between the individual BSs: Furthermore, the computational gain differs more significantly for different SNR offsets as the target computational outage probability decreases. This effect is mainly due to the fact that at lower target outage probability it is more challenging to operate close to capacity because the decoder would be required to have a steeper error rate curve.

\subsubsection{Computational Diversity}

Fig. 5.5 shows the computational diversity as function of the target computational outage probability. For each SNR margin $\Delta \gamma$ and target computational outage probability, the 


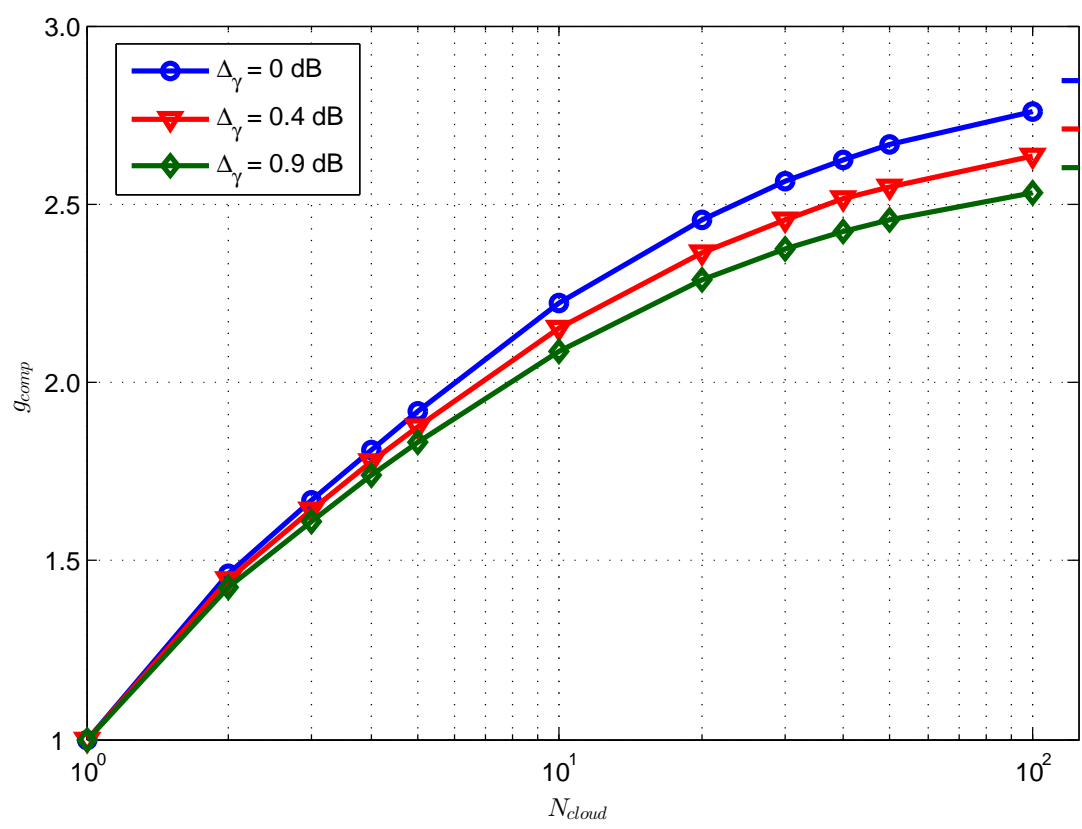

(a) Gain as function of $N_{\mathrm{c}}$

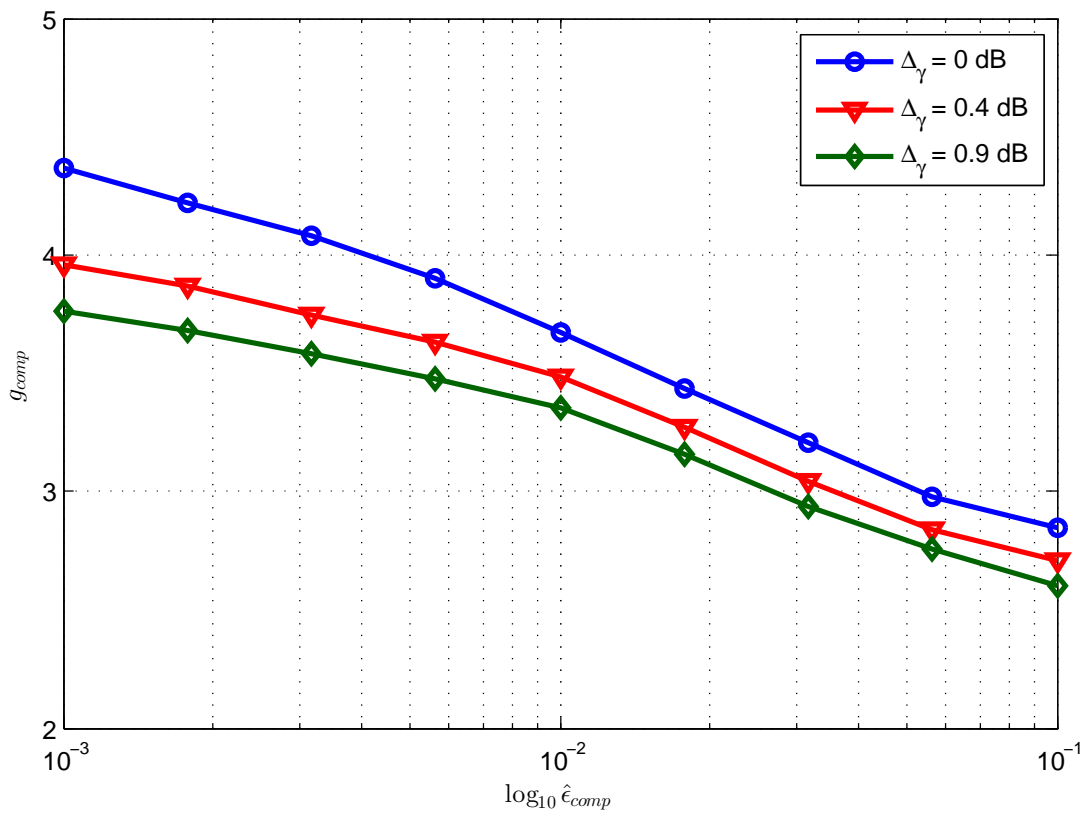

(b) Gain as function of $\hat{\epsilon}$

Figure 5.4: Complexity gain for $\gamma_{\mathrm{ud}}=0 \mathrm{~dB}, \alpha=2, s=0.1$. The notches on the right side of the first sub-figure show the behavior as $N_{\text {cloud }} \rightarrow \infty$. 


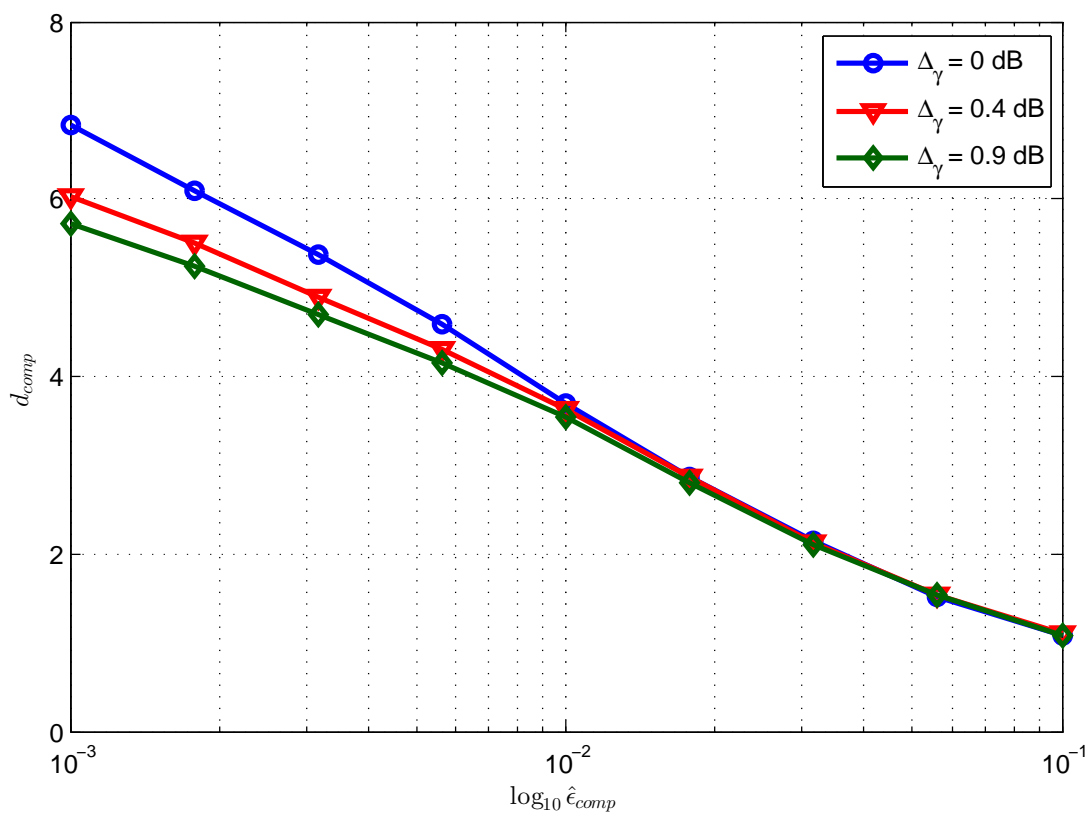

Figure 5.5: Computational diversity as function of the target computational outage probability.

computational diversity is computed by numerically evaluating the derivative in (5.11), once the computational outage probability is obtained from (5.30). The computational diversity, similar to the computational gain, decreases by increasing the target computational outage probability. This is due to the over-provisioning of resources at lower target computational outage probability. Furthermore, the computational diversity differs significantly for different SNR offsets as the target computational outage probability decreases.

\subsubsection{Complexity-Rate Tradeoff}

Fig. 5.6 shows the CRT as function of the number of centralized $\mathrm{BS} s$, which quantifies the increase of the achievable rate as computational resources are added. Fig. 5.6 shows also the dependency of the CRT on the SNR margin offset. The CRT defined in 5.12$)$ is the ratio between the slope of the average rate and the slope of the outage complexity both computed at a given $\mathrm{SNR}$ margin. In Fig. 5.6, the two slopes are individually evaluated numerically using (5.13) and 5.30 for each value of $N_{\text {cloud }}$ and SNR margin $\Delta \gamma$. The notches on the right side of the figure show the behavior as $N_{\text {cloud }} \rightarrow \infty$.

Fig. 5.6 shows that the CRT increases as the margin offset is increased and this is due 


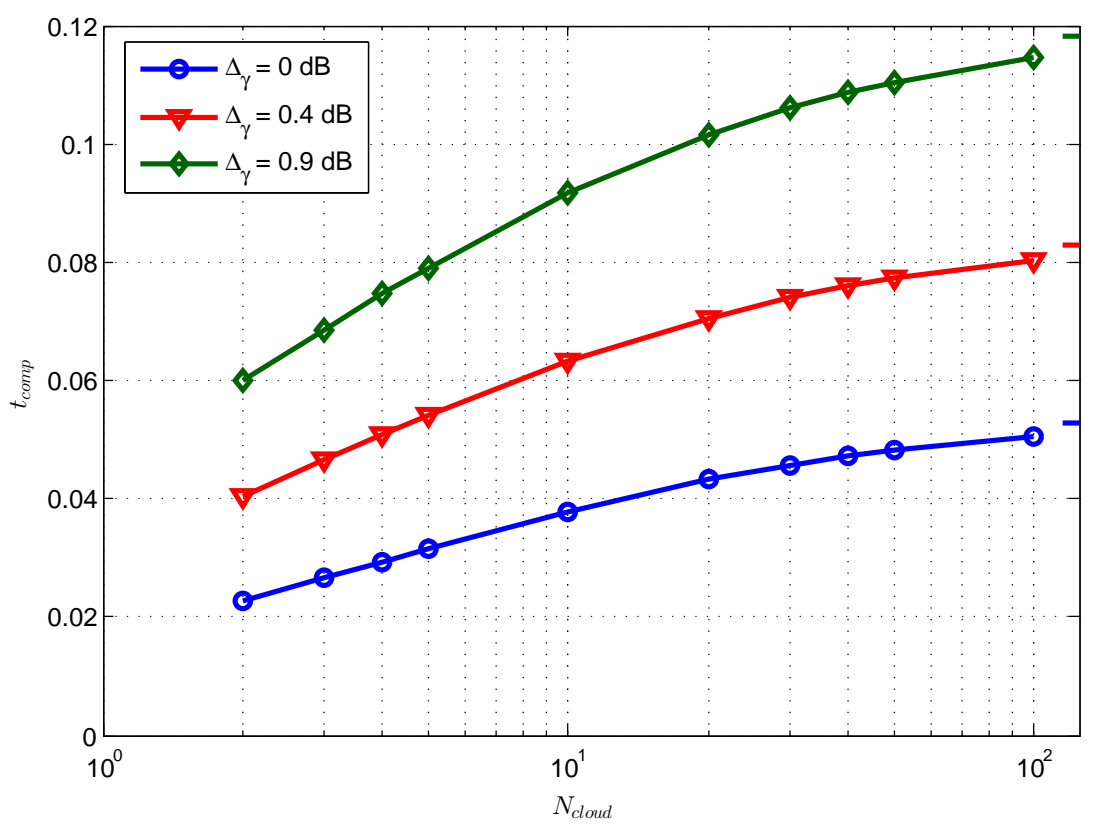

Figure 5.6: CRT as a function of $N_{\mathrm{c}}$. The notches on the right side of the figure show the behavior as $N_{\text {cloud }} \rightarrow \infty$.

to the fact that the decoder operates farther away from capacity and therefore does not cause high peak complexity. Hence, additional complexity is beneficial in order to gain additional achievable rate. In other words, the closer the decoder operates to capacity, the more resources must be invested to increase the achievable rate. Fig. 5.6 points out that an optimum point to operate a system exists. Assume that a minimum average achievable rate is required. In this case, the operator may use a less complex decoder, which reduces the required computational resources (and therefore reduces costs for information technology (IT) infrastructures). However, a minimum computational complexity should be maintained since the penalty in terms of achievable rate may otherwise be significant. In addition, due to the lower per-cell achievable rate, more $\mathrm{BS}$; must be deployed, which implies higher deployment costs. Depending on the costs for $\amalg$ iT infrastructure and mobile network infrastructure, an optimal operating point can be determined where deployment costs are minimized. 


\subsection{Chapter Summary}

Computational limitations in mobile wireless networks have a significant impact on the network performance. Such computational limitations are of particular interest to smallcell networks, where $\mathrm{BS}$ s are computationally limited due to economic constraints. The computational constraints are also of fundamental importance to the design of Cloud-RAN architectures. In this chapter a new analytical framework has been developed to evaluate the data processing requirements of a cellular uplink, which is highly relevant to the analysis and design of Cloud-RAN networks. Several performance metrics have been introduced to quantify the benefit of such a network compared to a conventional RAN: computational outage probability, computational gain, computational diversity and complexity-rate tradeoff. The novel concept of computational outage helps to quantify the complexity-throughput tradeoff in Cloud-RAN platforms. Such a quantification allows for specifying computational requirements for Cloud-RAN platforms in a meaningful way. Furthermore, insights into the computational gain and computational diversity offered by centralized processing are provided. Finally, the complexity-rate tradeoff is used to measure the additional complexity needed to further improve the achievable rate.

The analytical framework has been verified by comparing it numerically with a largescale system level simulator compliant with the 3GPP LTE standard. It has been shown that the analytical framework provides a good match with simulations despite simplifying assumptions, which are necessary for the tractability of the derivations. 


\section{Chapter 6}

\section{Resource Management in Cloud-RANs}

In cellular systems in general, and Cloud-[RAN systems in particular, the amount of computational resources available and the communications performance are strictly related. In fact, a signal can be in outage not only if the channel is not adequate, but also if the computational resources at the $\mathrm{BBU}$ are not enough to its baseband operations. In traditional cellular systems, users must be allocated radio resources to satisfy the objectives of rate, outage, and fairness. However, in a computationally limited environment, such as a CloudRAN, resources should also be allocated with computational constraints in mind. Indeed, the allocation of radio and computational resources are connected, as this will be shown in this chapter. In particular, as the processing load imposed by each device depends on its allocated transmission rate and channel quality, the rate-allocation aspect of the scheduling should take into account the available computing resources.

This chapter provides insights into how the communications performance is effected by the computational resources available, which in modern wireless systems that use capacity approaching codes (i.e turbo or low-density parity-check (LDPC)) are mainly required to perform iterative decoding. In this chapter, it is shown that the overall performance of the network can be improved by adopting a computational aware scheduler, which selects a MCS that requires a reduced number of expected iterations. While this produces a slight loss in terms of instantaneous peak rate, this strategy has the potential to greatly reduce 
the likelihood of computational outages and as a consequence improve the sum-throughput of the system.

This chapter proposes other two computationally aware schedulers, which have the objective of maximizing the sum-rate of the system while satisfying a constraint on the offered computational load. The first scheduler optimally allocates the rates among users and is implemented according to a water-filling algorithm. The second scheduler is suboptimal, but uses a simpler and intuitive complexity-cut-off approach. The performance of both schedulers is evaluated and it is found that both schedulers are able to completely avoid computational outages at the cost of a slight loss of sum-rate.

\subsection{Heuristic Approach to MCS Selection}

As already pointed out in Chap. 5, in a Cloud-RAN system there exists a tradeoff between complexity and throughput. In particular, as a higher achievable rate is sought, a natural choice is to select an MCS that allows the system to work closer to capacity. However, in this case the iterative decoder requires more computational resources to successfully decode the TB, and it is more likely to encounter computational outage. Thus, in modern wireless systems, which uses iterative codes, the iterative nature of the decoding allows complexity to be traded off for transmission rate. This concept is illustrated in Fig. 6.1, which shows the CBLER $\epsilon_{\mathrm{cb}}$ as a function of SNR (expressed as the ratio of the energy-per-symbol to noise power) after 2, 3, and 8 iterations for two MCSs: $I_{\mathrm{mcs}}=10$ and 11. The results in Fig. 6.1 and the remaining examples in this chapter are for an LTE system, with the same specifics provided in Chap. 5. At $\epsilon_{\mathrm{cb}}=2.5 \times 10^{-1}$, the performance of MCS 10 with 2 iterations is the same as the performance of MCS 11 with 8 iterations. Thus, by backing off from MCS 11 to 10, the number of required iterations is cut by a factor of 4, on average. The number of information bits in the $\mathrm{TB}$ that must be decoded is also reduced, in this case from 9216 to 8064 , so the required number of bit-iterations is even further reduced. However, this reduction in complexity comes at a cost, as the spectral efficiency of MCS 10 is lower than that of MCS 11.

The MCS is selected to satisfy a channel outage constraint $\hat{\epsilon}_{\text {channel }}$. When complexity is 


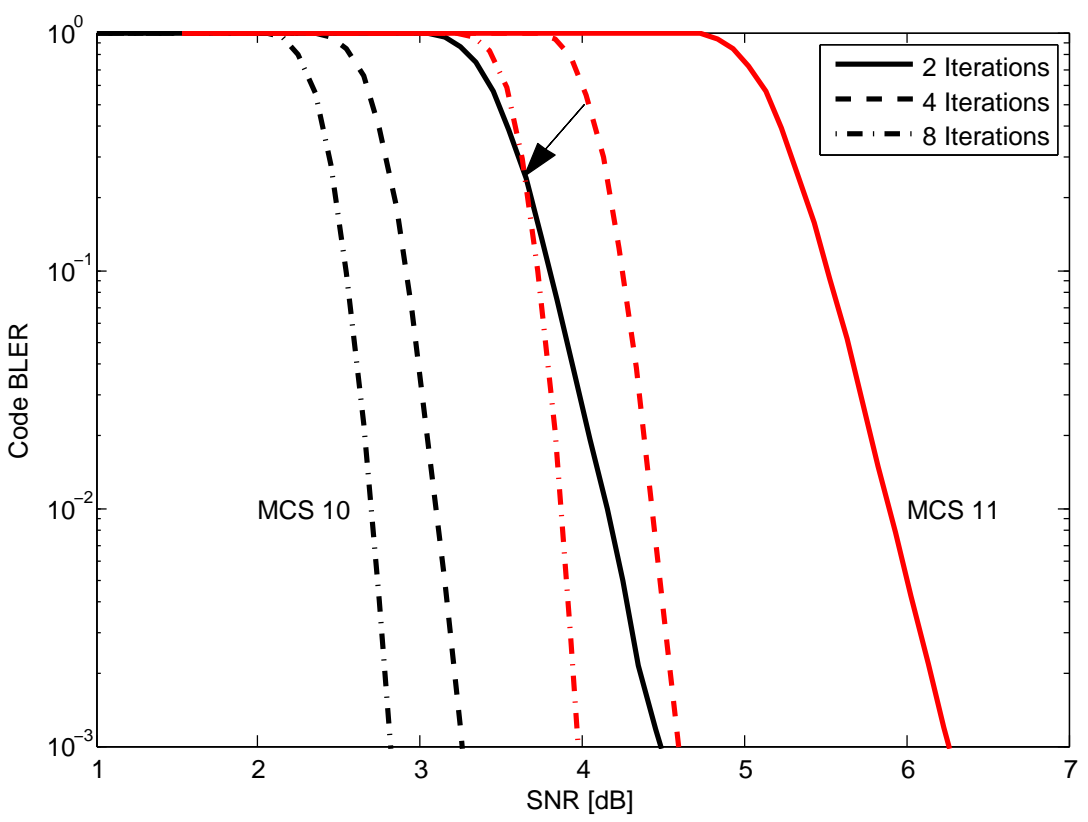

Figure 6.1: Code block error rate as a function of SNR for MCS 10 and MCS 11 after 2, 3, and 8 decoder iterations. The arrow shows where the CBLER of MCS 10 after 2 iterations is the same as that of MCS 11 after 8 iterations.

not a concern, the selected $\mathrm{MCS}$ is the highest one that satisfies an outage constraint $\hat{\epsilon}_{\text {channel }}$ after a large number of decoder iterations. However, when complexity is a concern, the MCS should be selected such that the outage constraint is met after a specific number of decoder iterations. In order to show the benefits in terms of computational outage and throughput when the total complexity requirements are reduced by upper bounding the maximum number of turbo iterations to a lower value, two MCS-selection schemes are defined: i) max-rate selection (MRS) [110], which is able to perform up to $L_{\max }=8$ iterations $(\Delta \gamma=0$ $\mathrm{dB})$; computationally aware selection (CAS) [110], which performs up to $L_{\max }=2$ iterations $(\Delta \gamma=0.9 \mathrm{~dB})$. The rate-complexity tradeoff for these two MCS-selection schemes is illustrated in Fig. 6.2. Fig. 6.2 (a) shows the raw throughput of the MRS and CAS schemes as a function of the $\mathrm{SNR}^{1}$. For reference, the Shannon capacity limit is also shown. The reduction in rate when using CAS is evident from the figure. The average computational effort required to decode a $\mathrm{TB}$ as a function of SNR for the two MCS selection policies is shown in Fig. 6.2(b). As anticipated, using the complexity aware scheme reduces the average effort.

\footnotetext{
${ }^{1}$ The raw throughput $T_{\text {raw }}$ is simply the selected rate $\mathcal{R}(\gamma, \Delta \gamma)$ expressed in units of bits/second. This is in contrast with the effective throughput, which is the rate of correct transmission; i.e, $T_{\text {eff }}=(1-\epsilon) T_{\text {raw }}$.
} 


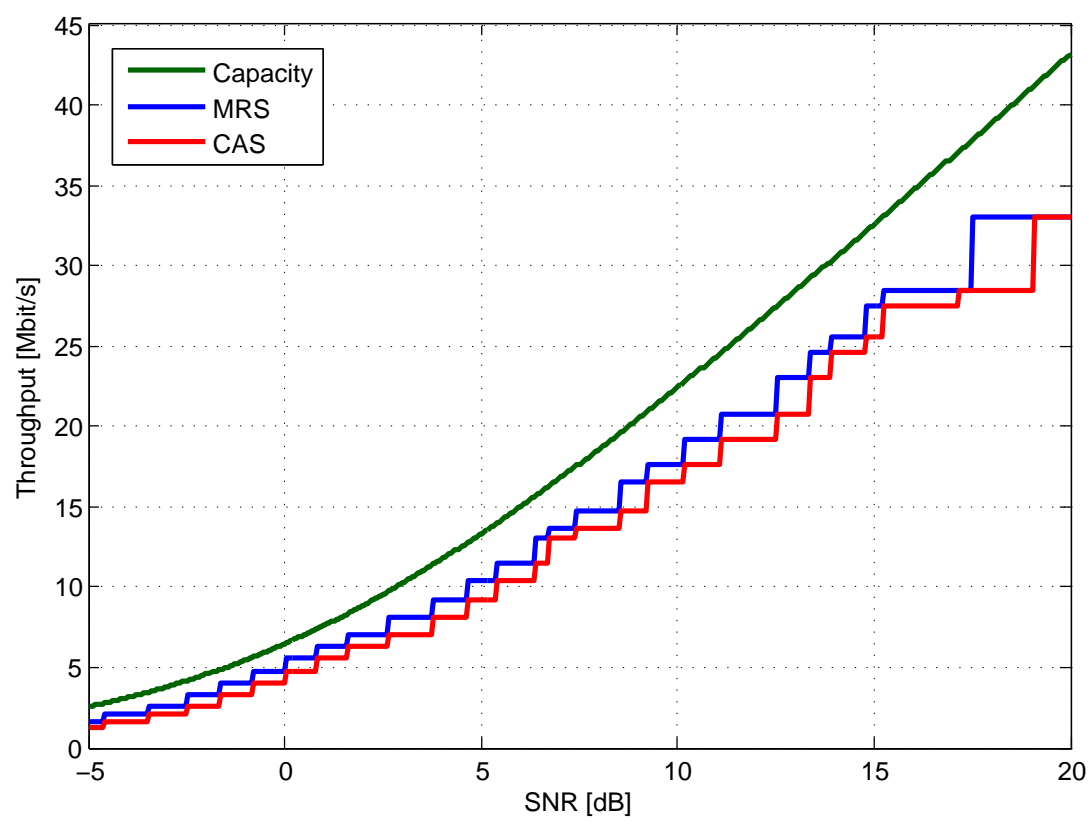

(a) Raw throughput

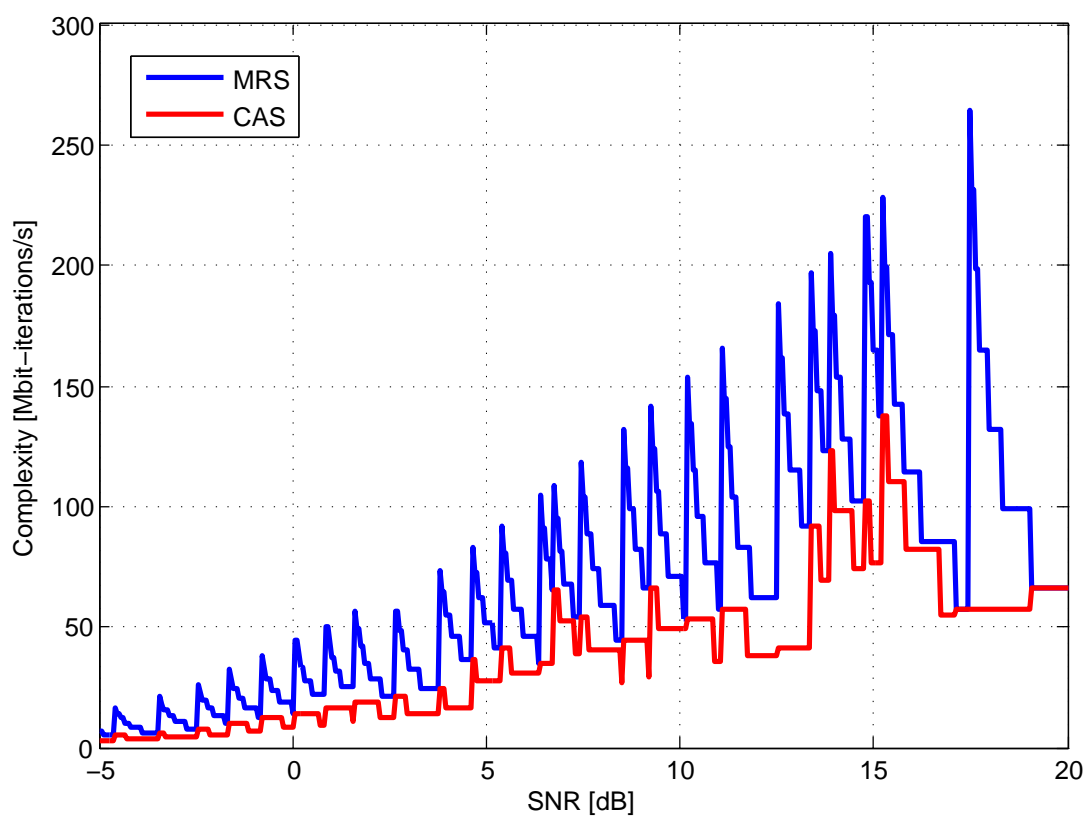

(b) Computational effort

Figure 6.2: Raw throughput and computational effort as a function of the SNR for two MCS selection schemes: computationally aware selection (CAS) and max-rate selection (MRS).

Notice that the stairstep appearance of Fig. 6.2(a) and peaky behavior and Fig. 6.2(b) are due to the use of a finite number of MCSs: 


\subsubsection{Numerical Results}

In this section, the performance of the two aforementioned MCS-selection schemes are analyzed using a system-level simulator that is compliant with the 3GPP LTE standard by accounting for both the channel and the computational outage. In the following, the overall outage probability $\epsilon_{\text {all }}$, or simply outage probability, is in fact the probability that a $\mathrm{TB}$ is lost due to either kind of outage. The performance are first provided in the case of a single cell, followed by the case of a multicell environment where CCT has to be considered.

\section{Outage in an Isolated Cell}

Consider a network with just one cell containing a single active user served by a single BS. There is no interference from other cells, and the signal received by the BS is processed all by itself; hence, $N_{\text {cloud }}=1$. Consider the complexity-throughput tradeoff in a Rayleigh fading channel. The complexity-outage tradeoff is obtained through a simulation that works as follows. During each trial, the $\mathrm{SNR} \gamma$ is drawn from an exponential distribution. The MCS scheme for the given $\gamma$ is determined according to the MCS selection policy. Each of the $C \mathrm{CB}$; in the $\mathrm{TB}$ is marked as being in a channel outage with probability $\epsilon_{\mathrm{cb}}$, which can be precomputed. If any of the $\mathrm{CB}$ are in outage, then the entire $\mathrm{TB}$ is declared to be in an outage. If the $r^{\text {th }} \mathrm{CB}$ is in an outage, then $L_{r}(\gamma, \Delta \gamma)=L_{\max }$, which is the maximum number of attempted iterations. Otherwise, $L_{r}(\gamma, \Delta \gamma)$ is determined by drawing a random variable distributed according to the pdf of $L_{r}(\gamma, \Delta \gamma)$, which can be precomputed by tracking the error-rate as a function of the number of iterations.

Fig. 6.3 shows the outage probability of both MCS-selection schemes in the presence of Rayleigh fading when there is no complexity constraint $\left(\mathcal{C}_{\max }=\infty\right)$ and when there is a complexity constraint of $\mathcal{C}_{\max }=50$ Mbit-iterations per second ${ }^{2}$. As can be seen, using the more conservative CAS scheme greatly reduces the outage probability when complexity is constrained. A non-monotonic behavior is observed in the complexity constrained outage curves. At low SNR, channel outage dominates, as the conservative MCS s that must be

\footnotetext{
${ }^{2}$ The complexity of a typical software turbo decoder requires between 100 and 1,000 floating-point operations per second (FLOPS) for each bit-iteration, depending on the actual implementation [114]. For comparison, modern general-purpose processors support up to 50-200 billion ELOPS,
} 


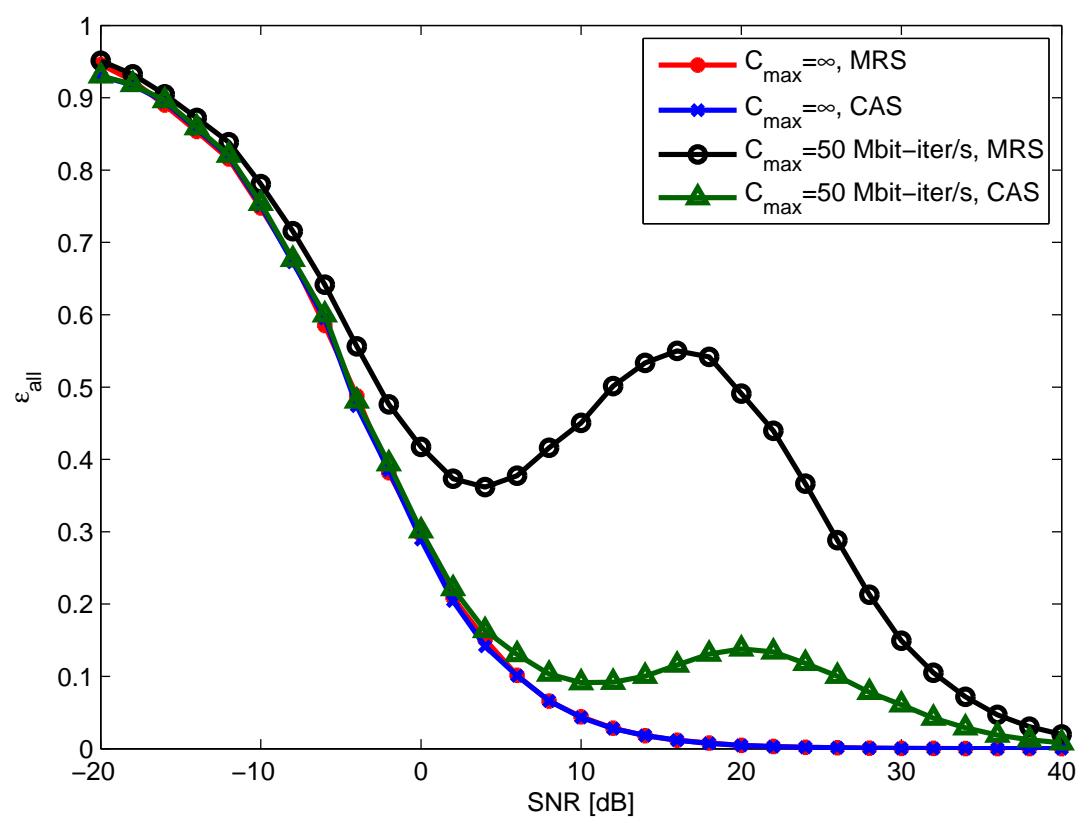

Figure 6.3: Outage probability as a function of average SNR in the presence of Rayleigh fading, both with and without a complexity constraint.

selected do not have particularly high computational requirements due to having few information bits per TB. However, as the average SNR increases, higher MCS frequently and computational outage begins to dominate due to the additional computational burden associated with their larger payloads. However, at very high average SNR, the instantaneous SNR is often much higher than the selection threshold for the highest MCS, and the CB; for that MCS will usually be successfully decoded after just one or two iterations, which is not enough load to trigger a computational outage.

In a complexity constrained system, the advantage of a complexity aware MCS selection is a reduction in outage probability, but its disadvantage is a loss in the raw throughput. However, if the outage probability is sufficiently lower, the effective throughput may be higher despite the reduced raw throughput. This can be seen in Fig. 6.4, which shows the effective throughput as a function of average SNR for the same cases that were shown in Fig. 6.3. The loss in effective throughput due to the complexity constraint is evident in the figure, but much of this loss can be recovered by using complexity aware MCS selection; i.e., the throughput curve for CAS is up to $9 \mathrm{~dB}$ higher than the curve for MRS. 


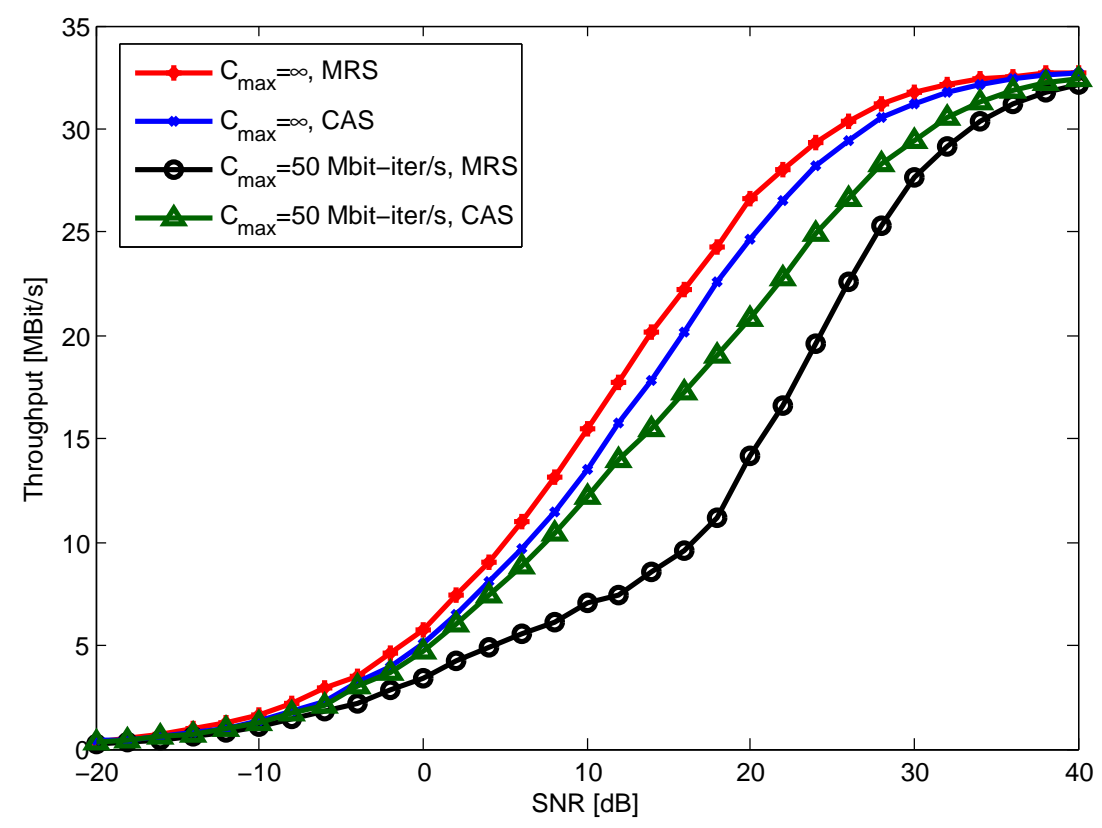

Figure 6.4: Effective throughput as a function of average SNR in the presence of Rayleigh fading, both with and without a complexity constraint.

\section{Outage in a Network}

Now consider a network with $N_{\text {total }}$ BSs, where $N_{\text {total }}$ could be arbitrarily large. Unlike the single-cell scenario, there is co-channel interference on the uplink due to active UEs in adjacent cells. For illustrative purposes, consider the network with $N_{\text {total }}=129$ shown in Fig. 6.5, which is a segment of an actual deployment by a major provider in the UK at 1800 $\mathrm{MHz}$. Furthermore, the processing of the $N_{\text {cloud }}=8$ cells highlighted in yellow is considered. Two options are examined: i) centralized processing $(\underline{C P})$, where the signals from all $N_{\text {cloud }}$ cells are processed in the cloud; ii) and local processing $(\underline{L P})$, where the signals at each $\mathrm{BS}$ are locally processed.

Assume that the UE; are distributed according a $\mathrm{PPP}$ with intensity $\lambda$ users per $\mathrm{km}^{2}$. BSS $Y_{i}$ serves a cell with area $\left|\mathcal{A}_{i}\right|$. The number of $\overline{\mathrm{UE}}$; in $Y_{i}$ 's cell is a Poisson variable with mean $\lambda\left|\mathcal{A}_{i}\right|$. While the SC-FDMA uplink channel is partitioned into a plurality of $\mathrm{RB}$; that could be allocated to different users, similar to Chap. 5, it is assumed that all of the available resources of a particular subframe are allocated to a single UE. When there is more than one user in $Y_{i}^{\prime}$ 's cell, then one of the users is selected at random to transmit on the uplink. The likelihood of a transmitting user in $Y_{i}$ 's cell is the complement of the void probability. 


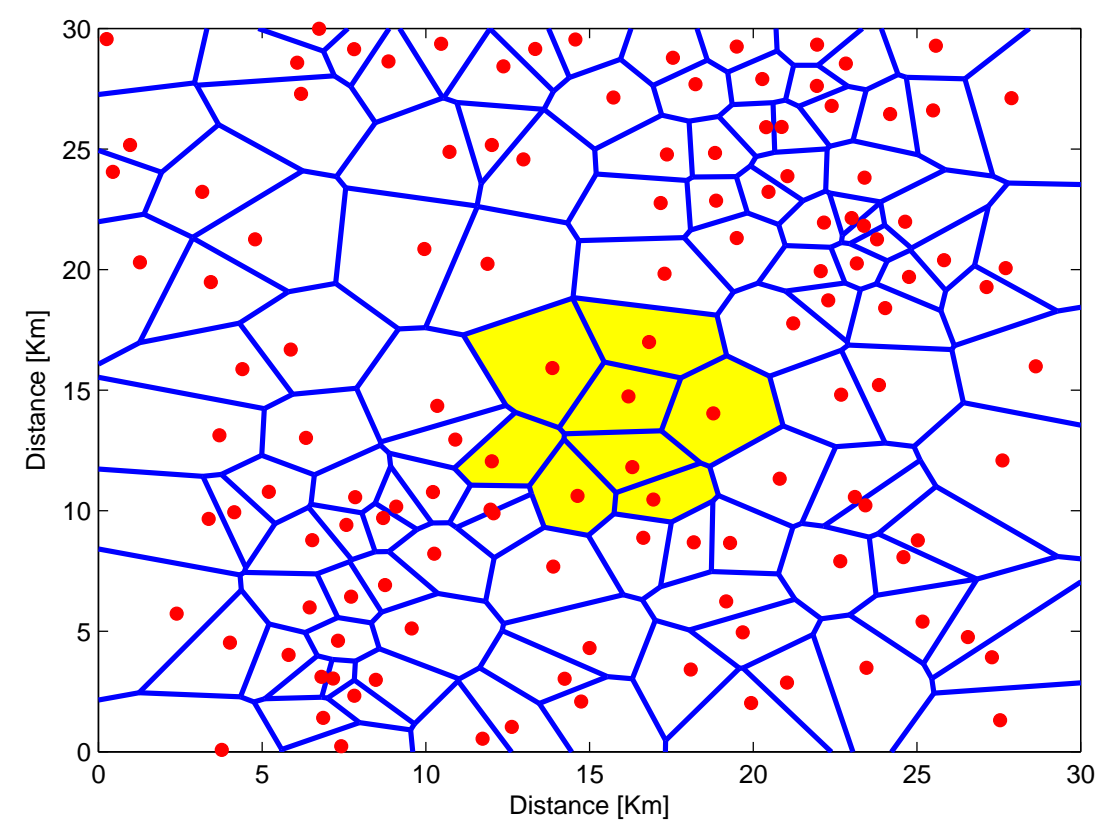

Figure 6.5: Base station locations. The cloud group consists of the highlighted cells in the center of the diagram.

Thus, there will be a $\mathrm{UE}$ transmitting to $Y_{i}$ with probability $1-\exp \left(-\lambda\left|\mathcal{A}_{i}\right|\right)$, and when there is a transmission, the location of the user is uniform within the cell. The location of the UE transmitting to $Y_{i}$ is denoted by $X_{i}$. Fractional power control policy is adopted, and it is assumed that $s=0.1$. For this scenario,

$$
\gamma_{j}=\frac{g_{j, j}\left|Y_{j}-X_{j}\right|^{\alpha(s-1)}}{\mathrm{SNR}^{-1}+\sum_{i \neq j} g_{i, j}\left|Y_{j}-X_{i}\right|^{-\alpha}\left|Y_{i}-X_{i}\right|^{s \alpha}} .
$$

As in the single-cell case described in Section 6.1.1, the throughput is determined with the assistance of a Monte Carlo simulation. During each trial, which corresponds to a subframe, a $\mathrm{UE}$ is placed at random in the $i^{\text {th }}$ cell with probability $1-\exp \left(-\lambda\left|\mathcal{A}_{i}\right|\right)$. Once the $\mathrm{UE} ;$ are placed, the fading coefficients $\left\{g_{i, j}\right\}$ are drawn from an exponential distribution and the SINR at each BS in the cloud group is computed according to (6.1). The TB; that are in a channel outage are identified and the computational effort $C\left(\gamma_{i}, \Delta \gamma\right)$ for each TB is determined as before.

Both $\mathrm{LP}$ and $\mathrm{CP}$ are considered. In the $\mathrm{LP}$ case, if $C\left(\gamma_{i}, \Delta \gamma\right)>\mathcal{C}_{\max }$ for a given TB, then it is considered to be in an outage. For the CP case, if $(5.6)$ is violated, then an outage occurs in at least one of the uplink $\overline{\mathrm{TB}} \mathrm{s}$. Scheduling in the virtual $\mathrm{BS}$ pool is assumed to 


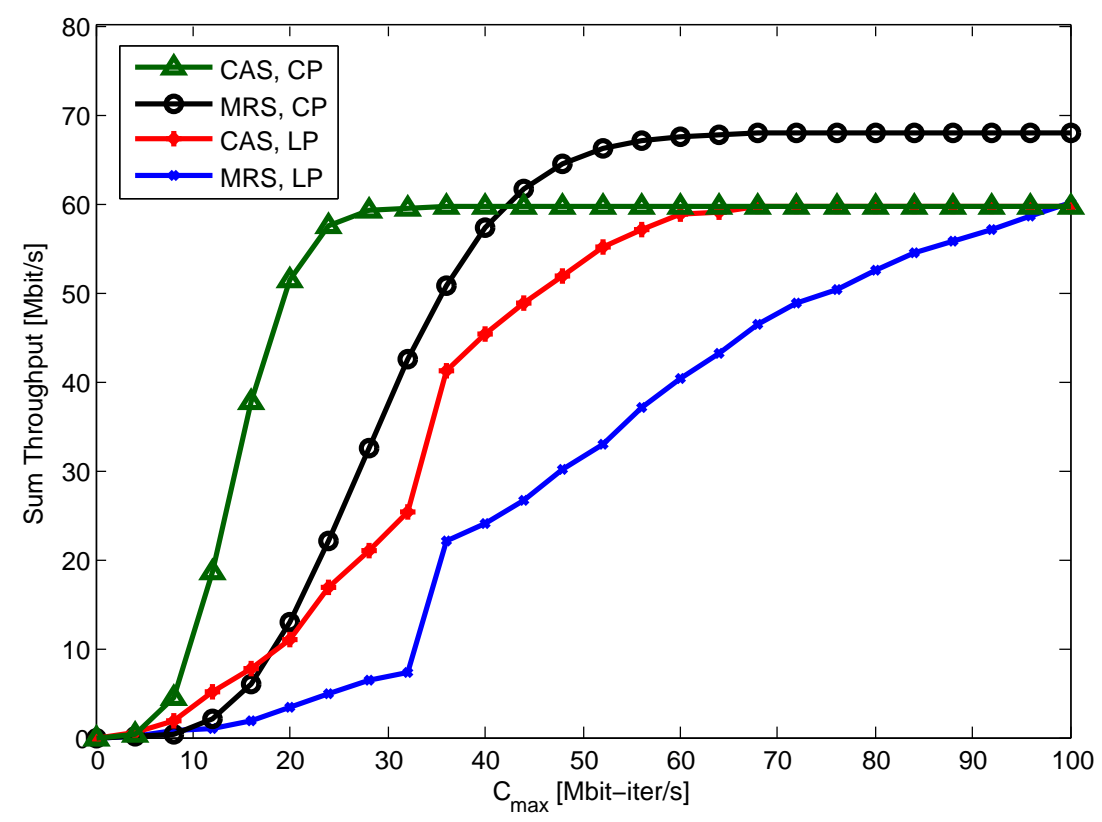

Figure 6.6: Sum throughput as function of the per-BS complexity constraint when $N_{\text {cloud }}=8$, with local processing (LP) and with cloud processing (CP). Two MCS-selection schemes are considered: computationally aware selection (CAS) and max-rate selection (MRS).

process the signals with low SINR first. This allows those TB that are sent at a low MCS, and hence have a lower complexity requirement, to be first decoded, and the TB; that fail are the ones that were sent at the highest MCS:

Fig. 6.6 shows the sum throughput, which is the sum of the effective throughputs in each cell of the cloud group, as a function of the per- $\overline{\mathrm{BS}}$ complexity constraint $\mathcal{C}_{\max }$ with the two MCS-selection policies. The plot was generated assuming a UE density of $\lambda=0.1$, path loss exponent $\alpha=3.7$, and average SNR SNR $=20 \mathrm{~dB}$. As can be seen, $\mathrm{CP}$ is much more computationally efficient than $\mathrm{LP}$. For the same complexity constraint, the throughput of $\mathrm{CP}$ is significantly higher than it is with $\mathrm{LP}$. The computational diversity advantage of CP is evident from its steeper curve. Alternatively, the complexity constraint required to achieve a desired throughput is much lower for $\mathrm{CP}$ than it is for $\mathrm{LP}$. These behaviors confirm the computational diversity benefits of cloud processing. Furthermore, there is a benefit to using the more conservative CAS policy for the LP system. For the CP system, CAS is beneficial as long as $\mathcal{C}_{\max } \leq 40 \mathrm{Mbit}-i t e r / \mathrm{s}$; for systems with less of a complexity constraint MRS is better.

Fig. 6.7 shows the effect of UE density $\lambda$ on the sum throughput. Again, $\alpha=3.7$, and the 


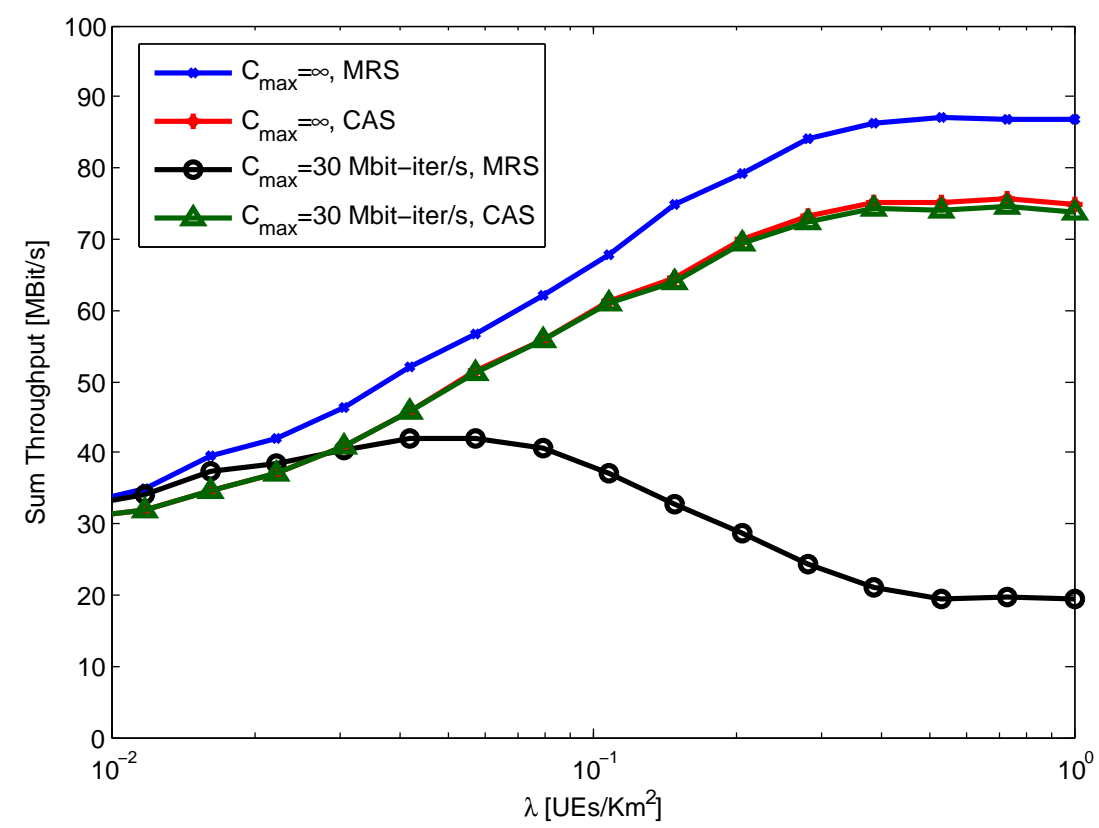

Figure 6.7: Sum throughput with cloud processing as function of the density of UEs when $N_{\text {cloud }}=8$. Two MCS-selection schemes are considered: computationally aware selection (CAS) and max-rate selection (MRS).

average $\underline{\mathrm{SNR}} \Gamma=20 \mathrm{~dB}$. The figure shows results for $\mathrm{CP}$, both with no complexity constraint $\left(\mathcal{C}_{\max }=\infty\right)$ and with a constraint of $\mathcal{C}_{\max }=30 \mathrm{Mbit}$-iter $/ \mathrm{s}$. The two MCS selection policies are again considered. When there is no complexity constraint, the sum throughput rises with $\lambda$. In this case, the throughput of MRS is higher than CAS, However, when there is a complexity constraint, throughput falls with higher values $\lambda$ when MRS is used. This is due to a high prevalence of computational outages in heavily loaded systems. However, if the more conservative CAS policy is used, performance is approximately the same as if there were no complexity constraint. This confirms the effectiveness of using a more conservative MCS-selection policy in dense networks.

\subsection{Optimal MCS Scheduling Policies}

The CAS scheduler presented in the previous section was heuristically designed with no claims of optimality. This section takes a more formal approach to the derivation of a scheduler with the objective of maximizing the sum-rate under complexity constraints. This section derives in Sec. 6.2.1 an optimal water-filling scheduling policy when the rates are 
drawn from a continuum of possible rates. Next, in Sec. 6.2.2 the optimization is modified to account for the more practical case of having an MCS scheme with only finite number of possible rates to pick from. In Sec. 6.2.3, an alternative low-complexity resource-allocation strategy is proposed that is based on an intuitive complexity cut-off approach. Finally, this section concludes by evaluating the performance of the proposed schedulers using a systemlevel simulator, which is complaint with the 3GPP LTE standard.

\subsubsection{Max-Rate Optimization Problem}

Let $\boldsymbol{R}_{i}=\left\{R_{1}, \ldots R_{N_{c}}\right\}$ be the set of rates allocated to each uplink for the $i^{\text {th }}$ resource allocation strategy. Then, the set $\boldsymbol{R}^{*}=\left\{\boldsymbol{R}_{1}, \ldots \boldsymbol{R}_{N_{A}}\right\}$ with $N_{A} \leq N_{R}^{N_{c}}$ denotes the set of all feasible rate allocations.

Suppose that the Cloud-RAN platform supports a maximum computational complexity such that $\sum_{k} \mathcal{C}_{k} \leq N_{\text {cloud }} \mathcal{C}_{\text {max }}$. Further, assume that the set of allocated rates may be drawn from a continuum of possibilities. Our objective is to maximize the sum-rate of the system while avoiding any computational outage, which can be formalized as follows:

$$
\begin{aligned}
& \boldsymbol{R}_{\mathrm{opt}}=\arg \max _{\boldsymbol{R} \subset \boldsymbol{R}^{\prime}} \sum_{R_{k} \in \boldsymbol{R}} R_{k}, \\
& \text { s.t. } \sum_{R_{k} \in \boldsymbol{R}} \mathcal{C}_{k}\left(\gamma_{k}, \Delta \gamma\right) \leq N_{\text {cloud }} \mathcal{C}_{\text {max }} \text {. }
\end{aligned}
$$

where $\mathcal{C}_{k}\left(\gamma_{k}, \Delta \gamma\right)$ is given by $(5.3)$ and $\boldsymbol{R}^{\prime}$ is the set of all rate allocations satisfying $0 \leq$ $R_{k} \leq \log \left(1+\gamma_{k}\right)$ for all $k$.

This scheduling problem implies a trade-off between the invested computational complexity and the gained achievable rates. In order to solve (6.2), the partial derivative $\partial \mathcal{C}_{k}\left(\gamma_{k}, \Delta \gamma\right) / \partial R_{k}$ is required, which determines how much the computational complexity varies when the data rate is changed. However, the derivative of $\mathcal{C}_{k}\left(\gamma_{k}, \Delta \gamma\right)$ is rather complex and difficult to apply in this optimization problem. Let recall the term $l_{k}\left(\gamma_{k}, R_{k}\right)$ defined in Appendix 5.1, and let use that fact that from 5.1) $\mathcal{R}\left(\gamma_{k}^{R}, \Delta \gamma\right)=R_{k}$, then

$$
l_{k}\left(\gamma_{k}, R_{k}\right)=\log _{2}\left(1+\gamma_{k}\right)-R_{k}
$$


Apply a piecewise linearization to $(6.3)$, yields

$$
l\left(\gamma_{k}, R_{k}\right) \approx e_{k} R_{k}+g_{k}
$$

where

$$
\begin{aligned}
e_{k} & =\frac{\partial l\left(\gamma_{k}, R_{k}\right)}{\partial R_{k}}=\frac{-1}{\log (2)\left[\log _{2}\left(1+\gamma_{k}\right)-R_{k}\right]} \\
g_{k} & =\log _{2}\left[\log _{2}\left(1+\gamma_{k}\right)-R_{k}\right]-e_{k} R_{k} .
\end{aligned}
$$

Using (6.4), we can rewrite (5.3) as

$$
\mathcal{C}_{k}\left(\gamma_{k}, \Delta \gamma\right)=v_{k} R_{k}^{2}+o_{k} R_{k}
$$

where

$$
\begin{aligned}
v_{k} & =-\frac{2 e_{k}}{\log _{2}(\zeta-1)} \\
o_{k} & =\frac{1}{\log _{2}(\zeta-1)}\left[\log _{2}\left(\frac{\zeta-2}{K\left(\hat{\epsilon}_{\text {channel }}\right) \zeta}\right)-2 g_{k}\right] .
\end{aligned}
$$

Theorem 6.2.1 The solution to the rate allocation problem in (6.2) can be well approximated by

$$
R_{k}=\frac{1}{2 v_{k}}\left(\frac{1}{\eta}-o_{k}\right)^{+}
$$

where $0 \leq \eta \leq 1 / o_{k}$ and $\sum_{R_{k} \in \boldsymbol{R}} \mathcal{C}_{k}\left(\gamma_{k}, \Delta \gamma\right) \leq N_{\text {cloud }} \mathcal{C}_{\text {max }}$.

The proof follows similar arguments and methodology of the power allocation problem [115], which leads to the well known water-filling method.

The details of the derivation are provided in Appendix $F$.

In (6.6) the parameter $1 / \eta$ determines the water-level, which decides whether a UE is served or not, while $o_{k}$ it is the cost (in terms of complexity) of transmitting. If the difference between the selected rate $R_{k}$ and the capacity $\log \left(1+\gamma_{k}\right)$ is small, then the term $o_{k}$ becomes very large (due to the large slope of $e_{k}$ ). Hence, the UE is unlikely to be served. The parameter $v_{k}$ scales the rate, i.e. if again the rate $R_{k}$ operates close to capacity, $v_{k}$ will be also very large and therefore scales down the assigned data rate in order to reduce the necessary computational complexity. 


\subsubsection{Application to Limited Number of Rates and Multiple Users}

The solution of $(6.2)$ provided by Theorem 6.2.1 is not practical in the more realistic case that the allocated rate must be drawn from among a finite set of discrete MCSlevels. Assume now that the allocation is over the set $\boldsymbol{R}^{*}$ of feasible discrete allocations. The discretized allocation may be stated as:

$$
\begin{aligned}
R_{k} & \stackrel{(a)}{=} \frac{1}{2 v_{k}}\left(\frac{1}{\eta}-o_{k}\right) \\
& \stackrel{(b)}{\geq} \sqrt{\frac{\mathcal{C}_{k}\left(\gamma_{k}, \Delta \gamma\right)}{v_{k}}+\left(\frac{o_{k}}{2 v_{k}}\right)^{2}}-\frac{o_{k}}{2 v_{k}}
\end{aligned}
$$

where $(a)$ follows from Theorem 6.2.1 assuming that $0 \leq \eta \leq 1 / o_{k}$ and $(b)$ results from 6.5), assuming there is no constraint on the computational complexity.

Combining (6.7) and (6.8), it can be further stated that

$$
\begin{aligned}
\frac{1}{\eta} & \geq 2 v_{k} \sqrt{\frac{\mathcal{C}_{k}\left(\gamma_{k}, \Delta \gamma\right)}{v_{k}}+\left(\frac{o_{k}}{2 v_{k}}\right)^{2}} \\
& =\sqrt{4 v_{k} \mathcal{C}_{k}\left(\gamma_{k}, \Delta \gamma\right)+o_{k}^{2}}
\end{aligned}
$$

which gives the required water-level for each user.

Following from $(6.10)$ and $($ F.8 $)\left(\eta>1 / o_{k} \Longrightarrow R_{k}=0\right)$, yields

$$
\sqrt{4 v_{k} \mathcal{C}_{k}\left(\gamma_{k}, \Delta \gamma\right)+o_{k}^{2}} \geq o_{k} \Longrightarrow R_{k}=0
$$

which is a condition that makes sure that the computational resources released are invested to those users, which can be served with higher assignable rates.

Another drawback of $(6.2)$ is that it does not assign the computational resources in a fair manner. In particular, the aforementioned solution always favors those users that have a higher SINR and it allows them to transmit at the highest rates, as can be seen from function $l_{k}(\cdot, \cdot)$, while the other users might be dropped.

In order to schedule the rates more fairly from the set of allowed values, the following iterative procedure can be used:

1. Initialization: 
(a) Set $\boldsymbol{R}$ such that each user $k$ receives the maximum possible rate $R_{k}$.

(b) Set $R_{k}=0$ for all users for which (6.11) is satisfied.

2. Recursion: If $\sum_{R_{k} \in \boldsymbol{R}} \mathcal{C}_{k}\left(\gamma_{k}, \Delta \gamma\right)>N_{\text {cloud }} \mathcal{C}_{\max }$ then

(a) Compute 6.10 for each user and select $k^{*}$ user with the highest value: $k^{*}=$ $\operatorname{argmax}_{k} \sqrt{4 \alpha \mathcal{C}_{k}\left(\gamma_{k}, \Delta \gamma\right)+o_{k}^{2}}$.

(b) Decrease the rate for user $k$ to the next lower MCS and update $\boldsymbol{R}$ accordingly.

3. Decision:

(a) Halt the process if $\sum_{R_{k} \in \boldsymbol{R}} \mathcal{C}_{k}\left(\gamma_{k}, \Delta \gamma\right) \leq N_{\text {cloud }} \mathcal{C}_{\text {max }}$. Otherwise, go back to step 2 ,

4. Attempt to serve dropped users:

(a) Find the users for which $R_{k}=0$ and among them find $k^{*}$ such that $k^{*}=$ $\operatorname{argmax}_{k} \sqrt{4 \alpha \mathcal{C}_{k}\left(\gamma_{k}, \Delta \gamma\right)+o_{k}^{2}}$.

(b) Assign to the user $k^{*}$ the rate provided in 1.a and update $\boldsymbol{R}$ accordingly.

(c) Halt the process if $\sum_{R_{k} \in \boldsymbol{R}} \mathcal{C}_{k}\left(\gamma_{k}, \Delta \gamma\right) \geq N_{\text {cloud }} \mathcal{C}_{\text {max }}$, otherwise go back to 4 a, If

$$
\sum_{R_{k} \in \boldsymbol{R}} \mathcal{C}_{k}\left(\gamma_{k}, \Delta \gamma\right)>N_{\text {cloud }} \mathcal{C}_{\text {max }}
$$

set $R_{k^{*}}=0$, and update $\boldsymbol{R}$ accordingly.

In the previously described method, step 1-3 iteratively reduce the MCS for the user with the highest value of $(6.10)$, until the complexity constraint is satisfied. Step 4 attempts to assign eventual remaining computational resources to those users that were dropped even though their MCS were sufficiently high. In the following, this process is called scheduling with water-filling (SWF) [116].

\subsubsection{Complexity Cut-Off}

This sub-section introduces a slightly simpler and intuitive scheduling method. Instead of applying (6.6), which requires the linearization described in (6.4) in order to determine 
the parameters $v_{k}$ and $o_{k}$, we select the users with the highest complexity and reduce their MCS (and associated rate $R_{k}$ ) until the sum-complexity constraint $N_{\text {cloud }} \mathcal{C}_{\text {max }}$ is fulfilled. In this case an iterative method, similar to the one described in Sec. 6.2 .2 can be used, which works as follows:

1. Initialization: Set $\boldsymbol{R}$ such that each user $k$ receives the maximum possible rate $R_{k}$,

2. Recursion: If $\sum_{R_{k} \in \boldsymbol{R}} \mathcal{C}_{k}\left(\gamma_{k}, \Delta \gamma\right)>N_{\text {cloud }} \mathcal{C}_{\max }$ then

(a) Select the user $k^{*}$ with the highest complexity: $k^{*}=\operatorname{argmax}_{k} \mathcal{C}_{k}\left(\gamma_{k}, \Delta \gamma\right)$.

(b) Decrease the rate for user $k$ to the next lower MCS and update $\boldsymbol{R}$ accordingly.

3. Decision: Halt the process if $\sum_{R_{k} \in \boldsymbol{R}} \mathcal{C}_{k}\left(\gamma_{k}, \Delta \gamma\right) \leq N_{\text {cloud }} \mathcal{C}_{\text {max }}$. Otherwise, go back to step 2 .

As the result of this procedure, a rate allocation $\boldsymbol{R} \subset \boldsymbol{R}^{*}$ is obtained, which satisfies the complexity constraint and always reduces the rate of those users that require the higher complexity. The main difference to the previously discussed allocation algorithm is that this is not necessarily sum-rate optimal. However, it is still very efficient as it is discussed in the following of this section. In the following, this method is called scheduling with complexity cut-off (SCC) 116 .

\subsubsection{Numerical Results}

In this sub-section, the performance of the proposed rate allocation strategies are compared in terms of complexity and sum-rate. Furthermore, the performance of SWF and SCC are compared against MRS, The comparisons are made by using a system-level simulator that is compliant with the 3GPP LTE standard. In the following, it is assumed $L_{\max }=8$ and the complexity model parameters $\left\{\zeta, K^{\prime}, \nu\right\}$ are chosen as in Chap. 5 for an objective channel constraint $\hat{\epsilon}_{\text {channel }}=0.1$. For illustrative purposes, consider the same network used in Sec. 6.1.1 and shown in Fig. 6.5. Again it is assumed block Rayleigh fading, the UE are distributed according to a $\mathrm{PPP}$ with intensity $\lambda$, a fractional power control policy is adopted 
with $s=0.1$, a distance-dependent path loss model is used with $\alpha=3.7$, and average SNR $\mathrm{SNR}=20 \mathrm{~dB}$.

The sum-rate and the sum-complexity of the system are used in the following as performance metrics to compare the proposed allocation strategies with MRS. Both performance metrics are evaluated through simulations for each of the allocation strategies under examination as follows. During each trial, a UE is placed at random in the $k^{\text {th }}$ cell with probability $1-\exp \left(-\lambda\left|\mathcal{A}_{k}\right|\right)$. Once the UEs are placed, the fading coefficients are drawn from an exponential distribution and the $\mathrm{SINR}$ at each of the $N_{\text {cloud }} \overline{\mathrm{BSS}}$; is computed. By applying a given allocation strategy, we find the selected $\mathrm{MCS}$ for each $\mathrm{BS}$ and the corresponding rate based on the quality of the channel. Using (5.3), the complexity required to process the uplink signal of each $\mathrm{UE}$ is evaluated. The sum-rate and sum-complexity are now computed by summing up respectively the rates and complexity for all $N_{\text {cloud }} \mathrm{BS} s$. Once the allocation algorithm is applied, if $\sum_{k} \mathcal{C}_{k}\left(\gamma_{k}, \Delta \gamma\right)>N_{\text {cloud }} \mathcal{C}_{\text {max }}$, a computational outage occurs and the sum-rate is set to zero.

Fig. 6.8 shows the CDF; of both the achieved sum-rate and the required computational complexity over $N_{\text {cloud }}=8$ BSs. The figure shows the curves for both $\mathrm{SWF}$ and $\mathrm{SCC}$, when the system is designed such that $\epsilon_{\text {comp }}=10 \%, 1 \%, 0.1 \%$ computational outage holds (the notches in the magnification of Fig. 6.8(a) show the corresponding value of $N_{\text {cloud }} \mathcal{C}_{\max }$ ). Fig. 6.8 shows as a benchmark the curve for the unconstrained case, which selects the maximum possible rate that can be used since there is no computational constraint. Fig. 6.8(a) shows that a stronger constraint on the available computational resources implies higher computational outage. More importantly, this figure highlights that while the required computational complexity is significantly reduced by dimensioning the system to a higher computational outage, the sum-rate only decreases slightly for both [SWF and [SCC, i. e. the average sum-rate only decreases by $\approx 0.28 \%$ for $\epsilon_{\text {comp }}=10 \%$ and by $\approx 0.07 \%$ for $\epsilon=0.1 \%$.

This shows the efficiency of the proposed schedulers, which impact the achievable sumrate only marginally, while they reduce the required computational resources significantly. Furthermore, Fig. 6.8 shows that the introduced schedulers are able to completely avoid any computational outage, which would lead in the worst case to drop the connection to the UE: Even if one solution could be to dimension the system for a very low computational outage, 


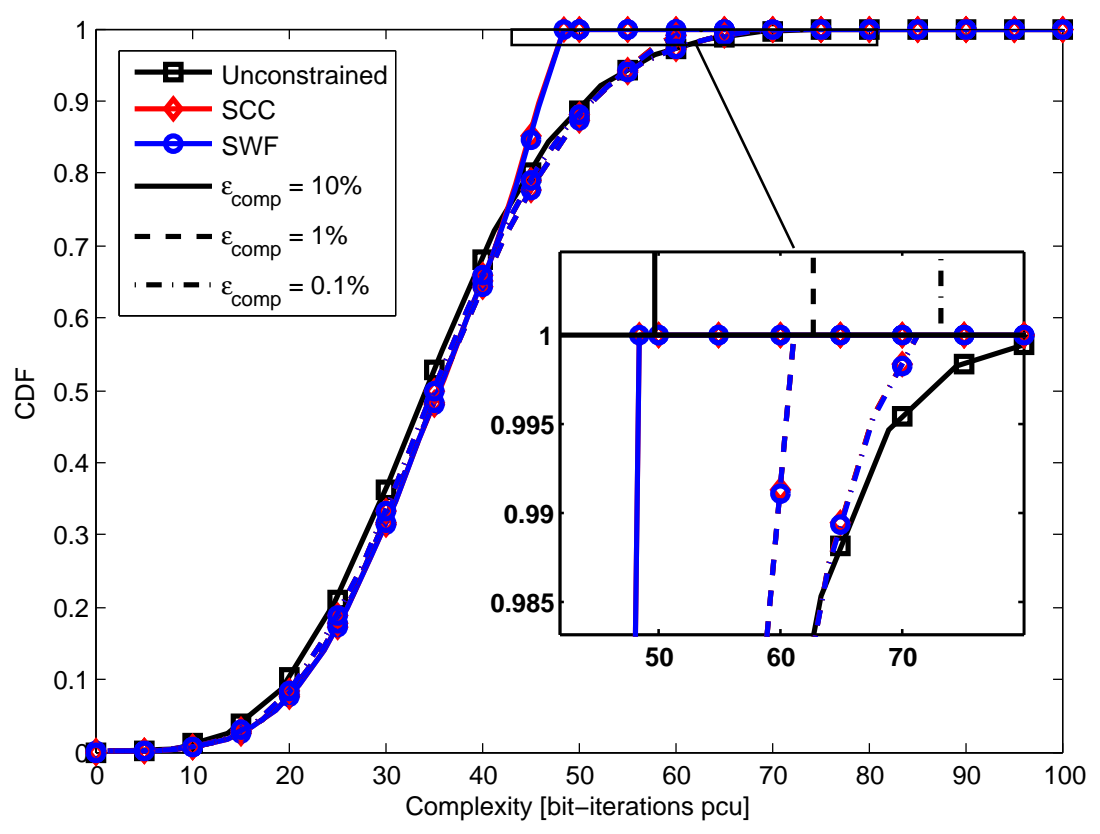

(a) CDF of the computational complexity

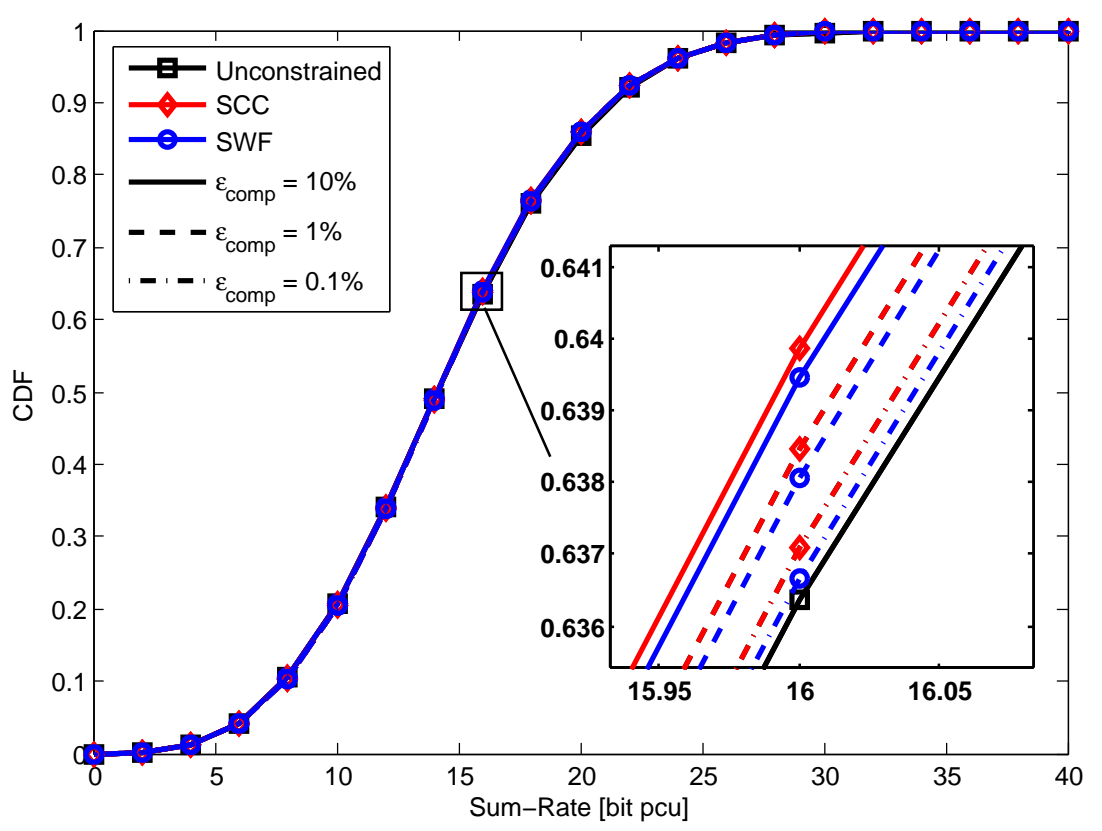

(b) CDF of the sum-rate

Figure 6.8: The system is designed such that $\epsilon_{\text {comp }}=10 \%, 1 \%, 0.1 \%$ and the curves are indicated by solid, dashed, and dash-dotted lines, respectively. The vertical lines in the magnification of Fig. 6.8(a) show the corresponding value of $N_{\text {cloud }} \mathcal{C}_{\max }$ used.

i. e. $\epsilon_{\text {comp }}=10^{-6} \%$, the drawback is that the system will be significantly over-provisioned and most of the time the allocated resources are under-utilized. By contrast, these proposed 


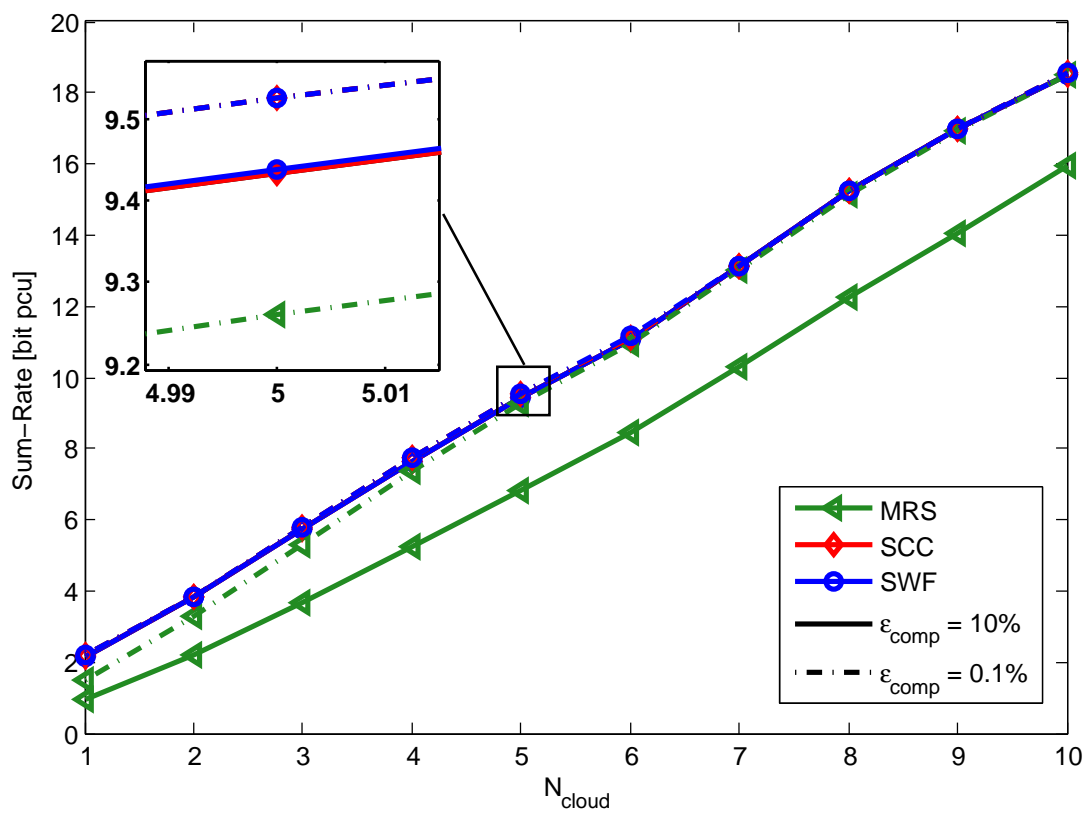

Figure 6.9: Sum-rate as a function of $N_{\text {cloud }}$. The system is designed such that it suffers from $\epsilon_{\text {comp }}=10 \%, 0.1 \%$ computational outage and the curves are indicated by solid, and dash-dotted lines, respectively.

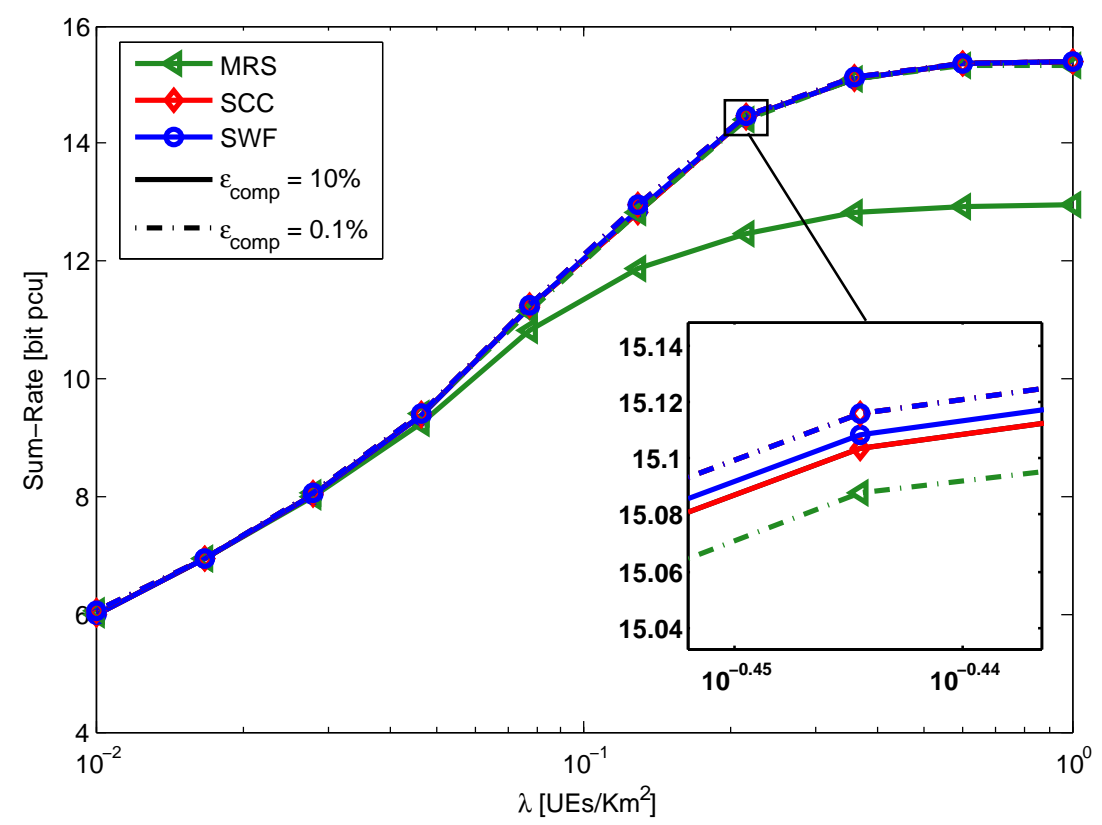

Figure 6.10: Sum-rate as a function of the user-density $\lambda$. The system is designed such that it suffers from $\epsilon_{\text {comp }}=10 \%, 0.1 \%$ computational outage in the case of $\lambda=0.5 \mathrm{UEs} / \mathrm{km}^{2}$. The curves are indicated by solid, and dash-dotted lines, respectively.

schedulers allow to avoid computational outage, while maintaining a high server utilization.

Fig. 6.9 shows the sum-rate as a function of the number of $\overline{\mathrm{BS}}$; , which are centrally 
processed at the Cloud-RAN platform. The performance figures are shown for SWF and SCC as well as for MRS, which does not account for the computational constraint. Fig. 6.9 shows the impact of the computational outage targets on the different schedulers. As it can be noticed, for all values of $N_{\text {cloud }}$, the impact of the constraint on the computational resources is marginal for the computational aware schedulers. By contrast, as $\epsilon_{\text {comp }}$ increases, the impact on a system, which uses MRS, increases linearly with $\epsilon_{\text {comp }}$ due to the increasing computational outage. Furthermore, the magnification in Fig. 6.9 shows that there is only a marginal difference between $\mathrm{SWF}$ and the $\mathrm{SCC}$, emphasizing the fact that the less complex SCC algorithm achieves almost the same performance as SWF

In mobile networks, it may happen that the traffic demand differs significantly over time. In this case the system experiences traffic peaks, e.g. when many people leave or join the metro or an event. This may lead to cases where the system experiences a higher computational outage than it was designed for. Fig. 6.10 shows the sum-rate of SWF, SCC and MRS as function of the density of UE resources available lead to a computational outage of $10 \%$ and $0.1 \%$ in the case of $\lambda=$ $0.5 \mathrm{UEs} / \mathrm{Km}^{2}$. Fig. 6.10 shows the ability of our proposed schedulers to provide services to all the users while slightly reducing the system-throughput. Furthermore, it is shown that the proposed scheduling algorithms are able to accommodate the increasing traffic demand, while the MRS suffers gradually from the higher computational outage.

\subsection{Chapter Summary}

Computational limitations in mobile wireless networks have a significant impact on the network performance. Such computational limitations are of particular interest to smallcell networks, where $\overline{\mathrm{BS}} \mathrm{s}$ are computationally limited due to economic constraints. The computational constraints are also of fundamental importance to the design of Cloud-RAN architectures, and in the management of the resources available.

The first part of this chapter introduced an heuristic selecting policy for MCS selection. By intentionally selecting a lower MCS value, the computational demand of the system is reduced and the effective throughput increases due to a reduction in computational outage. 
The second part of this chapter formulated a new analytical framework, which follows from Chap. 5, and solves the user rate allocation problem under the assumption of limited computational resources in a centralized cloud platform. The underlying optimization problem can be solved with an adapted water-filling approach, making it feasible to fulfill the strict timing requirements of the wireless access. This chapter further shows that an intuitive complexity-cut-off approach delivers near-to-optimal results as well. Finally, the numerical evaluation confirms that meeting computational complexity constraints does not lead to significant penalties in terms of throughput, a fact which highlights the applicability of the approach in practical systems. 


\section{Chapter 7}

\section{Frequency-Hopping: Optimization in Ad Hoc Networks}

The new generation of wireless networks are expected to use a large number of new advanced technologies in order to reduce the deployment costs, decrease power consumption, support the constant and exponential growth of the data traffic, and enhance the overall throughput of the network. In this context, the well known technology of [FH will also play a very important rule, since it can improve the performance of the network by adding an additional degree of diversity that will mitigate the effect of frequency-selective fading and prevent a near-far problem. Furthermore, FH will provide an additional way to further reduce the effect of interference.

This chapter, after providing a brief introduction about this technology and its benefits, introduces the concept of modulation-constrained area spectral efficiency (MASE), which is a form of ASE that accounts for the modulation format used. Two rate allocation policies are considered, and for each one, a methodology is provided for analyzing and optimizing [FH ad hoc networks in the presence of Nakagami fading and shadowing with interfering devices drawn from an arbitrary point process. In this chapter, it is assumed that the system uses a capacity-approaching code (e.g., turbo or LDPC) and non-coherent binary CPFSK modulation, which is the preferred modulation for FH systems. Although CPFSK has a compact spectrum, some of the signal power inevitably splatters into adjacent frequency channels, thereby causing $\mathrm{ACI}$. The amount of $\mathrm{ACT}$ is controlled by setting the fractional in- 
band power; i.e., the fraction of the signal power that lies within the band of each frequency channel, which is often selected arbitrarily.

The chapter first considers the case when a common rate is used among the network. In this case, the optimization is performed with the aim of maximizing the MASE over the number of hopping channels, modulation index, code rate and fractional in-band power, which are jointly optimized for a given channel model and spatial distribution of the interfering devices.

Since in an ad hoc network interferers might move, join, or leave, under the aforementioned scenario there is a potential for wide swings in the outage probability. This results in an unacceptably large delay jitter when a retransmission protocol is used and might lead to outage probabilities that are not acceptable for some modern systems. In order to overcome this issue, while it is not desirable to change the number of frequency-hopping channels, the modulation index, or the fractional in-band power, link adaptation should be performed in an effort to meet a constraint on outage probability. The last part of this chapter focuses on adaptive $\mathrm{FH}$ systems, where the rate is adjusted in order to meet the performance objective, while the modulation format is optimized according with the channel model and spatial distribution of the interfering devices in order to maximize the throughput of the network.

\subsection{Outage Analysis of Frequency-Hopping Networks}

An ad hoc network or peer-to-peer network comprises autonomous systems that communicate without a centralized control or assistance. Ad hoc networks include mobile communication networks that possess no supporting infrastructure, fixed or mobile; usually each device has identical signal processing capability.

[FH spread spectrum is a versatile channel access strategy for ad hoc networks [74. With [FH, a transmitter and its receiver periodically hop within a large set of frequency channels over a given bandwidth according to a specific pseudo-random algorithm. When the transmitter and the receiver remain tuned to the same center frequency, they are able to communicate. $\mathrm{FH}$ can exploit a much wider bandwidth than is possible with a single carrier frequency, and the band can be divided into noncontiguous segments. Fig. 7.1 illustrates the 


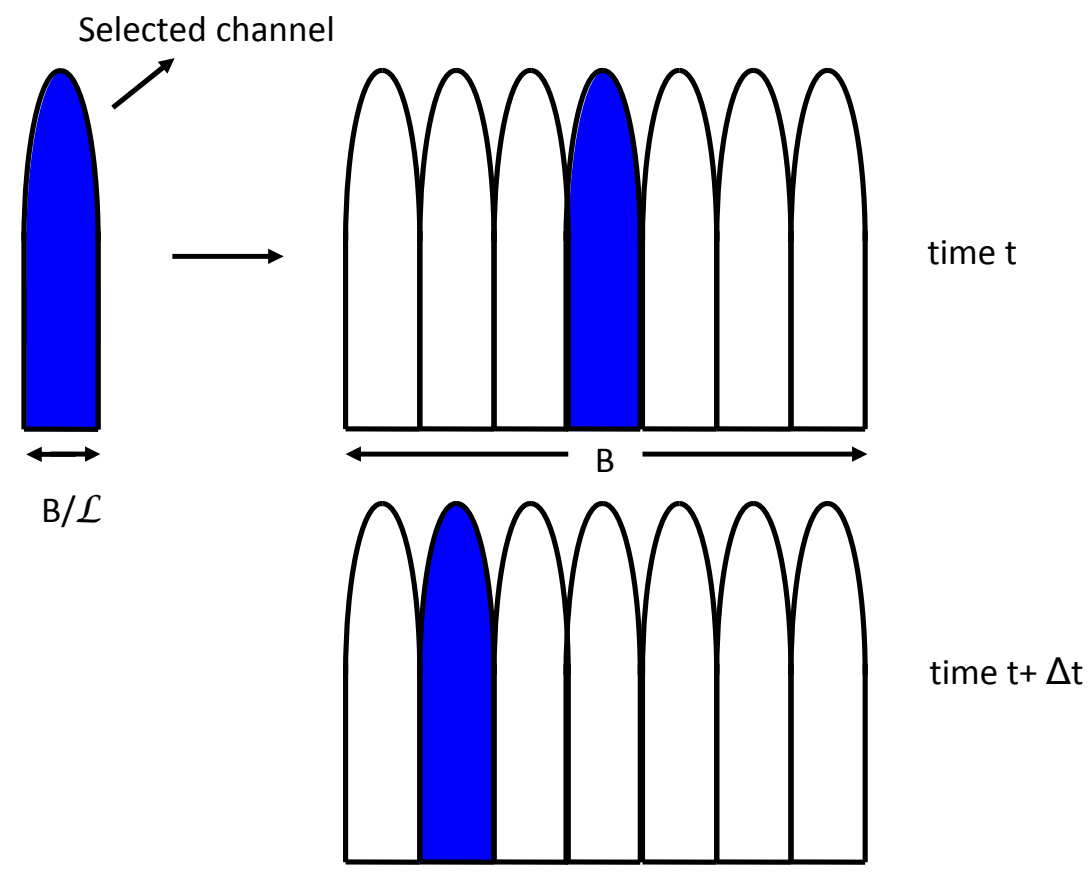

Figure 7.1: Illustration of the bandwidth usage in a FH spread spectrum system.

bandwidth usage for a transmitter in a $\mathrm{FH}$ spread spectrum system, when the bandwidth is divided into $\mathcal{L}=7$ contiguous frequency channels, among which a transmitter switches from time to time to perform a transmission.

Furthermore, $\mathrm{FH}$ provides inherent resistance to the near-far problem, without requiring power control. These advantages [117, 118] are decisive in many applications, and $\mathrm{FH}$ may be added to almost any communication system to strengthen it against interference or fading. Thus, FH may be applied to the set of carriers used in a multicarrier DS-CDMA system or the subcarriers of an OFDMA system. For instance, in the LTE cellular communication system, $[\mathrm{FH}$ is used to randomize the channel assignment from one slot to the next 101 .

\subsubsection{Network Model}

Consider an ad hoc network that use an ALOHA-type multiple-access protocol and CPFSK modulation, which is commonly used for this type of system, since it has a very compact spectrum and produces little spectral splatter. CPFSK modulation is characterized by its modulation order, i.e., the number of possible tones, and by its modulation index $h$, 
which is the normalized tone spacing.

This chapter adopts a similar network model of Sec. 2.1. The network is composed of $M+$ 2 devices, which are distributed within an annular region of outer radius $r_{\text {out }}$ and inner radius $r_{\text {in }}$ : a reference receiver which is located at the center of the arena, a reference transmitter $X_{0}$ and $M$ potential interferers $\left\{X_{1}, \ldots, X_{M}\right\}$. The nonzero $r_{\text {in }}$ models an exclusion zone, which represents the minimum distance to a potential interferer that is imposed by the multipleaccess protocol or constraints on the placement of the devices. The channel access is through a slow frequency-hopping protocol. Assume a distance-dependant power law, and presence of fading and shadowing. In particular, assume that the set of power gains due to fading remains fixed for the duration of a hop, but vary independently from hop to hop. An overall spectral band of $B \mathrm{~Hz}$ is divided into $\mathcal{L}$ contiguous frequency channels, each of bandwidth $B / \mathcal{L} \mathrm{Hz}$. The devices independently select their transmit frequencies with equal probability. The source or reference transmitter $X_{0}$ selects a frequency channel at the edge of the band with probability $2 / \mathcal{L}$ and a frequency channel not at the edge with probability $(\mathcal{L}-2) / \mathcal{L}$. Let $D_{i} \leq 1$ be the duty factor of $X_{i}$, which is the probability that the device transmits any signal.

Two types of collisions are possible: i) co-channel collisions, which involve the source and interfering device selecting the same frequency channel, and ii) adjacent-channel collisions, which involve the source and interfering device selecting adjacent channels. Let $p_{\mathrm{c}}$ and $p_{\mathrm{a}}$ denote the probability of a co-channel collision and an adjacent-channel collision, respectively. Assuming that $D_{i}=D$ is constant for every device, $\mathcal{L}_{e}=\mathcal{L} / D$ denotes the equivalent number of frequency channels. If $X_{i}, i \geq 0$, transmits a signal, then it will select the same frequency as $X_{0}$ with probability $1 / \mathcal{L}$. Since $X_{i}$ transmits with probability $D$, the probability that it induces a co-channel collision is $p_{\mathrm{c}}=D / \mathcal{L}$. If $X_{i}, i \geq 0$, transmits a signal, it will select a frequency channel adjacent to the one selected by $X_{0}$ with probability $1 / \mathcal{L}$ if $X_{0}$ selected a frequency channel at the edge of the band (in which case, there is only one adjacent channel), otherwise it will select an adjacent channel with probability $2 / \mathcal{L}$ (since there will be two adjacent channels). It follows that, for a randomly chosen channel, the 


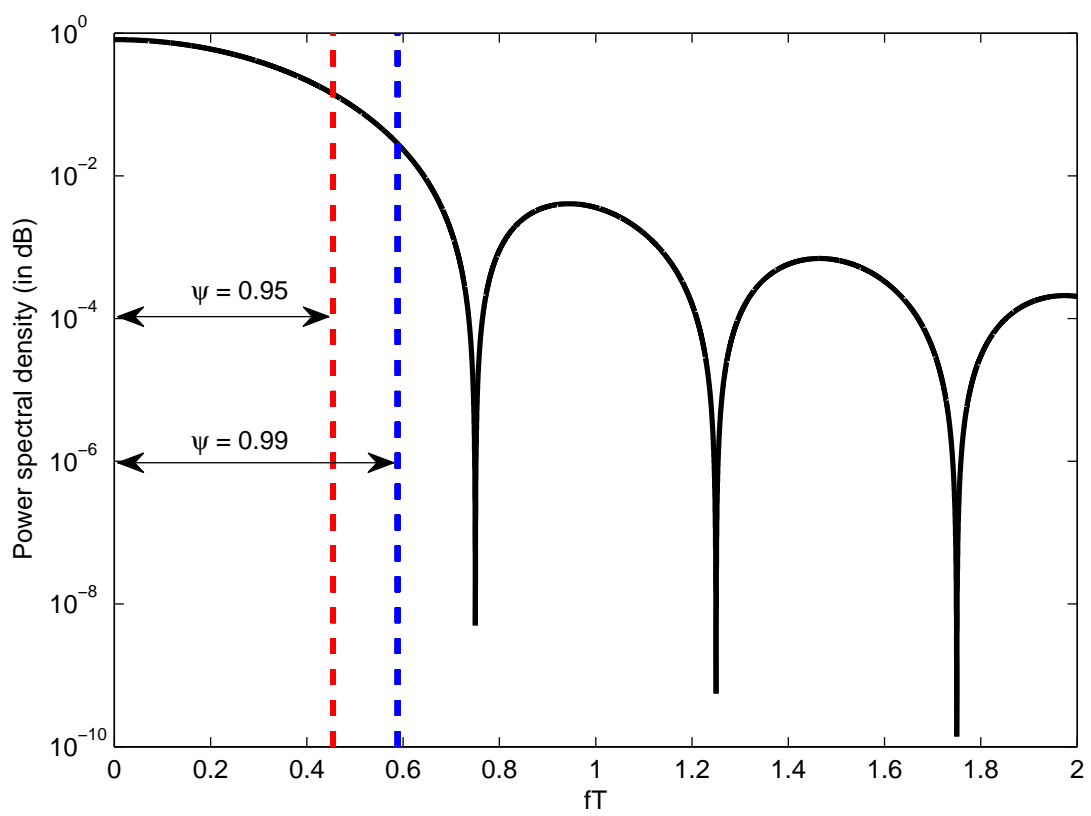

Figure 7.2: Power spectral density of MSK as function of the normalized frequency. Dashed lines delineate starting from the left the 95 and 99-percent power bandwidth.

probability that $X_{i}, i \geq 0$, induces an adjacent-channel collision is

$$
p_{\mathrm{a}}=D\left[\left(\frac{2}{\mathcal{L}}\right)\left(\frac{\mathcal{L}-2}{\mathcal{L}}\right)+\left(\frac{1}{\mathcal{L}}\right)\left(\frac{2}{\mathcal{L}}\right)\right]=\frac{2 D(\mathcal{L}-1)}{\mathcal{L}^{2}} .
$$

Only a certain percentage of a device's transmitted power lies within its selected frequency channel. Let $\psi$ represent the fractional in-band power, which is the fraction of power in the occupied frequency channel. For illustrative purposes, Fig. 7.2 shows the power spectral density of minimum-shift keying (MSK) (binary CPFSK with $h=0.5$ ) as function of the normalized frequency, and furthermore the 95 and 99-percent power bandwidth are delineated. Assume that a fraction $K_{s}=(1-\psi) / 2$, called the adjacent-channel splatter ratio, spills into each of the frequency channels that are adjacent to the one selected by a device. For most practical systems, this is a reasonable assumption, as the fraction of power that spills into frequency channels beyond the adjacent ones is negligible, since they are sufficiently separated in frequency.

Under the model described above, the SINR is provided by $(2.4)$, where the normalized received power is

$$
\Omega_{i, j}= \begin{cases}\psi 10^{\xi_{0, j} / 10} r_{0, j}^{-\alpha} & i=0 \\ \frac{P_{i}}{P_{0}} 10^{\xi_{i, j} / 10} r_{i, j}^{-\alpha} & i \geq 1\end{cases}
$$


and $I_{i}$ is a discrete random variable that may take on three values with the probabilities

$$
I_{i}= \begin{cases}\psi & \text { with probability } p_{\mathrm{c}} \\ K_{s} & \text { with probability } p_{\mathrm{a}} \\ 0 & \text { with probability } p_{\mathrm{n}}\end{cases}
$$

where $p_{\mathrm{n}}=1-p_{\mathrm{c}}-p_{\mathrm{a}}=1-D(3 \mathcal{L}-2) / \mathcal{L}^{2}$ is the probability of no collision.

\subsubsection{Conditional Outage Probability}

Define the variable $Y_{i, j}=I_{i} g_{i, j} \Omega_{i, j}$, where $I_{i}$ is given by $(7.3)$. Taking into account the Nakagami fading and the statistics of $I_{i}$, the $\mathrm{PDF}$ of $Y_{i, j}$ is 119

$$
f_{\mathrm{Y}_{i, j}}(y)=p_{n} \delta(y)+\frac{y^{m_{i}-1}}{\Gamma\left(m_{i, j}\right)}\left[p_{\mathrm{c}}\left(\frac{m_{i, j}}{\psi \Omega_{i, j}}\right)^{m_{i, j}} e^{-\frac{y m_{i, j}}{\psi \Omega_{i, j}}}+p_{\mathrm{a}}\left(\frac{m_{i, j}}{K_{s} \Omega_{i, j}}\right)^{m_{i, j}} e^{-\frac{y m_{i, j}}{K_{s} \Omega_{i, j}}}\right] u(y)
$$

Theorem 7.1.1 The conditioned outage probability is

$$
\epsilon_{j}(\Omega)=1-\exp \left(-\frac{\beta m_{0, j}}{\Omega_{0, j} \mathrm{SNR}}\right) \sum_{\mu=0}^{m_{0, j}-1}\left(\frac{\beta m_{0, j}}{\Omega_{0, j} \mathrm{SNR}}\right)^{\mu} \sum_{t=0}^{\mu} \frac{\mathrm{SNR}^{t}}{(\mu-t) !} \sum_{\substack{\ell_{i} \geq 0 \\ \sum_{i=1}^{M} \ell_{i}=t}} \prod_{i=1}^{M} G_{\ell_{i}}\left(\Omega_{i, j}\right)
$$

where

$$
\begin{aligned}
G_{\ell_{i}}\left(\Omega_{i, j}\right) & =p_{\mathrm{n}} \delta_{\ell_{i}}+\frac{\Gamma\left(\ell_{i}+m_{i}\right)}{\ell_{i} ! \Gamma\left(m_{i, j}\right)}\left[p_{\mathrm{c}} \phi_{i, j}(\psi)+p_{\mathrm{a}} \phi_{i, j}\left(K_{s}\right)\right] \\
\phi_{i, j}(x) & =\left(\frac{x \Omega_{i, j}}{m_{i, j}}\right)^{\ell_{i}}\left(\frac{x \beta m_{0, j} \Omega_{i, j}}{m_{i, j} \Omega_{0, j}}+1\right)^{-\left(m_{i, j}+\ell_{i}\right)} .
\end{aligned}
$$

Proof: The details of the derivation are the same given in Appendix $A$, with the only difference that (7.4) is substitute in (A.9.

Notice that if $\mathrm{ACI}$ is neglected $(\psi=1),(7.5)$ reduces to 2.9 .

\subsubsection{Spatially Averaged Outage Probability}

Assume that a fixed number $M$ of interfering devices are independently and uniformly distributed over the region of area $|\mathcal{A}|$; i.e., that the interfering devices are drawn from a $\overline{\mathrm{BPP}}$ of intensity $\lambda=M /|\mathcal{A}|$. Furthermore, consider an annular region with inner radius $r_{\text {in }}$ and outer radius $r_{\text {out }}$. 


\section{Unshadowed Scenario}

Following the same steps as in Sec. 2.3.1 and starting from (7.5), the spatially averaged outage probability for a $\mathrm{BPP}$ can be expressed as

$$
\epsilon_{j}[\lambda]=1-\exp \left(-\frac{\beta m_{0, j}}{\Omega_{0, j} \mathrm{SNR}}\right) \sum_{\mu=0}^{m_{0, j}-1}\left(\frac{\beta m_{0, j}}{\Omega_{0, j} \mathrm{SNR}}\right)^{\mu} \sum_{t=0}^{\mu} \frac{\mathrm{SNR}^{t}}{(\mu-t) !} \sum_{\ell_{i} \geq 0} \prod_{i=1}^{M} \mathbb{E}_{\boldsymbol{\Omega}}\left\{G_{\ell_{i}}\left(\Omega_{i, j}\right)\right\}
$$

where

$$
\begin{aligned}
\mathbb{E}_{\boldsymbol{\Omega}}\left\{G_{\ell_{i}}\left(\Omega_{i, j}\right)\right\}= & p_{\mathrm{n}} \delta_{\ell_{i}}+\frac{2 m_{i, j}^{m_{i, j}} \Gamma\left(\ell_{i}+m_{i, j}\right)}{\alpha\left(\ell_{i} !\right) c_{i}^{\frac{2}{\alpha}}\left(r_{\text {out }}^{2}-r_{\text {in }}^{2}\right) \Gamma\left(m_{i, j}\right)\left(\beta m_{0, j} / \Omega_{0, j}\right)^{\left(m_{i, j}+\ell_{i}\right)}} \\
& \left\{p_{\mathrm{c}}\left[\mathcal{I}\left(\frac{\psi c_{i}}{r_{\text {out }}^{\alpha}}\right)-\mathcal{I}\left(\frac{\psi c_{i}}{r_{\text {in }}^{\alpha}}\right)\right]+p_{\mathrm{a}}\left[\mathcal{I}\left(\frac{K_{s} c_{i}}{r_{\text {out }}^{\alpha}}\right)-\mathcal{I}\left(\frac{K_{s} c_{i}}{r_{\text {in }}^{\alpha}}\right)\right]\right\} \\
\mathcal{I}(x)= & \frac{{ }_{2} F_{1}\left(\left[m_{i, j}+\ell_{i}, m_{i, j}+\frac{2}{\alpha}\right] ; m_{i, j}+\frac{2}{\alpha}+1 ;-\frac{m_{i, j} \Omega_{0, j}}{x \beta m_{0, j}}\right)}{x^{m_{i, j}+\frac{2}{\alpha}}\left(m_{i, j}+\frac{2}{\alpha}\right)}
\end{aligned}
$$

\section{Log-Normal Shadowing}

In the presence of log-normal shadowing the coefficient $\left\{\xi_{i, j}\right\}$ are independent and identically distributed zero-mean Gaussian with standard deviation $\sigma_{\mathrm{s}} \mathrm{dB}$. The spatially average outage probability can be obtained from $(7.8$ by following the same steps as in Sec. 2.3.1. In particular by bringing all the terms that are function of $\Omega$ inside the product, and averaging over it, it follows that

$$
\epsilon_{j}[\lambda]=1-\sum_{\mu=0}^{m_{0, j}-1} \sum_{t=0}^{\mu} \frac{\mathrm{SNR}^{t}}{(\mu-t) !} \sum_{\substack{\ell_{i} \geq 0 \\ \sum_{i=1}^{M} \ell_{i}=t}} \prod_{i=1}^{M} \mathbb{E}_{\boldsymbol{\Omega}}\left\{\exp \left(-\frac{\beta m_{0, j}}{\Omega_{0, j} \mathrm{SNR}}\right)\left(\frac{\beta m_{0, j}}{\Omega_{0, j} \mathrm{SNR}}\right)^{\mu} G_{\ell_{i}}\left(\Omega_{i, j}\right)\right\}
$$

When the distance between the reference receiver and the source transmitter $r_{0, j}$ is fixed, but shadowing is present, $\Omega_{0, j}$ becomes a log-normal random variable with $\mathrm{PDF}$ given by 2.37), while $\Omega_{i, j}, i=1, \ldots, M$, has $\mathrm{PDF}$ given by (2.38). Making these substitutions yields

$$
\begin{aligned}
\epsilon_{j}[\lambda]= & \sum_{s=0}^{m_{0, j}-1} \sum_{t=0}^{s} \frac{\mathrm{SNR}^{t}}{(s-t) !} \sum_{\ell_{i} \geq 0}\left\{\int_{0}^{\infty} \exp \left\{-\frac{\beta m_{0, j}}{\psi y \mathrm{SNR}}\right\}\left(\frac{\beta m_{0, j}}{\psi y \mathrm{SNR}}\right)^{s}\right. \\
& \left.\times \prod_{i=1}^{M}\left[p_{n} \delta_{\ell_{i}}+p_{c} \phi_{i, j}(y, \psi)+p_{a} \phi_{i, j}\left(y, K_{s}\right)\right] f_{\Omega_{0, j}}(y) d y\right\}
\end{aligned}
$$


where

$$
\phi_{i, j}(y, \chi)=\frac{\Gamma\left(\ell_{i}+m_{i, j}\right)}{\ell_{i} ! \Gamma\left(m_{i, j}\right)} \int_{0}^{\infty} f_{\Omega_{i, j}}(\omega)\left(\frac{\chi \omega}{m_{i, j}}\right)^{\ell_{i}}\left(\frac{\chi \beta m_{0, j} \omega}{\psi m_{i, j} y}+1\right)^{-\left(m_{i, j}+\ell_{i}\right)} d \omega .
$$

The integral in $(7.13)$ can be evaluated numerically by Simpson's method, which provides a good tradeoff between accuracy and speed, while the integral in $(7.12)$ can be evaluated through Monte Carlo simulation.

\subsection{Modulation-Constrained Area Spectral Efficiency}

When written as a function of $\lambda$, the spatially averaged outage probability $\epsilon_{j}(\lambda)$ is often constrained to not exceed a maximum value $\hat{\epsilon}_{\text {channel }} \in[0,1]$; i.e., $\epsilon(\lambda) \leq \hat{\epsilon}_{\text {channel }}$. Under such a constraint on the channel outage, the maximum density of transmissions is quantified by the ASE, which is

$$
\mathcal{S}_{\text {ase }}(\zeta)=\epsilon^{-1}\left(\hat{\epsilon}_{\text {channel }}\right)\left(1-\hat{\epsilon}_{\text {channel }}\right) \mathrm{T}_{\text {raw }}
$$

where $\mathrm{T}_{\text {raw }}$ is the raw per-link throughput in the absence of an outage, $\epsilon^{-1}\left(\hat{\epsilon}_{\text {channel }}\right)$ is the density of the underlying process whose spatially averaged outage probability satisfies the constraint $\epsilon(\lambda) \leq \hat{\epsilon}_{\text {channel }}$, with equality ${ }^{1}$, and $\left(1-\hat{\epsilon}_{\text {channel }}\right)$ ensures that only successful transmissions are counted.

The ASE embodies the tradeoff between the outage probability and spatial density. In (7.14), the tradeoff is quantified by fixing $\epsilon$ and varying $\lambda$. An alternative representation, which is more applicable to the approach taken in this chapter, is to fix $\lambda$ and allow $\epsilon$ to vary. This allows the ASE to be expressed as a function of $\lambda$,

$$
\mathcal{S}_{\text {ase }}(\lambda)=\lambda[1-\epsilon[\lambda]] \mathrm{T}_{\text {raw }} .
$$

As originally defined in [75], the ASE is a function of the SINR threshold $\beta$ and is found without making any assumptions about the type of modulation or channel code. In practice, $\beta$ is a function of the modulation and coding that is used. Let $C(\gamma)$ denote the maximum achievable rate, in bps, that can be supported by the chosen modulation at an

\footnotetext{
${ }^{1}$ Since $\epsilon$ is a monotonically increasing function of $\lambda$, the ASE is maximized when the constraint $\epsilon \leq \hat{\epsilon}_{\text {channel }}$ is met with equality.
} 


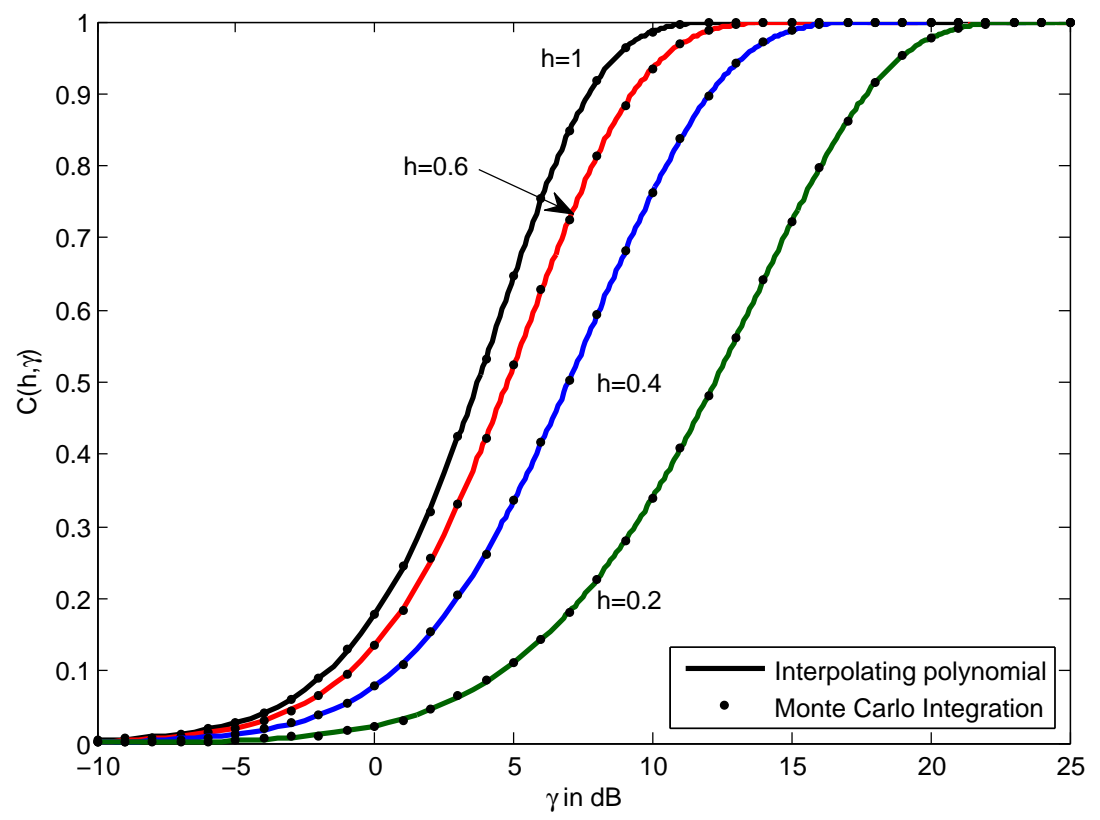

Figure 7.3: The symmetric-information rate $C(h, \gamma)$ of noncoherent binary CPFSK with modulation indices $h=[0.2,0.4,0.6,1]$. The markers were found through Monte Carlo integration while the curves are a polynomial fit.

instantaneous SINR of $\gamma$ assuming equally likely input symbols; i.e., it is the modulationconstrained capacity or symmetric-information rate. If a capacity-achieving rate- $R$ code is used, then an outage will occur when $C(\gamma) \leq R$. Since $C(\gamma)$ is monotonic, it follows that $\beta$ is the value for which $C(\beta)=R$, and therefore it can be written that $\beta=C^{-1}(R)$. If it is assumed that each codeword is transmitted using one hop, then the outage probability with SINR threshold $\beta=C^{-1}(R)$ provides an accurate prediction of the codeword error rate [74.

[FH] systems often use noncoherent CPFSK [74, 120]. As already mentioned, a key parameter in CPFSK systems is the modulation index $h$, which represents the relative spacing between tones. To emphasize the dependence of the capacity on $h, C(h, \gamma)$ denotes the symmetric-information rate of CPFSK with modulation index $h$. The symmetric-information rate of noncoherent CPFSK is given in [120] for various $h$, and is found by computing the average mutual information between the channel input and the output of the noncoherent AWGN channel. In a noncoherent system, the mutual integration integral cannot be solved in closed form and is most effectively found using Monte Carlo integration. Fig. 7.3, which is adapted from Fig. 1 of [120], shows the symmetric information rate of binary CPFSK as a function of $\gamma$ for various $h$. In the figure, Monte Carlo integration is used to obtain the 


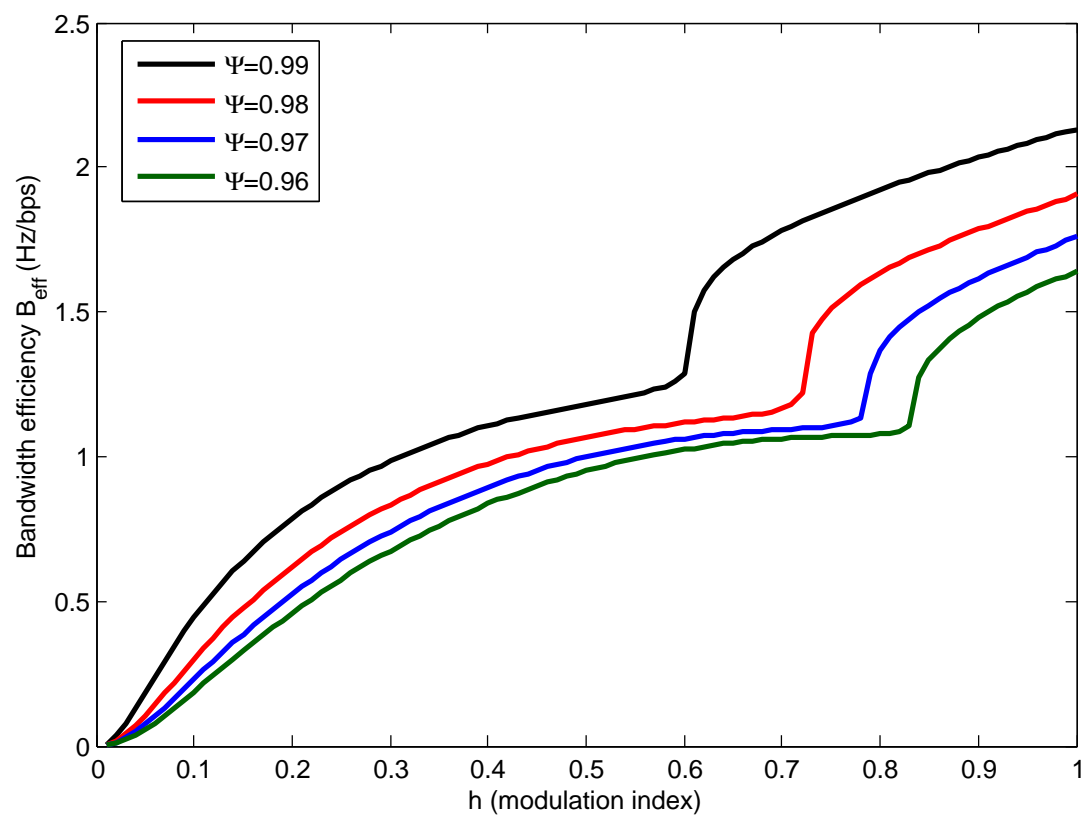

Figure 7.4: Bandwidth efficiency $B_{\text {eff }}$ as function of the modulation index $h$ for four values of the fractional in-band power $\psi=[0.96,0.97,0.98,0.99]$.

symmetric information rate at discrete values of $\gamma$, and a polynomial fit is used to determine the other values. For any value of $h$, the value of the SINR threshold $\beta$ can be found from the corresponding curve by finding the value of $\gamma$ for which $C(h, \gamma)=R$. For instance, when $R=1 / 2$ and $h=1$, the required $\beta=3.7 \mathrm{~dB}$. In [121], it was found that in practice, and over a wide range of code rates, turbo-coded noncoherent CPFSK is consistently about $1 \mathrm{~dB}$ away from the corresponding modulation-constrained capacity limit. Thus, the $\beta$ required in practice will generally be higher than the value obtained from the capacity limit by a small margin. For instance, if the actual system requires a $1 \mathrm{~dB}$ margin over the capacity limit, then the SINR threshold for noncoherent binary CPFSK with $R=1 / 2$ and $h=1$ should be set to $\beta=4.7 \mathrm{~dB}$.

When accounting for modulation and coding, the maximum data transmission rate is determined by the bandwidth $B / \mathcal{L}$ of a frequency channel, the fractional in-band power $\psi$, the spectral efficiency of the modulation, and the code rate. Let $\eta$ be the spectral efficiency of the modulation, given in symbols per second per $\mathrm{Hz}$, and defined by the symbol rate divided by the $100 \psi \%$-power bandwidth of the modulation. Since many symbols per hop are assumed, the spectral efficiency of CPFSK can be found by numerically integrating (3.4-61) 
of [122] and then inverting the result. Fig. 7.4 shows the bandwidth efficiency $B_{\text {eff }}(\mathrm{Hz} / \mathrm{bps})$, which is the inverse of the spectral efficiency, as function of the modulation index $h$ for four different values of the fractional in-band power $\psi$. To emphasize the dependence of $\eta$ on $h$ and $\psi$, the spectral efficiency of CPFSK is denoted as $\eta(h, \psi)$. When combined with a rate- $R$ code, the spectral efficiency becomes $R \eta(h, \psi)$ bps per Hertz (bps/Hz), where $R$ is the ratio of information bits to code symbols. Since the signal occupies a frequency channel with $100 \psi \%$-power bandwidth $B / \mathcal{L} \mathrm{Hz}$, the maximum data rate in bps supported by a single link operating with a duty factor $D$ is

$$
\mathrm{T}_{\text {raw }}=\frac{R D \eta(h, \psi) B}{\mathcal{L}} .
$$

Substituting (7.16) into 7.15 and dividing by the system bandwidth $B$ gives the normalized and spatially averaged MASE,

$$
\mathcal{S}_{\text {ase }}^{\prime}(\lambda)=\frac{\lambda R D \eta(h, \psi)[1-\epsilon(\lambda)]}{\mathcal{L}}
$$

which assumes units of bps/Hz per unit area. In contrast with (7.15), this form of ASE explicitly takes into account the code rate $R$, the spectral efficiency of the modulation $\eta(h, \psi)$, and the number of frequency channels.

\subsection{Optimization and Rate Allocation Policies}

In this section two different rate allocation policies are considered. First, Sec. 7.3.1 assumes that a common rate is chosen and an optimization methodology is described to jointly and efficiently find the rate, modulation characteristics, and number of hopping frequencies that maximize the throughput of the system. The description of the optimization method is followed by several examples that highlights the effectiveness of the optimization. Sec. 7.3.2 assumes that the network is able to perform rate adaptation [123 based on the quality of the channel in order to meet a specific constraint on the channel outage. Similar to the previous subsection, an optimization methodology is provided that finds the modulation characteristics that maximize the MASE and this methodology is applied to several cases to emphasize the benefit of the optimization. 


\subsubsection{Fixed Rate Optimization}

Let $\boldsymbol{\theta}=\{\mathcal{L}, R, h, \psi\}$ and $C_{\text {cost }}(\boldsymbol{\theta})$ represent a cost function. The goal of the network optimization is to determine the $\boldsymbol{\theta}$ that minimizes an appropriate cost function. Fix the value of $\lambda$, and let $\mathcal{S}_{\text {ase }}^{\prime}(\boldsymbol{\theta})$ represent the normalized MASE at that $\lambda$ as a function of $\boldsymbol{\theta}$. Furthermore, $C_{\text {cost }}(\boldsymbol{\theta})=-\mathcal{S}_{\text {ase }}^{\prime}(\boldsymbol{\theta})$ is chosen as cost function so that the optimization maximizes the normalized MASE, For instance,

$$
\mathcal{S}_{\mathrm{opt}}^{\prime}=\max _{\boldsymbol{\theta}} \mathcal{S}_{\mathrm{ase}}^{\prime}(\boldsymbol{\theta})
$$

\section{Downhill Simplex Optimization}

After an initial study of the optimization surface [47, 124], which has been done by performing an exhaustive evaluation of $\mathcal{S}_{\text {ase }}^{\prime}(\boldsymbol{\theta})$ over a large set of discretized $\boldsymbol{\theta}$, it is found that it is convex. Because of this property of the optimization problem, a more efficient search technique can be used. However, since $\mathcal{S}_{\text {ase }}^{\prime}(\boldsymbol{\theta})$ is a complicated nonlinear function of $\boldsymbol{\theta}$, it is difficult to obtain analytical or numerical derivatives. For this reason, a direct search is preferred over a gradient search. In particular, the Nelder-Mead method of downhill simplex optimization 125 is an appropriate and efficient solution for this optimization problem.

In four dimensions, the Nelder-Mead method works by evaluating the cost function $C(\boldsymbol{\theta})$ at the five corners of a pentachoron; i.e., a 4-dimensional convex regular polytope or hyperpyramid. After each iteration, one corner of the pentachoron is moved until it contains the minimum, at which point the pentachoron is made smaller. In our implementation, the first corner is initially at $\{\mathcal{L}, R, h, \psi\}=\{20,0.5,0.5,0.975\}$ and the other corners are at distances 1, 0.025, 0.025, and 0.005 from the first corner along each of the four dimensions. Although $\mathcal{L}$ needs to be an integer, during the optimization we allow it to be real valued to ensure a continuous optimization surface.

Let $\boldsymbol{\theta}_{1}, \ldots, \boldsymbol{\theta}_{5}$ represent the corners of the pentachoron sorted in ascending cost; i.e., $C\left(\boldsymbol{\theta}_{1}\right) \leq C\left(\boldsymbol{\theta}_{2}\right) \leq \ldots \leq C\left(\boldsymbol{\theta}_{5}\right)$. An iteration proceeds by first reflecting $\boldsymbol{\theta}_{5}$ across the opposing face of the pentachoron to produce a candidate corner $\boldsymbol{\theta}_{\mathrm{r}}$ whose cost is computed. If $C\left(\boldsymbol{\theta}_{1}\right) \leq C\left(\boldsymbol{\theta}_{\mathbf{r}}\right)<C\left(\boldsymbol{\theta}_{4}\right)$, then corner $\boldsymbol{\theta}_{5}$ is replaced by $\boldsymbol{\theta}_{\mathbf{r}}$. The points are re-sorted and the algorithm moves on to the next iteration. If $C\left(\boldsymbol{\theta}_{\mathbf{r}}\right)<C\left(\boldsymbol{\theta}_{1}\right)$, then an expanded pentachoron 
is considered by doubling the distance from $\boldsymbol{\theta}_{\mathrm{r}}$ to the face defined by the other four corners, thereby producing another candidate corner $\boldsymbol{\theta}_{\mathrm{s}}$ whose cost is computed. If $C\left(\boldsymbol{\theta}_{\mathrm{s}}\right)<C\left(\boldsymbol{\theta}_{\mathrm{r}}\right)$, then the expanded pentachoron is accepted (by replacing $\boldsymbol{\theta}_{5}$ with $\boldsymbol{\theta}_{\mathbf{s}}$ ), otherwise the reflected (but unexpanded) pentachoron is accepted by replacing $\boldsymbol{\theta}_{5}$ with $\boldsymbol{\theta}_{\mathbf{r}}$. If $C\left(\boldsymbol{\theta}_{\mathbf{r}}\right) \geq C\left(\boldsymbol{\theta}_{4}\right)$ then a contraction is performed by halving the distance between the better of $\boldsymbol{\theta}_{5}$ and $\boldsymbol{\theta}_{\mathrm{r}}$ and the face defined by the other four corners. The contracted pentachoron is accepted if this new corner has a lower cost than the one it displaced. Otherwise, if none of the above conditions is satisfied then the minimum must lie inside the pentachoron, so it is shrunk by halving the length of each edge while maintaining the same centroid.

\section{Optimization Results}

By using the Nelder-Mead method and the closed-form expressions provided in Sec. 7.1.3, optimization results are obtained for a range of densities $\lambda$ with interfering devices drawn from a BPP. Unless otherwise stated, the remainder of this chapter considers a network with $M=50$ interfering devices. Assume the source is located at unit distance from the reference receiver. Assume the transmitter uses binary CPFSK. The duty factor is fixed to $D=1$, the path loss exponent is $\alpha=3$, the $\mathrm{SNR}$ is $\mathrm{SNR}=10 \mathrm{~dB}$, and the exclusion zone has radius $r_{\text {in }}=0.25$. Three fading models are considered: Rayleigh fading $\left(m_{i, j}=1\right.$ for all $i)$, Nakagami fading $\left(m_{i, j}=4\right.$ for all $\left.i\right)$, and mixed fading $\left(m_{0, j}=4\right.$ and $m_{i, j}=1$ for $\left.i \geq 1\right)$. Both unshadowed $\left(\sigma_{\mathrm{s}}=0 \mathrm{~dB}\right)$ and shadowed $\left(\sigma_{\mathrm{s}}=8 \mathrm{~dB}\right)$ environments are considered. Two values of $r_{\text {out }}$ are considered: $r_{\text {out }}=2$, and $r_{\text {out }}=4$, corresponding to a moderately dense $(\lambda \approx 4)$ and sparse $(\lambda \approx 1)$ network, respectively.

The results of the optimization are summarized in Table 7.1 when ACI is neglected. For each set of parameters, the $\{\mathcal{L}, R, h\}$ that maximize the MASE are listed, along with the corresponding normalized MASE $\mathcal{S}_{\text {opt }}^{\prime}$.

When ACI is neglected, the optimal modulation index is $h=0.59$ for all network configurations, which coincides with the optimal value found in [120 for Rayleigh fading. The optimal $\mathcal{L}$ is larger for denser networks and when the signals become more $\mathrm{LOS}\left(m_{i, j}>1\right)$. The denser network requires a higher $R$ than the sparser network. At first inspection, the last result may seem counter-intuitive. One would ordinarily expect a denser network to 
Table 7.1: Optimal parameter values when ACI due to spectral splatter is ignored, and the corresponding MASE in units of bps $/ k H z-m^{2}$.

\begin{tabular}{|c|c|c|c|c|c|c|c|c|c|}
\hline$r_{\text {out }}$ & $\sigma_{s}^{2}$ & $m_{0, j}$ & $m_{i, j}$ & $\mathcal{L}$ & $R$ & $h$ & $\mathcal{S}_{\mathrm{opt}}^{\prime}$ & $\mathcal{S}_{\text {sub }}^{\prime}$ & $\mathcal{S}_{1}^{\prime}$ \\
\hline \multirow[t]{6}{*}{2} & \multirow[t]{3}{*}{0} & 1 & 1 & 31 & 0.61 & 0.59 & 15.92 & 3.31 & 13.37 \\
\hline & & 4 & 4 & 42 & 0.66 & 0.59 & 17.09 & 4.05 & 14.43 \\
\hline & & 4 & 1 & 36 & 0.65 & 0.59 & 19.82 & 4.13 & 16.69 \\
\hline & \multirow[t]{3}{*}{8} & 1 & 1 & 30 & 0.63 & 0.59 & 16.65 & 3.30 & 14.07 \\
\hline & & 4 & 4 & 38 & 0.65 & 0.59 & 17.63 & 4.00 & 14.61 \\
\hline & & 4 & 1 & 33 & 0.64 & 0.59 & 20.29 & 4.08 & 17.10 \\
\hline \multirow[t]{6}{*}{4} & \multirow[t]{3}{*}{0} & 1 & 1 & 12 & 0.54 & 0.59 & 9.73 & 0.89 & 7.86 \\
\hline & & 4 & 4 & 15 & 0.50 & 0.59 & 10.65 & 1.12 & 8.46 \\
\hline & & 4 & 1 & 14 & 0.51 & 0.59 & 11.85 & 1.12 & 9.49 \\
\hline & \multirow[t]{3}{*}{8} & 1 & 1 & 11 & 0.54 & 0.59 & 10.08 & 0.89 & 8.22 \\
\hline & & 4 & 4 & 14 & 0.52 & 0.59 & 10.77 & 1.10 & 8.64 \\
\hline & & 4 & 1 & 13 & 0.53 & 0.59 & 12.01 & 1.10 & 9.68 \\
\hline
\end{tabular}

require the error-protection of a lower-rate code. However, the sparser network uses a lower value of $\mathcal{L}$, and hence, has a higher probability of collision $p_{i}$. The lower code rate used by the sparse network helps to offset this higher collision probability.

From Table 7.1, the influence of the fading distribution can be observed. Performance is worst under the assumption that all channels undergo Rayleigh fading, but this is a pessimistic assumption. Performance improves when all channels have a common $m_{i, j}=4$, but it is unrealistic to assume that all interferers are in the LOS, Performance is further improved when the interferers undergo Rayleigh fading while the source undergoes better than Rayleigh fading $\left(m_{0, j}=4\right)$. Shadowing does not significantly influence performance, and actually slightly improves the MASE while increasing the outage probability.

In addition to showing the performance when the optimal parameter values are used, Table 7.1 shows the performance when a typical set $\{\mathcal{L}, R, h\}=\{200,0.5,1\}$ of suboptimal parameters is used. For this $R$ and $h$, the SINR threshold is $\beta=3.7 \mathrm{~dB}$. The table lists the normalized MASE $\mathcal{S}_{\text {sub }}^{\prime}$ that results when this suboptimal set of parameters is used. By comparing the values of $\mathcal{S}_{\text {opt }}^{\prime}$ and $\mathcal{S}_{\text {sub }}^{\prime}$, it can be seen that using the optimized values 
Table 7.2: Optimal parameter values when ACI due to spectral splatter is considered, and the corresponding MASE in units of $b p s / k H z-m^{2}$.

\begin{tabular}{|c|c|c|c|c|c|c|c|c|c|}
\hline$r_{\text {out }}$ & $\sigma_{s}^{2}$ & $m_{0,}$ & $m_{i, j}$ & $\mathcal{L}$ & $R$ & $h$ & $\psi$ & $\mathcal{S}_{\mathrm{opt}}^{\prime}$ & $\mathcal{S}_{1}^{\prime}$ \\
\hline \multirow[t]{6}{*}{2} & \multirow[t]{3}{*}{0} & 1 & 1 & 36 & 0.64 & 0.81 & 0.96 & 17.59 & 14.62 \\
\hline & & 4 & 4 & 46 & 0.64 & 0.81 & 0.96 & 18.88 & 15.74 \\
\hline & & 4 & 1 & 41 & 0.64 & 0.81 & 0.96 & 21.68 & 18.09 \\
\hline & \multirow[t]{3}{*}{8} & 1 & 1 & 34 & 0.64 & 0.81 & 0.96 & 18.29 & 15.31 \\
\hline & & 4 & 4 & 44 & 0.66 & 0.81 & 0.96 & 19.36 & 16.14 \\
\hline & & 4 & 1 & 38 & 0.64 & 0.81 & 0.96 & 22.09 & 18.46 \\
\hline \multirow[t]{6}{*}{4} & \multirow[t]{3}{*}{0} & 1 & 1 & 12 & 0.54 & 0.85 & 0.95 & 11.87 & 9.56 \\
\hline & & 4 & 4 & 16 & 0.54 & 0.85 & 0.95 & 13.05 & 10.29 \\
\hline & & 4 & 1 & 15 & 0.54 & 0.85 & 0.95 & 14.47 & 11.55 \\
\hline & \multirow[t]{3}{*}{8} & 1 & 1 & 12 & 0.58 & 0.85 & 0.95 & 12.22 & 9.95 \\
\hline & & 4 & 4 & 15 & 0.54 & 0.85 & 0.95 & 13.13 & 10.52 \\
\hline & & 4 & 1 & 14 & 0.57 & 0.85 & 0.95 & 14.55 & 11.66 \\
\hline
\end{tabular}

increases the MASE relative to the suboptimal system by a factor of approximately 5 and 10 in the dense and sparse networks, respectively.

The value of $\mathcal{S}_{\text {opt }}^{\prime}$ given in Table 7.1 assumes that $\{R, h, \beta\}$ are related by the modulationconstrained AWGN capacity. This assumption requires that the interference be Gaussian and that infinitely-long capacity-achieving codes be used. However, actual systems require a threshold that is higher than this ideal value by some margin. The column labeled $\mathcal{S}_{1}^{\prime}$ gives the normalized MASE when a 1-dB margin is used with the listed values of $\{\mathcal{L}, R, h\}$. A modest loss in MASE is observed when this margin is used.

In Table 7.2, the results of the optimization are summarized. The optimal fractional inband powers are $\psi=0.96$ for the a dense network and $\psi=0.95$ for a sparse network. The optimal modulation indices $h$ are larger than the ones found when ACI was not considered, while the code rates are almost the same. The optimal numbers of channels $\mathcal{L}$ are the same or slightly larger than the ones found when ACI was neglected. As before, the margin of 1 $\mathrm{dB}$ added to compensate the non-ideality of the channel degrades performance by a modest amount. 


\subsubsection{Rate Adaptation}

Due to the fact that interferers move, join, or leave the network, the outage probability can vary very substantially from time to time, and it might assume values that are not supported in some of the modern applications. For this reason, the rate is often adapted (e.g., [126, 127]) by assigning a different code rate based on the quality of the channel to increase the system capacity and/or to satisfy a specific outage constraint. While in the previous subsection, an optimal common rate is found together with the modulation characteristics in order to maximize the throughput of a $\mathrm{FH}$ system, here the effect of link adaptation is taken into account by assuming that a mobile is able to estimate the channel and appropriately adapt its rate in order to meet a given outage constraint. The adaptation assumes that the distribution of the outage probability is known; i.e., that the transmitter has some way to estimate 7.5 for its intended receiver. This function can be precisely estimated if the transmitter locations, transmitter powers, shadowing coefficients, and the parameters in (7.3), i.e. $\psi, K_{s}, p_{\mathrm{c}}$ and $p_{\mathrm{a}}$, are known. Importantly, the instantaneous fading gains or the particular channel selected by the transmitter need not be known. In the absence of these parameters, a simple adaptive scheme could request a higher rate when the observed outage probability (as determined by a CRC check) is below the target and requests a lower rate when it is above. While the rate is adapted, the number of frequency-hopping channels, the modulation index, and the fractional in-band power are jointly optimized.

\section{Optimization Algorithm}

Under an outage constraint, and averaging over the distribution of the rate when it is adapted to meet a specific outage contraint, the MASE can be written as follows

$$
\mathcal{S}_{\text {ase }}^{\prime}(\lambda)=\frac{\lambda \eta(h, \psi) D\left(1-\hat{\epsilon}_{\text {channel }}\right) \mathbb{E}_{\boldsymbol{\Omega}}[R]}{\mathcal{L}}
$$

which can be interpreted as the spatial density of transmission that achieves a target conditional outage probability, where the conditioning is with respect to the network topology.

For a given modulation index $h$, a given number of frequency channels, and a given fractional in-band power, the average code rate $\mathbb{E}_{\boldsymbol{\Omega}}[R]$ can be found using a Monte Carlo approach as follows. Draw a realization of the network by placing $M$ devices within an area 
$|\mathcal{A}|$ according to a specific spatial distribution. Compute the path loss from each device to each reference receiver, applying randomly generated shadowing factors. By setting the outage equal to the outage constraint $\hat{\epsilon}_{\text {channel }}$, invert the outage probability expression given by $(7.5)$ to determine the adequate SINR threshold $\beta$. Next, find the rate $R_{i}=C(h, \beta)$ that corresponds to the SINR threshold for the $i^{t h}$ topology and store its value. Repeat this process for a large number of networks $\Upsilon$. Compute the average code rate as follows

$$
\mathbb{E}_{\boldsymbol{\Omega}}[R]=\frac{1}{\Upsilon} \sum_{i=1}^{\Upsilon} R_{i} .
$$

In this case, the optimization can be accomplished using an exhaustive search by performing the following steps:

1. Pick a value of $h$.

2. Pick a value of $\Psi$.

3. Pick a value of $\mathcal{L}$.

4. Compute the average code rate $\mathbb{E}_{\boldsymbol{\Omega}}[R]$, as described above, and find the spectral efficiency $\eta(h, \Psi)$ corresponding to the current $h$ and $\Psi$.

5. Use (7.19) to compute the normalized MASE,

6. Return to step 4 until all $\mathcal{L}$ are considered.

7. Return to step 3 until all $\Psi$ are considered.

8. Return to step 2 until all $h$ are considered.

The above procedure will find the $\mathcal{S}_{\text {ase }}^{\prime}(\lambda)$ for each $\{\mathcal{L}, h, \Psi\}$ considered, and the optimal value of these parameters are the ones that maximize $\mathcal{S}_{\text {ase }}^{\prime}(\lambda)$. For instance,

$$
\mathcal{S}_{\text {opt }}^{\prime}=\max _{\mathcal{L}, h, \Psi} \mathcal{S}_{\text {ase }}^{\prime}\left(\boldsymbol{\theta} \mid \mathbb{E}_{\boldsymbol{\Omega}}[R]\right)
$$

By limiting $\mathcal{L}$ to be integer valued, the number of values is finite and an exhaustive search up to some maximum value is feasible. For the exhaustive search results presented in this subsection, $h$ was quantized to a spacing of 0.01 over the range $0 \leq h \leq 1$ and $\Psi$ was quantized to a spacing of 0.005 over the range $0.90 \leq \Psi \leq 0.99$. 


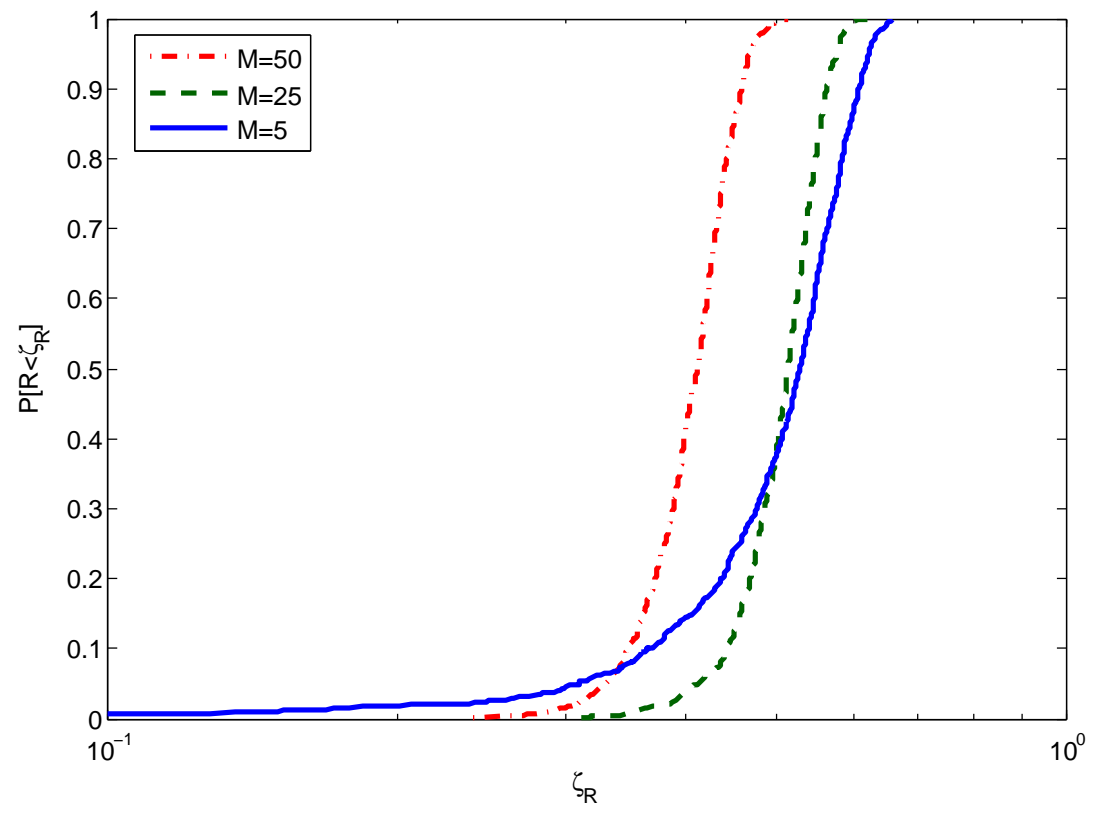

Figure 7.5: CDF of the code rate when the network is optimized. Three curves are provided: a) dense network $(M=50)$; b) moderate dense network $(M=25)$; c) sparse network $(M=5)$.

\section{Optimization Results}

The optimization is done by considering $\Upsilon=10,000$ different network topologies. Binary CPFSK is used, and the rate is adapted in order to satisfy a typical outage constraint of $\hat{\epsilon}_{\text {channel }}=0.1$.

Fig. 7.5 illustrates the variability of the code rate through its $\mathrm{CDF}$ in the presence of mixed fading and shadowing. The figure shows three curves, corresponding to the case of a dense network $(M=50)$, a moderately dense network $(M=25)$ and a sparse network $(M=5)$. The outer radius of the annular network is fixed to $r_{\text {out }}=2$. The network parameters are independently optimized for each network density. Fig. 7.5 shows that the $\mathrm{CDF}$ of the code rate is quite steep for all three scenarios, which emphasizes the fairness in the rate selection. However, the curves are steeper for dense networks than for sparse networks, since in a sparse network there are fewer interferers and therefore more variability in the SINR and code rate from one realization to the next.

The results of the optimization are shown in Table 7.3 . For each set of parameters, the $\{\mathcal{L}, h, \Psi\}$ that maximize the normalized MASE are listed, along with the corresponding 
Table 7.3: Optimal parameter values when ACI due to spectral splatter is considered, and the corresponding MASE in units of bps/ $\mathrm{kHz}-\mathrm{m}^{2}$

\begin{tabular}{|c|c|c|c|c|c|c|c|c|c|}
\hline$r_{\text {out }}$ & $\sigma_{s}^{2}$ & $m_{0, j}$ & $m_{i, j}$ & $\mathcal{L}$ & $\mathbb{E}_{\boldsymbol{\Omega}}[R]$ & $h$ & $\psi$ & $\mathcal{S}_{\mathrm{opt}}^{\prime}$ & $\mathcal{S}_{\text {sub }}^{\prime}$ \\
\hline \multirow[t]{6}{*}{2} & \multirow[t]{3}{*}{0} & 1 & 1 & 315 & 0.07 & 0.80 & 0.96 & 0.79 & 0.50 \\
\hline & & 4 & 4 & 280 & 0.36 & 0.80 & 0.96 & 4.42 & 2.99 \\
\hline & & 4 & 1 & 279 & 0.41 & 0.80 & 0.96 & 5.00 & 3.56 \\
\hline & \multirow[t]{3}{*}{8} & 1 & 1 & 320 & 0.07 & 0.80 & 0.96 & 0.76 & 0.40 \\
\hline & & 4 & 4 & 290 & 0.42 & 0.80 & 0.96 & 4.24 & 2.27 \\
\hline & & 4 & 1 & 290 & 0.38 & 0.80 & 0.96 & 4.70 & 3.02 \\
\hline \multirow[t]{6}{*}{4} & \multirow[t]{3}{*}{0} & 1 & 1 & 73 & 0.05 & 0.84 & 0.95 & 0.63 & 0.32 \\
\hline & & 4 & 4 & 80 & 0.35 & 0.84 & 0.95 & 3.74 & 1.87 \\
\hline & & 4 & 1 & 75 & 0.36 & 0.84 & 0.95 & 4.09 & 1.91 \\
\hline & \multirow[t]{3}{*}{8} & 1 & 1 & 95 & 0.05 & 0.84 & 0.95 & 0.49 & 0.28 \\
\hline & & 4 & 4 & 130 & 0.40 & 0.84 & 0.95 & 2.65 & 1.75 \\
\hline & & 4 & 1 & 100 & 0.35 & 0.84 & 0.95 & 3.03 & 1.80 \\
\hline
\end{tabular}

normalized MASE $\mathcal{S}_{\text {opt }}^{\prime}$. In addition to showing the normalized MASE when using the optimal parameters, the normalized MASE $\mathcal{S}_{\text {sub }}^{\prime}$ is shown for a typical choice of parameters: $\{\mathcal{L}, h, \Psi\}=\{200,0.5,0.99\}$.

The results shown in Table 7.3 highlight the importance of parameter optimization, and point out that by selecting the optimal parameters it is possible to improve the normalized MASE by a factor greater than two relative to arbitrary parameter selection. Optimization results are given for different fading channel models and for both a shadowed and an unshadowed scenario. Performance is very poor for the pessimistic assumption of Rayleigh fading, while it improves for a more realistic mixed fading, which implies that the source and destination are in the $\mathrm{LOS}$, while the other links are not. Shadowing is always detrimental, and even though it leads to a higher code rate, it requires a larger number of frequency channels. While the optimal modulation index and fractional in-band power vary with the density of interference per unit area and the distance of the transmitter from the receiver, similar to Table 7.2 they remain constant under different channel models and shadowing conditions. An increment in the density of interfering devices per unit area leads to an increment of 
the normalized MASE, as well as to a higher code rate, number of frequency channels, and fractional in-band power and a lower modulation index.

\subsection{Chapter Summary}

When used with coded CPFSK, the performance of FH ad hoc networks depends on the modulation index, the code rate, the number of frequency channels, and the fractional in-band power $\psi$. These parameters are often chosen arbitrarily.

This chapter describes a new optimization method for analyzing and optimizing $\mathrm{FH}$ ad hoc networks in the presence of Nakagami fading and shadowing with interfering devices drawn from an arbitrary point process which can cause both co-channel and adjacent channel interference. Under two different rate allocation policies, it has been shown here how to properly optimize the modulation format (including modulation index, number of frequency channels, and fractional-in-band power), and chose the appropriate code rate in order to maximize the throughput of the network. In particular, it has been shown that the MASE can be improved of a factor of 5-10 times when a common rate can be used, and of a factor of 2 times when rate adaptation is performed, compared to a scenario where a typical set of parameters is used under the same rate allocation policies.

In both cases, it has been highlighted the importance of adequately matching the bandwidth of the frequency channels to a certain percent power bandwidth of the modulation, which is typically and arbitrarily chosen to be 99-percent. In fact, it is possible to increase the bit rate while fixing the channel bandwidth, thereby decreasing the fractional in-band power and increasing the fraction of the signal power that splatters into adjacent channels. While the resulting increased $\mathrm{ACI}$ will negatively affect performance, the increased bit rate can be used to support a lower-rate error-correction code, thereby improving performance. 


\section{Chapter 8}

\section{Geographic Routing Protocols in Ad Hoc Networks}

In the past few years, there has been extensive research directed toward providing insights into the tradeoffs among the reliabilities, delays, and throughput of such networks with multi-hop routing (e.g., [128 134]). However, the mathematical models and necessary assumptions have not been adequate for obtaining reliable results. This chapter introduces a novel methodology 135 137 to evaluate the performance of multi-hop routing in ad hoc networks, in which many unrealistic assumptions and restrictions of existing analyses are discarded, such as: 1) absence of shadowing; 2) the neglect of thermal noise; 3) identical fading conditions for each link; 4) the independence of the success probabilities of path from the source to the destination even when paths share the same links; 5) the limiting of the number of end-to-end retransmissions rather than link retransmissions.

The methodology is characterized by a dual method, composed of a closed-form analysis, which follows from Chap. 2, and a lightweight simulation. While the methodology described here can be used to evaluate the performance for a very wide range of routing protocols, this chapter focuses on the performance of two representative geographic routing protocols 138. Furthermore, a comparison is made with the popular ad hoc on-demand distance-vector (AODV) routing protocol 139 to gain perspective about the advantages and disadvantages of geographic routing. The effects of various system parameters are illustrated for finite ad hoc networks with randomly placed devices. 


\subsection{Network Model}

Consider a DS-CDMA network of asynchronous quadriphase direct-sequence systems, composed of a source and a destination, which are indicated respectively by $X_{0}$ and $X_{M+1}$, and $M$ devices, which are potentially relays or sources of interference. Define $\mu_{i}$ to be the service probability that device $i$ can serve as a relay along a path from a source to a destination. With probability $p_{i}$, the $i^{\text {th }}$ device transmits in the same time interval as the desired signal. The $\left\{p_{i}\right\}$ can be used to model voice-activity factors, controlled silence, or failed link transmissions and the resulting retransmission attempts. When the $i^{\text {th }}$ device is in service as a potential relay, $p_{i}=0$ is set. Denote by $X_{o}$ a reference transmitter which which attempts to communicate with device $X_{j}$, and denote by $\mathcal{X}$ the set of devices, which uses the same radio resources than device $X_{o}$. By accounting for shadowing and a distance-dependent path loss model, the normalized received power is

$$
\Omega_{i, j}= \begin{cases}10^{\xi_{o, j} / 10} r_{o, j}^{-\alpha} & i=o \\ \frac{h P_{i}}{G P_{k}} 10^{\xi_{i, j} / 10} r_{i, j}^{-\alpha} & i \in \mathcal{X} \\ 0 & i \notin \mathcal{X}, i \neq o\end{cases}
$$

Under the assumption that the channel gains remain constant for the duration of a transmission, but vary from transmission to transmission, by substituting (8.1) in (2.9), it is possible to evaluate the conditional outage probability for a given transmission, when the topology of the networks is known as well as the activity of the other devices.

In this section, the devices are assumed to be distributed within a circular arena according with a uniform clustering model with exclusion zone $r_{e x_{m b}}$, while the source and destination devices are placed respectively at the center and at the distance $\left\|X_{M+1}-X_{0}\right\|$ from it. The distance-dependent-fading model given by 3.7 is adopted.

\subsection{Network Simulation}

The simulator is organized into four layers, each of which emulates a particular random feature of the network. The layers are implemented as nested for loops. The top layer handles the random network topology (i.e., the location of all devices in the network except for 
the source and destination, which are fixed); the second layer is concerned with classifying each device as either a potential source of interference or a potential relay; the third layer is concerned with determining which of the potential interferers actually transmits (assuming an Aloha protocol with random access probability $p_{i}$ is used), while the bottom layer determines which links are actually in an outage. The routing is also handled at the bottom layer.

During each iteration of the top layer, a network topology is generated by placing the locations of the devices according to the uniform clustering model. First the source and destination devices are placed in fixed positions, and then one by one, the location of each of the $M$ remaining $X_{i}$ is drawn according to a $\mathrm{UCP}$ within the network region. This loop is run $\Upsilon$ times, once per topology.

During each iteration of the second layer, which is run $K_{t 1}$ times, each device is independently marked as either being a potential relay (with probability $\mu_{i}$ ) or a potential source of interference. Those devices that are marked as potential relays cannot transmit interference, and are forced to have transmission probability $p_{i}=0$.

During each iteration of the third loop, which is run $K_{t 2}$ times, the set of transmitting interferers $\mathcal{X}$ is found for each time slot $t$, up to some maximum time. Each $\mathcal{X}$ is found by marking each potential interferer as transmitting by drawing a Bernoulli variable with probability $p_{i}$. Then, for each time slot $t$, a matrix is found containing the outage probabilities between all devices that may participate in the route (i.e., all devices except for the potential interferers).

During each iteration of the bottom layer, which is run $K_{t 3}$ times, each link is marked as either being in an outage or not for each time slot by drawing a Bernoulli variable with probability equal to the corresponding entry in the channel outage matrices. Once the links are marked as being in an outage or not, the set of links that may be used to support a route is identified, and the corresponding routing protocols (described in the next subsection) may be implemented. Counters are updated to keep track of the key network performance metrics. 


\subsection{Interference Correlation}

For a given network topology and service model, there is a common set of devices that may produce interference. These devices are in locations that are relatively fixed when compared to the timescales of communications. Because of the common randomness in the locations of potentially interfering devices, there is correlation in the interference. The correlation is both temporal, because subsequent interfering transmissions come from subsets of the same common set of devices, and spatial, due to the common locations of interfering transmissions within a given time slot. The spatio-temporal correlation exists even when the devices use Aloha as the MAC protocol, which produces transmissions that are locally uncorrelated. While interference correlation is usually neglected, it has become a subject of recent interest [16].

The network model and simulator presented here naturally accounts for the interference correlation. This is because during each iteration of the top layer of the simulation, the topology is fixed. Hence, the interfering devices selected at the third layer of the simulation is constrained to be drawn from this set. It follows that the calculation of outage probability properly accounts for interference correlation ${ }^{1}$.

\subsection{Routing Protocols}

The three routing protocols that are considered are reactive or on-demand protocols that only seek paths from the source to the destination when needed and do not require devices to store details about large portions of the network.

The AODV protocol uses an on-demand approach for finding a route during its pathdiscovery phase, which relies on flooding to seek the fewest-hops path, which is the path with the smallest number of links or hops. The flooding diffuses request packets simultaneously

\footnotetext{
${ }^{1}$ Note that here the simulator assumes a simple Aloha protocol which is used by each potential interferer. However, other devices in the network are likely to be participating in some other, unknown, route. The protocol may be the same or different than the protocol used by the reference route. While it is possible to extend the methology herein to simulate the separate routing action of neighboring nodes, allowing to capture the temporal correlation more accurately, this would require assumptions to be made about the behavior of the other nodes. In alternative, in order to capture the effect of temporal correlation, the value of the transmission probability can be found by opportunely fitting it with measured data.
} 
over multiple routes for the purpose of discovering a successful path to the destination despite link failures along some potential paths. When the first request packet reaches the destination, an acknowledgement is sent back to the source using the temporary fewest-hops path discovered and each subsequent reception of request packets is ignored. By using this route, the source sends subsequent message packets to the destination during a messagedelivery phase. This protocol has a very high overhead cost in establishing the fewest-hops path during the path-discovery phase, and furthermore the fewest-hops path must be used for message delivery before changes in the channel conditions cause an outage of one or more of its links.

Geographic protocols limit information-sharing costs by minimizing the reliance of devices on topology information $138,140,142$. Since geographic routing protocols make routing decisions on a hop-by-hop basis, they do not require a flooding process for path discovery. Two geographic routing protocols are examined: the greedy forwarding (GF) protocol and the maximum progress (MP) protocol. Both geographic routing protocols assume that each device knows its physical location and the direction towards the destination.

The GF protocol relies on beacons, which are periodic brief messages exchanged among devices that serve to identify neighboring active devices and their locations. Under the GF protocol, a source forwards a packet to a relay that is selected from a set of neighboring active devices that lie within a transmission range of radius $r_{\mathrm{t}}$. The next link in the path from source $X_{0}$ to destination $X_{M+1}$ is the link to the relay within the transmission range that shortens the remaining distance to $X_{M+1}$ the most. There is no path-discovery phase because the relays have the geographic information necessary to route the messages to the destination.

The principal problems with beacons are that their overhead cost is high, they generate additional interference that degrades data packets, and the location-information may become outdated. With beaconless routing, these problems are substantially reduced. Mobiles broadcast short request messages during a path-discovery phase only when they are ready to transmit data packets. The responses to the request messages reveal the identity and location of a neighboring active device that is suitable as the next relay during the message-delivery phase. 
The MP protocol is a contention-based beaconless protocol that comprises alternating path-discovery phases and message-delivery phases. During a path-discovery phase, a single link to a single relay is discovered. During the following message-delivery phase, a packet is sent to that relay, and then the alternating phases resume until the destination is reached. In a path-discovery phase, the next relay in a path to the destination is dynamically selected at each hop of each packet and depends on the local configuration of available relays. A source or relay broadcasts $R T S$ messages to neighboring active devices that potentially might serve as the next relay along the path to the destination. The RTS message includes the location of the transmitter. Upon receiving the $\mathrm{RTS}$, a neighboring device initiates a timer that has an expiration time proportional to the remaining distance to the destination. When the timer reaches its expiration time, the device sends a CTS message as an acknowledgement packet to the transmitter. The earliest arriving CTS message causes the source or previous relay to launch the message-delivery phase by sending message packets to the device that sent that CTS message, and all other candidate devices receiving that CTS message cease operation of their timers. The primary advantage of the RTS and CTS messages is that they can be used to establish guard zones $r_{\mathrm{g}}$ surrounding the transmitter and receiver [143]. Potentially interfering devices that receive one of these messages within a guard zone are silenced during the acknowledgement and message-delivery phases of the $\mathrm{MP}$ protocol. While it is possible to transmit the data directly, without first going through the RTS/CTS phase, the unsilenced interference is likely to cause an outage, therefore significantly degrading the throughput.

\subsection{Implementation of Path Selection}

Consider those protocols that have a path-discovery phase; i.e, AODV and MP routing. A candidate link is a link that does not experience an outage during the path-discovery phase. For AODV, the candidate paths from $X_{0}$ to $X_{M+1}$ are paths that can be formed by using candidate links. The candidate path with the fewest hops from $X_{0}$ to $X_{M+1}$ is selected as the fewest-hops path. This path is determined by using the Djikstra algorithm [144] with the unit cost of each candidate link. If two or more candidate paths have the fewest hops, the fewest-hops path is randomly selected from among them. If there is no set of candidate 
links that allow a path from $X_{0}$ to $X_{M+1}$, then a routing failure occurs and is recorded. If a fewest-hops path exists, then a Monte Carlo simulation is used to determine whether the acknowledgement packet traversing the path in the reverse direction is successful. If it is not or if the message delivery over the fewest-hops path fails, then a routing failure is recorded.

The geographic routing protocols have knowledge of the direction towards the destination, and a distance criterion is used to exclude a link from device $X_{i}$ to device $X_{j}$ as a link in one of the possible paths from $X_{0}$ to $X_{M+1}$ if $\left\|X_{j}-X_{M+1}\right\|>\left\|X_{i}-X_{M+1}\right\|$. In fact, these exclusions ensure that each possible path has links that always reduce the remaining distance to the destination. All links connected to devices that cannot serve as relays are excluded as links in possible paths from $X_{0}$ to $X_{M+1}$. Links that have not been excluded are called eligible links.

For the GF protocol there is no path-discovery phase, and the eligible links are used to determine the GF path from $X_{0}$ to $X_{M+1}$ during its message-delivery phase. If no path from $X_{0}$ to $X_{M+1}$ can be found or if the message delivery fails, a routing failure is recorded.

A two-way candidate link is an eligible link that does not experience an outage in either the forward or the reverse direction during the path-discovery phase. A Monte Carlo simulation is used to determine the two-way candidate links. For the maximum progress protocol, the two-way candidate link starting with source $X_{0}$ with a terminating relay that minimizes the remaining distance to destination $X_{M+1}$ is selected as the first link in the MP path. The link among the two-way candidate links that minimizes the remaining distance and is connected to the relay at the end of the previously selected link is added successively until the destination $X_{M+1}$ is reached and hence the MP path has been determined. After each relay is selected, a message packet is sent in the forward direction to the selected relay. If no MP path from $X_{0}$ to $X_{M+1}$ can be found or if a message delivery fails, a routing failure is recorded.

Each RTS or CTS message transmitted by the MP protocol during its path-discovery phase establishes a guard zone. If a candidate link exists from the source of an RTS or CTS message to a potentially interfering device within the source's guard zone, then that device is silenced during the acknowledgement and message-delivery phases. Silencing of a device is modeled by removing it from the set $\mathcal{X}$. Since the $\mathrm{MP}$ protocol is a geographic protocol, 
potentially interfering devices know how far they are from the source of an RTS or CTS message. If they are beyond the guard zone, then they can ignore the message and continue their own transmissions.

In device ad hoc networks, the fading processes affecting different links of the same path are not significantly correlated for two reasons. The exclusion zones surrounding the two receivers ensure a significant physical separation. As a result, the two receivers have much different multipath environments and hence experience largely uncorrelated fading. The second reason is that the time between transmissions over successive links in a path usually exceeds the channel coherence time. These two factors decorrelate the fading over different links of the same path.

\subsection{Performance Metrics}

Let $N_{\text {tr }}$ denote the maximum number of transmission attempts over a link of the path. During the path-discovery phases, $N_{\mathrm{tr}}=1$. During the message-delivery phases, $N_{\mathrm{tr}} \geq 1$ because message retransmissions over an established link are feasible. For each eligible or candidate link $l=(i, j)$, a Bernoulli random variable with failure probability $\epsilon_{l}$ is repeatedly drawn until there are either $N_{\text {tr }}$ failures or success after $N_{l}$ transmission attempts, where $N_{l} \leq N_{\text {tr. }}$. The delay of link $l$ of the selected path is $N_{l} \mathcal{D}_{\text {tol }}+\left(N_{l}-1\right) \mathcal{D}_{e}$, where $\mathcal{D}_{\text {tol }}$ is the delay of a transmission over a link, and $\mathcal{D}_{e}$ is the excess delay caused by a retransmission.

Each network topology $t$ is used in $K_{t}$ simulation trials. The path delay $\mathcal{D}_{s, t}$ of a path from $X_{0}$ to $X_{M+1}$ for network topology $t$ and simulation trial $s$ is the sum of the link delays in the path during the message-delivery phase:

$$
\mathcal{D}_{s, t}=\sum_{l \in \mathcal{L}_{s, t}}\left[N_{l} \mathcal{D}_{t o l}+\left(N_{l}-1\right) \mathcal{D}_{e}\right]
$$

where $\mathcal{L}_{s, t}$ is the set of links constituting the path. If there are $N_{\mathrm{tr}}$ transmission failures for any link of the selected path, then a routing failure occurs.

If there are $F_{t}$ routing failures for topology $t$ and $K_{t}$ simulation trials, then the probability of end-to-end success or path reliability within topology $t$ is

$$
\mathcal{R}^{(t)}=1-\frac{F_{t}}{K_{t}} .
$$


Let $\mathcal{T}_{t}$ denote the set of $K_{t}-F_{t}$ trials with no routing failures. If the selected path for trial $s$ has $h_{s, t}$ links or hops, then among the set $\mathcal{T}_{t}$, the average conditional number of hops from $X_{0}$ to $X_{M+1}$ is

$$
\mathcal{H}^{(t)}=\frac{1}{K_{t}-F_{t}} \sum_{s \in \mathcal{T}_{t}} h_{s, t} .
$$

Let $\mathcal{D}_{\text {pdp }}$ denote the link delay of packets during the path-discovery phase. The average conditional delay from $X_{0}$ to $X_{M+1}$ during the combined path-discovery and message-delivery phases is

$$
\mathcal{D}^{(t)}=\frac{1}{K_{t}-F_{t}} \sum_{s \in \mathcal{T}_{t}}\left(\mathcal{D}_{s, t}+2 d h_{s, t} \mathcal{D}_{\mathrm{pdp}}\right)
$$

where $d=0$ for the GF protocol, and $d=1$ for the MP and AODV protocols.

Given the density of the possible transmitters in the network $\lambda=(M+1) /|\mathcal{A}|$, define the normalized $A S E$ for the $K_{t}$ trials of topology $t$ as

$$
\mathcal{S}_{\mathrm{ase}}^{(t)}=\frac{\lambda}{K_{t}} \sum_{s \in \mathcal{T}_{t}} \frac{1}{\mathcal{D}_{s, t}+2 c h_{s, t} \mathcal{D}_{\mathrm{pdp}}}
$$

where the normalization is with respect to the bit rate or bits per channel use. The normalized ASE is a measure of the maximum end-to-end throughput in the network. After computing $\mathcal{R}^{(t)}, \mathcal{H}^{(t)}, \mathcal{D}^{(t)}$, and $\mathcal{S}_{\text {ase }}^{(t)}$ for $\Upsilon$ network topologies, the topological averages: $\overline{\mathcal{R}}$, $\overline{\mathcal{H}}, \overline{\mathcal{D}}$, and $\overline{\mathcal{S}}_{\text {ase }}$ can be evaulated averaging over a large set of topologies.

The average values of the interference probability and the service probability are defined as

$$
\bar{p}=\frac{1}{M} \sum_{i=1}^{M} p_{i}, \quad \bar{\mu}=\frac{1}{M} \sum_{i=1}^{M} \mu_{i}
$$

respectively. The contention density $\lambda \bar{p}$ is a measure of the average number of interfering devices and the average number of simultaneous transmitted packets. The relay density $\lambda \bar{\mu}$ is a measure of the average number of available relays.

\subsection{Performance Analysis}

Consider a network with $M=200$ devices distributed within a circular arena with normalized radius $r_{\text {out }}=1$. Delays are normalized by setting $\mathcal{D}_{\text {tol }}=1, \mathcal{D}_{e}=1.2$, and $\mathcal{D}_{\text {pdp }}=0.1$. Assume the transmitted power $P_{i}$ is equal for all the devices. The exclusion zone 


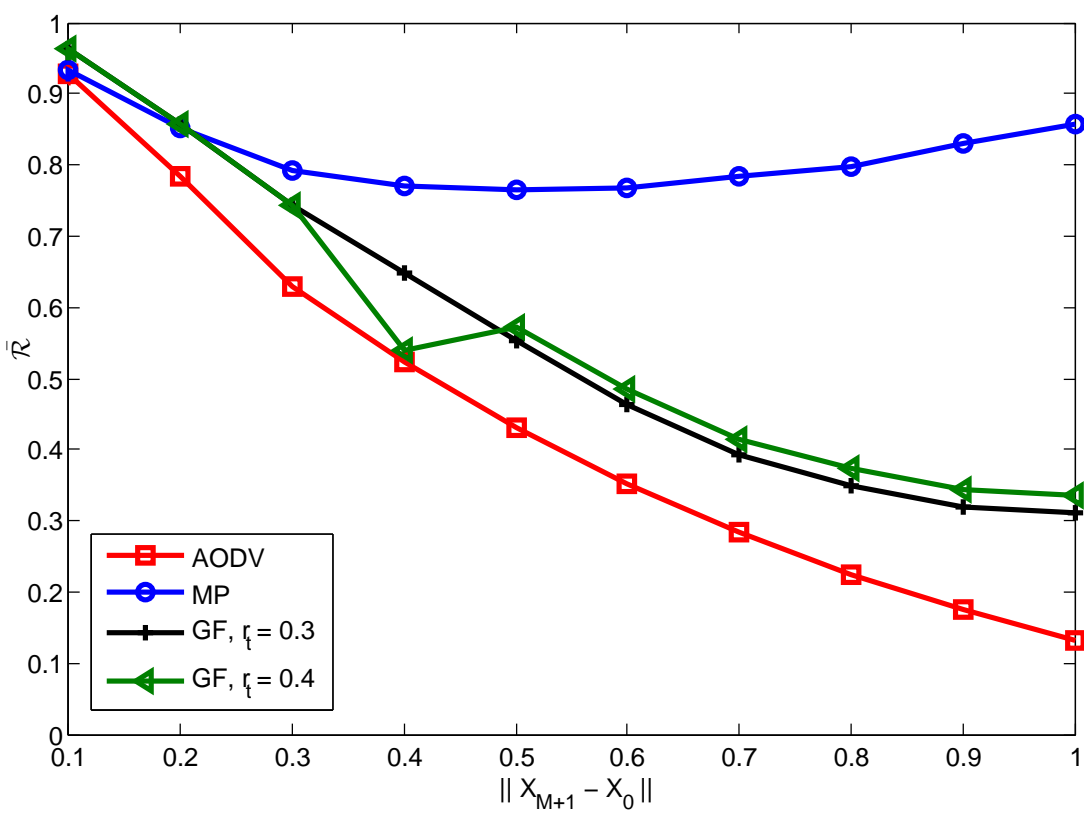

Figure 8.1: Average path reliability for both phases of each routing protocol as a function of the distance between source and destination.

for the devices is set to $r_{e x_{m b}}=0.05$ and the circular guard zones have radius $r_{\mathrm{g}}=0.15$. The SINR threshold is fixed to $\beta=0 \mathrm{~dB}, \mathrm{SNR}=0 \mathrm{~dB}$, and $\alpha=3.5$. The Monte Carlo simulations are run over $\Upsilon=2,000$ topologies, where $K_{t}=K_{t 1} K_{t 2} K_{t 3}=10^{6}\left(K_{t 1}=K_{t 2}=K_{t 3}=100\right)$. The service and interference probabilities are assumed to have the same values for all devices so that $\mu_{i}=\mu$ and $p_{i}=p$. Therefore, $\lambda \mu$ is the relay density, and $\lambda \bar{p}=\lambda p(1-\mu)$ is the contention density. Unless otherwise stated, $G / h=96, N_{\mathrm{tr}}=4, r_{\text {los }}=0.2, \mu=0.4$, and $p=0.3$. When shadowing is present, it has a lognormal distribution with $\sigma_{s}=8 \mathrm{~dB}$. However, the transmitted packets encounter the same shadowing in both directions over the same link during both routing phases.

Fig. 8.1 shows the overall average path reliabilities for the combined path-discovery and message-delivery phases of all three routing protocols. The AODV protocol is the least reliable. The $\mathrm{MP}$ protocol is much more reliable than the $\mathrm{GF}$ protocol if $\| X_{M+1}-$ $X_{0} \|$ is large, but it is not as reliable if $\left\|X_{M+1}-X_{0}\right\| \leq 0.2$ because of the relatively low reliability of its request packets. The not monotonic behavior of the path reliability for the MP protocol occurs because at short distances, there are very few relays that provide forward progress, and often the only candidate or two-way candidate link is the direct link 


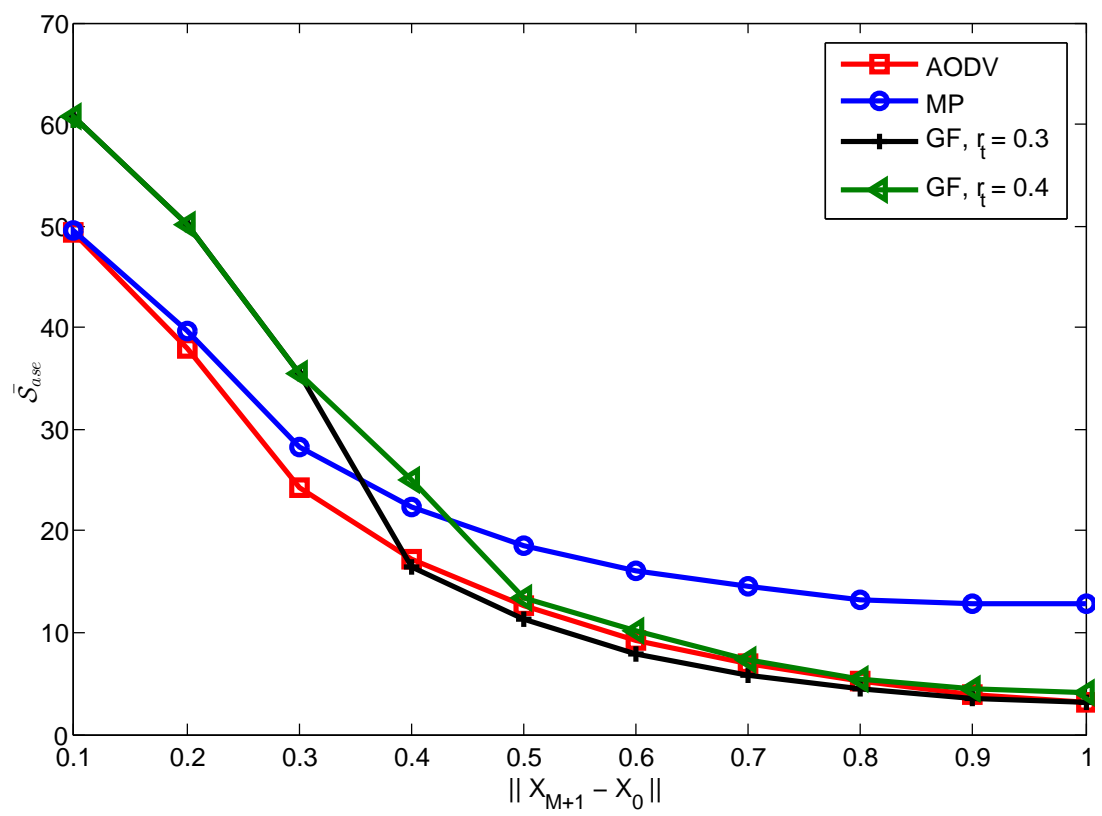

Figure 8.2: Normalized ASE of each routing protocol as a function of the distance between source and destination.

from source to destination. As the distance increases, there are more candidate and two-way candidate links, and hence the network benefits from the diversity. Furthermore, as the destination approaches the edge of the network, the path discovery benefits from a decrease in interference at the relays that are close to the destination.

The normalized ASE $\overline{\mathcal{S}}_{\text {ase }}$ for each routing protocol as a function of the distance between the source and the destination is displayed in Fig. 8.2. The GF protocol has the highest normalized ASE if $\left\|X_{M+1}-X_{0}\right\|$ is small, whereas the MP protocol has the highest if $\left\|X_{M+1}-X_{0}\right\|$ is large. The reason is that the GF protocol needs only one hop for a message to reach the destination when $\left\|X_{M+1}-X_{0}\right\|$ is small, whereas this protocol has reduced reliability and significantly increased average conditional delay, as the distance between the source and the destination is large.

The average conditional delay $\overline{\mathcal{D}}$ and the normalized ASE $\overline{\mathcal{S}}_{\text {ase }}$ for each routing protocol as a function of the maximum number of retransmissions during the message-delivery phase when $\left\|X_{M+1}-X_{0}\right\|=0.5$ are displayed in Fig. 8.3. The GF protocol has a monotonically increasing average conditional delay as the maximum number of retransmissions allowed increases because new paths from the source to the destination with longer delays become 


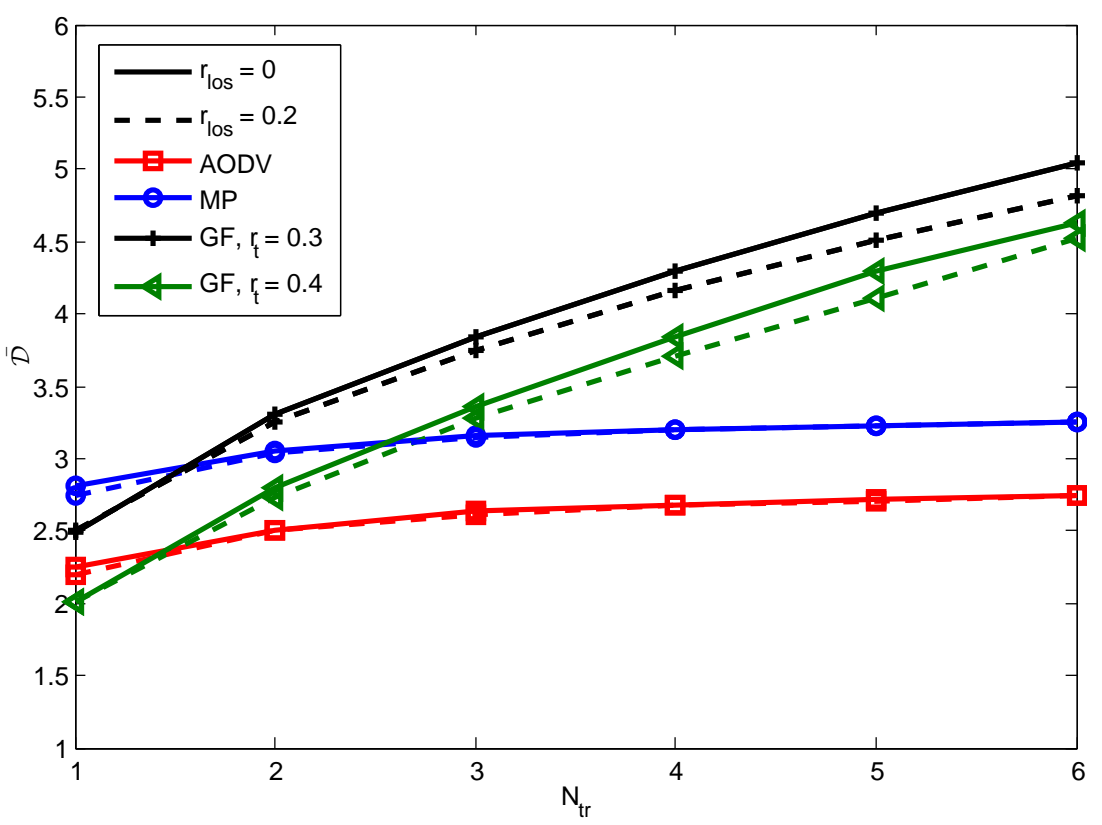

(a) Average conditional delay

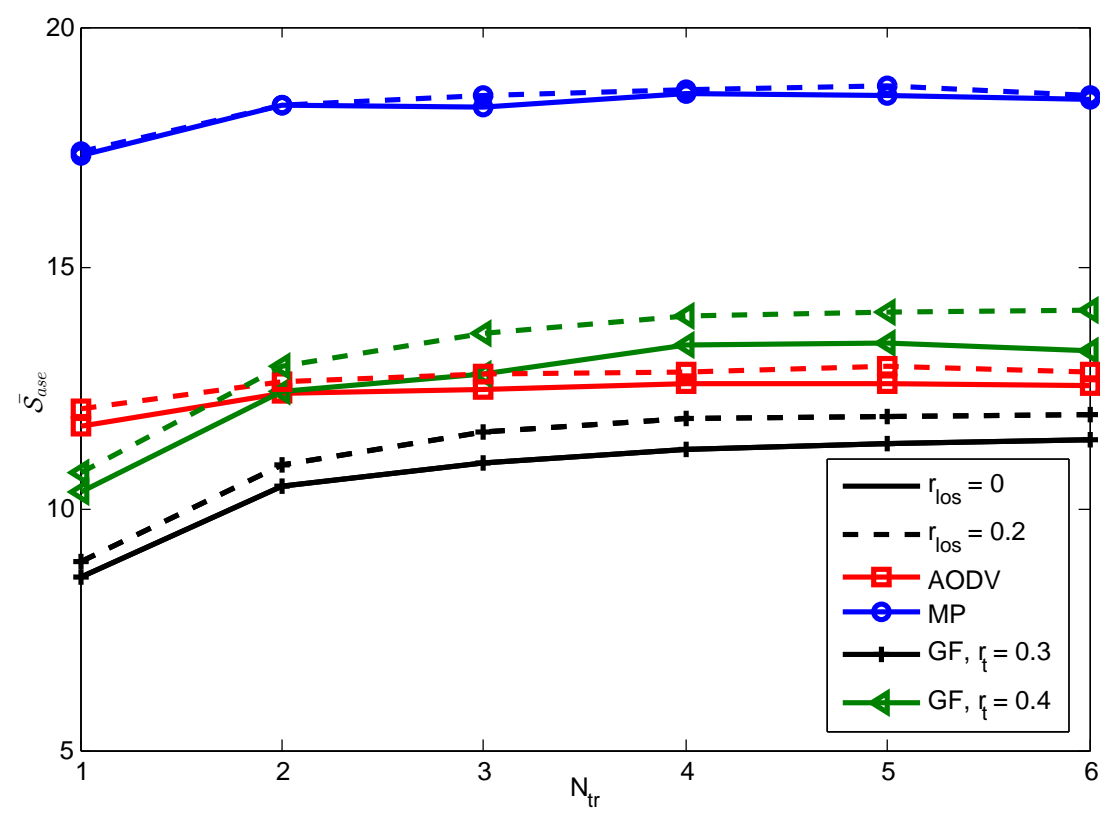

(b) Normalized area spectral efficiency

Figure 8.3: Average conditional delay and normalized ASE of each routing protocol as a function of the maximum number of retransmissions during the message-delivery phase

viable. However, an increase in the maximum number of retransmissions allowed has little effect on the average conditional delay for the AODV and MP protocols. An increase from $N_{\mathrm{tr}}=1$ to $N_{\mathrm{tr}}=2$ produces an increase in the normalized ASE for all three protocols, 


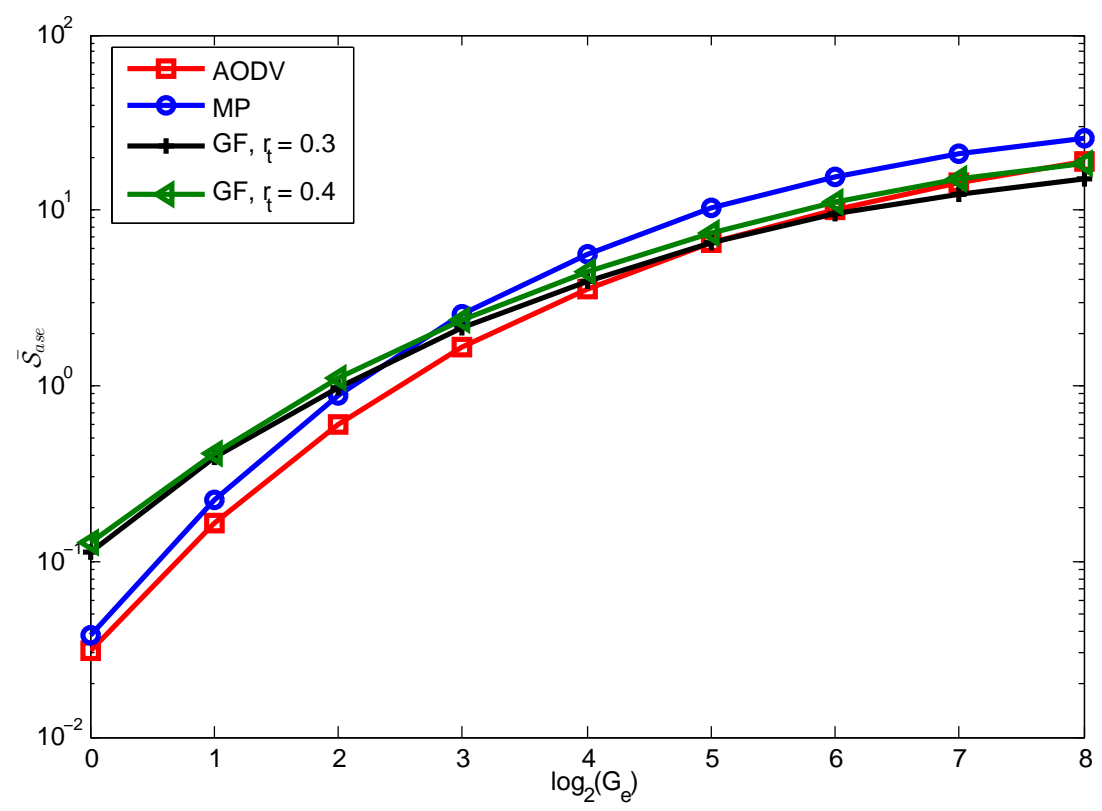

Figure 8.4: Normalized ASE of each routing protocol as a function of the effective spreading factor.

but further increases produce only a minor improvement. The reason is that the higher path reliabilities are offset by the increased numbers of successful paths with longer delays. Fig. 8.3 illustrates the losses incurred by the protocols when the fading is the more severe Rayleigh fading $\left(r_{\text {los }}=0\right)$ instead of mixed fading.

The critical role of the spreading factor in suppressing interference is illustrated in Fig. 8.4 for $\left\|X_{M+1}-X_{0}\right\|=0.5$. The normalized ASE of each protocol increases monotonically with the effective spreading gain $G_{e}$ but the rate of increase is greatest for the MP protocol. In the absence of spreading, the GF protocol provides the largest normalized ASE.

Fig. 8.5 shows the normalized ASE for each routing protocol when $\left\|X_{M+1}-X_{0}\right\|=0.5$ as a function of the contention density $\lambda \bar{p}$ with the relay density $\lambda \mu$ as a parameter. This figure indicates the degree to which an increase in the contention density is mitigated by an increase in the relay density. Fig. 8.5 was generated by varying $p$ and $\mu$ while maintaining $M=200$. However, nearly the same plots are obtained by varying $M$ or $\lambda$ while maintaining $p=0.3$ and $\mu=0.4$. Thus, the contention density and relay density are of primary importance, not the individual factors $p, \mu$, and $\lambda$.normalized ASE than the MP protocol. The GF protocol with $r_{t}=0.3$ has a poor normalized ASE when the relay density is low because of the paucity 


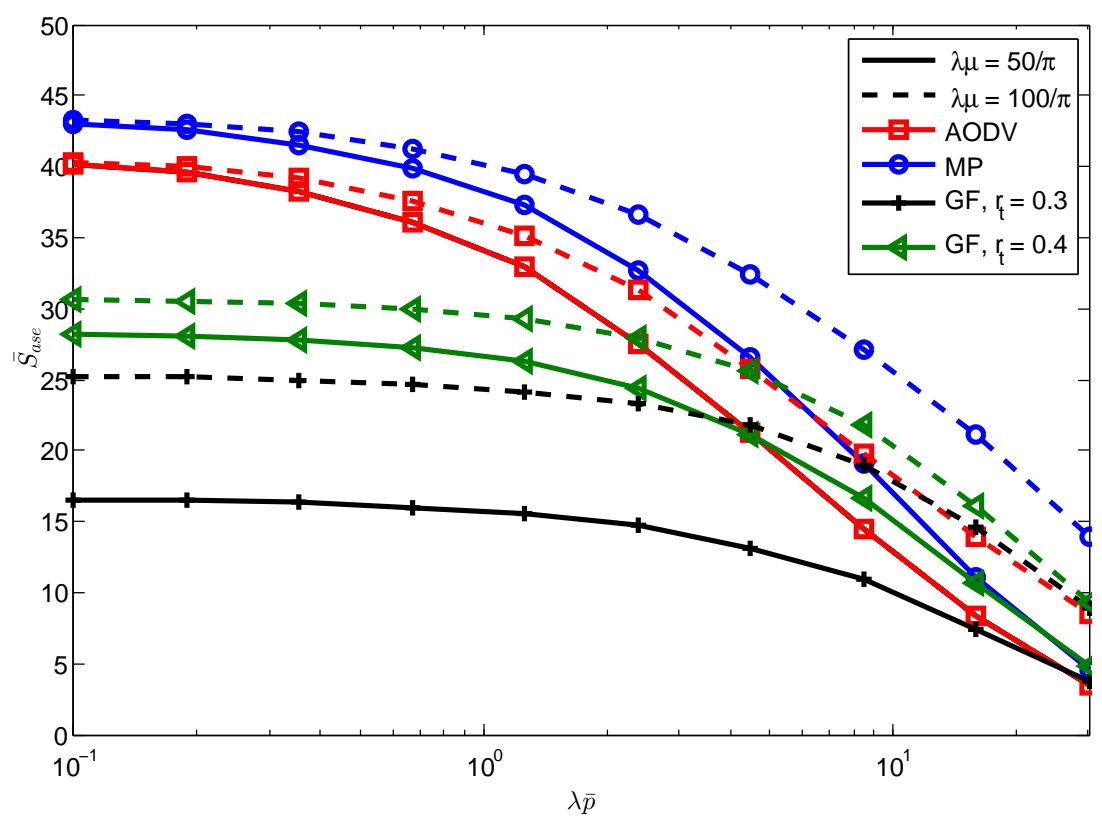

Figure 8.5: Normalized ASE for each routing protocol as a function of the contention density with the relay density as a parameter.

of potential relays within its transmission range. When $r_{t}=0.4$ and both the relay density and contention density are large, the GF protocol provides a normalized ASE superior to that of the AODV protocol and approaching that of the MP protocol.

\subsection{Chapter Summary}

This chapter has presented a new approach for modeling and characterizing the statistical performance of multi-hop routing ad hoc networks. This methodology can be used to provide a significantly improved analysis of multi-hop routing protocols in ad hoc networks. Many unrealistic and improbable assumptions and restrictions of existing analyses can in fact be discarded. In this chapter, the performance of two representative geographic routing protocols are evaluated for large ad hoc networks with randomly placed devices. Furthermore, a comparison is made using as a benchmark the popular AODV routing protocol to gain perspective about the advantages and disadvantages of geographic routing. However, the methodology described here is general enough that can be used to evaluate the performance for a very wide range of routing protocols. 


\section{Chapter 9}

\section{Stochastic Modeling of Cooperative Multi-hop Wireless Networks}

The use of Markov chains and their properties have been extensively used in the last decade to characterize the performance of a multi-hop cooperative ad hoc networks. However, in most related work the effect of the CCT is neglected [145, 146] or, simplified assumptions [147, which are accurate only for high density networks, are made in order to facilitate the analysis. Furthermore, the approach often used in literature to model and analyze a cooperative multi-hop network is very computational intense. The outage probability of each link is usually computed conditioning over a set of channel realizations, and then the average performance of the network is evaluated by taking the average over the fading, once the end-to-end outage probability is obtained.

After a brief overview on Markov process and its properties, this chapter proposes a novel framework [148] to stochastically model and analyze the performance of multi-hop cooperative networks. This framework is based on the analysis developed in Chap. 2 and 4. As will be more evident along the chapter, this framework reduces considerably the computational complexity of the problem, and also allows to account more accurately for the effect of the CCI, from other active nodes in the network. The chapter follows by applying the aforementioned framework to study the performance of unicast BRNs, and to optimize their performance in terms of ASE, when both the intra-controlled barrage region ( $\overline{\mathrm{CBR}}$ ) and inter-CBR interference are taken into account. For each source of interference, an iterative 
method is proposed in order to capture the temporal correlation and the interdependence between packets that propagate within the network.

\subsection{Markov Process and Multi-hop Wireless Networks}

Denote by $X(t)$ a random process. $X(t)$ is a Markov process if the future of the process given the present is independent of the past, and for instance,

$$
P\left[X(t+1)=x_{t+1} \mid X(t)=x_{t}, \cdots, X(0)=x_{0}\right]=P\left[X(t+1)=x_{t+1} \mid X(t)=x_{t}\right]
$$

where $x_{t}$ is the value assumed by the Markov process at time $t$.

If $X(t)$ is an integer-valued Markov process, it is usually also called Markov chain ${ }^{1}$. A Markov chain can be described as follows. Let $S=\left\{s_{1} ; s_{2} ; \ldots ; s_{r}\right\}$ be the set of states of the Markov chain. The process starts in one of these states and moves successively from one state to another. Each move is called a step. If the chain is currently in state $s_{i}$, then it moves to state $s_{j}$ at the next step with a probability denoted by $p_{i, j}$, and this probability does not depend upon which states the chain was in before the current state. The probabilities $p_{i, j}$ are called transition probabilities. The process can remain in the state it is in, and this occurs with probability $p_{i, i}$. An initial probability distribution, defined on $S$, specifies the starting state. Usually this is done by specifying a particular state as the starting state.

A state $s_{i}$ of a Markov chain is called absorbing if it is impossible to leave it (i.e., $p_{i, i}=1$ ). A Markov chain is absorbing if it has at least one absorbing state, and if from every state it is possible to go to an absorbing state (not necessarily in one step).

The Markov transition probabilities can then be placed into a state transition matrix $\boldsymbol{P}$, whose $(i, j)^{t h}$ entry is $\left\{p_{i, j}\right\}$. The Markov states are ordered so that the $\tau$ transient states and indexed before the $a$ absorbing states. With this ordering, the state transition matrix assumes the following canonical form 149

$$
P=\left[\begin{array}{ll}
Q & R \\
0 & I
\end{array}\right]
$$

\footnotetext{
${ }^{1}$ Note that in this chapter, since it is considered an integer-valued Markov process, the terms process and chain are used interchangeably.
} 
where $\boldsymbol{Q}$ is the $\tau \times \tau$ transient matrix, $\boldsymbol{R}$ is the $\tau \times a$ absorbing matrix and $\boldsymbol{I}$ is an $a \times a$ identity matrix.

Let $b_{i, j}$ be the probability that the process will be absorbed in the absorbing state $s_{j}$ if it starts in the transient state $s_{i}$. The absorbing probability $b_{i, j}$ is the $(i, j)^{t h}$ entry of the matrix $\boldsymbol{B}$, which can be computed by (see, for instance [149])

$$
B=N R
$$

where $\boldsymbol{N}=(\boldsymbol{I}-\boldsymbol{Q})^{-1}$ is the fundamental matrix.

In the context of a stochastic analysis of the performance of a cooperative multi-hop adhoc networks, a Markov chain can be used to describe their complete dynamics in time. In particular, once a finite set $S$ of states is defined, which include all the possible conditions of the cooperative network under examination, the transition probabilities for all these states is evaluated using the knowledge of the outage probability for each of the links that might compose the network. In order to reduce the computational complexity of the problem, the outage probability is evaluated using a similar approach proposed at the beginning of this dissertation, where the outage probability is computed directly by averaging over the fading, but conditioning over a specific topology of the network, by accounting also for the CCI. Specifically, if multiple source of transmissions are diversity combined at the receiver, the framework developed in Chap. 4 can be used, and in particular the outage probability can be evaluated by (4.9). Once the transition probabilities are computed and entered in the transition matrix as in $(9.2)$, the end-to-end outage probability of the network can be evaluated using $(9.3)$. If the absorbing states are indexed so that the first absorbing state corresponds to a network outage, while the second corresponds to a network success, and by assuming the network always starts in state $s_{1}, b_{1,1}$ corresponds to the end-to-end outage probability of the network, averaged over fading.

\subsection{Application: Barrage Relay Networks}

In this section, the previously described framework is applied as an example to model a BRN, After a brief overview on BRNs and $\overline{\mathrm{CBR}}$, this section follows by describing 
how to stochastically characterize the effect of both the inter-CBR as well as the intraCBR interference through an iterative method, which uses the Markov representation of the network. In both cases, the network is optimized for several scenarios based on a set of parameters, which includes the number of relays, the code rate at which a node transmits and the duration of a radio frame.

\subsubsection{Barrage Relay Network}

A $B R N$ is a simplified abstraction of a tactical mobile ad hoc network (MANET) architecture that is currently being used in the field [150,151]. BRN employ time division multiple access (TDMA $)$ and cooperative communications. All nodes utilize a common frame format that requires coarse slot-level synchronization. This can be accomplished via a distributed network timing protocol when a satellite reference is unavailable. BRNs use autonomous cooperative communications rather than distributed beamforming or space-time coding to (i) minimize the communications overhead required for cooperation and (ii) enable multiple transmitters to participate in cooperative links to multiple destinations without knowing which other nodes are transmitting. As detailed in [152], each node applies an independent, random phase-dithering pattern to each relayed message. This induces a time-varying fading characteristic at each receiver. By changing the phase dither within a packet, destructive interference across an entire packet can be avoided. When modern codes with long block lengths are used, the phase-dithering technique effectively translates spatial diversity from multiple transmitters into time diversity that can be captured via maximal-likelihood sequence estimation (MLSE) equalization and iterative detection.

The BRN flooding primitive works as follows. During the first slot of a frame, the source broadcasts its message. During the second slot, any node that received and successfully decoded the initial transmission will rebroadcast it. If more than one node concurrently transmits the message, then the superposition of their signals is received. The diverse signal components are effectively maximal-ratio combined at each receiver. During each subsequent slot, all messages that were successfully decoded during the previous slot are rebroadcasted. The process repeats until either the destination is reached, no receiver successfully decodes 


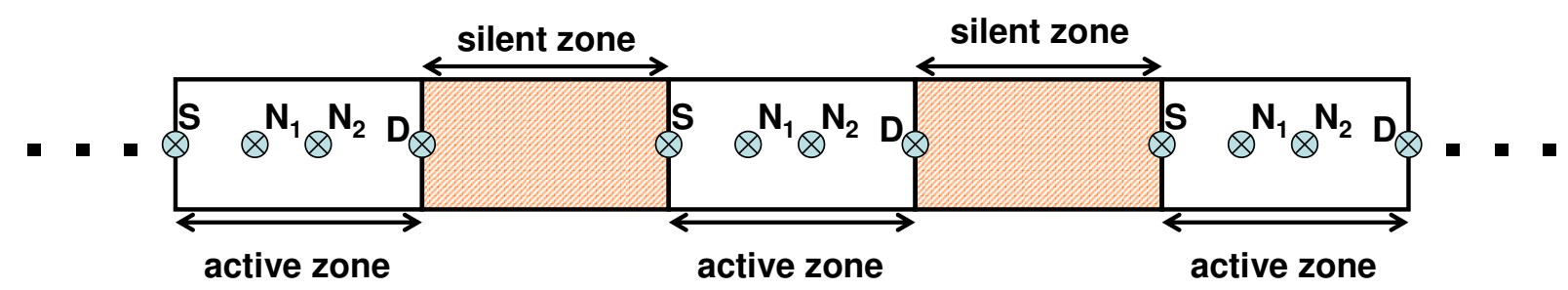

Figure 9.1: Example of Barrage relay networs (BRNs), composed of multiple controlled barrage regions (CBRs). Each CBR is composed a four-node network.

a transmission, or a maximum number of transmissions is reached. Packets thus propagate outward from the source via a decode-and-forward approach. To prevent relay transmissions from propagating back towards the source, each node relays a given packet only once.

\subsubsection{Controlled Barrage Regions}

In a traditional network, a link is defined by a transmit/receive radio pair that share a suitably reliable point-to-point communications channel. A unicast route is simply a series of these links connecting a source-destination node pair. In a BRN, however, links are cooperative and comprise multiple transmitters and receivers. A cooperative path is made up of series of cooperative links between the source and destination nodes. ACBR is simply the union of one or more such cooperative paths within some subregion or zone of the overall network. CBR $;$ afford a mechanism for unicast transport that is more robust than both traditional unicast routing and non-cooperative multipath variants [153.

CBRs can be established by specifying a set of buffer nodes around a set of cooperating interior nodes. Each buffer node acts as the source for transmissions into a $\overline{C B R}$ and the destination for transmissions emanating from within the CBR. External packets may enter the $\mathrm{CBR}$ only through a buffer node, and packets internal to a $\mathrm{CBR}$ may only propagate to the rest of the network through a buffer node. In this way, multiple unicast transmissions may be established in different portions of the network. This is referred to as spatial reuse. The buffer and interior nodes corresponding to a given source-destination pair can be specified via the broadcast of RTS / CTS packets [153].

An example of linear $\overline{B R N}$ is shown in Fig. 9.1. Each $\overline{C B R}$ in the example is made up of 
four nodes. The two interior nodes of each CBR, $R_{1}$ and $R_{2}$, act as relays, while the other two nodes are buffers, acting as the source $S$ and destination $D$ of the transmissions within the CBR, respectively. In the case of a linear BRN, each CBR is defined to be a segment of length $d$, here assumed to be the same for all CBR.

Let $N$ be the number of interior nodes in a CBR. Let $F$ be the number of slots of which a radio frame is composed. Given a packet that is injected within a CBR, since each node transmits each packet at most once and since each transmission requires one slot, it requires at most $N+1$ time slots to be received by the buffer node on the other side of the CBR in which the transmission has initially taken place. If $F<N+1$, the initial packet transmitted might require more than a radio frame to reach the destination. In this case, since a new packet may be broadcasted by the source of a CBR during only the first time slot of a radio frame, a intra-CBR collision takes place due to the fact that two or more packets might simultaneously propagate within the same CBR.

In ideal channel conditions, it is possible that the destination receives the initial source transmission in the first slot, though more generally, additional transmissions from the interior nodes may be required for successful reception. The buffer node acting as the source for one $\mathrm{CBR}$ also acts as the destination for the previous CBR, Because the nodes operate in a half-duplex mode, it is not possible for the buffer to simultaneously transmit into one CBR while receiving from another CBR, Thus, it is advisable to only allow one of the two CBR serviced by a given buffer node to be active; i.e., either the CBR that the buffer node is transmitting into is active or the $\overline{C B R}$ that the buffer node is receiving from is active, but not both. More generally, this suggests that CBR san be divided into two types during a particular radio frame: 1) active zones and 2) silent zones. During a given radio frame, only the nodes in active zones may transmit. As illustrated in Fig. 9.1, zones will typically alternate between active zones and silent zones, though it is feasible to have even further spatial separation between active zones. A key advantage to alternating between active and silent zones is that it provides spatial separation between adjacent active CBRs, which produce inter-CBR interference to each other. 


\subsubsection{Network Model}

Consider a $\mathrm{BRN}$ composed of $K \overline{\mathrm{CBR}}$. The $\mathrm{BRN}$ is a set of $M$ mobile radios or nodes $\mathcal{X}=\left\{X_{1}, \ldots, X_{M}\right\}$. As in the previous chapters, the variable $X_{i}$ represents both the $i^{\text {th }}$ node and its location. During the $t^{t h}$ time slot of a frame, $t \in\{1, \ldots, F\}$, node $X_{i}$ transmits with probability $p_{i}^{(t)}$. Any node in a silent zone will be characterized by $p_{i}^{(t)}=0$ for all slots of the frame. Since the source of an active CBR may only transmit in the first slot of the frame, it is characterized by $p_{i}^{(1)}=1$ and $p_{i}^{(t)}=0$ for $t \in\{2, \ldots, F\}$.

Let $\mathcal{X}_{j}^{(t)} \subset \mathcal{X}$ be the set of cooperating or barraging nodes that transmit identical packets to $X_{j}$ during the $t^{t h}$ time slot and $\mathcal{G}_{j}^{(t)}$ the set of the indexes of the nodes in $\mathcal{X}_{j}^{(t)}$. Let $\mathcal{N}_{j}=\left|\mathcal{X}_{j}\right|$ denote the number of cooperating nodes.

Nodes within the same CBR can transmit if a packet that was injected during a previous radio frame is still propagating, and in this case it causes intra-CBR interference. Nodes in other active $\overline{\mathrm{CBR}} 5$ are also potential sources of interference and cause inter-CBR interference. Each node that acts as an interferer will generate CCI with probability $p_{i}^{(t)}$. The value of $p_{i}^{(t)}$ for each of these nodes will depend on the dynamics of the protocol, as will be described shortly.

Under a distance-dependent path loss model, fading and in absence of shadowing, the outage probability conditioned over the network topology $\epsilon_{j}(\Omega)$ is given by 4.9 when the $\mathcal{N}_{j}$ cooperative transmissions are diversity combined at the receiver. Furthermore, in this case $\mathbb{P}\left[I_{i}=1\right]=p_{i}^{(t)}$ and the normalized received power at the $j^{\text {th }}$ node, when the $i^{\text {th }}$ node transmits, is

$$
\Omega_{i, j}= \begin{cases}r_{i, j}^{-\alpha} & i \in \mathcal{G}_{j} \\ \frac{P_{i, j}}{P_{0}} r_{i, j}^{-\alpha} & i \notin \mathcal{G}_{j}\end{cases}
$$

\subsubsection{Controlled Barrage Region as a Markov Process}

Consider a single packet being transmitted through a single CBR composed of a source (denoted $S$ ), a destination $(D)$ and $N$ relays $\left(\left\{N_{1}, \ldots, N_{N}\right\}\right)$. At the boundary between any two time slots, each node can be in one of the three following states, which is referred to as the node state: 


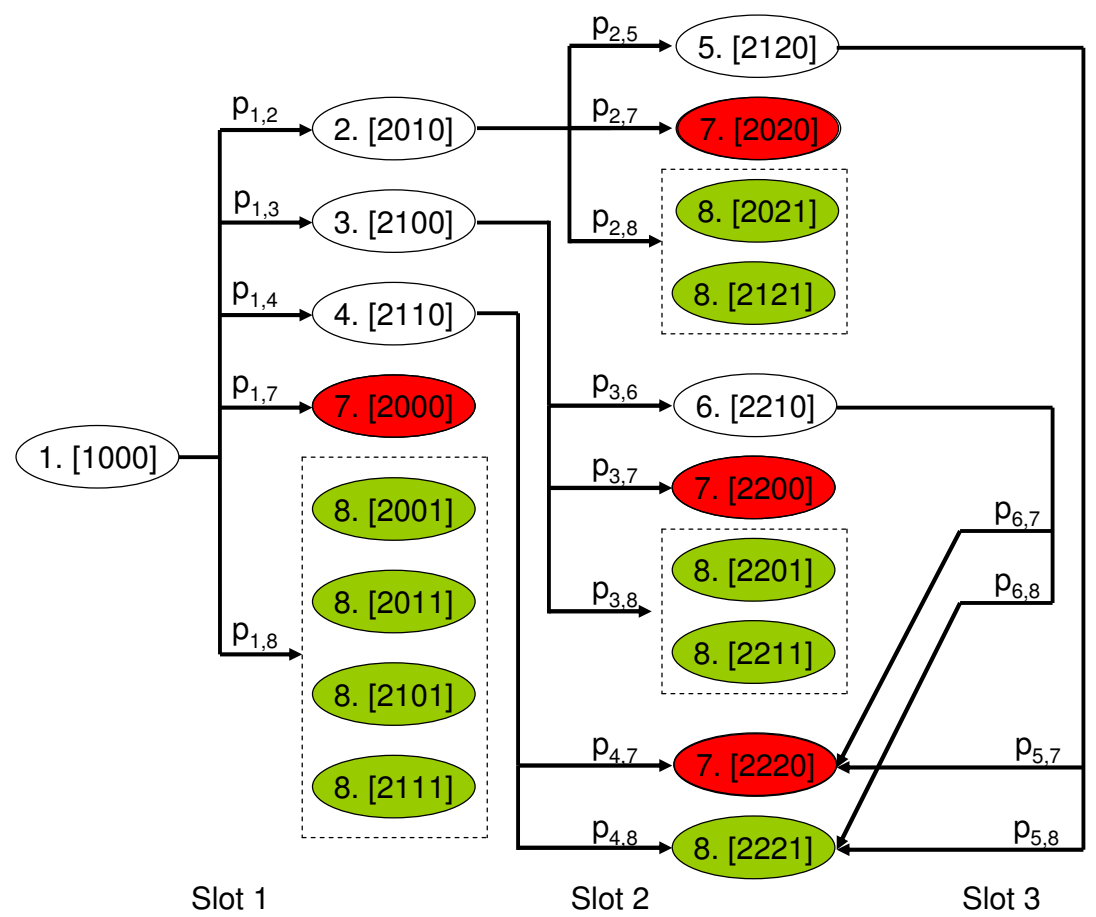

Figure 9.2: Markov chain for a CBR composed of four nodes $(N=2)$ and for the duration of three time slots. Transient states are in white, while the CBR success absorbing state is in green and the CBR outage absorbing state is in red. Each of the two absorbing states is the union of several CBR states.

- Node state 0: The node has not yet successfully decoded the packet.

- Node state 1: It has just decoded the packet received during the previous slot, and it will transmit on next slot.

- Node state 2: It has decoded the packet in an earlier slot and it will no longer transmit or receive that packet.

The state of the CBR is the concatenation of the states of the individual nodes within the CBR. Hereafter, it is referred to as the $C B R$ state. The CBR state can be compactly represented by a vector of the form $\left[S, N_{1}, \ldots, N_{N}, D\right]$ containing the states of the source, relays, and destination.

As previously mentioned, the behavior of the CBR can be described as an absorbing Markov process, which describes the dynamics of how the CBR states evolve over time ${ }^{2}$.

\footnotetext{
${ }^{2}$ Note that it is often possible to group several CBR states into a single Markov state. For instance, all of the CBR states are grouped corresponding to successful decoding by the destination into a single absorbing Markov state.
} 
Fig. 9.2 shows the Markov chain for a CBR with $N=2$ relays (a four-node network) for $F=N+1$ time slots, which is the maximum number of slots that might be used to successfully reach the destination. The CBR states are shown as a 4-element vector, along with the transition probabilities. The message is successfully delivered whenever the destination receives the message, which is indicated by a 1 in the last position of the CBRstate vector. Such states are marked in green, and hereafter this condition is referred as a CBR success. The transmission fails whenever all entries in the CBR-state vector are either 0 or 2 , indicating that none of the nodes that have not yet transmitted have successfully received the message. Such states are marked in red, and hereafter this condition is referred to as a $C B R$ outage. Each transient state in the Markov process corresponds to a single CBR state, and such states are numbered 1 through 6 in the diagram. The 4 CBR states that correspond to a CBR outage are collapsed into a single absorbing state of the Markov process (state 7 ), and similarly the 9 CBR states corresponding to a CBR success are collapsed into a single absorbing state of the Markov process (state 8).

Consider how the CBR state may change from time $t$ to time $t+1$. All nodes at time $t$ with node state 1 transmit, so at time $t+1$, those nodes will now be in state 2 . All nodes that have node state 0 at time $t$ will receive the transmission, so at time $t+1$ these nodes will be either in state 1 (if the transmission was successful) or 0 (if the transmission failed). Since the channels from transmitting to receiving nodes are independent, the state transition probability is the product of the individual transmission probabilities. For instance, the probability of going from state [1100] to [2210] is the product of the probability that $N_{2}$ successfully decodes the joint transmission (from $S$ and $N_{1}$ ) and the probability that $D$ does not successfully decode the transmission. The individual probability of successful decoding at each node is found using the outage probability evaluated by (4.9). While there is a one-to-one correspondence between $\mathrm{CBR}$ states and the transient Markov states, there are several CBR states associated with each of the two absorbing Markov states. Thus, for each absorbing Markov state, the probability of transitioning into it is equal to the sum of the probabilities of transitioning into the constituent CBR states.

The Markov transition probabilities are placed as in $(9.2)$, and the two absorbing states are indexed so that the first absorbing state corresponds to a CBR outage, while the second 


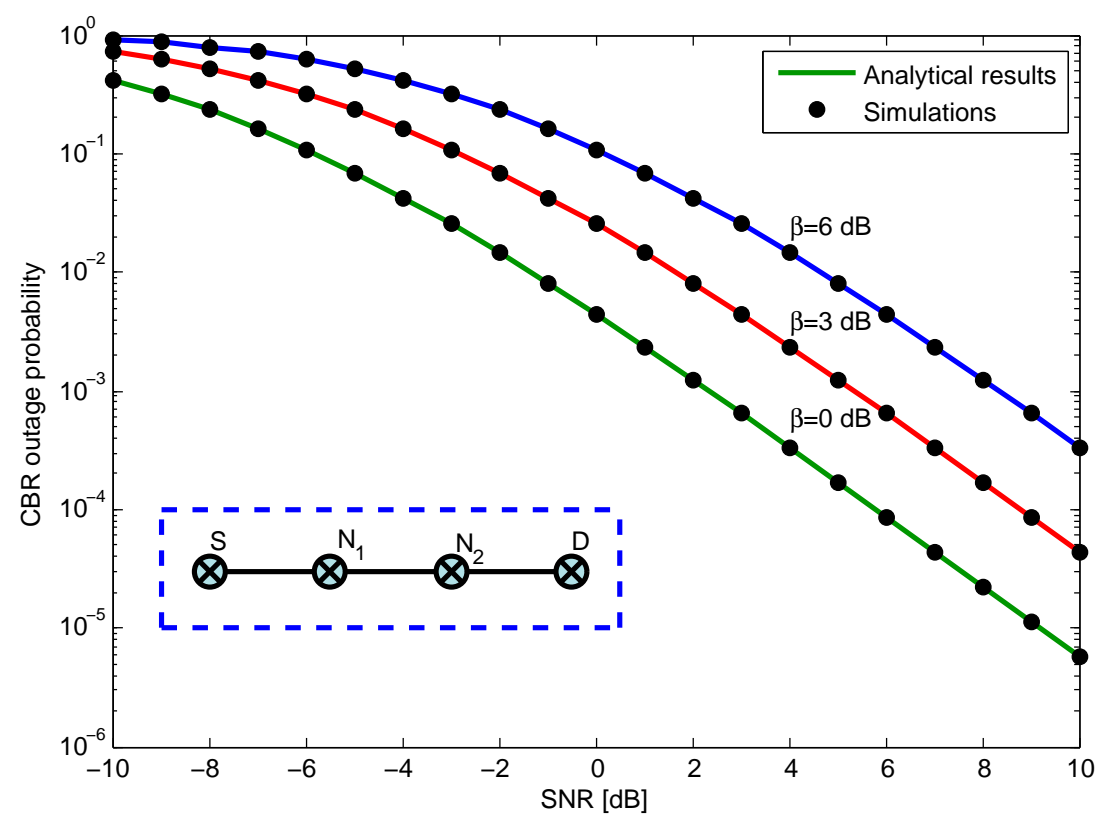

Figure 9.3: CBR outage probability as a function of the SNR when CCI is neglected. The solid lines are obtained analytically while the markers are obtained from Monte Carlo simulation. The network topology is a four node line network shown in the inset, and $\alpha=3.5$.

corresponds to a $\mathrm{CBR}$ success. By using $(9.3)$, and since the process always starts in state $s_{1}$, it follows that $b_{1,1}$ is the $C B R$ outage probability (which is indicated by $\epsilon_{C B R}$ ) and $b_{1,2}$ is the $C B R$ success probability (which is $\hat{\epsilon}_{C B R}=1-\epsilon_{C B R}$ ).

As an example consider the four-node CBR whose nodes are equally spaced along a line, as shown in the inset in Fig. 9.3. In this example, both the intra-acCBR and inter-CBR intereference is neglected, and the path loss exponent is $\alpha=3.5$. Assume Rayleigh fading $\left(m_{i, j}=1\right.$ for all $i$ and $\left.j\right)$. Fig. 9.3 shows the CBR butage probability as a function of the SNR for three values of the threshold $\beta$. Solid curves are obtained analytically according to the methodology described in this chapter, while the markers correspond to a simulation using the methodology introduced in Chap. 8. In particular, the simulation works by first computing the outage probabilities using (4.9), using these probabilities to determine the state-transition probabilities, and simulating each state transition by drawing a random number. During each Monte Carlo trial, the set of links that provide successful transmission is identified and if so there exists a set of links that connects the source to the destination. If there is no set of links that connects the source to the destination, a routing failure is counted. The analytical results coincide with the simulations as shown in Fig. 9.3, and any 
discrepancy between the curves can be attributed to the finite number of Monte Carlo trials ( $10^{7}$ trials were executed per SNR point).

\subsubsection{Inter-CBR Interference}

This subsection focuses only on inter-CBR interference and assumes that the transmission from a $\overline{C B R}$ s source to its destination must take place within one radio frame or else an outage occurs. Since each transmission requires one slot, the maximum number of slots per radio frame is also $N+1$. While variable-length frames could be supported, this would complicate the synchronization of neighboring CBRs. It is thus assumed that all $\overline{C B R} s$ have $N$ interior nodes and $F=N+1$.

\section{Iterative method}

Let $\mathbf{P}_{k}$ represent the transition matrix of the $k^{\text {th }}$ CBR. The transition probabilities in $\mathbf{P}_{k}$

are computed using 4.9, which depend on the $\left\{p_{i}^{(t)}\right\}$ associated with nodes in other CBR. However, each $p_{i}^{(t)}$ represents the probability that node $X_{i}$ transmits during time slot $t$, which can be found from the $\mathbf{P}_{k}$ of the corresponding CBR. Thus, interference causes a linkage between the transition probabilities in the given $\mathrm{CBR}$ and the transmission probabilities of adjacent CBR;. While this linkage could be handled by considering the overall BRN as one large Markov chain, this is a cumbersome solution as the number of states grows exponentially with the number of nodes. A more efficient approach is an iterative one, which alternates between computing the $\left\{p_{i}^{(t)}\right\}$ and the $\left\{\mathbf{P}_{k}\right\}$.

Let $\mathcal{S}_{i, t}$ be the set of states associated with time slot $t$ and labeled with a ' 1 ' in the position corresponding to $X_{i}$. These are the states for which $X_{i}$ transmits during time slot $t$. The probability $p_{i}^{(t)}$ can be found by adding the probabilities of being in each of the states in $\mathcal{S}_{i, t}$, given that the system starts from initial state $\mathbf{s}_{1}$. The probability of being in state $\mathbf{s}_{j} \in \mathcal{S}_{i, t}$ is found by multiplying the probabilities of all transitions in the Markov chain leading from state $s_{1}$ to state $s_{j}$

Let $\boldsymbol{P}_{k}\left[i_{t}\right]$ represent the state transition matrix for the $k^{\text {th }} \mathrm{CBR}$ corresponding to iteration $i_{t}$, and similarly let $p_{i}^{(t)}\left[i_{t}\right]$ represent the transmission probability for the $i^{t h}$ user during the 


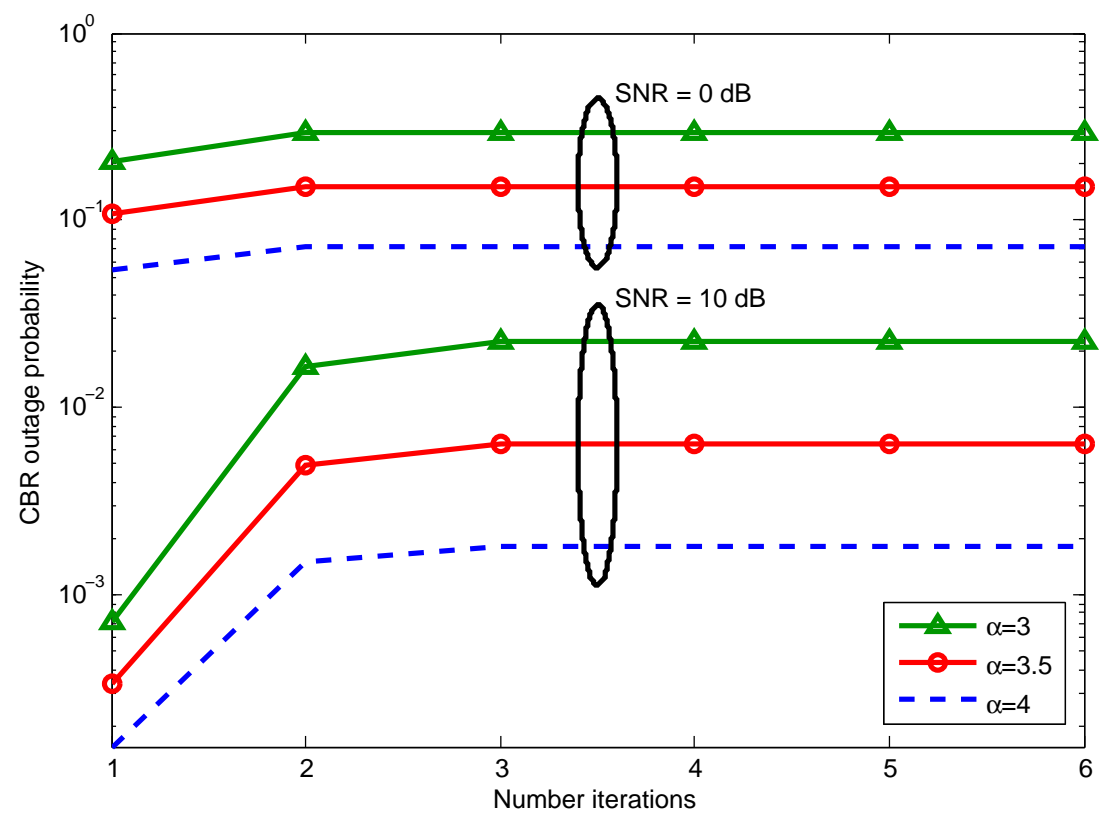

Figure 9.4: CBR outage probability for the $k^{\text {th }} \mathrm{CBR}$ as function of the number of iterations used, when only the inter-CBR interference is considered. Set of curves at the top: SNR $=0$ $\mathrm{dB}$. Set of curves at the bottom: SNR $=10 \mathrm{~dB}$. Each set of curves is obtained using three values of $\alpha$.

$t^{t h}$ time slot corresponding to iteration $i_{t}$. The iterative method can be described as follows:

1. Initialization: Set $i_{t}=0$ and initialize $p_{i}^{(t)}[0]=0, \forall i, t$, which corresponds to neglecting interference. Then compute each $\boldsymbol{P}_{k}[0]$ using the initial $\left\{p_{i}^{(t)}[0]\right\}$.

2. Recursion: Increment $i_{t}$. Evaluate each $\boldsymbol{P}_{k}\left[i_{t}\right]$ using the $\left\{p_{i}^{(t)}\left[i_{t}-1\right]\right\}$ from the previous iteration.

3. Decision: Halt the process if $\left\|\left(\boldsymbol{P}_{k}\left[i_{t}\right]-\boldsymbol{P}_{k}\left[i_{t}-1\right]\right)\right\|_{F}<\xi, \forall k$, where the operator $\|\cdot\|_{F}$ is the Frobenius norm and $\xi$ is a tolerance. Otherwise, go back to step 2.

The approach is further simplified if it is assumed that the BRN is comprised of an infinite cascade of $\overline{\mathrm{CBR}}$; , all having identical topology. Otherwise, each $\mathrm{CBR}$ can be modeled by a different Markov chain characterized by a specific $\mathbf{P}_{k}$. Under the assumption of an infinite cascade of identical $\overline{\mathrm{CBR}} \boldsymbol{s}$, the $\mathbf{P}_{k}$ and the set of $\left\{p_{i}^{(t)}\right\}$ will be the same in all active zones. Because of this, performance can be described by a typical CBR.

As an illustrative example, assume that each CBR is composed of four nodes which are configured as in the inset of Fig. 9.3. The typical CBR is analyzed assuming it receives 
CCI from just the two closest adjacent active zones (the interference from distant zones is neglected). Fig. 9.4 shows the CBR outage probability for a typical CBR as a function of the number of iterations used in the algorithm described above. Curves are shown for two values of SNR and three values of $\alpha$. All six curves are obtained with $\beta=6 \mathrm{~dB}, \alpha=3.5$ and under the assumption of Rayleigh faded channels. Fig. 9.4 shows that the CBR outage probability increases as a function of the number of iterations, and that the iterative method converges rapidly after very few iterations.

\section{Network Optimization}

In the context of a BRN, the ASE for a line network is

$$
\mathcal{S}_{\text {ase }}=T d_{\mathrm{CBR}}
$$

where $T$ is the throughput of the $\overline{\mathrm{CBR}}$ and $d_{\mathrm{CBR}}$ is the distance between the $\overline{\mathrm{CBR}} \mathrm{s}$ source and destination. From [?], the throughput can be written as

$$
T=\frac{\left(1-\epsilon_{C B R}\right)}{F} R
$$

where $R$ is the code rate expressed in units of bits per channel use (bpcu).

Substituting (9.6) into (9.5), and relating the rate and the SINR threshold the Shannon capacity for complex discrete-time AWGN channels, yields

$$
\mathcal{S}_{\text {ase }}=d_{\mathrm{CBR}} \frac{\hat{\epsilon}_{C B R}}{2(N+1)} \log _{2}(1+\beta)
$$

where the factor 2 in the denominator is a consequence of the buffer nodes operating in half-duplex mode, or, equivalently, due to alternating between active and silent zones

Let $\boldsymbol{X}_{k}$ be a vector containing the position of the $N$ relays in the $k^{t h}$ CBR. The goal of the network optimization is to determine the set $\Theta=\left\{\boldsymbol{X}_{k}, R, N, d_{\mathrm{CBR}}\right\}$ that maximizes (9.7). Because (9.7) is nonlinear in $\Theta$ and the search space is multi-dimensional, efficiently finding a solution is a challenge. However, by initially using an exhaustive search, we have confirmed that the optimization surface is convex over the set $\left\{R, N, d_{\mathrm{CBR}}\right\}$. Thus, the optimization can be solved through a convex optimization over $\left\{R, N, d_{\mathrm{CBR}}\right\}$ combined by a stochastic optimization over $\boldsymbol{X}_{k}$. It follows that an efficient approach to find the optimal set $\boldsymbol{\Theta}$ is as follows: 
1. For each of the parameters $N, R$ and $d_{\mathrm{CBR}}$, select a pair of endpoints and a midpoint $\left(3^{3}=27\right.$ points $)$. The endpoints should initially be far enough apart to guarantee that the optimal point lies within the endpoints.

2. Define a reference vector $\boldsymbol{X}_{\text {ref }}\left(N, d_{\mathrm{CBR}}\right)$, which is a vector $\boldsymbol{X}_{k}$ for the pair $\left(N, d_{\mathrm{CBR}}\right)$ and a reference set $\left\{\boldsymbol{X}_{\text {ref }}\left(N, d_{\mathrm{CBR}}\right)\right\}$ containing the reference vectors of all $\left(N, d_{\mathrm{CBR}}\right)$ considered by the algorithm. Create three vectors $\left\{\boldsymbol{X}_{k}\right\}$ for each pair of $\left(N, d_{\mathrm{CBR}}\right)$. When $\left(N, d_{\mathrm{CBR}}\right)$ is chosen for the first time, the first vector of $\left\{\boldsymbol{X}_{k}\right\}$ is obtained by placing the $N$ relays on the length- $d_{\mathrm{CBR}}$ line connecting the source and the destination and this vector is added to the reference set, constituting $\boldsymbol{X}_{\text {ref }}\left(N, d_{\mathrm{CBR}}\right)$ for the pair $\left(N, d_{\mathrm{CBR}}\right)$ chosen. The placement is done in such way that relays are separated by a minimum distance $r_{\mathrm{s}}=\frac{d_{\mathrm{CBR}}}{3 N}$, but are otherwise uniformly distributed along the line. If the pair $\left(N, d_{\mathrm{CBR}}\right)$ has been already used, the first vector of $\left\{\boldsymbol{X}_{k}\right\}$ is set to be equal to $\boldsymbol{X}_{\text {ref }}\left(N, d_{\mathrm{CBR}}\right)$. The other two vectors are obtained by mutating $\boldsymbol{X}_{\text {ref }}\left(N, d_{\mathrm{CBR}}\right)$ as follows:

(a) For each relay in $\boldsymbol{X}_{\text {ref }}\left(N, d_{\mathrm{CBR}}\right)$, draw a Bernoulli random variable indicating if the relay should be moved or if it should remain in its current location.

(b) If the node $X_{i}$ is to be moved, the direction (right or left) of movement is selected with equal probability. The new position is obtained by moving the current position in the selected direction by a distance $\Delta=\frac{d_{\mathrm{CBR}}}{n_{\Delta} N}$, where initially $n_{\Delta}=2$.

3. Compute the ASE by (9.7) for the endpoints and the midpoints of the interval of one of the parameters in the set $\{R, N, d\}$ and for the different sets of locations of the relays. For each pair $(N, d)$ chosen, determine the vector $\boldsymbol{X}_{k}$ that provides the higher ASE and replace the $\boldsymbol{X}_{\text {ref }}(N, d)$ in the reference set with this vector.

4. For the same parameter of the set $\left\{R, N, d_{\mathrm{CBR}}\right\}$ used in step 3 , move the midpoint of the interval towards the endpoint that gives higher ASE, For each pair $\left(N, d_{\mathrm{CBR}}\right)$, generate the three sets of positions as in step 2.

5. Repeat step 3 and 4 recursively for all the parameters in the set $\left\{R, N, d_{\mathrm{CBR}}\right\}$ and 
Table 9.1: Optimization results when inter-CBR interference is considered.

\begin{tabular}{|c|c|c|c|c|c|c|}
\hline SNR (in $\mathrm{dB})$ & $\mathrm{CCI}$ & $R$ & $N$ & $d_{\mathrm{CBR}}$ & Optimal nodes' location for a line network & $\mathcal{S}_{\text {opt }}$ \\
\hline \multirow{3}{*}{0} & \multirow{3}{*}{$x$} & & & & s & \\
\hline & & 4.452 & 0 & 0.3 & & 0.490 \\
\hline & & 4.421 & 5 & 1.2 & 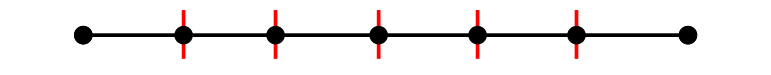 & 0.402 \\
\hline \multirow{3}{*}{5} & \multirow[b]{3}{*}{$\checkmark$} & & & & s & \\
\hline & & 4.611 & 0 & 0.4 & $\bullet$ & 0.683 \\
\hline & & 4.452 & 5 & 1.7 & $S \quad c^{S}$ & 0.559 \\
\hline \multirow[t]{2}{*}{10} & \multirow[t]{2}{*}{$x$} & 5.028 & 0 & 0.5 & s & 0.951 \\
\hline & & 4.547 & 5 & 2.3 & $S \quad \bullet^{S}$ & 0.777 \\
\hline
\end{tabular}

gradually reduce for one parameter at the time the range between the endpoints and the midpoint until a certain tolerance is reached. If the optimal ASE remains the same as in the previous iteration $n_{\Delta}$ is increased by one, until a certain value is achieved.

6. If the optimal ASE remains the same as in the previous iteration, for all the parameters in the set $\left\{R, N, d_{\mathrm{CBR}}\right\}$ it is not possible to further reduce the range between the endpoints and the midpoint, since a given tolerance is achieved, and $n_{\Delta}$ reaches its maximum value, the algorithm stops.

Using the methodology described above, optimization results were obtained under the same assumptions that were used in the previous subsection. In particular, it is assumed that the $\mathrm{BRN}$ extends infinitely and $\mathrm{CBR} s$ are composed of line networks with the same number of relays which are all equally positioned. For each CBR the only CCI considered are the ones from the two closest adjacent active zones (the interference from farther active zones is neglected, since they are at least at $3 d_{\mathrm{CBR}}$ distance away). Although these assumptions can be relaxed using the analysis and methodology proposed along this chapter, in this subsection they are used for simplicity of exposure.

Table 9.1 shows the optimal values of $N, R, d_{\mathrm{CBR}}$ and the optimal location for the $N$ relays. Optimization results are provided for three values of $\Gamma$ and when the $C \mathrm{CC}$ is neglected 
as well as when they are taken into account. Vertical red lines indicate the optimal position of the mobiles for SNR $=0 \mathrm{~dB}$, and they are used to facilitate the graphical analysis and comparison of the optimal location of the mobiles for different scenarios. For all scenarios considered, the path loss exponent is fixed to $\alpha=3.5$. Table 9.1 emphasizes that when there is no CCI, the optimal configuration for a cooperative $\mathrm{BRN}$ is to use short $\mathrm{CBR}$; characterized by a point-to-point conventional communication, while five relays distributed over a larger CBR allow to achieve the optimal $\mathrm{ASE} \mathcal{S}_{\text {opt }}$ when there is CCI from the adjacent CBRs. As expected, the optimal ASE increases by increasing SNR, since the links experience a more favorable channel. Furthermore, the optimal ASE increases going from a scenario in which there is CCI to one in which CCI is neglected, and this is more prominent as SNR increases since the network becomes more interference sensitive. In agreement with [154], an increase in SNR produces a shift towards the source of the optimal position of the relays, which increases the likelihood to cooperate among themselves. A more favorable condition of the channel produces an increment in both the optimal code rate $R$ and in the optimal dimension of the CBR,

\subsubsection{Intra-CBR Interference}

While in the previous subsection, it is assumed that a time frame is sufficiently long to ensure that a packet reaches the destination before a new packet is injected within a CBR, this is not generally valid. In fact, in practice BRN; often employ a fixed frame length of $F=4$ and a fixed maximum number of relays that is greater than $F[150]$. In such networks, spatial pipelining enables multiple packets, which will share the same radio resources, to be active simultaneously within a given CBR. As previously mentioned, a new packet is injected in a given CBR during the first time slot of each new radio frame, and when multiple packets propagate with a given CBR, they interfere with each other. In order to account for this source of interference within the aforementioned framework and in order to account for the effect of correlation among packets an iterative method is introduced, which is based on a Viterbi-like algorithm. Finally, similar to the previous subsection, an optimization is performed for a line network subject to intra-CBR interference in order to 
maximize its throughput by opportunely selecting the rate, the number of relays of which a $\mathrm{CBR}$ is composed, as well as the number of time slots that compose the radio frame.

\section{Iterative method}

Let $\mathbf{P}_{k}^{z}$ represent the transition matrix of the $k^{t h}$ CBR for the $z^{t h}$ packet. When the interCBR interference is neglected, but multiple packets can simultaneously propagate within the same CBR, there is a linkage between the transition probabilities for the $z^{\text {th }}$ packet $\mathbf{P}_{k}^{z}$ and the transmission probabilities of the nodes within the $k^{\text {th }} \mathrm{CBR}$ that may be active in letting other packets propagate during the interval of time the $z^{\text {th }}$ packet is broadcasted. Furthermore, the probabilities of transmission for the $z^{\text {th }}$ packet during a given radio frame depends also on the reciprocal interference produces by packets that have been propagating or had propagated within the $k^{\text {th }}$ CBR since the very first transmission. These linkages could be handled by considering a large Markov chain, which describes the full dynamics in time of the $k^{t h}$ CBR. However, this solution is in most cases computational infeasible. A more appropriate approach is to use a Viterbi-like algorithm to iteratively update the transition matrix of the $k^{\text {th }} \mathrm{CBR}$ on a radio frame basis, alternating between the computation of the $\left\{\mathbf{P}_{k}^{z}\right\}$ and the $\left\{p_{i, z}^{(t)}\right\}$, where $p_{i, z}^{(t)}$ is the probability that $i^{\text {th }}$ node transmits the $z^{\text {th }}$ packet during time slot $t$.

Let $\mathcal{Z}[f]$ indicate the set of packets that might be active during the $f^{\text {th }}$ radio frame, which are ordered based on when they are first injected. Let $\mathcal{Z}[f](i)$ be the $i^{\text {th }}$ element of the set $\mathcal{Z}[f]$ and let $N_{p k}[f]=|\mathcal{Z}[f]|$ is their number. Let $\boldsymbol{P}_{k}^{z}\left[i_{t}\right]$ represent the state transition matrix for the $k^{\text {th }}$ CBR at iteration $i_{t}$ for the $z^{\text {th }}$ packet, when it is active during the $f^{\text {th }}$ radio frame, and similarly let $p_{i, z}^{(t)}\left[i_{t}\right]$ represent the transmission probability at iteration $i_{t}$ for the $i^{\text {th }}$ user when it transmits the $z^{\text {th }}$ packet during the $t^{t h}$ time slot of the $f^{\text {th }}$ radio frame. Let $\mathcal{I}_{f}$ indicate the maximum number of iterations needed to evaluate the transition matrix for the packet that is first transmitted during the $f^{\text {th }}$ radio frame. The iterative method can be described as follows:

1. Initialization: Set $f=1$ and $i_{t}=0$. Find the set $\mathcal{Z}[1]$ of packets that might be active during the first radio frame. Initialize $p_{i, z}^{(t)}[0]=0, \forall i, t$ and $z \in \mathcal{Z}[1]$. Note, that this 
corresponds to neglecting interference within the $k^{\text {th }}$ CBR, during the duration of the first radio frame. Furthermore, it allows the iterative method to start from the scenario where the first packet injected within the $k^{\text {th }}$ CBR is not subject to any intra-CBR interference, unless it is still propagating during one of the successive radio frames. Compute $\boldsymbol{P}_{k}^{z}[0]$ using the initial $\left\{p_{i, z}^{(t)}[0]\right\}$ for all $z \in \mathcal{Z}[1]$.

2. Recursion:

(a) Increment $i_{t}$. Compute $\boldsymbol{P}_{k}^{z}\left[i_{t}\right]$ using the $\left\{p_{i, z}^{(t)}\left[i_{t}\right]\right\}$ from the previous iteration for all $z \in \mathcal{Z}[f]$.

(b) Go back to step $2 \mathrm{a}$ until $\left\|\left(\boldsymbol{P}_{k}^{z}\left[i_{t}\right]-\boldsymbol{P}_{k}^{z}\left[i_{t}-1\right]\right)\right\|_{F}<\xi, \forall z \in \mathcal{Z}[f]$, where the operator $\|\cdot\|_{F}$ is the Frobenius norm and $\xi$ is a tolerance.

3. Decision: Halt the process if both the following conditions are met:

- $\left\|\left(\boldsymbol{P}_{k}^{\mathcal{Z}[f](i)}\left[\mathcal{I}_{f}\right]-\boldsymbol{P}_{k}^{\mathcal{Z}[f-1](i)}\left[\mathcal{I}_{f-1}\right]\right)\right\|_{F}<\xi, \forall i \in\left\{1, \cdots, N_{p k}[f-1]\right\}$;

- $f \neq 1$.

Otherwise,

(a) Increment $f$. Set $i_{t}=0$. Evaluate the set $\mathcal{Z}[f]$ of packets that might be active during the $f^{\text {th }}$ radio frame.

(b) go back to step 2 .

Note that this approach not only accounts for the reciprocal interference that different packets produce to each other, but also for the effect of the correlation among them. As an illustrative example, assume that a $\mathrm{CBR}$ is composed of four nodes $(N=2)$ which are configured again as in the inset of Fig. 9.3 . The CBR is analyzed by neglecting the interCBR interference. Fig. 9.5 shows the CBR outage probability when the length of the radio frame is varied within the set $F=\{1,2\}$ as function of the number of radio frames that need to be considered in the aforementioned iterative method. Curves are shown for three values of the path loss exponent. All curves are obtained with $\beta=6 \mathrm{~dB}, \mathrm{SNR}=0 \mathrm{~dB}$, and under the assumption of Rayleigh faded channels. Fig. 9.5 shows that the iterative method converges very quickly. 


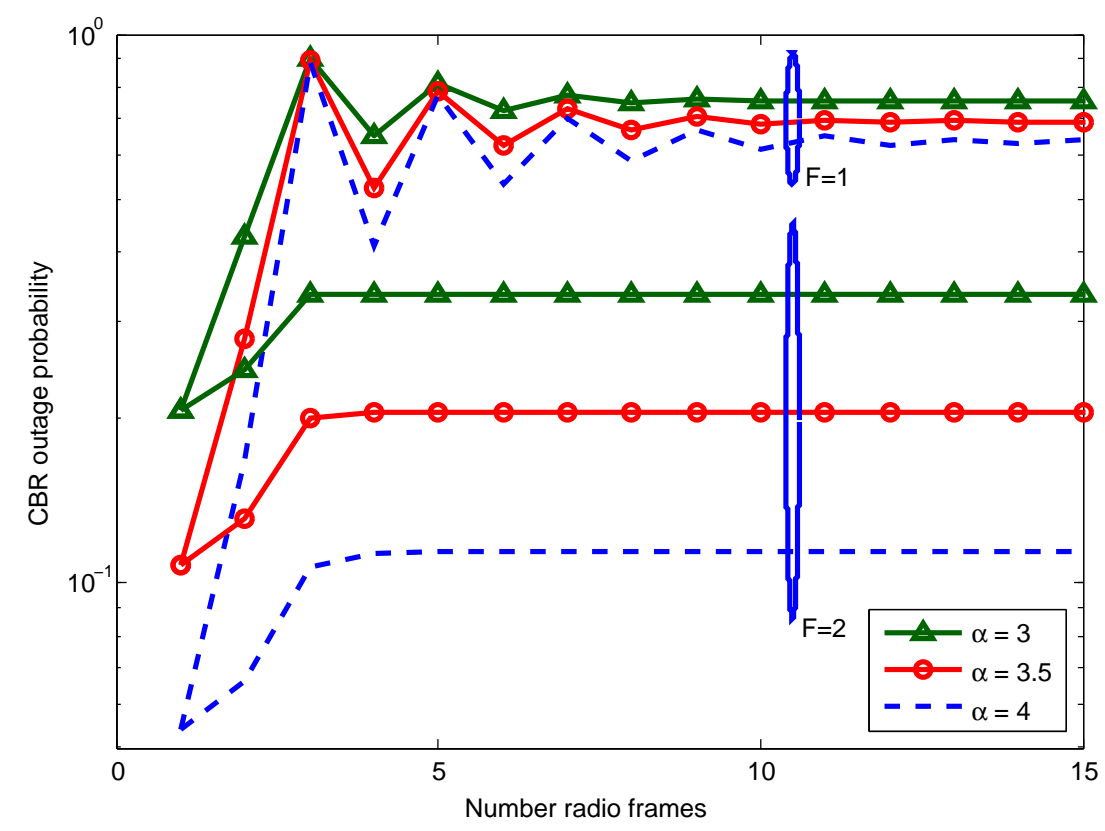

Figure 9.5: CBR outage probability for the $k^{\text {th }}$ CBR as function of the number of radio frames considered, when only the intra-CBR interference is taken into account.

\section{Network Optimization}

Using (9.5) and (9.6), and by relating again the rate and the SINR threshold by the Shannon capacity for complex discrete-time AWGN channels, in this scenario the ASE can be written as follows

$$
\mathcal{S}_{\text {ase }}=d_{\mathrm{CBR}} \frac{\hat{\epsilon}_{C B R}}{F} \log _{2}(1+\beta)
$$

As can be observed in (9.8), there is a tradeoff between the rate, the number of relays that compose a CBR, and the number of time slots in a radio frame. Therefore, the goal of the network optimization is to determine the set $\Theta=\{R, N, F\}$ that maximizes (9.8). While an exhaustive search could be a good alternative to this type of non-linear optimization problem, a more efficient method can be used. In particular, after finding that the optimization surface is convex over $\{R, N, F\}$, a more efficient approach to find the optimal set $\Theta$ is as follows:

1. For each of the parameters $N, R$ and $F$, select a pair of endpoints and a midpoint $\left(3^{3}=27\right.$ points $)$. The endpoints should initially be far enough apart to guarantee that the optimal point lies within the endpoints. 
Table 9.2: Optimization results when intra-CBR interference is considered.

\begin{tabular}{|c||c||c||c||c||c|}
\hline$d_{\mathrm{CBR}}$ & SNR (in dB) & $R$ & $N$ & $F$ & $\mathcal{S}_{\text {opt }}$ \\
\hline \hline \multirow{2}{*}{1} & 0 & 4.389 & 7 & 3 & 1.361 \\
\cline { 2 - 5 } & 5 & 5.676 & 7 & 3 & 1.811 \\
\cline { 2 - 5 } & 10 & 5.028 & 6 & 2 & 2.509 \\
\hline \hline \multirow{2}{*}{0.5} & 0 & 5.028 & 5 & 2 & 1.256 \\
\cline { 2 - 5 } & 5 & 6.658 & 5 & 2 & 1.6615 \\
\cline { 2 - 6 } & 10 & 8.309 & 5 & 2 & 2.062 \\
\hline
\end{tabular}

2. Compute the ASE by (9.8) for the endpoints and the midpoints of the interval of one of the parameters in the set $\{R, N, F\}$.

3. For the same parameter of the set $\{R, N, F\}$ used in step 2 , move the midpoint of the interval towards the endpoint that gives higher ASE,

4. Repeat step 2 and 3 recursively for all the parameters in the set $\{R, N, F\}$ and gradually reduce for one parameter at the time the range between the endpoints and the midpoint until a certain tolerance is reached. If the optimal ASE remains the same as in the previous iteration, the algorithm stops.

Using the methodology described above, optimization results were obtained under the assumption that the inter-CBR interference can be neglected ${ }^{3}$. A line network is considered, which is composed of a CBR with nodes disposed at equal distance from each other.

Table 9.2 shows the optimal values of $N, R$, and $F$. Optimization results are provided for three values of $\Gamma$. Two length for the CBR are considered. For all the scenarios considered, the path loss is fixed to $\alpha=3.5$. Table 9.2 shows that as expected the optimal ASE increases by increasing SNR. More importantly, as the SNR increases, it is possible to more frequently inject new packets by reducing the length of the radio frame and slightly reduce the code rate. As the length of the $\mathrm{CBR}$ is reduced, a fewer number of relays is required, and while as expected the throughput increases, a lower ASE is achieved.

\footnotetext{
${ }^{3}$ Note that here for sake of simplicity the inter-CBR interference is neglected, but it can be taken into account by jointly using the iterative method described in this subsection, together with the one described in the previous subsection.
} 


\subsection{Chapter Summary}

This chapter has presented a novel analytical framework to model and analyze cooperative multi-hop networks. The main advantage of this framework compared to those in the literature is in the reduced computational effort required, since it builds on the analysis developed along this dissertation, which allows to evaluate the link outage probability for diversity combining in closed-form and doesn't require any averaging over the fading gains. This framework is then applied to analyze and optimize unicast in a BRN. A BRN is analyzed by describing the behavior of each constituent CBR; as a Markov chain and the transition probabilities are computed by using a closed form expression for the outage probability, which takes into account the path loss, Rayleigh fading, and interference. Both inter-CBR and intra-CBR interference are considered. Both sources of interference cause an interdependence between the transmission and transition probabilities, which is taken into account by an iterative method. In the case of inter-CBR interference, the iterative method updates the probability of collisions for each CBR at each iteration for each one of the time slots. In contrast, intra-CBR is handled by using a Viterbi-like algorithm to iteratively update the transition probabilities on a radio frame basis, while still accounting for the interdependence between packets that simultaneously propagate within a $\mathrm{CBR}$ and their temporal correlation. The analysis and each iterative method have been used to perform an optimization of a $\overline{B R N}$ by accounting individually for a single type of interference. First only the inter-CBR interference are considered, and the aim has been to maximize the ASE by finding the optimal number of relays that need to be used in the CBRs that compose the BRN, their optimal placement, the optimal size of the $\overline{C B R}$; and the optimal code rate at which the nodes transmit. Finally, the effect of intra-CBR interference is studied and the network is optimized for several different scenarios with the aim again of maximizing the ASE by choosing opportunely the length of a radio frame, the code rate at which a node transmits and the number of relays involved in the communication. While the analysis and the model have been applied to study and optimize a BRN, this work could be extended to different types of cooperative ad hoc networks. 


\section{Chapter 10}

\section{Conclusion}

In this dissertation, the effect of interference and computational complexity has been studied in the context of modern wireless communication systems. The first main contribution of this dissertation is the development of a novel analytical framework that captures the effects of interference and fading by evaluating the outage probability for wireless networks with interferers located according to a point process when the signal is subject to fading. This framework is applied to a variety of cases in order to demonstrate its wide applicability and benefits in modeling both conventional and cooperative networks (i.e., conventional and cooperative cellular networks, and multi-hop ad hoc networks).

The second main contribution of the dissertation is the development of an analytical framework to estimate the computational resources required to decode a signal under a given performance constraint. This framework has been used to highlight the benefit of pooling computational resources and centrally processing uplink signals as an alternative to a conventional distributed approach. A better understanding of the interplay between available computing resources and the required communications performance is provided. The dissertation developed computationally aware schedulers, in order to better manage the resources available in systems such as Cloud-RAN; , where the quality of communications is strongly effected by the limited computational resources available for processing the communication signals.

By leveraging its proposed analytical framework, the dissertation provided a novel methodology to optimize future-generation multiple-access networks, including $[\mathrm{FH}$ systems, with the 
aim to further mitigate the effect of frequency-selective fading and prevent the near-far problem. The optimization accounts for the modulation format used, but also for the presence of fading and interference effects, including $\mathrm{CCI}$ and $\mathrm{ACT}$. This work has concluded by describing a more efficient and accurate method to stochastically model and study the performance of cooperative multi-hop wireless networks in presence of interference, which was applied to model and optimize a BRN,

\subsection{Summary and Publications}

After an introductory chapter, the discussion starts in Chap. 2, which builds an alterative analytical framework to evaluate the outage probability of an arbitrarily shaped network subject to fading and with the location of the interferers distributed according to a point process. In this chapter, the effect of spatial correlation of the interference has been also quantified. The main contribution of this chapter lies in the fact that the common assumption of an infinite network has been relaxed, and the analysis is broken into multiple steps, which help to distinguish the effect of fading, shadowing and spatial distribution, which are mechanisms that operate over different time scales. A closed-form expression is provided for the outage probability averaged over the fading and conditioned on the topology of the network and shadowing gains. This expression is particularly useful and results to be very efficient when analytical untractable spatial models are adopted, or more sophisticated shadowing models or path loss models want to be considered. In this case, the use of simulations is necessary, but they are notably simplified since the effect of the small scale fading is already taken into account. The analytical framework described in this chapter has been initially published and presented at the 2012 Virginia Tech Symposium on Wireless Personal Communication |1 under the simplified assumption of Rayleigh fading and a circular network, and then in IEEE Communication Letters $[2]$ for an arbitrarily shaped network. The work on the quantification of the spatial correlation has been instead enclosed in a manuscript under preparation [3].

In order to prove the wide applicability of the framework introduced in Chap. 2, Chap. 3 applies it to model and analyze the performance of DS-CDMA cellular uplinks. The model and the analysis, are very general and can handle a very large variety of MAC protocols 
and resource allocation policies. The main contribution of this chapter lies in the adoption of a more accurate spatial model for both the $\overline{\mathrm{BS}}$; and $\overline{\mathrm{UE}}$, which guarantees a minimum separation among devices and mimics more closely the deployment of an actual cellular network. Furthermore, the analysis allows to discharge the common assumptions of identical fading conditions for each link and a distance dependent path loss model with a single slope. The results contained in this chapter have been initially published and presented at the 2013 IEEE International Conference on Computing, Networking, and Communications [4] for the downlink, and at the 2013 IEEE International Conference on Communication [5] for the uplink. The work leading to this chapter has been also published on IEEE Transaction on Communications [6].

The analytical framework built in Chap. 2 has been extended in Chap. 4 in order to account for the case when a receiving device performs diversity combining of signals from multiple source transmitters. In this case, the analysis is applied to model and analyze a DS-CDMA downlink MCC and a MBSFN, The main contribution lies in the derivation of a closed-form expression for the outage probability averaged over the fading, which allow to adopt a more accurate spatial model, a distance dependent fading model and doesn't preclude the usage of a path loss model with multiple slopes. This work has been published and presented at the 2013 IEEE Global Telecommunication Conference [7] and at the 2014 IEEE International Conference on Acoustic, Speech and Signal Processing [8].

Chap. 5 builts an analytical framework to estimate the required data processing resources under a given performance constraint, which allows to underline the tradeoff between the throughput and the computational complexity. In this chapter, the emphasis has been given to the Cloud-RAN architecture, and the developed analytical framework is used to highlight the benefits of pooling computational resources and centrally process uplink signals from a group of $\mathrm{BS}$ s also by the introduction of novel metrics ( i.e., computational outage, computational diversity and computational gain). As shown along Chap. 5, despite some simplificative assumptions that have been made and which are necessary for the tractability of the problem, the analytical framework is very accurate and provides a good match with simulations obtained through a system level simulator compliant with the 3GPP LTE standard. The analytical framework and the findings of this chapter have been published on 
IEEE Transaction on Wireless Communications [9].

Following from Chap. 5. Chap. 6 provides more insights on how the communications performance are effected by the computational resources available. In this chapter, three computational aware schedulers are designed for practical systems, which are able to significantly reduce the computational effort required at the receiver by opportunely selecting the MCS scheme to use for each uplink and improve the overall performance of the network by reducing or eliminating the likelihood of computational outages. The first scheduler called CAS chooses the MCS to adopt by opportunely backing-off from capacity with the aim to reduce the computational complexity required at the receiver in order to reduce the probability of computational outage. The other two schedulers, SWF and SCC, are designed with the aim to completely avoid computational outages while the objective is to maximize the achieved sum-rate. SWF is based on a water-filling algorithm, while $\mathrm{SCC}$ is more intuitive and simple and uses a complexity-cut-off approach. While the concepts of computational outage and detailed performance evaluation of CAS have been published and presented at the 2014 IEEE Global Telecommunication Conference [10], the remaining part of this chapter will be presented in the 2015 venue [11]. The work discussed in both Chap. 5 and Chap. 6 will also appear on a book chapter [12].

Chap. 7 focuses on $\mathrm{FH}$ systems in general, and in particular on ad hoc networks which uses a non-coherent CPFSK modulation. This modulation format depends on the modulation index, the number of frequency channels in which the overall bandwidth is divided and the fractional in-band power, which is the fraction of the signal power that lies within the band of each frequency channel. These parameters are often chosen arbitrarily. However, the chapter shows that under two different rate-allocation policies that optimally selecting them together with the rate, it is possible to achieve a significant improvement of the performance of the system. The results of this research have been published and presented at the 2012 IEEE Symposium on Military Communications [13], at the 2013 IEEE International Conference on Communication [14], and at the 2015 IEEE Symposium on Military Communications [15]. Furthermore, a manuscript, which will be submitted to IEEE Transaction on Communications [16 and summarizes all our results on the topic, is in preparation.

Chap. 8 introduces a new methodology to model and analyze the performance of multi- 
hop routing protocols in ad hoc networks which builds on the analytical framework introduced in Chap. 2. This chapter accounts for the effect of the interference and their spatial and temporal correlation and evaluates the performance of two representative geographic routing protocols in order to gain perspective about their advantages and disadvantages. The main contribution of this chapter is in proposed paradigm, which can be used for rapid performance assessment of a large variety of routing protocols. The methodology proposed in this chapter is able to discharge some of the common assumptions made in the related work in the litterature, such as the independence of the success probabilities of path from the source to the destination even when paths share the same links. This work has been published and presented at the 2013 and 2014 IEEE Symposium on Military Communications [17, 18 and has been accepted to publication on IEEE Transactions on Communications [19].

Chap. 9 introduces an efficient method to stochastically model and study the performance of cooperative multi-hop networks when accounting for CCI originated by multiple packets that simultaneously propagate along the network, their temporal correlation and interdependence. The dynamics of how a cooperative protocol evolves over time is described through an absorbing Markov chain, which uses the analysis developed in Chap. 4 to evaluate the transmission probability of each relay. This methodology differs from the ones existing in the literature, since the end-to-end probability of success of a cooperative network is not evaluated by starting from a single set of channel realizations (as it is usually done) and then averaging over them, but through the channel outage framework developed along this dissertation. As already stated, this framework provides the channel outage probability already averaged over the fading and in this context it drastically reduces the computational effort to model and analyze a cooperative multi-hop network. As an example of its applicability, this framework is used in this chapter to analyze the performance of a $B R N$ and perform an optimization of such a system over a set of parameters, which includes the code rate, and the number of relays used. The work related to inter-CBR interference has been published and presented at the 2014 IEEE Symposium on Military Communications [20], while the remaining work has been enclosed in a recently submitted manuscript [21]. 


\subsection{Future Work}

As already mentioned, the analytical frameworks proposed in this dissertation are very general and they can be adopted to several other applications and case of studies. Suggestions for future work and extensions of this work might include:

1. Cognitive Networks: The limited bandwidth available and the inefficient usage of the spectrum has led in the past years a significant amount of research devoted to cognitive radio. In a cognitive radio, a cognitive (secondary) user is allowed to share the spectrum with a primary users, as long as its interference is below a certain threshold. In this type of networks the geometry plays an important role and the analytical framework developed in Chap. 2 can be actually used to analyze the interaction between primary and secondary users.

2. Other diversity techniques: An attempt to expand the analytical framework developed in Chap. 2 for other diversity techniques can be made, which will open another large set of interesting applications and optimization problems.

3. MmWave and device-to-device communication: Since the analysis developed in Chap. 2 and Chap. 4 allows to consider non identical fading conditions at each link and a multiple slope path loss model can be adopted, the methodology introduced in Chap. 3 can be used to accurately model and analyze multi-antenna transmissions in millimiter-wave heterogeneous cellular networks and device-to-device communication.

4. Computational requirements: channel outage and interference: One of the weak points of the analytical framework developed in Chap. 5 is that the channel outage is not explicitly taken into account. In a real system, the computational outage and channel outage can both occur, in a non mutually exclusive manner, and they are both equally important for the overall performance of the network. For instance, when a channel outage occurs, the maximum computational complexity might be invested and the data processing requirements might change. Therefore another possible extension of this work might be to analytically account for the channel outage in the proposed framework, in order to estimate more accurately the data processing requirements of 
the network. Furthermore, the effect of co-channel interference needs to be taken into account, and bounds might be derived for the novel performance metrics introduced in this chapter. 


\section{Appendix A}

\section{Conditional Outage Probability: Derivation}

This appendix provides details leading to $\left[2.9\right.$. Let $\bar{F}_{\mathbf{Z}_{j}}(z \mid \Omega)=1-F_{\mathbf{Z}_{j}}(z \mid \Omega)$ denote the complementary $\mathrm{CDF}$ of $\mathbf{Z}_{\mathbf{j}}$ conditioned on $\boldsymbol{\Omega}$. Assuming that the Nakagami parameter $m_{0, j}$ of the source's channel is integer-valued, the CDF of $Z_{j}$ can be computed as follows 155:

$$
\bar{F}_{\mathrm{Z}_{\mathrm{j}}}(z \mid \Omega)=\mathbb{P}\left[\mathrm{Z}_{j}>z \mid \Omega\right]=\mathbb{P}\left[\mathrm{S}_{j}>z+\sum_{i}^{M} \mathrm{Y}_{i, j}\right]=\int \ldots \int f_{\mathbf{Y}}(\mathbf{y}) F_{\mathrm{S}_{j}}\left(z+\sum y_{i}\right) \mathrm{d} \mathbf{y}(\mathrm{A} .1)
$$

where the integral is over $M$-dimensional real space. Taking into account the Nakagami fading and the activity probability $p_{i}$, the $\overline{\mathrm{PDF}}$ of $f_{\mathbf{Y}}(\mathbf{y})$, which is the joint $\mathrm{PDF}$ of the vector $\mathbf{Y}=\left\{Y_{1, j}, \ldots, Y_{M, j}\right\}$, is

$$
f_{\mathrm{Y}_{i}}(y)=\left(1-p_{i}\right) \delta(y)+p_{i}\left(\frac{m_{i, j}}{\Omega_{i, j}}\right)^{m_{i, j}} \frac{y^{m_{i, j}-1}}{\Gamma\left(m_{i, j}\right)} \exp \left(-\frac{m_{i, j} y}{\Omega_{i, j}}\right) u(y)
$$

where $u(y)$ is the step function, $\delta(y)$ is the Dirac delta function, and

$$
\Gamma(x)=\int_{0}^{\infty} y^{x-1} e^{-y} \mathrm{~d} y .
$$

The gamma-distributed $\mathrm{S}_{j}$ with Nakagami parameter $m_{0, j}$ has a CDF, which is

$$
F_{\mathrm{S}_{j}}(s)=\exp \left(-\frac{\beta m_{0, j} s}{\Omega_{0, j}}\right) \sum_{\mu=0}^{m_{0, j}-1} \frac{1}{s !}\left[\frac{\beta m_{0, j} s}{\Omega_{0, j}}\right]^{s}
$$


Substituting (A.4) into A.1 and performing a few manipulations yields

$$
\begin{aligned}
\bar{F}_{\mathbf{Z}_{\mathbf{j}}}(z \mid \Omega)= & \exp \left(-\frac{\beta m_{0, j} z}{\Omega_{0, j}}\right) \sum_{\mu=0}^{m_{0, j}-1} \frac{\left(\beta m_{0, j} z\right)^{\mu}}{\Omega_{0, j}^{\mu} \mu !} \\
& \times \int_{\mathbb{R}^{M}} \ldots f_{\mathbf{Y}}(\mathbf{y}) \exp \left(-\frac{\beta m_{0, j}}{\Omega_{0, j}} \sum y_{i}\right)\left(1+z^{-1} \sum_{i=1}^{M} y_{i}\right)^{\mu} \mathrm{d} \mathbf{y} .
\end{aligned}
$$

Since $\mu$ is an integer, the binomial theorem gives the series expansion:

$$
\left(1+z^{-1} \sum_{i=1}^{M} y_{i}\right)^{\mu}=\sum_{t=0}^{\mu}\left(\begin{array}{l}
\mu \\
t
\end{array}\right) z^{-t}\left(\sum_{i=1}^{M} y_{i}\right)^{t}
$$

A multinomial expansion yields

$$
\left(\sum_{i=1}^{M} y_{i}\right)^{t}=t ! \sum_{\substack{\ell_{i} \geq 0 \\ \sum_{i=1}^{M} \ell_{i}=t}}\left(\prod_{i=1}^{M} \frac{y_{i}^{\ell_{i}}}{\ell_{i} !}\right)
$$

Substituting (A.6) and A.7) into (A.5), performing a few manipulations, and assuming that the interfering channels fade independently,

$$
\bar{F}_{\mathrm{Z}_{\mathrm{j}}}(z \mid \Omega)=\exp \left(-\frac{\beta m_{0, j} z}{\Omega_{0, j}}\right) \sum_{\mu=0}^{m_{0, j}-1} \frac{\left(\beta m_{0, j} z\right)^{\mu}}{\Omega_{0, j}^{\mu} \mu !} \sum_{t=0}^{\mu} \frac{z^{-t}}{(\mu-t) !} \sum_{\substack{\ell_{i} \geq 0 \\ \sum_{i=1}^{M} \ell_{i}=t}} \prod_{i=1}^{M} G_{\ell_{i}}\left(\Omega_{i, j}\right)
$$

where

$$
G_{\ell_{i}}\left(\Omega_{i, j}\right)=\int_{\mathbb{R}} \exp \left(-\frac{\beta m_{0, j} y}{\Omega_{0, j}}\right) \frac{y^{\ell_{i}}}{\ell_{i} !} f_{\mathrm{Y}_{i, j}}(y) \mathrm{d} y .
$$

Substituting A.2 in A.9,

$$
G_{\ell_{i}}\left(\Omega_{i, j}\right)=\left(1-p_{i}\right) \delta_{\ell_{i}}+\frac{p_{i} \Gamma\left(\ell_{i}+m_{i, j}\right)}{\ell_{i} ! \Gamma\left(m_{i, j}\right)}\left(\frac{\Omega_{i, j}}{m_{i, j}}\right)^{\ell_{i}}\left(\frac{\beta m_{0, j} \Omega_{i, j}}{m_{i, j} \Omega_{0, j}}+1\right)^{-\left(m_{i, j}+\ell_{i}\right)} .
$$

Finally, by substituting A.10 into A.8 and then into (2.8), 2.9 is obtained. 


\section{Appendix B}

\section{Asymptotic Outage Analysis}

This appendix provides details leading to 2.50 through two approach: 1) direct approach using classic probability theory; 2) stochastic geometry approach.

\subsection{Direct Approach}

Let $\mathcal{A}$ include the interferers on the entire plane $\mathbb{R}^{2}$. The outage probability can be found by taking the limit of 2.49 as $r_{\text {in }} \rightarrow 0$ and $r_{\text {out }} \rightarrow \infty$. Using (2.49), the limit may be expressed as

$$
\epsilon_{j}=1-\exp \left\{-\frac{\beta r_{0, j}^{\alpha}}{\mathrm{SNR}}+\pi \lambda p \lim _{x \rightarrow \infty} \Phi(x)\right\}
$$

where the right hand side follows from the continuity of the exponential function.

By performing the change of variables $y=\frac{\beta r_{0, j}^{\alpha}}{x^{\alpha}}$ and $v=1+\frac{2}{\alpha}$, the limit in B.1) may be written as

$$
\begin{aligned}
\lim _{x \rightarrow \infty} \Phi(x) & =\lim _{y \rightarrow 0} \Phi\left(\frac{\beta^{\frac{1}{\alpha}} r_{0, j}}{y^{\frac{1}{\alpha}}}\right) \\
& =\beta^{v-1} r_{0, j}^{2}(v-1) \lim _{y \rightarrow 0}\left\{y^{1-v} \phi(y)\right\}
\end{aligned}
$$

where

$$
\phi(y)=\frac{1}{v y}{ }_{2} F_{1}\left([1, v] ; v+1,-y^{-1}\right)-\frac{1}{v-1} .
$$

From 2.24) and the functional equation $\Gamma(1+x)=x \Gamma(x)$,

$$
\phi(y)=\frac{1}{y} \int_{0}^{1} t^{v-1}\left(1+\frac{t}{y}\right)^{-1} d t-\frac{1}{v-1} .
$$


By splitting the integral at $y$ and factoring the second integrand,

$$
\begin{aligned}
\phi(y) & =\frac{1}{y} \int_{0}^{y} t^{v-1}\left(1+\frac{t}{y}\right)^{-1} d t \\
& +\int_{y}^{1} t^{v-2}\left(1+\frac{y}{t}\right)^{-1} d t-\frac{1}{v-1} .
\end{aligned}
$$

By substituting the Taylor series and then integrating term by term,

$$
\phi(y)=\frac{1}{y} \sum_{i=0}^{\infty} \frac{(-1)^{i} y^{v}}{v+i}+\sum_{i=0}^{\infty} \frac{(-1)^{i}\left(y^{i}-y^{v-1}\right)}{v-1-i}-\frac{1}{v-1} .
$$

Simplifying the first series and dividing the second series into two series,

$$
\begin{aligned}
\phi(y)= & \sum_{i=0}^{\infty} \frac{(-1)^{i} y^{v-1}}{v+i}+\sum_{i=0}^{\infty} \frac{(-1)^{i} y^{i}}{v-1-i} \\
& -\sum_{i=0}^{\infty} \frac{(-1)^{i} y^{v-1}}{v-1-i}-\frac{1}{v-1} .
\end{aligned}
$$

Combining the first and third series, canceling the first term of the second series, and multiplying both sides by $y^{1-v}$,

$$
\phi(y) y^{1-v}=\sum_{i=0}^{\infty}\left\{\frac{(-1)^{i}}{v+i}-\frac{(-1)^{i}}{v-1-i}\right\}+\sum_{i=1}^{\infty} \frac{(-1)^{i} y^{i-v+1}}{v-1-i} .
$$

Taking the limit of $(\overline{B .8})$ as $y \rightarrow 0$

$$
\begin{aligned}
\lim _{y \rightarrow 0}\left\{\sum_{i=0}^{\infty}\left\{\frac{(-1)^{i}}{v+i}-\frac{(-1)^{i}}{v-1-i}\right\}+\sum_{i=1}^{\infty} \frac{(-1)^{i} y^{i-v+1}}{v-1-i}\right\} \\
=\sum_{i=0}^{\infty}\left\{\frac{(-1)^{i}}{v+i}-\frac{(-1)^{i}}{v-1-i}\right\}+\sum_{i=1}^{\infty} \frac{(-1)^{i} \lim _{y \rightarrow 0} y^{i-v+1}}{v-1-i}
\end{aligned}
$$

where the second line follows from the fact that the first series is independent of $y$ and finite. Since $\alpha>2$, and therefore $v<2$, the exponent of $y^{i-v+1}$ is always positive. Therefore, the limit in (B.9) evaluates to zero, and

$$
\lim _{y \rightarrow 0} \phi(y) y^{1-v}=\sum_{i=0}^{\infty}\left\{\frac{(-1)^{i}}{v+i}-\frac{(-1)^{i}}{v-1-i}\right\} .
$$

Substituting B.10 into B.2

$$
\lim _{x \rightarrow \infty} \Phi(x)=\beta^{v-1} r_{0}^{2}(v-1)\left\{\sum_{i=0}^{\infty} \frac{(-1)^{i}}{v+i}-\sum_{i=0}^{\infty} \frac{(-1)^{i}}{v-1-i}\right\} .
$$


Consider the first series in (B.11). By performing the change of variables $i=-j-1$,

$$
\sum_{i=0}^{\infty} \frac{(-1)^{i}}{v+i}=\sum_{j=-\infty}^{-1} \frac{(-1)^{-j-1}}{v-j-1} \Rightarrow-\sum_{i=-\infty}^{-1} \frac{(-1)^{i}}{v-1-i} .
$$

Substituting (B.12) into (B.11), combining the two series, and replacing $(v-1)$ with $2 / \alpha$,

$$
\lim _{x \rightarrow \infty} \Phi(x)=-\frac{2}{\alpha} \beta^{2 / \alpha} r_{0}^{2} \sum_{i=-\infty}^{\infty} \frac{(-1)^{i}}{\frac{2}{\alpha}-i} .
$$

The series in (B.13) can be written as

$$
\begin{aligned}
\sum_{i=-\infty}^{\infty} \frac{(-1)^{i}}{\frac{2}{\alpha}-i} & =\frac{\alpha}{2}+2\left(\frac{2}{\alpha}\right) \sum_{i=1}^{\infty} \frac{(-1)^{i}}{\left(\frac{2}{\alpha}\right)^{2}+i^{2}} \\
& =\pi \csc \left(\frac{2 \pi}{\alpha}\right) .
\end{aligned}
$$

where the last line follows from (4.22.5) of [156]. Substituting (B.14) into (B.13),

$$
\frac{1}{\frac{2}{\alpha}+i}+\frac{1}{\frac{2}{\alpha}-i}=\frac{2\left(\frac{2}{\alpha}\right)}{\left(\frac{2}{\alpha}\right)^{2}+i^{2}}
$$

and combining the two series,

$$
\lim _{x \rightarrow \infty} \Phi(x)=-\frac{2 \beta^{2 / \alpha} r_{0, j}^{2}}{\alpha}\left\{\frac{\alpha}{2}+2\left(\frac{2}{\alpha}\right) \sum_{i=1}^{\infty} \frac{(-1)^{i}}{\left(\frac{2}{\alpha}\right)^{2}+i^{2}}\right\} .
$$

From (4.22.5) of [156],

$$
\lim _{x \rightarrow \infty} \Phi(x)=-\frac{2 \pi}{\alpha} \beta^{2 / \alpha} r_{0, j}^{2} \csc \left(\frac{2 \pi}{\alpha}\right)
$$

Finally, substituting (B.17) into (B.1) gives 2.50 . 


\subsection{Stochastic Geometry Approach}

Let $\Phi(\lambda p)=\Omega_{i}$ denote the location of the interferers, which are distributed according to a $\mathrm{PPP}$ with intensity $\lambda p$. The outage probability is

$$
\begin{aligned}
\epsilon_{j} & =1-\mathbb{P}\left[\frac{g_{0, j} r_{0, j}^{-\alpha}}{\mathrm{SNR}^{-} 1+\sum_{i \in \Phi(\lambda p)} g_{i, j} r_{i, j}^{-\alpha}}>\beta\right] \\
& =1-\mathbb{P}\left[g_{0}>\frac{\beta r_{0, j}^{\alpha}}{\mathrm{SNR}}+\beta r_{0, j}^{\alpha} \sum_{i \in \Phi(\lambda p)} g_{i, j} r_{i, j}^{-\alpha}\right] \\
& \stackrel{(a)}{=} 1-\exp \left\{-\frac{\beta r_{0, j}^{\alpha}}{\mathrm{SNR}}\right\} \mathbb{E}_{\boldsymbol{g}, \Phi}\left[\exp \left\{s \sum_{i \in \Phi(\lambda p)} g_{i, j} r_{i, j}^{-\alpha}\right\}\right]_{s=\beta r_{0, j}^{\alpha}} \\
& \stackrel{(b)}{=} 1-\exp \left\{-\frac{\beta r_{0, j}^{\alpha}}{\mathrm{SNR}}\right\} \mathbb{E}_{\Phi}\left[\prod_{i \in \Phi(\lambda p)} \mathbb{E}_{g_{i, j}}\left[\exp \left\{s g_{i, j} r_{i}^{-\alpha}\right\}\right]_{s=\beta r_{0, j}^{\alpha}}\right.
\end{aligned}
$$

where $\mathbb{E}_{g_{i, j}}[\cdot]$ and $\mathbb{E}_{\Phi}[\cdot]$ are the operator expectation evaluate respectively over the fading and the spatial distribution of the interferers, $\stackrel{(a)}{=}$ follows due to the fact that $g_{0, j}$ is exponentially distributed, while $\stackrel{(b)}{=}$ follows due to the fact that $\left\{g_{i, j}\right\}$ and $\left\{r_{i, j}\right\}$ are independent.

Since $g_{i, j}$ is exponentially distributed, the inner expectation in (B.21) is

$$
\begin{aligned}
\mathbb{E}_{g_{i, j}}\left[\exp \left\{-s g_{i, j} r_{i, j}^{-\alpha}\right\}\right] & =\int_{0}^{\infty} \exp \left\{-g_{i, j}\left(s r_{i, j}^{-\alpha}+1\right)\right\} \mathrm{d} g_{i, j} \\
& =\frac{1}{1+s r_{i, j}^{-\alpha}} .
\end{aligned}
$$

Substituting (B.22) in (B.21), and using 157, proposition 2.12] for the PGFL of a PPP, the outer expectation in (B.21) is

$$
\mathbb{E}_{\Phi}\left[\prod_{i \in \Phi(\lambda p)} \frac{1}{1+s r_{i, j}^{-\alpha}}\right]=\exp \left\{-2 \pi \lambda p \int_{0}^{\infty} \frac{s r^{-\alpha}}{1+s r^{-\alpha}} r \mathrm{~d} r\right\}
$$

Performing the change of variables $u=s^{-\frac{1}{\alpha}} r$ and some algebraic manipulations, yields

$$
\begin{aligned}
\mathbb{E}_{\Phi}\left[\prod_{i \in \Phi(\lambda p)} \frac{1}{1+s r_{i, j}^{-\alpha}}\right] & =\exp \left\{-2 \pi \lambda p s^{2 / \alpha} \int_{0}^{\infty} \frac{u}{1+u^{\alpha}} \mathrm{d} u\right\} \\
& =\exp \left\{-2 \pi \lambda p s^{2 / \alpha} \frac{\pi}{\alpha} \csc \left(\frac{2 \pi}{\alpha}\right)\right\} .
\end{aligned}
$$

Finally, recalling that $s=\beta r_{0, j}^{\alpha}$, and substituting (B.24) in (B.21), (2.50) is obtained. 


\section{Appendix C}

\section{Correlation Coefficient in a BPP: Derivation}

This appendix provides details leading to 2.56), 2.57) and (2.58). The evaluation of the three expressions requires the $\mathrm{PDF}$ of $\left\{r_{i, j}\right\}$, which in this case is 48

$$
f_{r}(r)= \begin{cases}\frac{2 \pi r}{|\mathcal{A}|} & \text { for } 0 \leq r \leq r_{\text {out }}-r_{0} \\ \frac{2 r}{|\mathcal{A}|} \arccos \left(\frac{r^{2}+r_{0}^{2}-r_{\text {out }}^{2}}{2 r_{0} r}\right) & \text { for } r_{\text {out }}-r_{0} \leq r \leq r_{\text {out }}+r_{0}\end{cases}
$$

\subsection{Spatially Averaged First Moment}

The spatially averaged first moment of the outage probability is derived starting from (7.9). In particular, substituting (C.1) in (7.9), yields

$$
\mathbb{E}_{\boldsymbol{\Omega}}\left[\epsilon_{j}(\boldsymbol{\Omega}) \mid M\right]=1-\exp \left(\frac{\beta r_{0}^{\alpha}}{\mathrm{SNR}}\right)\left[1-p+\frac{2 \pi p}{|\mathcal{A}| \beta r_{0}^{\alpha}} \int_{0}^{r_{\text {out }}-r_{0}} \frac{r^{\alpha+1}}{1+r^{\alpha} \beta^{-1} r_{0}^{-\alpha}} \mathrm{d} r+\mathcal{Z}_{i}\left(r_{\text {out }}, r_{0}\right)\right]^{M}(\mathrm{C}
$$

where

$$
\mathcal{Z}_{i}\left(r_{\text {out }}, r_{0}\right)=\int_{r_{\text {out }}-r_{0}}^{r_{\text {out }}+r_{0}} \frac{r^{i \alpha+1}}{\left(\beta r_{0}^{\alpha}+r^{\alpha}\right)^{i}} \arccos \left(\frac{r^{2}+r_{0}^{2}-r_{\text {out }}^{2}}{2 r_{0} r}\right) \mathrm{d} r
$$

Since the first integral in (C.2) is 2.19, which leads to 2.21), and after few algebraic manipulations, 2.56 is obtained. 


\subsection{Spatially Averaged Second Moment}

The spatially averaged second moment of the outage probability is derived starting from (2.53) substituting in (2.10). Since $\Omega=r^{-\alpha}$ and the $M$ interferers are independently and uniformly distributed (i.u.d.) over $\mathcal{A}$, yields

$$
\begin{aligned}
\mathbb{E}_{\boldsymbol{\Omega}}\left[\epsilon_{j}^{2}(\boldsymbol{\Omega}) \mid M\right]= & 1-2 \exp \left(-\frac{\beta r_{0}^{\alpha}}{\mathrm{SNR}}\right)\left(1-p+p \int f_{r}(r) \frac{r^{\alpha}}{\beta r_{0}^{\alpha}+r^{\alpha}} \mathrm{d} r\right)^{M} \\
& +\exp \left(-2 \frac{\beta r_{0}^{\alpha}}{\mathrm{SNR}}\right)\left[\int f_{r}(r)\left(1-p+\frac{p r^{\alpha}}{\beta r_{0}^{\alpha}+r^{\alpha}}\right)^{2} \mathrm{~d} r\right]^{M} .
\end{aligned}
$$

By substituting (C.1) in (C.4), from (2.19) and (C.3) the first integral in (C.4) is

$$
\int f_{r}(r) \frac{r^{\alpha}}{\beta r_{0}^{\alpha}+r^{\alpha}} \mathrm{d} r=\frac{2}{|\mathcal{A}|}\left[\frac{\pi}{\beta r_{0}^{\alpha}} \Psi_{1}\left(r_{\text {out }}-r_{0}\right)+\mathcal{Z}_{1}\left(r_{\text {out }}, r_{0}\right)\right]
$$

while after few algebraic manipulations the second integral is

$$
\begin{aligned}
\mathcal{S}\left(r_{\text {out }}, r_{0}\right)= & \int f_{r}(r)\left(1-p+\frac{p r^{\alpha}}{\beta r_{0}^{\alpha}+r^{\alpha}}\right)^{2} \mathrm{~d} r \\
= & (1-p)^{2}+\frac{4}{|\mathcal{A}|}(1-p) p\left[\frac{\pi}{\beta r_{0}^{\alpha}} \Psi_{1}\left(r_{\text {out }}-r_{0}\right)+\mathcal{Z}_{1}\left(r_{\text {out }}, r_{0}\right)\right] \\
& +\frac{2 p^{2}}{|\mathcal{A}|}\left[\pi \mathcal{G}\left(r_{\text {out }}-r_{0}\right)+\mathcal{Z}_{2}\left(r_{\text {out }}, r_{0}\right)\right]
\end{aligned}
$$

where $\mathcal{Z}_{i}\left(r_{\text {out }}, r_{0}\right)$ is given by $($ C.3 $)$ and

$$
\mathcal{G}(x)=\int_{0}^{x} \frac{r^{2 \alpha+1}}{\left(\beta r_{0}^{\alpha}+r^{\alpha}\right)^{2}} \mathrm{~d} r .
$$

By performing the change of variable $r=y \nu^{\frac{1}{\alpha}}$,

$$
\mathcal{G}(x)=\frac{x^{2 \alpha+2}}{(2+2 \alpha) \beta^{2} r_{0}^{2 \alpha}{ }_{2}} F_{1}\left(\left[2, \frac{2+2 \alpha}{\alpha}\right] ; \frac{2+3 \alpha}{\alpha} ;-\frac{x^{\alpha}}{\beta r_{0}^{\alpha}}\right) .
$$

By substituting (C.5), (C.6) and (C.8), in (C.4), and by performing few algebraic manipulations 2.57) is obtained.

\subsection{Spatially Averaged Joint First Moment}

The spatially averaged joint first moment of the outage probability is derived starting from 2.54). If polar coordinates are used to evaluate the distances between each interferer 
and the $j^{\text {th }}$ receiver, then

$$
\boldsymbol{r}(\rho, \phi, \theta)=\left\|r_{0} \exp (j \theta)+r_{\text {out }} \sqrt{\rho} \exp (j \phi)\right\|
$$

where $\rho \sim \mathcal{U}(0,1)$ and $\phi \sim \mathcal{U}(0,2 \pi)$. Since the $M$ interferers are independently and uniformly distributed (i.u.d.) over $\mathcal{A}$,

$$
\begin{aligned}
\mathbb{E}_{\boldsymbol{\Omega}}\left[\epsilon_{1}(\boldsymbol{\Omega}) \epsilon_{2}(\boldsymbol{\Omega}) \mid M\right]=1 & -\exp \left(-\frac{\beta r_{0}^{\alpha}}{\mathrm{SNR}}\right)\left(1-p+p \iint \frac{r^{\alpha}(\rho, \phi, 0)}{\beta r_{0}^{\alpha}+r^{\alpha}(\rho, \phi, 0)} f_{\rho}(\rho) f_{\phi}(\phi) \mathrm{d} \rho \mathrm{d} \phi\right)^{M} \\
& -\exp \left(-\frac{\beta r_{0}^{\alpha}}{\mathrm{SNR}}\right)\left(1-p+p \iint \frac{r^{\alpha}(\rho, \phi, \theta)}{\beta r_{0}^{\alpha}+r^{\alpha}(\rho, \phi, \theta)} f_{\rho}(\rho) f_{\phi}(\phi) \mathrm{d} \rho \mathrm{d} \phi\right)^{M} \\
& +\exp \left(-2 \frac{\beta r_{0}^{\alpha}}{\mathrm{SNR}}\right)\left[\iint\left(1-p+\frac{p r^{\alpha}(\rho, \phi, 0)}{\beta r_{0}^{\alpha}+r^{\alpha}(\rho, \phi, 0)}\right)\right. \\
& \left.\times\left(1-p+\frac{p r^{\alpha}(\rho, \phi, \theta)}{\beta r_{0}^{\alpha}+r^{\alpha}(\rho, \phi, \theta)}\right) f_{\rho}(\rho) f_{\phi}(\phi) \mathrm{d} \rho \mathrm{d} \phi\right]^{M} . \quad(\mathrm{C} .10)
\end{aligned}
$$

The first and second double integral in C.10 are equivalent, since the first moment of the outage probability is the same at the two receivers, which are equally distant from the reference transmitter, and they coincide with (C.5). Performing the cross products and several other algebraic manipulations in the last term of (C.10) and using the functions given by 7.10 and (C.3), yields

$$
\begin{aligned}
\mathcal{W}\left(r_{\text {out }}, r_{0}, \theta\right) & =\iint\left(1-p+\frac{p r^{\alpha}(\rho, \phi, 0)}{\beta r_{0}^{\alpha}+r^{\alpha}(\rho, \phi, 0)}\right)\left(1-p+\frac{p r^{\alpha}(\rho, \phi, \theta)}{\beta r_{0}^{\alpha}+r^{\alpha}(\rho, \phi, \theta)}\right) \mathrm{d} \rho \mathrm{d} \phi \\
& =(1-p)^{2}+\frac{4}{|\mathcal{A}|}(1-p) p\left[\pi \Psi_{1}\left(r_{\text {out }}-r_{0}\right)+\mathcal{Z}_{1}\left(r_{\text {out }}, r_{0}\right)\right]+p^{2} \mathcal{X}\left(r_{\text {out }}, r_{0}, \theta\right)
\end{aligned}
$$

where

$$
\mathcal{X}\left(r_{\text {out }}, r_{0}, \theta\right)=\frac{1}{2 \pi} \int_{0}^{1} \int_{0}^{2 \pi} \frac{r^{\alpha}(\rho, \phi, 0)}{\beta r_{0}^{\alpha}+r^{\alpha}(\rho, \phi, 0)} \frac{r^{\alpha}(\rho, \phi, \theta)}{\beta r_{0}^{\alpha}+r^{\alpha}(\rho, \phi, \theta)} \mathrm{d} \rho \mathrm{d} \phi .
$$

Finally, By substituting (C.11), and (C.12), in C.10), and by performing few algebraic manipulations (2.58) is obtained. 


\section{Appendix D}

\section{Conditional Outage Probability for Diversity Combining: Derivation}

This appendix provides details leading to 4.9 . In the absence of interference, $\epsilon_{j}(\boldsymbol{\Omega})=$ $F_{\mathrm{S}}\left(\mathrm{SNR}^{-1} \mid \Omega\right)$. Restricting the Nakagami parameters $m_{k, j}, \forall k \in \mathcal{G}_{j}$, to be integer-valued, but not necessarily identical, the CDF of $\mathrm{S}$ is found in [158] to be

$$
\begin{aligned}
F_{\mathrm{S}}\left(s \mid \mathbf{\Omega}_{j}\right)= & \sum_{k \in \mathcal{G}_{j}} \sum_{n=1}^{m_{k, j}}\left[1-\exp \left(-\frac{\beta m_{k, j} s}{\Omega_{k, j}}\right) \sum_{\mu=0}^{n-1} \frac{\left(\beta m_{k, j} s\right)^{\mu}}{\Omega_{k, j}^{\mu} \mu !}\right] \\
& \Xi_{\mathcal{N}_{j}}\left(k, n,\left\{m_{q, j}\right\}_{\forall q \in \mathcal{G}_{j}},\left\{\frac{\Omega_{q, j}}{\beta m_{q, j}}\right\}_{\forall q \in \mathcal{G}_{j}}\right) .
\end{aligned}
$$

The function $\Xi_{L}\left(k, n,\left\{r_{q}\right\}_{q=1}^{L},\left\{\eta_{q}\right\}_{q=1}^{L}\right)$ is

$$
\begin{aligned}
& \Xi_{L}\left(k, n,\left\{r_{q}\right\}_{q=1}^{L},\left\{\eta_{q}\right\}_{q=1}^{L}\right)=\sum_{l_{1}=n}^{r_{k}} \sum_{l_{2}=n}^{l_{1}} \ldots \sum_{l_{L-2}=n}^{l_{L-3}}\left[\frac{(-1)^{R_{L}-r_{k}} \eta_{k}^{n}}{\prod_{h=1}^{L} \eta_{h}^{r_{h}}} \frac{\left(r_{k}+r_{1+u(1-k)}-l_{1}-1\right) !}{\left(r_{1+u(1-k)}-1\right) !\left(r_{k}-l_{1}\right) !}\right. \\
& \left(\frac{1}{\eta_{j}}-\frac{1}{\eta_{1+u(1-k)}}\right)^{l_{1}-r_{k}-r_{1+u(1-k)}} \frac{\left(l_{L-2}+r_{L-1+u(L-1-k)}-n-1\right) !}{\left(r_{L-1+u(L-1-k)}-1\right) !\left(l_{L-2}-n\right) !} \\
& \left(\frac{1}{\eta_{k}}-\frac{1}{\eta_{L-1+u(L-1-k)}}\right)^{n-l_{L-2}-r_{L-1+u(L-1-k)}} \prod_{s=1}^{L-3} \frac{\left(l_{s}+r_{s+1+u(s+1-k)}-l_{s+1}-1\right) !}{\left(r_{s+1+u(s+1-k)}-1\right) !\left(l_{s}-l_{s+1}\right) !} \\
& \left(\frac{1}{\eta_{k}}-\frac{1}{\eta_{s+1+u(s+1-k)}}\right)^{l_{s+1}-l_{s}-r_{s+1+u(s+1-k)}}
\end{aligned}
$$

where $u(x)$ is the step function and

$$
R_{L}=\sum_{k=1}^{L} r_{k}
$$


The CDF of $Z_{j}$ can be computed as follows:

$$
F_{\mathbf{Z}_{j}}(z \mid \Omega)=P\left[\mathbf{Z}_{j}<z\right]=P\left[\mathrm{~S}_{j}<z+\sum_{i \notin \mathcal{G}_{j}} \mathbf{Y}_{i, j}\right]=\iint_{\mathbb{R}^{M-\mathcal{N}_{j}}} \ldots \int_{\mathbf{Y}}(\mathbf{y}) F_{\mathbf{S}}\left(z+\sum y_{i}\right) \mathrm{d} \mathbf{y}
$$

where the outer integral is over $\left(M-\mathcal{N}_{j}\right)$-dimensional real space and $f_{\mathbf{Y}}(\mathbf{y})$ is the joint $\mathrm{PDF}$ of the vector $\mathbf{Y}$, where each element is gamma-distributed with Nakagami parameter $m_{i, j}$. Substituting (D.1) into (D.4) and performing a few manipulations yields

$$
\begin{aligned}
F_{\mathbf{Z}_{j}}(z \mid \Omega)= & \sum_{k \in \mathcal{G}_{j}} \sum_{n=1}^{m_{k, j}} \Xi_{N_{j}}\left(k, n,\left\{m_{q, j}\right\}_{\forall q \in \mathcal{G}_{j}},\left\{\frac{\Omega_{q, j}}{\beta m_{q, j}}\right\}_{\forall q \in \mathcal{G}_{j}}\right)\left\{1-\exp \left(-\frac{\beta m_{k, j} z}{\Omega_{k, j}}\right)\right. \\
& \left.\sum_{\mu=0}^{n-1} \frac{\left(\beta m_{k, j} z\right)^{\mu}}{\Omega_{k, j}^{\mu} \mu !} \int_{\mathbb{R}^{M-\mathcal{N}_{j}}} \ldots \int_{\mathbf{Y}}(\mathbf{y}) \exp \left(-\frac{\beta m_{k, j}}{\Omega_{k, j}} \sum y_{i}\right)\left(1+z^{-1} \sum_{i \notin \mathcal{G}_{j}} y_{i}\right)^{\mu} \mathrm{d} \mathbf{y}\right\} .
\end{aligned}
$$

Since $\mu$ is an integer, substituting (A.6) and A.7) into (D.5), performing a few manipulations, and assuming that the interfering channels fade independently, it follows that

$$
\begin{aligned}
F_{\mathbf{Z}_{j}}(z \mid \Omega)= & \sum_{k \in \mathcal{G}_{j}} \sum_{n=1}^{m_{k, j}} \Xi_{N_{j}}\left(k, n,\left\{m_{q, j}\right\}_{\forall q \in \mathcal{G}_{j}},\left\{\frac{\Omega_{q, j}}{\beta m_{q, j}}\right\}_{\forall q \in \mathcal{G}_{j}}\right)\left\{1-\exp \left(-\frac{\beta m_{k, j} z}{\Omega_{k, j}}\right)\right. \\
& \left.\times \sum_{\mu=0}^{n-1} \frac{\left(\beta m_{k, j} z\right)^{\mu}}{\Omega_{k, j}^{\mu} \mu !} \sum_{t=0}^{\mu} \frac{z^{-t}}{(\mu-t) !} \sum_{\substack{\ell_{i} \geq 0 \\
\sum_{i=0}^{M-\mathcal{N}_{j}} \ell_{i}=t}}^{\prod_{i \notin \mathcal{G}_{j}}}\right\}
\end{aligned}
$$

where

$$
G_{\ell_{i}}\left(\Omega_{i, j}\right)=\int_{\mathbb{R}} \exp \left(-\frac{\beta m_{k, j} y}{\Omega_{k, j}}\right) \frac{y^{\ell_{i}}}{\ell_{i} !} f_{\mathrm{Y}_{i, j}}(y) \mathrm{d} y .
$$

Substituting (A.2), in (D.7), it yields

$$
G_{\ell_{i}}\left(\Omega_{i, j}\right)=\left(1-p_{i}\right) \delta_{\ell_{i}}+\frac{p_{i} \Gamma\left(\ell_{i}+m_{i, j}\right)}{\ell_{i} ! \Gamma\left(m_{i, j}\right)}\left(\frac{\Omega_{i, j}}{m_{i, j}}\right)^{\ell_{i}}\left(\frac{\beta m_{k, j}}{\Omega_{k, j}} \frac{\Omega_{i, j}}{m_{i, j}}+1\right)^{-\left(m_{i, j}+\ell_{i}\right)} .
$$

Finally, by substituting (D.8) into (D.6), 4.9) is obtained. 


\section{Appendix E}

\section{Expectation and Variance of the Decoding Complexity: Derivation}

This appendix provides details leading to the expectation and the variance of the decoding complexity, given respectively by (5.15) and (5.16).

\subsection{Expectation of the Decoding Complexity}

By knowing the $\mathrm{PDF}$ of the $\gamma$ and by using the complexity model in Section 5.2.2, the expected decoding complexity conditioned on the average SNR can be evaluated as follows

$$
\begin{aligned}
\mathbb{E}_{\gamma_{i}, \Delta \gamma \mid \bar{\gamma}}\left\{\mathcal{C}_{i}\right\} & =\int_{0}^{\infty} \mathcal{C}_{i}(\gamma, \Delta \gamma) f_{\gamma_{i}}(\gamma) d \gamma \\
& =\sum_{k=1}^{N_{R}} \int_{\gamma_{k}^{R}}^{\gamma_{k+1}^{R}} \mathcal{C}_{i}(\gamma, \Delta \gamma) f_{\gamma_{i}}(\gamma) d \gamma
\end{aligned}
$$

By substituting (5.14) and (5.3) into E.1), the integral in (E.1) becomes

$$
\int_{\gamma_{k}^{R}}^{\gamma_{k+1}^{R}} \mathcal{C}_{i}(\gamma, \Delta \gamma) f_{\gamma_{i}}(\gamma) d \gamma=\frac{\mathcal{R}\left(\gamma_{k}^{R}, \Delta \gamma\right)}{\log _{2}(\zeta-1)}\left[\mathcal{I}_{1}\left(\gamma_{k+1}^{R}, \gamma_{k}^{R}\right)+\mathcal{I}_{2}\left(\gamma_{k+1}^{R}, \gamma_{k}^{R}\right)\right]
$$


where

$$
\begin{aligned}
\mathcal{I}_{1}\left(x_{1}, x_{2}\right) & =\log _{2}\left(\frac{\zeta-2}{K \zeta}\right) \int_{x_{2}}^{x_{1}} \frac{1}{\bar{\gamma}} \exp \left(-\frac{\gamma}{\bar{\gamma}}\right) d \gamma \\
& =-\left.\log _{2}\left(\frac{\zeta-2}{K \zeta}\right) \exp \left(-\frac{\gamma}{\bar{\gamma}}\right)\right|_{x_{2}} ^{x_{1}} \\
\mathcal{I}_{2}\left(x_{1}, x_{2}\right) & =\frac{-2}{\bar{\gamma}} \int_{x_{2}}^{x_{1}} \log _{2}\left[l_{k}\left(\gamma, R_{k}\right)\right] \exp \left(-\frac{\gamma}{\bar{\gamma}}\right) d \gamma
\end{aligned}
$$

with

$$
l_{k}\left(\gamma, R_{k}\right)=\log _{2}(1+\gamma)-\mathcal{R}\left(\gamma_{k}^{R}, \Delta \gamma\right)
$$

Eq. (E.4) cannot be solved in closed form. However, a piece-wise linearization of (E.5) can be done, which leads to

$$
l_{k}\left(\gamma, R_{k}\right) \approx c_{k}\left(\gamma, R_{k}\right)=a_{k} \gamma+b_{k}
$$

where

$$
\begin{aligned}
a_{k} & =\frac{l_{k}\left(\gamma_{k+1}^{R}, R_{k}\right)-l_{k}\left(\gamma_{k}^{R}, R_{k}\right)}{\gamma_{k+1}^{R}-\gamma_{k}^{R}} \\
b_{k} & =l_{k}\left(\gamma_{k}^{R}, R_{k}\right)-\frac{\gamma_{k}^{R}}{\gamma_{k+1}^{r}-\gamma_{k}^{R}}\left[l_{k}\left(\gamma_{k+1}^{R}, R_{k}\right)-l_{k}\left(\gamma_{k}^{R}, R_{k}\right)\right] .
\end{aligned}
$$

Substituting (E.6) into (E.4) and by integrating by parts yields

$$
\left.\mathcal{I}_{2}\left(x_{1}, x_{2}\right) \approx 2 \log _{2}\left[c_{k}\left(\gamma, R_{k}\right)\right] \exp \left(-\frac{\gamma}{\bar{\gamma}}\right)\right|_{x_{2}} ^{x_{1}}-\mathcal{I}_{3}\left(x_{1}, x_{2}\right)
$$

where

$$
\begin{aligned}
\mathcal{I}_{3}\left(x_{1}, x_{2}\right) & =2 \int_{x_{2}}^{x_{1}}\left\{\frac{\partial}{\partial \gamma} \log _{2}\left[l_{k}\left(\gamma, R_{k}\right)\right]\right\} \exp \left(-\frac{\gamma}{\bar{\gamma}}\right) d \gamma \\
& =\frac{2}{\log (2)} \int_{x_{2}}^{x_{1}} \frac{a_{k}}{a_{k} \gamma+b_{k}} \exp \left(-\frac{\gamma}{\bar{\gamma}}\right) d \gamma
\end{aligned}
$$


After few algebraic manipulations and using the change of variable $\gamma=\frac{a_{k} t+b_{k}}{a_{k} \bar{\gamma}}$, E.10 becomes

$$
\mathcal{I}_{3}\left(x_{1}, x_{2}\right)=\frac{2}{\log (2)} \exp \left(\frac{b_{k}}{a_{k} \bar{\gamma}}\right)\left[\int_{-\tau\left(x_{1}\right)}^{\infty} \frac{1}{t} \exp (-t) d t-\int_{-\tau\left(x_{2}\right)}^{\infty} \frac{1}{t} \exp (-t) d t\right]
$$

where $\tau(x)=\frac{a_{k} x+b_{k}}{a_{k} \bar{\gamma}}$.

The integrals in (E.11) are exponential integrals, defined as (i.e, 112])

$$
\mathrm{E}_{1}(x)=\int_{x}^{\infty} \frac{e^{-t}}{t} d t
$$

By substituting (E.12) into (E.11), and (E.11) into (E.9) yields

$$
\left.\mathcal{I}_{2}\left(x_{1}, x_{2}\right) \approx 2 \log _{2}\left[c_{k}\left(\gamma, R_{k}\right)\right] \exp \left(-\frac{\gamma}{\bar{\gamma}}\right)\right|_{x_{2}} ^{x_{1}}+\left.\frac{2}{\log (2)} \exp \left(\frac{b_{k}}{a_{k} \bar{\gamma}}\right) \mathrm{E}_{1}[\tau(x)]\right|_{x_{2}} ^{x_{1}} .
$$

Finally, an approximated closed form expression for the expected decoding complexity conditioned over the average $\underline{\mathrm{SNR}}$ is obtained by substituting $(\mathrm{E.2}),(\mathrm{E} .3)$, and $(\mathrm{E} .13)$ into E.1.

\subsection{Variance of the Decoding Complexity}

The variance of the decoding complexity conditioned over the average SNR is

$$
\begin{aligned}
\operatorname{Var}_{\gamma_{i}, \Delta \gamma \mid \bar{\gamma}}\left\{\mathcal{C}_{i}\right\} & =\mathbb{E}_{\gamma_{i}, \Delta \gamma \mid \bar{\gamma}}\left\{\left[\mathcal{C}_{i}-\mathbb{E}_{\gamma_{i}, \Delta \gamma \mid \bar{\gamma}}\left\{\mathcal{C}_{i}\right\}\right]^{2}\right\} \\
& =\mathbb{E}_{\gamma_{i}, \Delta \gamma \mid \bar{\gamma}}\left\{\mathcal{C}_{i}^{2}\right\}-\mathbb{E}_{\gamma_{i}, \Delta \gamma \mid \bar{\gamma}}\left\{\mathcal{C}_{i}\right\}^{2}
\end{aligned}
$$

where $\mathbb{E}_{\gamma_{i}, \Delta \gamma \mid \bar{\gamma}}\left\{\mathcal{C}_{i}\right\}$ is given by $(5.15)$ and

$$
\begin{aligned}
\mathbb{E}_{\gamma_{i}, \Delta \gamma \mid \bar{\gamma}}\left\{\mathcal{C}_{i}^{2}\right\} & =\int_{0}^{\infty} \mathcal{C}_{i}^{2}(\gamma, \Delta \gamma) f_{\gamma_{i}}(\gamma) d \gamma \\
& =\sum_{k=1}^{N_{R}} \int_{\gamma_{k}^{R}}^{\gamma_{k+1}^{R}} \mathcal{C}_{i}^{2}(\gamma, \Delta \gamma) f_{\gamma_{i}}(\gamma) d \gamma
\end{aligned}
$$

By substituting (5.14) and (5.3) into (E.15) yields

$$
\mathbb{E}_{\gamma_{i}, \Delta \gamma \mid \bar{\gamma}}\left\{\mathcal{C}_{i}^{2}\right\}=\sum_{k=1}^{N_{R}}\left[\frac{2 \mathcal{R}\left(\gamma_{k}^{R}, \Delta \gamma\right)}{\log _{2}(\zeta-1)}\right]^{2} I_{4}\left(\gamma_{k+1}^{R}, \gamma_{k}^{R}\right)
$$


where

$$
\mathcal{I}_{4}\left(x_{1}, x_{2}\right)=\frac{1}{\bar{\gamma}} \int_{x_{2}}^{x_{1}}\left\{\log _{2}\left[\frac{\log _{2}(1+\gamma)-\mathcal{R}(\gamma, \Delta \gamma)}{\sqrt{\frac{\zeta-2}{K \zeta}}}\right]\right\}^{2} \exp \left(-\frac{\gamma}{\bar{\gamma}}\right) d \gamma
$$

The integral in (E.17) cannot be solved in closed form. However, a piece-wise linearization of (E.5) can be done, and E.17) becomes

$$
\mathcal{I}_{4}\left(x_{1}, x_{2}\right) \approx \frac{1}{\bar{\gamma}} \int_{x_{2}}^{x_{1}}\left\{\log _{2}\left(d_{k} \gamma+e_{k}\right)\right\}^{2} \exp \left(-\frac{\gamma}{\bar{\gamma}}\right) d \gamma
$$

where

$$
\begin{aligned}
& d_{k}=\frac{a_{k}}{\sqrt{\frac{\zeta-2}{K \zeta}}} \\
& e_{k}=\frac{b_{k}}{\sqrt{\frac{\zeta-2}{K \zeta}}} .
\end{aligned}
$$

By integrating (E.18) by parts yields

$$
\mathcal{I}_{4}\left(x_{1}, x_{2}\right) \approx-\left.\left[\log _{2}\left(d_{k} \gamma+e_{k}\right)\right]^{2} \exp \left(-\frac{\gamma}{\bar{\gamma}}\right)\right|_{x_{2}} ^{x_{1}}+\mathcal{I}_{5}\left(x_{1}, x_{2}\right)
$$

where

$$
\begin{aligned}
\mathcal{I}_{5}\left(x_{1}, x_{2}\right) & =\int_{x_{2}}^{x_{1}} \exp \left(-\frac{\gamma}{\bar{\gamma}}\right) \frac{\partial}{\partial \gamma}\left[\log _{2}\left(d_{k} \gamma+e_{k}\right)\right]^{2} d \gamma \\
& =\frac{2}{[\log (2)]^{2}} \int_{x_{2}}^{x_{1}} \exp \left(-\frac{\gamma}{\bar{\gamma}}\right) \frac{d_{k}}{d_{k} \gamma+e_{k}} \log \left(d_{k} \gamma+e_{k}\right) d \gamma
\end{aligned}
$$

After making the substitution $t=d_{k} \gamma+e_{k}$ into (E.22) yields

$$
\mathcal{I}_{5}\left(x_{1}, x_{2}\right)=\frac{2}{[\log (2)]^{2}} \exp \left(\frac{e_{k}}{d_{k} \bar{\gamma}}\right) \int_{d_{k} x_{2}+e_{k}}^{d_{k} x_{1}+e_{k}} \exp \left(-\frac{t}{d_{k} \bar{\gamma}}\right) \frac{\log (t)}{t} d t .
$$

By integrating again by parts, E.23 becomes

$$
\mathcal{I}_{5}\left(x_{1}, x_{2}\right)=\frac{2 \exp \left(\frac{e_{k}}{d_{k} \bar{\gamma}}\right)}{[\log (2)]^{2}}\left[-\left.\mathrm{E}_{1}\left(\frac{t}{d_{k} \bar{\gamma}}\right) \log (t)\right|_{d_{k} x_{2}+e_{k}} ^{d_{k} x_{1}+e_{k}}+\mathcal{I}_{6}\left(d_{k} x_{1}+e_{k}, d_{k} x_{2}+e_{k}\right)\right]
$$


where $\mathrm{E}_{1}(\cdot)$ is an exponential integral given as $(\mathrm{E} .12)$ and

$$
\mathcal{I}_{6}\left(x_{1}, x_{2}\right)=\int_{x_{2}}^{x_{1}} \frac{1}{t} \mathrm{E}_{1}\left(\frac{t}{d_{k} \bar{\gamma}}\right) d t .
$$

The integral (E.25) can be solved using the equality [159, Eq. (18), Sec. 4.1],

$$
\int_{b}^{\infty} \frac{\mathrm{E}_{1}(a x)}{x} d x=\frac{1}{2}\left\{\left[\gamma_{e}+\log (a b)\right]^{2}+\zeta(2)\right\}+\sum_{n=1}^{\infty} \frac{(-a b)^{n}}{n ! n^{2}}
$$

where $\gamma_{e}$ is the Euler-Mascheroni constant and $\zeta(\cdot)$ is the Riemann zeta function defined as (i.e, 112 )

$$
\zeta(x)=\sum_{n=1}^{\infty} n^{-x}
$$

Furthermore, let use the identify

$$
\sum_{n=1}^{\infty} \frac{(-a x)^{n}}{n ! n^{2}}=-x_{3} F_{3}\left[\begin{array}{lll}
1 & 1 & 1 \\
2 & 2 & 2
\end{array} ; x\right]
$$

where, by using the Pochhammer symbol $(a)_{0}=1$ and $(a)_{n}=a(a+1)(a+2) \ldots(a+n-1), n \geq$ $1,{ }_{p} F_{q}\left(a_{1}, \ldots, a_{p} ; b_{1}, \ldots, b_{q} ; z\right)$ is the generalized hypergeometric function defined as (i.e, [112])

$$
{ }_{p} F_{q}\left[\begin{array}{lll}
a_{1} & \ldots & a_{p} \\
b_{1} & \ldots & b_{q}
\end{array} ; x\right]=\sum_{n=0}^{\infty} \frac{\left(a_{1}\right)_{n} \ldots\left(a_{p}\right)_{n}}{\left(b_{1}\right)_{n} \ldots\left(b_{q}\right)_{n}} \frac{x^{n}}{n !} .
$$

By applying (E.26) and (E.28) into (E.25) yields

$$
\mathcal{I}_{6}\left(x_{1}, x_{2}\right)=-\left.\frac{1}{2}\left\{\left[\gamma_{e}+\log \left(\frac{t}{d_{k} \bar{\gamma}}\right)\right]^{2}+\zeta(2)\right\}\right|_{x_{2}} ^{x_{1}}+\left.\frac{t}{d_{k} \bar{\gamma}}{ }^{3} F_{3}\left[\begin{array}{lll}
1 & 1 & 1 \\
2 & 2 & 2
\end{array} ;-\frac{t}{d_{k} \bar{\gamma}}\right]\right|_{x_{2}} ^{x_{1}} .
$$

While the generalized hypergeometric function is itself an infinite series as well as the Riemann zeta function, they are widely known and they are implemented as a single function call in most mathematical programming languages, including Matlab.

Finally, by substituting (E.24) and (E.30) into (E.21), by defining the function $h(x)=$ $d_{k} x+e_{k}$ and after few manipulations yields

$$
\begin{aligned}
\mathcal{I}_{4}\left(x_{1}, x_{2}\right) \approx & -\left.\left[\log _{2}(t)\right]^{2} \exp \left(-\frac{t}{\bar{\gamma}}\right)\right|_{h\left(x_{2}\right)} ^{h\left(x_{1}\right)}+\frac{2 \exp \left(\frac{e_{k}}{d_{k} \bar{\gamma}}\right)}{[\log (2)]^{2}}\left[-\left.\mathrm{E}_{1}\left(\frac{t}{d_{k} \bar{\gamma}}\right) \log (t)\right|_{h\left(x_{2}\right)} ^{h\left(x_{1}\right)}\right. \\
& \left.-\left.\frac{1}{2}\left\{\left[\gamma_{e}+\log \left(\frac{t}{d_{k} \bar{\gamma}}\right)\right]^{2}+\zeta(2)\right\}\right|_{h\left(x_{2}\right)} ^{h\left(x_{1}\right)}+\left.\frac{t}{d_{k} \bar{\gamma}} F_{3}\left[\begin{array}{lll}
1 & 1 & 1 \\
2 & 2 & 2
\end{array} ;-\frac{t}{d_{k} \bar{\gamma}}\right]\right|_{h\left(x_{2}\right)} ^{h\left(x_{1}\right)}\right] .
\end{aligned}
$$




\section{Appendix F}

\section{Max-Rate Optimization: Derivation}

This appendix provides details leading to the solution of the optimization problem given by (6.2). Since both (6.5) and the constrained functions in 6.2 have continuous first partial derivatives, this problem can be solved through the method of Lagrange multipliers. Given the Lagrange multipliers $\eta$ and $\Theta=\left\{\Theta_{1}, \ldots, \Theta_{N_{c}}\right\}$, the Lagrangian can be written as follows

$$
L(\boldsymbol{R}, \eta, \boldsymbol{\Theta})=-\sum_{R_{k} \in \boldsymbol{R}} R_{k}+\eta\left[\sum_{R_{k} \in \boldsymbol{R}} \mathcal{C}_{k}\left(\gamma_{k}, \Delta \gamma\right)-N_{\text {cloud }} \mathcal{C}_{\max }\right]-\operatorname{tr}\left[\boldsymbol{\Theta} \operatorname{diag}\left(R_{k}\right)\right]
$$

The partial derivative of the Lagrangian over $R_{k}$ is

$$
\begin{aligned}
\frac{\partial L(\boldsymbol{R}, \eta, \boldsymbol{\Theta})}{\partial R_{k}} & =-1+\eta \frac{\partial \mathcal{C}_{k}\left(\gamma_{k}, \Delta \gamma\right)}{\partial R_{k}}-\boldsymbol{\Theta} \\
& =-1+\eta\left(2 v_{k} R_{k}+o_{k}\right)-\boldsymbol{\Theta}
\end{aligned}
$$

Using the Karush-Kuhn-Tucker conditions, it follows that

$$
\begin{gathered}
\forall k: \frac{\partial L(\boldsymbol{R}, \eta, \boldsymbol{\Theta})}{\partial R_{k}}=0 \Longrightarrow 1+\Theta_{k}=\eta\left(2 \alpha R_{k}+o_{k}\right) \\
\sum_{R_{k} \in \boldsymbol{R}} \mathcal{C}_{k}\left(\gamma_{k}, \Delta \gamma\right) \leq N_{\text {cloud }} \mathcal{C}_{\max } \Longrightarrow \eta \geq 0 \\
\forall k: R_{k} \geq 0 \Longrightarrow \forall \Theta_{k} \geq 0: \Theta_{k} R_{k}=0
\end{gathered}
$$

First, assume that $R_{k} \neq 0 \Longrightarrow \Theta_{k}=0$, which follows from (F.4). Using (F.2) and setting $\Theta_{k}=0$, yields

$$
1=\eta\left(2 v_{k} R_{k}+o_{k}\right)
$$


From (F.5) using (F.3), it follows that (with $\eta \geq 0$ )

$$
R_{k}=\frac{1}{2 v_{k}}\left(\frac{1}{\eta}-o_{k}\right)^{+}
$$

Now, let consider the case when $\Theta_{k} \neq 0 \Longrightarrow R_{k}=0$, which again follows from (F.4). Using $(\overline{\mathrm{F} .2})$ and setting $R_{k}=0$, yields

$$
1+\Theta_{k}=\eta o_{k}
$$

From (F.7) and since in this case $\Theta_{k}>0$, it follows that

$$
\eta>\frac{1}{o_{k}}
$$

By combining (F.6) and (F.8), Theorem 6.2.1 is obtained. 


\section{References}

[1] Qualcomm Technologies, "The 1000x Data Challenge," 2014. Available online at https://www.qualcomm.com/invention/technologies/1000x.

[2] J. G. Andrews, S. Buzzi, C. Wan, S. V. Hanly, A. Lozano, A. C. K. Soong, and J. C. Zhang, "What will 5G be?," IEEE J. Select. Areas Commun., vol. 32, pp. 1065-1082, June 2014.

[3] P. Cardieri, "Modeling interference in wireless ad hoc networks," IEEE Comm. Survey \& Tuts., vol. 12, pp. 551-572, Fourth Quarter 2010.

[4] M. Haenggi, "Outage and throughput bounds for stochastic wireless networks," in Proc. IEEE Int. Symp. on Inform. Theory (ISIT), (Adelaide, SA), September 2005.

[5] M. D. Yacoub, Foundations of Mobile Radio Engineering. New York: CRC Press, 1993.

[6] R. Rom and M. Sidi, Multiple Access Protocols. New York: Springer-Verlag, 1989.

[7] A. Krishna and R. LaMair, "A comparison of radio capture models and their effect on wireless LAN protocols," Proc. IEEE Int. Conf. on Universal Personal Commun. (ICUPC), pp. 666-672, 1994.

[8] L. G. Roberts, "Aloha packet system with and without slots and capture broadcast channels," ACM SIGCOMM Comp. Comm. Rev., vol. 5, p. 2842, April 1975.

[9] A. Busson, G. Chelius, and J. M. Gorce, "Interference modeling in CSMA multi-hop wireless networks," INRIA research report, No. 6624, 2009.

[10] F. Baccelli, B. Błaszczyszyn, and P. Muhlethaler, "A spatial reuse Aloha MAC protocol for multihop wireless mobile networks," in Proc. Allerton Conf. Commun., Control, Computing, (Monticello, IL), Oct. 2003.

[11] H. ElSawy and E. Hossain, "A modified hard core point process for analysis of random CSMA wireless networks in general fading environments," IEEE Trans. Commun., vol. 61, pp. 1520-1534, Apr. 2013.

[12] S. Stoyan, W. Kendall, and J. Mecke, Stochastic Geometry and Its Applications. Wiley, 1996.

[13] B. Matérn, Spatial Variation. Springer Lecture Notes in Statistics, 1986. 
[14] F. Baccelli, B. Błaszczyszyn, and P. Muhlethaler, "An Aloha protocol for multihop mobile wireless networks," IEEE Trans. Inform. Theory, vol. 52, pp. 421-436, Feb. 2006.

[15] J. Zhang and J. Andrews, "Distributed antenna systems with randomness," IEEE Trans. Wireless Comm., vol. 7, pp. 3636-3646, September 2008.

[16] R. K. Ganti and M. Haenggi, "Interference and outage in clustered wireless ad hoc networks," IEEE Trans. Inform. Theory, vol. 55, pp. 4067-4086, May 2009.

[17] C. Bordenave and G. L. Torrisi, "Large deviations of Poisson cluster processes," Stochastic Models, vol. 23, pp. 593-625, 2007.

[18] F. Baccelli and B. Błaszczyszyn, Stochastic Geometry and Wireless Networks - Volume I: Theory, vol. 3. Paris: Now, 2009.

[19] F. Baccelli and B. Błaszczyszyn, Stochastic Geometry and Wireless Networks - Volume II: Applications, vol. 4. Paris: Now, 2009.

[20] R. K. Ganti and M. Haenggi, Interference in Large Wireless Networks, vol. 3. Paris: Now, 2009.

[21] M. Haenggi, Stochastic Geometry for Wireless Networks. Cambridge University Press, 2012.

[22] P. Gupta and P. R. Kumar, "The capacity of wireless networks," IEEE Trans. Inform. Theory, vol. 46, pp. 388-404, Mar. 2000.

[23] A. Agarwal and P. R. Kumar, "Capacity bounds for ad hoc and hybrid wireless networks," ACM SIGCOMM Comp. Comm. Rev., vol. 34, pp. 71-81, April 2004.

[24] D. B. West, Introduction to Graph Theory. Prentice-Hall, 1996.

[25] I. Gronkvist and A. Hansson, "Comparison between graph-based and intereference based STDMA scheduling," in ACM International Symposium Mobile Ad Hoc Netwing and Computing (MOBIHOC), (Long Beach, CA), Oct. 2001.

[26] T. Moscibroda, R. Wattenhofer, and Y. Weber, "Protocol design beyond graph-based models," in ACM Symposium on Parallelism in algorithms and architectures, (New York, NY), Aug. 2006.

[27] H. ElSawy, E. Hossain, and M. Haenggi, "Stochastic geometry for modeling, analysis, and design of a multi-tier and cognitive cellular wireless network: A survey," IEEE Comm. Survey $\mathscr{E}$ Tuts., vol. 15, pp. 996-1015, Third Quarter 2013.

[28] M. Di Renzo and P. Guan, "Stochastic geometry modeling of coverage and rate of cellular networks using the Gil-Pelaez inversion theorem," IEEE Commun. Letters, vol. 18, pp. 1575-1578, Sept 2014. 
[29] S. Srinivasa and M. Haenggi, "Modeling interference in finite uniformly random networks," in Int. Workshop on Inform. Theory for Sensor Networks (WITS 2007), (Santa Fe, NM), Jun. 2007.

[30] X. Wang, Y. Huang, C. Cui, K. Chen, and M. Chen, "C-RAN: Evolution toward Green Radio Access Network," China Communications, vol. 7, pp. 107-112, 2010.

[31] P. Rost, C. Bernardos, A. D. Domenico, M. D. Girolamo, M. Lalam, A. Maeder, D. Sabella, and D. Wübben, "Cloud technologies for flexible 5G radio access networks," IEEE Commun. Magazine, vol. 52, May 2014.

[32] D. Wuebben, P. Rost, J. Bartelt, M. Lalam, V. Savin, M. Gorgoglione, A. Dekorsy, and G. Fettweis, "Benefits and impact of cloud computing on 5G signal processing," IEEE Signal Processing Magazine, vol. 31, pp. 35 - 44, Nov. 2014.

[33] M. Haenggi, J. Andrews, F. Baccelli, O.Dousse, and M. Franceschetti, "Stochastic geometry and random graphs for the analysis and design of wireless networks," IEEE J. Select. Areas Commun., vol. 27, pp. 1029-1046, Sept. 2009.

[34] M. Win, P. Pinto, and L. Shepp, "A mathematical theory of network interference and its applications," Proc. IEEE, vol. 97, pp. 205-230, Feb. 2009.

[35] J. G. Andrews, R. K. Ganti, M. Haenggi, N. Jindal, and S. Weber, "A primer on spatial modeling and analysis in wireless networks," IEEE Commun. Magazine, pp. 156-163, Nov. 2010.

[36] Z. Zhu, Q. Wang, Y. Lin, P. Gupta, A. Sarangi, S. Kalyanaraman, and H. Franke, "Virtual base station pool: Towards a wireless network cloud for radio access networks," in ACM International Conference on Computing Frontiers, (Ischia (Italy)), May 2011.

[37] C. W. M. Peng, V. Lau, and H. V. Poor, "Fronthaul-constrained cloud radio access networks: insights and challenges," vol. 22, Apr. 2015.

[38] R. K. Ganti and M. Haenggi, "Spatial and temporal correlation of the interference in ALOHA ad hoc networks," IEEE Commun. Letters, vol. 13, pp. 631-633, September 2009.

[39] M. Haenggi, "Diversity loss due to intereference correlation," IEEE Commun. Letters, vol. 16, pp. 1600-1603, October 2012.

[40] U. Schilcher, C. Bettstetter, and G. Brandner, "Temporal correlation of interference in wireless networks with Rayleigh block fading," vol. 11, pp. 2109-2120, December 2012.

[41] C. E. Koksal, "An analysis of the temporal correlation of interference in extended wireless networks," (Austin, Texas), December 2013.

[42] M. Haenggi, "The local delay in Poisson networks," IEEE Trans. Inform. Theory, vol. 59, pp. 1788-1802, March 2013. 
[43] Y. Zhong, W. Zhang, and M. Haenggi, "Managing interference correlation through random medium access," IEEE Trans. Wireless Comm., vol. 13, pp. 928-941, February 2014.

[44] Z. Gong and M. Haenggi, "Interference and outage in mobile random networks: Expectation, distribution, and correlation," vol. 13, pp. 337-349, February 2014.

[45] A. Hasan and J. G. Andrews, "The guard zone in wireless ad hoc networks," IEEE Trans. Wireless Comm., vol. 6, pp. 897-906, Mar. 2007.

[46] D. Torrieri and M. C. Valenti, "Exclusion and guard zones in DS-CDMA ad hoc networks," IEEE Trans. Commun., vol. 61, pp. 2468-2476, Jun. 2013.

[47] S. Talarico, M. C. Valenti, and D. Torrieri, "Analysis and optimization of a frequency hopping ad hoc network in Rayleigh fading," in Proc. Virginia Tech Symp. on Wireless Personal Commun., (Blacksburg, VA), Jun. 2012.

[48] Z. Khalid and S. Durrani, "Distance distributions in regular polygons," IEEE Trans. Veh. Tech., vol. 62, pp. 2363-2368, Jun. 2013.

[49] J. Guo, S. Durrani, and X. Zhou, "Outage probability in arbitrarily-shaped finite wireless networks," IEEE Trans. Commun., vol. 62, pp. 699-712, Feb. 2014.

[50] I. S. Gradshteyn and I. M. Ryzhik, Table of Integrals, Series, and Products. New York, NY: Academic Press, seventh ed., 1972.

[51] M. C. Valenti, D. Torrieri, and S. Talarico, "A direct approach to computing spatially averaged outage probability," IEEE Commun. Letters, vol. 18, pp. 1103-1106, July 2014.

[52] S. Weber, J. G. Andrews, and N. Jindal, "An overview of the transmission capacity of wireless networks," IEEE Trans. Commun., vol. 58, pp. 3593-3604, Dec. 2010.

[53] M. C. Valenti, D. Torrieri, and S. Talarico, "A new analysis of the DS-CDMA cellular downlink under spatial constraints," in Proc. IEEE Int. Conf. on Comp., Network. and Commun., (San Diego, CA), Jan. 2013.

[54] D. Torrieri, M. C. Valenti, and S. Talarico, "An analysis of the DS-CDMA cellular uplink for arbitrary and constrained topologies," IEEE Trans. Commun., vol. 61, pp. 3318-3326, Aug. 2013.

[55] M. C. Valenti, D. Torrieri, and S. Talarico, "A new analysis of the DS-CDMA cellular uplink under spatial constraints," in Proc. IEEE Int. Conf. on Commun. (ICC), (Budapest, Hungary), Jun. 2013.

[56] W. C. Y. Lee, "Elements of cellular mobile radio systems," IEEE Trans. Veh. Tech., vol. 35, pp. 48-56, May 1986.

[57] K. S. Gilhousen, I. M. Jacobs, R. Padovani, A. J. Viterbi, L. A. Weaver Jr., and C. E. Wheatley III, "On the capacity of a cellular CDMA system," IEEE Trans. Veh. Tech., vol. 40, pp. 303-312, May 1991. 
[58] A. J. Viterbi, CDMA Principles of Spread Spectrum Communication. Addison-Wesley, 1995.

[59] M. Zorzi, "On the analytical computation of the interference statistics with applications to the performance evaluation of mobile radio systems," IEEE Trans. Commun., vol. 45, pp. 103-109, Jan. 1997.

[60] T.-T. Vu, L. Decreusefond, and P. Martins, "An analytical model for evaluating outage and handover probability of cellular wireless networks," in IEEE Wireless Pers. Multimedia Comm. (WPMC), (Monticello, IL), Sept. 2012.

[61] J. Andrews, F. Baccelli, and R. K. Ganti, "A tractable approach to coverage and rate in cellular networks," IEEE Trans. Commun., vol. 59, pp. 3122-3134, Nov. 2011.

[62] H. S. Dhillon, R. K. Ganti, F. Baccelli, and J. G. Andrews, "Modeling and analysis of k-tier downlink heterogeneous cellular networks," IEEE J. Select. Areas Commun., vol. 30, Apr. 2012.

[63] H. S. Dhillon, T. D. Novlan, and J. G. Andrews, "Coverage probability of uplink cellular networks," in Proc. IEEE Global Telecommun. Conf. (GLOBECOM), (Anaheim, CA), Dec. 2012.

[64] B. Błaszczyszyn, M. K. Karray, and H. P. Keeler, "Using Poisson processes to model lattice cellular networks," in Joint Conference IEEE Computer and Communications Society, (Turin, Italy), Apr. 2013.

[65] T. D. Novlan, H. S. Dhillon, and J. G. Andrews, "Analytical modeling of uplink cellular networks," IEEE Trans. Wireless Comm., vol. 12, pp. 2669-2679, Jun. 2013.

[66] M. Di Renzo, A. Guidotti, and G. E. Corazza, "Average rate of downlink heterogeneous cellular networks over generalized fading channels: A stochastic geometry approach," IEEE Trans. Commun., vol. 61, pp. 3050-3071, May 2013.

[67] X. Zhang and J. G. Andrews, "Downlink cellular network analysis with multi-slope path loss models," IEEE Trans. Commun., vol. 63, p. 18811894, May 2015.

[68] T. S. Rappaport, S. Sun, R. Mayzus, H. Zhao, Y. Azar, K. Wang, G. N. Wong, J. K. Schulz, M. Samimi, and F. Gutierrez, "Millimeter wave mobile communications for $5 \mathrm{G}$ cellular: It will work!," IEEE Access, vol. 1, p. 335349, May 2013.

[69] S. Rangan, T. S. Rappaport, and E. Erkip, "Millimeter-wave cellular wireless networks: Potentials and challenges," Proc. IEEE, vol. 102, p. 366385, March 2014.

[70] L. Zhao and J. W. Mark, "Multi-step closed-loop power control using linear receivers for DS-CDMA systems," IEEE Trans. Wireless Comm., vol. 3, pp. 2141-2155, Nov. 2004.

[71] C. C. Chai, T. T. Tjhung, and L. C. Leck, "Combined power and rate adaptation for wireless cellular systems," IEEE Trans. Wireless Comm., vol. 4, pp. 6-13, Jan. 2005. 
[72] A. Subramanian and A. H. Sayed, "Joint rate and power control algorithms for wireless networks," IEEE Trans. Signal Proc., vol. 53, pp. 4204-4214, Nov. 2005.

[73] D. Torrieri, "Performance of direct-sequence systems with long pseudonoise sequences," IEEE J. Select. Areas Commun., vol. 10, pp. 770-781, May 1992.

[74] D. Torrieri, Principles of Spread-Spectrum Communication Systems, 3nd ed. New York: Springer, 2015.

[75] S. Weber, X. Yang, J. Andrews, and G. de Veciana, "Transmission capacity of wireless ad hoc networks with outage constraints," IEEE Trans. Inform. Theory, vol. 51, pp. 40914102, Dec. 2005.

[76] T. Eng, K. Ning, and L. B. Milstein, "Comparison of diversity combining techniques for Rayleigh-fading channels," IEEE Trans. Commun., vol. 44, pp. 1117-1129, Sep. 1999.

[77] P. Marsch and G. P. Fettweis, Coordinated Multi-Point in Mobile Communications: From Theory to Practice. Cambridge University Press, 2011.

[78] V. Aalo and C. Chayawan, "Outage probability of cellular radio systems using maximal ratio combining in rayleigh fading channel with multiple interferers," Electronics Letters, vol. 36, pp. 1314-1315, Jul. 2000.

[79] A. Shah and A. M. Haimovich, "Performance analysis of maximal ratio combining and comparison with optimum combining for mobile radio communications with cochannel interference," IEEE Trans. Veh. Tech., vol. 49, pp. 1454-1463, Jul. 2000.

[80] V. Aalo and J. Zhang, "Performance analysis of maximal ratio combining in the presence of multiple equal-power cochannel interferers in a Nakagami fading channel," IEEE Trans. Veh. Tech., vol. 50, pp. 497-503, Mar. 2001.

[81] J. Pena-Martin and J. Romero-Jerez, "Outage probability with MRC in presence of multiple interferers under Rayleigh fading channels," Electronics Letters, vol. 40, Sept. 2004.

[82] V. Aalo, T. Piboongungon, and G. P. Efthymoglou, "Another look at the performance of MRC schemes in nakagami-m fading channels with arbitrary parameters," IEEE Trans. Commun., vol. 53, pp. 2002-2005, Dec. 2005.

[83] W. Choi and J. G. Andrews, "Improved performance analysis for maximal ratio combining in asynchronous cdma channels," IEEE Trans. Wireless Comm., vol. 6, pp. 32973305, Sept. 2005.

[84] J. Romero-Jerez, J. Pena-Martin, and A. J. Goldsmith, "Outage probability of MRC with arbitrary power cochannel interferers in Nakagami fading," IEEE Trans. Commun., vol. 55, pp. 1283-1286, Jul. 2007.

[85] R. Tanbourgi, H. S. Dhillon, J. G. Andrews, and F. K. Jondral, "Effect of spatial interference correlation on the performance of maximum ratio combining," IEEE Trans. Wireless Comm., vol. 13, pp. 3307-3316, Jun. 2014. 
[86] R. Tanbourgi, H. S. Dhillon, J. G. Andrews, and F. K. Jondral, "Dual-branch MRC receivers under spatial interference correlation and Nakagami fading," IEEE Trans. Commun., vol. 62, pp. 1830 - 1844, Jun. 2014.

[87] S. Talarico, M. C. Valenti, and D. Torrieri, "Analysis of multi-cell downlink cooperation with a constrained spatial model," in Proc. IEEE Global Telecommun. Conf. (GLOBECOM), (Atlanta, GA), Dec. 2013.

[88] D. Gesbert, S. Hanly, H. Huang, S. Shitz, O. Simeone, and W. Yu, "Multi-cell MIMO cooperative networks: A new look at interference," IEEE J. Select. Areas Commun., vol. 28, pp. 1380-1408, Sep. 2010.

[89] O. Simeone, N. Levy, A. Sanderovich, O. Somekh, B. M. Zaidel, H. V. Poor, and S. Shitz, Cooperative Wireless Cellular Systems: An Information-Theoretic View. NOW: Foundations and Trends in Networking, 2011.

[90] R. Irmer, H. Droste, P. Marsch, M. Grieger, G. Fettweis, S. Brueck, H.-P. Mayer, L. Thiele, and V. Jungnickel, "Coordinated multipoint: Concepts, performance, and field trial results," IEEE Commun. Magazine, pp. 102-111, Feb. 2011.

[91] European Telecommunications Standards Institute, "LTE; Evolved universal terrestrial radio access network (E-UTRAN); X2 application protocol (X2AP)," 3GPP TS 136.423 version 11.4.0, Mar. 2013.

[92] A. Lozano, R. W. Heath, and J. G. Andrew, "Fundamental limits of cooperation," IEEE Trans. Inform. Theory, vol. 59, pp. 5213-5226, Sept. 2013.

[93] M. K. Karakayali, G. J. Foschini, and R. A. Valenzuela, "Network coordination for spectrally efficient communications in cellular systems," IEEE Trans. Wireless Comm., vol. 13, pp. 56-61, Apr. 2006.

[94] H. Dahrouj and W. Yu, "Coordinated beamforming for the multicell multi antenna wireless system," IEEE Trans. Wireless Comm., vol. 9, pp. 1748-1759, May 2010.

[95] European Telecommunications Standards Institute, "Universal mobile telecommunications system (UMTS); LTE; multimedia broadcast/multicast service (MBMS); protocols and codecs," 3GPP TS 126.346 version 11.4.0, Apr. 2013.

[96] A. Alexiou, C. Bouras, V. Kokkinos, A. Papazois, and G. Tsichritzis, "Spectral efficiency performance of MBSFN-enabled LTE networks," (Niagara Falls, Canada), Oct. 2010.

[97] L. Rong, O. Haddada, and S. Elayoubi, "Analytical analysis of the coverage of a MBSFN OFDMA network," in Proc. IEEE Global Telecommun. Conf. (GLOBECOM), (New Orleans, LA), Dec. 2008.

[98] A. Alexiou, C. Bouras, V. Kokkinos, and G. Tsichritzis, "Communication cost analysis of MBSFN in LTE," in Proc. IEEE Personal Indoor and Mobile Radio Commun. Conf., (Istanbul, Turkey), Sept. 2010. 
[99] A. Alexiou, C. Bouras, V. Kokkinos, and G. Tsichritzis, "Performance evaluation of LTE for MBSFN transmissions," Wireless Networks, vol. 18, pp. 227-240, Apr. 2012.

[100] S. Talarico and M. C. Valenti, "An accurate and efficient analysis of a MBSFN network," (Florence, Italy), May 2014.

[101] E. Dahlman, S. Parkvall, and J. Skold, 4 G LTE/LTE-Advanced for Mobile Broadband, Second Edition. Academic Press, 2013.

[102] M. Gudmundson, "Correlation model for shadow fading in mobile radio systems," Electronics Letters, vol. 27, pp. 2145 - 2146, Nov. 1991.

[103] European Telecommunications Standards Institute, "Universal mobile telecommunications system (UMTS); Selection procedures for the choice of radio transmission technologies of the UMTS (UMTS 30.03)," TR 101 112, Apr. 1998.

[104] A. Checko, H. L. Christiansen, Y. Yan, L. Scolari, G. Kardaras, M. S. Berger, and L. Dittmann, "Cloud RAN for mobile networks - a technology overview," IEEE Comm. Survey \& Tuts., vol. PP, pp. 1-23, Sep. 2014.

[105] S. Bhaumik, S. P. Chandrabose, M. K. Jataprolu, G. Kumar, A. Muralidhar, P. Polakos, V. Srinivasan, and T. Woo, "CloudIQ: A framework for processing base stations in a data center," in 18th Annual Inter. Conf. on Mobile Computing and Networking (MobiCom), (Istanbul, Turkey), Aug. 2012.

[106] Eurecom, "Overview - Open Air Interface," June 2014.

[107] European Telecommunications Standards Institute, "Radio access network; evolved universal terrestrial radio access; (E-UTRA); physical channels and modulation," tech. rep., 3GPP TS 35.211 version 10.6, Dec. 2012.

[108] European Telecommunications Standards Institute, "Radio access network; evolved universal terrestrial radio access; (E-UTRA); physical channels and modulation," tech. rep., 3GPP TS 35.211 version 11.1, Dec. 2012.

[109] P. Grover, K. A. Woyach, and A. Sahai, "Towards a communication-theoretic understanding of system-level power consumption," IEEE J. Select. Areas Commun., September 2011.

[110] M. C. Valenti, S. Talarico, and P. Rost, "The role of computational outage in dense cloud-based centralized radio access networks," in Proc. IEEE Global Telecommun. Conf. (GLOBECOM), (Austin, TX), Dec. 2014.

[111] P. Rost, S. Talarico, and M. C. Valenti, "The complexity-rate tradeoff of centralized radio access networks," IEEE Trans. Wireless Comm., 2015. to appear.

[112] M. Abramowitz and I. A. Stegun, Handbook of Mathematical Functions: with Formulas, Graphs, and Mathematical Tables. New York, NY: Dover Publications, 1965. 
[113] M. Coupechoux and J. M. Kelif, "How to set the fractional power control compensation factor in LTE?," in IEEE Sarnoff Symposium, (Princeton, NJ), May. 2011.

[114] M. C. Valenti and J. Sun, "The UMTS turbo code and an efficient decoder implementation suitable for software defined radios," Int. J. Wireless Info. Networks, vol. 8, pp. 203-216, Oct. 2001.

[115] W. Yu and J. M. Cioffi, "On constant power water-filling," in Proc. IEEE Int. Conf. on Commun. (ICC), (Helsinki, Finland), June 2001.

[116] P. Rost, A. Maeder, M. C. Valenti, and S. Talarico, "Computationally aware sumrate optimal scheduling for centralized radio access networks," in Proc. IEEE Global Telecommun. Conf. (GLOBECOM), (San Diego, CA), Dec. 2015.

[117] S. Weber, Y. Xiangying, G. de Veciana, and J. G. Andrews, "Transmission capacity of CDMA ad hoc networks," in Proc. IEEE Int. Symp. on Spread Spectrum Techniques and Applications (ISSSTA), (Sydney, Australia), Aug. 2004.

[118] J. Andrews, S. Weber, and M. Haenggi, "Ad hoc networks: To spread or not to spread?," IEEE Commun. Magazine, vol. 45, pp. 84-91, Dec. 2007.

[119] M. C. Valenti, D. Torrieri, and S. Talarico, "Adjacent channel interference in frequencyhopping ad hoc networks," in Proc. IEEE Int. Conf. on Commun. (ICC), (Budapest, Hungary), Jun. 2013.

[120] S. Cheng, R. I. Seshadri, M. Valenti, and D. Torrieri, "The capacity of noncoherent continuous-phase frequency shift keying," in Proc. Conf. on Information Sciences and Systems (CISS), (Baltimore, MD), Mar. 2007.

[121] D. Torrieri, S. Cheng, and M. Valenti, "Robust frequency hopping for interference and fading channels," IEEE Trans. Commun., vol. 56, pp. 1343-1351, Aug. 2008.

[122] J. G. Proakis and M. Salehi, Digital Communications. New York, NY: McGraw-Hill, Inc., fifth ed., 2008.

[123] S. Talarico, M. C. Valenti, and D. Torrieri, "Optimization of an adaptive frequencyhopping network," in Proc. IEEE Military Commun. Conf. (MILCOM), (Tampa, FL), Oct. 2015.

[124] M. C. Valenti, D. Torrieri, and S. Talarico, "Optimization of a finite frequency-hopping ad hoc network in Nakagami fading," in Proc. IEEE Military Commun. Conf. (MILCOM), (Orlando, FL), Oct. 2012.

[125] J. C. Lagarias, J. A. Reeds, M. H. Wright, and P. E. Wright, "Convergence properties of the Nelder-Mead simplex method in low dimensions.," SIAM J. Optim., vol. 9, no. 1, pp. 112-147, 1998.

[126] C. C. Wang and G. J. Pottie, "Variable bit allocation for FH-CDMA wireless communication systems," IEEE Trans. Commun., vol. 50, pp. 1637-1644, Dec. 2002. 
[127] Y. H. Lee and D. H. Kim, "Adaptive power and rate transmission in hybrid DS/FH CDMA communications over fading channels," vol. 7, Sept. 2013.

[128] J. G. Andrews, S. Weber, M. Kountouris, and M. Haenggi, "Random access transport capacity," IEEE Trans. Wireless Comm., vol. 9, p. 21012111, Jun. 2010.

[129] R. Vaze, "Throughput-delay-reliability tradeoff with ARQ in wireless ad hoc networks," IEEE Trans. Wireless Comm., vol. 10, p. 21422149, Jul. 2011.

[130] P. H. J. Nardelli and M. Latva-aho, "Efficiency of wireless networks under different hopping strategies," IEEE Trans. Wireless Comm., vol. 11, pp. 15-20, Jan. 2012.

[131] P. H. J. Nardelli, M. Kaynia, P. Cardieri, and M. Latva-aho, "Optimal transmission capacity of ad hoc networks with packet retransmissions," IEEE Trans. Wireless Comm., vol. 11, pp. 2760-2766, Aug. 2012.

[132] I. Byun, J. G. Andrews, and K. S. Kim, "Delay-constrained random access transport capacity," IEEE Trans. Wireless Comm., vol. 12, pp. 1628-1639, Apr. 2013.

[133] Y. Chen and J. G. Andrews, "An upper bound on multihop transmission capacity with dynamic routing selection," IEEE Trans. Inform. Theory, vol. 58, pp. 3751-3765, Jun. 2012 .

[134] A. Zanella, A. Bazzi, G. Pasolini, and B. M. Masini, "On the impact of routing strategies on the interference of ad hoc wireless networks," IEEE Trans. Commun., vol. 61, pp. 4322-4333, Oct. 2013.

[135] D. Torrieri, S. Talarico, and M. C. Valenti, "Multihop routing in ad hoc networks," in Proc. IEEE Military Commun. Conf. (MILCOM), (San Diego, CA), Nov. 2013.

[136] D. Torrieri, S. Talarico, and M. C. Valenti, "Performance analysis of geographic routing protocols in ad hoc networks," in Proc. IEEE Military Commun. Conf. (MILCOM), (Baltimore, MD), Oct. 2014.

[137] D. Torrieri, S. Talarico, and M. C. Valenti, "Performance comparisons of geographic routing protocols in mobile ad hoc networks," IEEE Trans. Commun., 2015. to appear.

[138] F. Cadger, K. Curran, J. Santos, and S. Moffett, "A survey of geographical routing in wireless ad hoc networks," IEEE Comm. Survey \& Tuts., vol. 15, pp. 621-653, 2nd quarter 2013.

[139] E. Perkins and M. Royer, "Ad hoc on demand distance vector routing," in Proc. IEEE Mobile Computing Systems and Applications (WMCSA), (New Orleans, LA), Feb. 1999.

[140] K. Z. Ghafoor, K. A. Bakar, J. Lloret, R. H. Khokhar, and K. C. Lee, "Intelligent beaconless geographical forwarding for urban vehicular environments," Wireless Networks, vol. 19, pp. 345-362, Apr. 2013. 
[141] S. Ruhrup and I. Stojmenovic, "Optimizing communication overhead while reducing path length in beaconless georouting with guaranteed delivery for wireless sensor networks," IEEE Trans. Computers, vol. 62, December 2013.

[142] C. Petrioli, M. Nati, P. Casari, M. Zorzi, and S. Basagni, "ALBA-R: Load-balancing geographic routing around connectivity holes in wireless sensor networks," IEEE Trans. Parallel and Distrib. Syst., vol. 25, pp. 529-539, March 2014.

[143] D. Torrieri and M. C. Valenti, "Exclusion and guard zones in DS-CDMA ad hoc networks," IEEE Trans. Commun., vol. 61, pp. 2468-2476, Jun. 2013.

[144] R. A. Brualdi, Introductory Combinatorics, 5th ed. Upper Saddle River,NJ: Pearson Prentice Hall, 2010.

[145] S. A. Hassan and M. A. Ingram, "A Quasi-Stationary Markov chain model of a cooperative multi-hop linear network," IEEE Trans. Wireless Comm., vol. 10, pp. 2306-2315, July 2011.

[146] N. Marchenko and C. Bettstetter, "Cooperative ARQ with relay selection: An analytical framework using semi-Markov processes," IEEE Trans. Veh. Tech., vol. 63, pp. 178190, Jan. 2014.

[147] H. Jung and M. A. Ingram, "Multi-packet opportunistic large array transmission on strip-shaped cooperative routes or networks," IEEE Trans. Wireless Comm., vol. 13, pp. 144-158, Jan. 2014.

[148] S. Talarico, M. C. Valenti, and T. R. Halford, "Unicast barrage relay networks: Outage analysis and optimization," in Proc. IEEE Military Commun. Conf. (MILCOM), (Austin, TX), Oct. 2014.

[149] C. M. Grinstead and J. Laurie, Introduction to Probability. AMS Bookstore, 1997.

[150] T. R. Halford and K. M. Chugg, "Barrage relay networks," in UCSD Inf. Theory and Appl. Workshop, (La Jolla, CA), Feb. 2010.

[151] T. R. Halford, T. A. Courtade, and K. A. Turck, "The user capacity of barrage relay networks," in Proc. IEEE Military Commun. Conf. (MILCOM), (Orlando, FL), Nov. 2012 .

[152] D. K. Lee and K. M. Chugg, "A pragmatic approach to cooperative communication," in Proc. IEEE Military Commun. Conf. (MILCOM), (Orlando, FL), Nov. 2006.

[153] T. R. Halford and K. M. Chugg, "The reliability multihop routes with autonomous cooperation," in UCSD Inf. Theory and Appl. Workshop, (La Jolla, CA), Feb. 2011.

[154] A. Chattopadhyay, A. Sinha, M. Coupechoux, and A. Kumar, "Optimal capacity relay node placement in a multi-hop network on a line," in IEEE Model. ES Opt. in Mob., Ad Hoc \& Wireless Networks (WiOpt), (Paderborn, DE), pp. 452-459, May 2012. 
[155] D. Torrieri and M. C. Valenti, "The outage probability of a finite ad hoc network in Nakagami fading," IEEE Trans. Commun., vol. 60, pp. 3509-3518, Nov. 2012.

[156] F. W. J. Olver, D. W. Lozier, R. F. Boisvert, and C. W. Clark, NIST Handbook of Mathematical Functions. Cambridge University Press, 2010. Available online at http://dlmf.nist.gov.

[157] S. Weber and J. G. Andrews, Transmission Capacity of Wireless Networks. Paris: Now, 2012.

[158] G. K. Karagiannidis, N. C. Sagias, and T. A. Tsiftsis, "Closed-form statistics for the sum of squared Nakagami-m variates and its applications," IEEE Trans. Commun., vol. 45, pp. 1353-1359, Aug. 2006.

[159] M. Geller and W. N. Edward, "A table of integrals of the exponential integral," Journal of Research of the National Bureau of Standards, vol. 71, p. 120, September 1969. 


\section{List of Publications}

[1] S. Talarico, M. C. Valenti, and D. Torrieri, "Analysis and optimization of a frequency hopping ad hoc network in Rayleigh fading," in Proc. Virginia Tech Symp. on Wireless Personal Commun., (Blacksburg, VA), Jun. 2012.

[2] M. C. Valenti, D. Torrieri, and S. Talarico, "A direct approach to computing spatially averaged outage probability," IEEE Commun. Letters, vol. 18, pp. 1103-1106, July. 2014.

[3] S. Talarico and M. C. Valenti, "Outage correlation in finite wireless networks," IEEE Wireless Comm. Letters, 2015. in preparation.

[4] M. C. Valenti, D. Torrieri, and S. Talarico, "A new analysis of the DS-CDMA cellular downlink under spatial constraints," in Proc. IEEE Int. Conf. on Comp., Network. and Commun., (San Diego, CA), Jan. 2013.

[5] D. Torrieri, M. C. Valenti, and S. Talarico, "A new analysis of the DS-CDMA cellular uplink under spatial constraints," in Proc. IEEE Int. Conf. on Commun. (ICC), (Budapest, Hungary), Jun. 2013.

[6] D. Torrieri, M. C. Valenti, and S. Talarico, "An analysis of the DS-CDMA cellular uplink for arbitrary and constrained topologies," IEEE Trans. Commun., vol. 61, pp. 3318-3326, Aug. 2013.

[7] S. Talarico, M. C. Valenti, and D. Torrieri, "Analysis of multi-cell downlink cooperation with a constrained spatial model," in Proc. IEEE Global Telecommun. Conf. (GLOBECOM), (Atlanta, GA), Dec. 2013.

[8] S. Talarico and M. C. Valenti, "An accurate and efficient analysis of a MBSFN network," in Proc. IEEE Int. Conf. on Acoust., Speech and Signal Processing (ICASSP), (Florence, Italy), May 2014.

[9] P. Rost, S. Talarico, and M. C. Valenti, "The complexity-rate tradeoff of centralized radio access networks," IEEE Trans. Wireless Comm., 2015. to appear.

[10] M. C. Valenti, S. Talarico, and P. Rost, "The role of computational outage in dense cloud-based centralized radio access networks," in Proc. IEEE Global Telecommun. Conf. (GLOBECOM), (Austin, TX), Dec. 2014. 
[11] P. Rost, A. Maeder, M. C. Valenti, and S. Talarico, "Computationally aware sumrate optimal scheduling for centralized radio access networks," in Proc. IEEE Global Telecommun. Conf. (GLOBECOM), (San Diego, CA), Dec. 2015.

[12] P. Rost, M. C. Valenti, S. Talarico, and A. Maeder, Cloud Radio Access Networks: Principles, Technologies, and Applications, ch. Computational Complexity and Achievable Rates in Centralized RAN. Cambridge: Cambridge University Press, 2015. to appear.

[13] M. C. Valenti, D. Torrieri, and S. Talarico, "Optimization of a finite frequency-hopping ad hoc network in Nakagami fading," in Proc. IEEE Military Commun. Conf. (MILCOM), (Orlando, FL), Oct. 2012.

[14] M. C. Valenti, D. Torrieri, and S. Talarico, "Adjacent channel interference in frequencyhopping ad hoc networks," in Proc. IEEE Int. Conf. on Commun. (ICC), (Budapest, Hungary), Jun. 2013.

[15] S. Talarico, M. C. Valenti, and D. Torrieri, "Optimization of an adaptive frequencyhopping network," in Proc. IEEE Military Commun. Conf. (MILCOM), (Tampa, FL), Oct. 2015.

[16] S. Talarico, M. C. Valenti, and D. Torrieri, "Analysis and optimization of a frequencyhopping ad hoc network," IEEE Trans. Commun., 2015. in preparation.

[17] D. Torrieri, S. Talarico, and M. C. Valenti, "Multihop routing in ad hoc networks," in Proc. IEEE Military Commun. Conf. (MILCOM), (San Diego, CA), Nov. 2013.

[18] D. Torrieri, S. Talarico, and M. C. Valenti, "Performance analysis of geographic routing protocols in ad hoc networks," in Proc. IEEE Military Commun. Conf. (MILCOM), (Baltimore, MD), Oct. 2014.

[19] D. Torrieri, S. Talarico, and M. C. Valenti, "Performance comparisons of geographic routing protocols in mobile ad hoc networks," IEEE Trans. Commun., 2015. to appear.

[20] S. Talarico, M. C. Valenti, and T. R. Halford, "Unicast barrage relay networks: Outage analysis and optimization," in Proc. IEEE Military Commun. Conf. (MILCOM), (Austin, TX), Oct. 2014.

[21] S. Talarico, M. C. Valenti, and T. R. Halford, "Stochastic modeling and analysis of unicast barrage relay networks," in IEEE Int. Conf. on Comp. Commun. (INFOCOM), 2016. submitted.

[22] D. Torrieri, S. Talarico, and M. C. Valenti, "Performance analysis of fifth-generation cellular uplink," in Proc. IEEE Military Commun. Conf. (MILCOM), (Tampa, FL), Oct. 2015.

[23] S. Talarico, N. A. Schmid, M. Alkhweldi, and M. C. Valenti, "Distributed estimation of a parametric field: Algorithms and performance analysis," IEEE Trans. Signal Proc., vol. 62, pp. 1041-1053, Mar. 2014. 